Online Supplementary Materials B. The testing dataset contains 643 Gramnegative bacterial proteins classified into 8 subcellular locations according to the experimental annotations. Both the accession numbers and sequences are given. None of the proteins has $\geq 25 \%$ sequence identity to any others in the same subcellular location of either the dataset here or the training dataset in the Online Supplementary Materials A. See the text of the paper for further explanation.

(1) 210 cytoplasm proteins

$>$ P27828

MKVLTVFGTRPEAIKMAPLVHALAKDPFFEAKVCVTAQHREMLDQVLKLFSIVPDYDLNI MQPGQGLTEITCRILEGLKPILAEFKPDVVLVHGDTTTTLATSLAAFYQRIPVGHVEAGL RTGDLYSPWPEEANRTLTGHLAMYHFSPTETSRQNLLRENVADSRIFITGNTVIDALLWV RDQVMSSDKLRSELAANYPF IDPDKKMILVTGHRRESFGRGFEEICHALADIATTHQDIQ IVYPVHLNPNVREPVNRILGHVKNVILIDPQEYLPFVWLMNHAWLILTDSGGIQEEAPSL GKPVLVMRDTTERPEAVTAGTVRLVGTDKQRIVEEVTRLLKDENEYQAMSRAHNPYGDGQ ACSRILEALKNNRISL

$>Q 02286$

MNPDNPLLALRDKISAVDKKLLTLLAERRLLAVEVAQAKLATHRPIRDVERERALLENLI VLGKAHNLDAHYITRLFQLVIEDSVLTQQALLQKNLNHPHAHAARIAFLGPKGSYSHLAA RNYASRHFDSMVECGCLKFHDI I KQVENGVADYAVMP IENTSSGS INDVYDLLQQTSLS I VGELTLPIDHCVLVNGPTDLQQIETVYSHPQPFQQCSQFINRFPHWKIEYTESTAAAMEK VAALNSPKVAALGSEAGGELYQLQVLERNLANQQQNHTRF IVLARKAIEVSDQVPAKTTL IMATGQQAGALVDALLVLRQHNLIMSKLESRP INGNPWEEMFY IDVQGNLQSERMQQALQ ELQTMTRSLKVLGCYPSENVVPAEPGR

$>085094$

MSTEDLYQEDVEMLDDYEDPSTEQHWSEEDGEPSGYATAEPDDHAAQEEQDEPPALDSLA LDLTLRCGELRLTLAELRRLDAGTILEVTGISPGHATLCHGEQVVAEGELVDVEGRLGLQ ITRLVTRS

$>$ P0A 890

MTDLFSSPDHTLDALGLRCPEPVMMVRKTVRNMQPGETLLI IADDPATTRDI PGFCTFME HELVAKETDGLPYRYLIRKGG

$>$ P29930

SDETTVGGEAPAEKDDARHAMKMAKKKAAREKIMATKTDEKGLI IVNTGKGKGKSTAGFG MI FRHIAHGMPCAVVQF I KGAMATGERELIEKHFGDVCQFYTLGEGFTWETQDRARDVAM AEKAWEKAKELIRDERNSMVLLDEINIALRYDYIDVAEVVRFLKEEKPHMTHVVLTGRNA KEDLIEVADLVTEMELIKHPFRSGIKAQQGVEF

$>$ P08308

AFNMHNRNLLSLMHHSTRELRYLLDLSRDLKRAKYTGTEQQHLKRKNIALIFEKTSTRTR CAFEVAAYDQGANVTYIDPNSSQIGHKESMKDTARVLGRMYDAIEYRGFKQEIVEELAKF AGVPVFNGLTDEYHPTQMLADVLTMREHSDKPLHDISYAYLGDARNNMGNSLLLIGAKLG MDVRIAAPKALWPHDEFVAQCKKFAEESGAKLTLTEDPKEAVKGVDFVHTDVWVSMGEPV EAWGERI KELLPYQVNMEIMKATGNPRAKFMHCLPAFHNSETKVGKQIAEQYPNLANGIE VTEDVFESPYNIAFEQAENRMHTIKAILVSTLADI

$>$ P23619

MKIVSDAKHTGRTRCTIHCQNCS ISQLCLPFTLSEHELTQLDNI IERKKPVQKSQI IFQS GDELRS IYAIRSGTIKSYTISESGEEQITAFHLPGDLVGFDAIMNMKHVGFAQALETSMI CEI PFDILDDLAGKMPKIRHQIMRLMSNEI KSDQEMI LLLSKMSAEEKLAAFLHNLSQRY AAPGFSAREFRLTMTRGDIGNYLGLTIETISRLLGRFQKSGMITVQGKYITINRMDELTV $>$ P0A968

MEKGTVKWFNNAKGFGF ICPEGGGEDIFAHYSTIQMDGYRTLKAGQSVQFDVHQGPKGNH ASVIVPVEVEAAVA

$>$ P46849

MKRMIALDGAQGEGGGQILRSALSLSMITGQPFTITSIRAGRAKPGLLRQHLTAVKAATE ICGATVEGAELGSQRLLFRPGTVRGGDYRFAIGSAGSCTLVLQTVLPALWFADGPSRVEV SGGTDNPSAPPADFIRRVLEPLLAKIGIHQQTTLLRHGFYPAGGGVVATEVSPVASFNTL QLGERGNIVQMRGEVLLAGVPRHVAEREIATLAGSFSLHEQNIHNLPRDQGPGNTVSLEV 
ESENITERFFVVGEKRVSAEVVAAQLVKEVKRYLASTAAVGEYLADQLVLPMALAGAGEF TVAHPSCHLLTNIAVVERFLPVRFSLIETDGVTRVSIE

$>$ P07294

MAKVQAYVSDEIVYKINKIVERRRAEGAKSTDVSFSSISTMLLELGLRVYEAQMERKESA FNQAEFNKVLLECAVKTQSTVAKILGIESLSPHVSGNPKFEYANMVEDIRDKVSSEMERF FPENDEE

$>$ Q5HTT3

MAEITAAMVKELRESTGAGMMDCKNALSETNGDFDKAVQLLREKGLGKAAKKADRLAAEG LVSVKVSDDFTSATVSEINSETDFVAKNDQFIALTKDTTAHIQSNSLQSVEELHSSTING VKFEEYLKSQIATIGENLVVRRFATLKAGANGVVNGY I HTNGRVGVVIAAACDSTEVASK SRDLLRQICMHIAAMRPSYLSYEDLDMTFVENEYKALVAELEKENEERRRLKDPNKPEHK I PQFASRKQLSDA I LKEAEEKI KEELKAQGKPEKIWDNI I PGKMNSF IADNSQLDSKLTL MGQFYVLDDKKTVEQVIAEKEKEFGGKI KIVEFICFEVGEGLEKKTEDFAAEVAAQL

$>$ P10371

MI I PALDLIDGTVVRLHQGDYGKQRDYGNDPLPRLQDYAAQGAEVLHLVDLTGAKDPAKR QI PLIKTLVAGVNVPVQVGGGVRSEEDVAALLEAGVARVVVGSTAVKSQDMVKGWFERFG ADALVLALDVRIDEQGNKQVAVSGWQENSGVSLEQLVETYLPVGLKHVLCTDISRDGTLA GSNVSLYEEVCARYPQVAFQSSGGIGDIDDVAALRGTGVRGVIVGRALLEGKFTVKEAIA CWQNA

$>$ P07004

MLEQMGIAAKQASYKLAQLSSREKNRVLEKIADELEAQSEI I LNANAQDVADARANGLSE AMLDRLALTPARLKGIADDVRQVCNLADPVGQVIDGGVLDSGLRLERRRVPLGVIGVIYE ARPNVTVDVASLCLKTGNAVILRGGKETCRTNAATVAVIQDALKSCGLPAGAVQAIDNPD RALVSEMLRMDKYIDMLI PRGGAGLHKLCREQSTI PVITGGIGVCHIYVDESVEIAEALK VIVNAKTQRPSTCNTVETLLVNKNIADSFLPALSKQMAESGVTLHADAAALAQLQAGPAK VVAVKAEEYDDEFLSLDLNVKIVSDLDDAIAHIREHGTQHSDAILTRDMRNAQRFVNEVD SSAVYVNASTRFTDGGQFGLGAEVAVSTQKLHARGPMGLEALTTYKWIGIGDYTIRA

$>$ P30335

MPITDLVAPEAILPALKVNSKKQALQELAAKAAELTGQNERAVFEVLLQREKLGTTAVGY GVAI PHGKLPKLEKI FGLFARLDRP IDFESMDGQPVDLVFLLLAPEGAGADHLKALARIA RLLRDQDIAKKLRASRDAQAIYSVLALPPA

$>$ P22319

MRAPHKDEIASHELPATPMDPALAANREGKIKVATIGLCGCWGCTLSFLDMDERLLPLLE KVTLLRSSLTDIKRI PERCAIGFVEGGVSSEENIETLEHFRENCDILISVGACAVWGGVP AMRNVFELKDCLAEAYVNSATAVPGAKAVVPFHPDI PRITTKVYPCHEVVKMDYF I PGCP PDGDAIFKVLDDLVNGRPFDLPSSINRYD

$>$ P21517

MMLNAWHLPVPPFVKQSKDQLLITLWLTGEDPPQRIMLRTEHDNEEMSVPMHKQRSQPQP GVTAWRAAIDLSSGQPRRRYSFKLLWHDRQRWFTPQGFSRMPPARLEQFAVDVPDIGPQW AADQ I FYQI FPDRFARSLPREAEQDHVYYHHAAGQEI I LRDWDEPVTAQAGGSTFYGGDL DGISEKLPYLKKLGVTALYLNPVFKAPSVHKYDTEDYRHVDPQFGGDGALLRLRHNTQQL GMRLVLDGVFNHSGDSHAWFDRHNRGTGGACHNPESPWRDWYSFSDDGTALDWLGYASLP KLDYQSESLVNEIYRGEDS IVRHWLKAPWNMD GWRLDVVHMLGEAGGARNNMOHVAGITE AAKETQPEAYIVGEHFGDARQWLQADVEDAAMNYRGFTFPLWGFLANTDISYDPQQIDAQ TCMAWMDNYRAGLSHQQQLRMFNQLDSHDTARFKTLLGRDIARLPLAVVWLFTWPGVPCI YYGDEVGLDGKNDPFCRKPFPWQVEKQDTALFALYQRMIALRKKSQALRHGGCQVLYAED NVVVFVRVLNQQRVLVAINRGEACEVVLPASPFLNAVQWQCKEGHGQLTDGILALPAISA TVWMN

$>$ P94212

MTDRSTLDDAPAQADF I IAGATLIDGGGGPARQGDLAVRGGRIVALGDFAHAPGVPVIDA RGLALAPGFIDSHTHDDGYLLAHPEMLPKVSQGITTVVTGNCGISLAPLSRRQIPQPLDL LGPPELFRFATFRDWLRALAETPAAVNVI PLVGHTTLRVAVMDDTGRAATDAERAAMRAL LDEALQAGAFGVSTGTFYPPASAAPTDEI IDVCQPLRGRAGAIYATHLRDEADHIVPAME EALLIGRELDCRVVFSHHKLAGERNHGRSRETLDMISRAAATQRVCLDCHPYPATSTMLR LDRARLASRTLITWSKGYPEATGRDFSEVMAELGLDDEAAIARLAPAGAIYFLMDQADVN RIFSHPLTTVGSDGLPFDPHPHPRQWGTFTNVLRTMVREQRLLSLETAIHKMTGLAAAQY GLTERGLLRQGYHADLVLFDPANVTDTATFSAPIQVSQGIHAVWVNGRQVWDGERTGAER PGQVLAPGDAIPWSQQSE

$>$ P25080 
TDNNKYRDVEIRAPRGNKLTAKSWLTEAPLRMLMNNLDPQVAENPKELVVYGGIGRAARN WECYDKIVETLTRLEDDETLLVQSGKPVGVFKTHSNAPRVLIANSNLVPHWANWEHFNEL DAKGLAMYGQMTAGSWIYIGSQGIVQGTYETFVEAGRQHYGGTVKAKWVLTAGLGGMGGA QPLAATLAGACSLNIECQQSRIDFRLETRYVDEQATDLDDALVRIAKYTAEGKAISIALH GNAAEILPELVKRGVRPDMVTDQTSAHDPLNGYLPAGWTWEQYRDRAQTEPAAVVKAAKQ SMAVHVQAMLDFQKQGVPTFDYGNNIRQMAKEEGVANAFDFPGFVPAYIRPLFCRGVGPF RWAALSGEAEDIYKTDAKVKELI PDDAHLHRWLDMARER I SFQGLPAR I CWVGLGLRAKL GLAFNEMVRSGELSAPVVIGRDHLDSGSVSSPNRETEAMRDGSDAVSDWPLLNALLNTAG GATWVSLHHGGGVGMGFSQHSGMVIVCDGTDEAAERIARVLTNDPGTGVMRHADAGYDIA IDCAKEQGLDLPMITG

$>$ POA9F6

MSKRLPPLNALRVFDAAARHLSFTRAAEELFVTQAAVSHQIKSLEDFLGLKLFRRRNRSL LLTEEGQSYFLDIKEIFSQLTEATRKLQARSAKGALTVSLLPSFAIHWLVPRLSSFNSAY PGIDVRIQAVDRQEDKLADDVDVAIFYGRGNWPGLRVEKLYAEYLLPVCSPLLLTGEKPL KTPEDLAKHTLLHDASRRDWQTYTRQLGLNH INVQQGP IFSHSAMVLQAAIHGQGVALAN NVMAQSEIEAGRLVCPFNDVLVSKNAFYLVCHDSQAELGKIAAFRQWILAKAAAEQEKFR FRYEQ

$>$ P26602

SHPALTQLRALRYCKEI PALDPQLLDWLLLEDSMTKRFEQQGKTVSVTMIREGFVEQNEI PEELPLLPKESRYWLREILLCADGEPWLAGRTVVPVSTLSGPELALQKLGKTPLGRYLFT SSTLTRDFIEIGRDAGLWGRRSRLRLSGKPLLLTELFLPASPLY

$>$ Q 87AY6

MSSYLFTSESVSEGHPDKVADQISDAVLDAILVQDPRARVACETMVKTGVAI IAGEITTS AWVDLEALTRKVIVDIGYNSSDVGFDGATCGVLNLIGKQSPDINQGVDRKKAEEQGAGDQ GLMFGYATNETDSYMPAAIHISHRLVEQQAKIRKKSNSLLPWLRPDAKAQITLRYEDGVA SAIDAVVLSTQHDPGIKQKDLVEAVREEILKPVLPSKWLHKGTKFHINPTGKFVIGGPVG DCGLTGRKI IVDTYGGSARHGGGAF SGKDPSKVDRSASYAVRYVAKNVVAAGLADRCEVQ VSYAIGVAEPISISVTTFGTGKI PDDKIEKLI CQHFDLRPYGI I KMLDLIHPIYQPSASY GHFGRKPRELAYTNSNGERVVATAFSWEKIDKAEVLRADA

$>Q 46 \mathrm{M} 54$

MEFRQLRYFVAAAEEGNVGAAARRLHISQPPVTRQIHALEQHLGVLLFERSARGVQLTPA GAAFLEDARRMLELGRTSVDRSRAASRGEIGQLDIGYLGTAIYQTVPALLHAFTQAVPGA TLSLALMPKVRQIEALRAGT IHLGVGRFYPQEPGITVEHLHYERLYIAAGSS IARQLRQD PTLLRLKSESLVLFPKEGRPSFADEVIALMRRAGVEPRVTAIVEDVNAALGLVAAGAGVT LVPASVAAIRRPFVRTMEMADASAKVPVSLTYLTDSRVPVLRAFLDVARRGKGQK

$>$ P62601

MLNQKIQNPNPDELMIEVDLCYELDPYELKLDEMIEAEPEPEMIEGLPASDALTPADRYL ELFEHVQSAKIFPDSKTFPDCAPKMDPLDILIRYRKVRRHRDFDLRKFVENHFWLPEVYS SEYVSDPQNSLKEHIDQLWPVLTREPQDHI PWSSLLALPQSYIVPGGRFSETYYWDSYFT MLGLAESGREDLLKCMADNFAWMIENYGHI PNGNRTYYLSRSQPPVFALMVELFEEDGVR GARRYLDHLKMEYAFWMDGAESLI PNQAYRHVVRMPDGSLLNRYWDDRDTPRDESWLEDV ETAKHSGRPPNEVYRDLRAGAASGWDYSSRWLRDTGRLAS IRTTQF I PIDLNAFLFKLES AIANISALKGEKETEALFRQKASARRDAVNRYLWDDENGIYRDYDWRREQLALFSAAAIV PLYVGMANHEQADRLANAVRSRLLTPGGILASEYETGEQWDKPNGWAPLQWMAIQGFKMY GDDLLGDEIARSWLKTVNQFYLEQHKLIEKYHIADGVPREGGGGEYPLQDGFGWTNGVVR RLIGLYGEP

$>$ P04036

MHDANIRVAIAGAGGRMGRQLIQAALALEGVQLGAALEREGSSLLGSDAGELAGAGKTGV TVQSSLDAVKDDFDVF IDFTRPEGTLNHLAFCRQHGKGMVIGTTGFDEAGKQAIRDAAAD IAIVFAANFSVGVNVMLKLLEKAAKVMGDYTDIEI IEAHHRHKVDAPSGTALAMGEAIAH ALDKDLKDCAVYSREGHTGERVPGTIGFATVRAGDIVGEHTAMFADIGERLEITHKASSR MTFANGAVRSALWLSGKESGLFDMRDVLDLNNL

$>$ P12994

MKLISNDLRDGDKLPHRHVFNGMGYDGDNISPHLAWDDVPAGTKSFVVTCYDPDAPTGSG WWHWVVVNLPADTRVLPQGFGSGLVAMPDGVLQTRTDFGKTGYDGAAPPKGETHRYIFTV HALDIER IDVDEGASGAMVGFNVHFHSLASAS ITAMFS

$>$ P 76008

MSLFHLIAPSGYCIKQHAALRGIQRLTDAGHQVNNVEVIARRCERFAGTETERLEDLNSL ARLTTPNTIVLAVRGGYGASRLLADIDWQALVARQQHDPLLI CGHSDFTAIQCGLLAHGN 
VITFSGPMLVANFGADELNAFTEHHFWLALRNETFTIEWQGEGPTCRAEGTLWGGNLAML ISLIGTPWMPKIENGILVLEDINEHPFRVERMLLQLYHAGILPRQKAI ILGSFSGSTPND YDAGYNLESVYAFLRSRLS I PLITGLDFGHEQRTVTLPLGAHA I LNNTREGTQLTISGHP $>$ P15034

SEISRQEFQRRRQALVEQMQPGSAALIFAAPEVTRSADSEYPYRQNSDFWYFTGFNEPEA VLVLIKSDDTHNHSVLFNRVRDLTAEIWFGRRLGQDAAPEKLGVDRALAF SEINQQLYQL LNGLDVVYHAQGEYAYADVIVNSALEKLRKGSRQNLTAPATMIDWRPVVHEMRLFKSPEE IAVLRRAGEITAMAHTRAMEKCRPGMFEYHLEGEIHHEFNRHGARYPSYNTIVGSGENGC ILHYTENECEMRDGDLVLIDAGCEYKGYAGDITRTFPVNGKFTQAQREIYDIVLESLETS LRLYRPGTS ILEVTGEVVRIMVSGLVKLGILKGDVDELIAQNAHRP F FMHGLSHWLGLDV HDVGVYGQDRSRILEPGMVLTVEPGLYIAPDAEVPEQYRGIGIRIEDDIVITETGNENLT ASVVKKPEEIEALMVAARKQ

$>$ P22259

MRVNNGLTPQELEAYGISDVHDIVYNPSYDLLYQEELDPSLTGYERGVLTNLGAVAVDTG IFTGRSPKDKYIVRDDTTRDTFWWADKGKGKNDNKPLSPETWQHLKGLVTRQLSGKRLFV VDAFCGANPDTRLSVRF ITEVAWQAHFVKNMF IRP SDEELAGFKPDF IVMNGAKCTNPQW KEQGLNSENFVAFNLTERMQLIGGTWYGGEMKKGMFSMMNYLLPLKGIASMHCSANVGEK GDVAVFFGLSGTGKTTLSTDPKRRLIGDDEHGWDDDGVFNFEGGCYAKTIKLSKEAEPEI YNAIRRDALLENVTVREDGTIDFDDGSKTENTRVSYPIYHIDNIVKPVSKAGHATKVIFL TADAFGVLPPVSRLTADQTQYHFLSGFTAKLAGTERGITEPTPTFSACFGAAFLSLHPTQ YAEVLVKRMQAAGAQAYLVNTGWNGTGKR I S I KDTRA I IDA I LNGSLDNAETFTLPMFNL AIPTELPGVDTKILDPRNTYASPEQWQEKAETLAKLF IDNFDKYTDTPAGAALVAAGPKL $>$ P06149

SSMTTTDNKAFLNELARLVGSSHLLTDPAKTARYRKGFRSGQGDALAVVF PGSLLELWRV LKACVTADKI I LMQAANTGLTEGSTPNGNDYDRDVVI ISTLRLDKLHVLGKGEQVLAYPG TTLYSLEKALKPLGREPHSVIGSSCIGASVIGGI CNNSGGSLVQRGPAYTEMSLFARINE DGKLTLVNHLGIDLGETPEQILSKLDDDR I KDDDVRHDGRHAHDYDYVHRVRDIEADTPA RYNADPDRLFESSGCAGKLAVFAVRLDTFEAEKNQQVFYIGTNQPEVLTEIRRHILANFE NLPVAGEYMHRDIYDIAEKYGKDTFLMIDKLGTDKMPFFFNLKGRTDAMLEKVKFFRPHF TDRAMQKFGHLFP SHLP PRMKNWRDKYEHHLLLKMAGDGVGEAKSWLVDYFKQAEGDFFV CTPEEGSKAFLHRFAAAGAAIRYQAVHSDEVEDI LALDIALRRNDTEWYEHLPPEIDSQL VHKLYYGHFMCYVFHQDYIVKKGVDVHALKEQMLELLQQRGAQYPAEHNVGHLYKAPETL QKFYRENDPTNSMNPGIGKTSKRKNWQEVE

$>006644$

TKPLDGINVLDFTHVQAGPACTQMMGFLGANVIKIERRGSGDMTRGWLQDKPNVDSLYFT MFNCNKRS IELDMKTPEGKELLEQMIKKADVMVENFGPGALDRMGFTWEYIQELNPRVIL ASVKGYAEGHANEHLKVYENVAQCSGGAAATTGFWDGPPTVSGAALGDSNSGMHLMIGIL AALEMRHKTGRGQKVAVAMQDAVLNLVRIKLRDQQRLERTGILAEYPQAQPNFAFDRDGN PLSFDNITSVPRGGNAGGGGQPGWMLKCKGWETDADSYVYFTIAANMWPQICDMIDKPEW KDDPAYNTFEGRVDKLMDIFSFIETKFADKDKFEVTEWAAQYGI PCGPVMSMKELAHDPS LQKVGTVVEVVDEIRGNHLTVGAPFKFSGFQPEITRAPLLGEHTDEVLKELGLDDAKIKE LHAKQVV

$>$ P13000

SKRYFVTGTDTEVGKTVASCALLQAAKAAGYRTAGYKPVASGSEKTPEGLRNSDALALQR NSSLQLDYATVNPYTFAEPTSPHI ISAQEGRP IESLVMSAGLRALEQQADWVLVEGAGGW FTPLSDTFTFADWVTQEQLPVILVVGVKLGCINHAMLTAQVIQHAGLTLAGWVANDVTPP GKRHAEYMTTLTRMIPAPLLGEI PWLAENPENAATGKYINLALL

$>$ P30850

MFQDNPLLAQLKQQLHSQTPRAEGVVKATEKGFGFLEVDAQKSYF I P PPQMKKVMHGDR I IAVIHSEKERESAEPEELVEPFLTRFVGKVQGKNDRLAIVPDHPLLKDAIPCRAARGLNH EFKEGDWAVAEMRRHPLKGDRSFYAELTQYITFGDDHFVPWWVTLARHNLEKEAPDGVAT EMLDEGLVREDLTALDFVTIDSASTEDMDDALFAKALPDDKLQLIVAIADPTAWIAEGSK LDKAAKIRAFTNYLPGFNI PMLPRELSDDLCSLRANEVRPVLACRMTLSADGTIEDNIEF FAATIESKAKLVYDQVSDWLENTGDWQPESEA IAEQVRLLAQI CQRRGEWRHNHALVFKD RPDYRF ILGEKGEVLDIVAEPRR IANR IVEEAMIAAN I CAARVLRDKLGFGIYNVHMGFD PANADALAALLKTHGLHVDAEEVLTLDGFCKLRRELDAQPTGFLDSRIRRFQSFAEISTE PGPHFGLGLEAYATWTSPIRKYGDMINHRLLKAVIKGETATRPQDEITVQMAERRRLNRM AERDVGDWLYARFLKDKAGTDTRFAAEIVDISRGGMRVRLVDNGAIAF I PAPFLHAVRDE LVCSQENGTVQIKGETVYKVTDVIDVTIAEVRMETRS I IARPVA 
$>$ P06722

SQPRPLLSPPETEEQLLAQAQQLSGYTLGELAALVGLVTPENLKRDKGWIGVLLEIWLGA SAGSKPEQDFAALGVELKTI PVDSLGRPLETTFVCVAPLTGNSGVTWETSHVRHKLKRVL WI PVEGERS I PLAQRRVGS PLLWS PNEEEDRQLREDWEELMDMIVLGQVERITARHGEYL QIRPKAANAKALTEAIGARGERILTLPRGFYLKKNFTSALLARHFLIQ

$>$ P0A9G6

MKTRTQQIEELQKEWTQPRWEGITRPYSAEDVVKLRGSVNPECTLAQLGAAKMWRLLHGE SKKGYINSLGALTGGQALQQAKAGIEAVYLSGWQVAADANLAASMYPDQSLYPANSVPAV VERINNTFRRADQIQWSAGIEPGDPRYVDYFLPIVADAEAGFGGVLNAFELMKAMIEAGA AAVHFEDQLASVKKCGHMGGKVLVPTQEA IQKLVAARLAADVTGVPTLLVARTDADAADL ITSDCDPYDSEFITGERTSEGFFRTHAGIEQAISRGLAYAPYADLVWCETSTPDLELARR FAQAIHAKYPGKLLAYNCSP SFNWQKNLDDKTIASFQQQLSDMGYKFQF ITLAGIHSMWF NMFDLANAYAQGEGMKHYVEKVQQPEFAAAKDGYTFVSHQQEVGTGYFDKVTTI IQGGTS SVTALTGSTEESQF

$>$ P0A9G8

MQAEILLTLKLQQKLFADPRRISLLKHIALSGS ISQGAKDAGISYKSAWDAINEMNQLSE HILVERATGGKGGGGAVLTRYGQRLIQLYDLLAQIQQKAFDVLSDDDALPLNSLLAAISR FSLQTSARNQWFGTITARDHDDVQQHVDVLLADGKTRLKVAITAQSGARLGLDEGKEVLI LLKAPWVGITQDEAVAQNADNQLPGI ISH IERGAEQCEVLMALPDGQTLCATVPVNEATS LQQGQNVTAYFNADSVIIATLC

$>$ P31658

TVQTSKNPQVDIAEDNAFFPSEYSLSQYTSPVSDLDGVDYPKPYRGKHKILVIAADERYL PTDNGKLFSTGNHP IETLLPLYHLHAAGFEFEVATISGLMTKFEYWAMPHKDEKVMPFFE QHKSLFRNPKKLADVVASLNADSEYAAIFVPGGHGALIGLPESQDVAAALQWAI KNDRFV ISLCHGPAAFLALRHGDNPLNGYS I CAFPDAADKQTPEIGYMPGHLTWYFGEELKKMGMN I INDDITGRVHKDRKLLTGDSPFAANALGKLAAQEMLAAYAG

$>$ POA988

MKFTVEREHLLKPLQQVSGPLGGRPTLPILGNLLLQVADGTLSLTGTDLEMEMVARVALV QPHEPGATTVPARKFFDICRGLPEGAEIAVQLEGERMLVRSGRSRFSLSTLPAADFPNLD DWQSEVEFTLPQATMKRLIEATQFSMAHQDVRYYLNGMLFETEGEELRTVATDGHRLAVC SMPIGQSLPSHSVIVPRKGVIELMRMLDGGDNPLRVQIGSNNIRAHVGDFIFTSKLVDGR FPDYRRVLPKNPDKHLEAGCDLLKQAFARAAILSNEKFRGVRLYVSENQLKITANNPEQE EAEEILDVTYSGAEMEIGFNVSYVLDVLNALKCENVRMMLTDSVSSVQIEDAASQSAAYV VMPMRL

$>$ Q09064

MI IERLVGNLRDLNPLDFSVDHVDLEWFETRKKIARFKTRQGKDIAIRLKDAPKLGLSQG DILFKEEKEI IAVNILDSEVIHIQAKSVAEVAKICYEIGNRHAALYYGESQFEFKTPFEK PTLALLEKLGVQNRVLSSKLDSKERLTVSMPHSEPNFKVSLASDFKVVVK

$>$ P56124

MLFSKLFAPTLKEP PKDAVLKSHKHLAQAGYIYQVGSGIYNFLPLAKKVLDKIENITHKR MQEHGAQNILMSFVVLASLWEKSGRLDKYGKELLVFKDRKDNDFVLSPTLEENITEIAAN FIKSYKQLPVHLYQIHTKFRDEIRPRFGLVRAREF IMKDGYSFHEDAESLDKEFLNTQSA YKEILSDLGLDFRIVEADSGAIGGSKSREFVVLTECGEDTIVVCQNCDYAANIEIAKRSK RPEPLNVPKAQLAKFPTPNTTSAQSVAEFFKTEPYFVLKALVRKVIHKDKETLACFFVRG DDNLEEVKALNALN I I GANALELREASQKDLDNVGLIAGF I GPYGLKKHVSY I I FDEDLK EGDCLIAGANEKDFHAVGVDLKGFENLVYADIVQVKESDRCPNCQGALKYHKSLEVGHIF KLGQGYAKSLKASFLDKNGKEQFFEMGCYGIGISRLLSAILEQKSDDLGCVWTKNTAPFD VVIVVSNWKDEAQKKLAFEVYERLLQKGVDALLDDRDARFGAKMRDFELIGERLALI IGK QTLESKEFECIKRANLEKQTIKDIELEEKILEMLESE

$>Q 51480$

MNLRPLAPLLLTLLAGCSQQPPLRGSGDLGVLIERADGSVQILDGTAKTSLARVEGLGDL SHASLVFSRDQRYAYVFGRDGGLTKLDLLAQR IDKRLIQGGNS I GGA I SQDGRLVAVSNY EPGGVKVFDSRTLELVAEIPATRLPGQDRNSRVVGLVDAPGQRFVFSLFDSGEIWIADFS QGDTPHLTRFRDIGKQPYDALISPDGRYYMAGLFGEDGMAQLDLWHPERGVRRVLGDYGR GQRKLPVYKMPHLEGWTIASDQAFVPAVGHHQVLVLDARDWKQTDA IDVAGQPVFVMTRP DDRQIWVNFAYPDNDKVQVIDSETHEVIETLRPGPGVLHMEFSGRGDQVWISVRDADQLQ VWDPYRLKRIGSLPARSPSGIFFSHRAQHIGL

>P26474

MAIKITPDEFSLLIQRLNKKWRVFAPSAEFRGGRFSDTDNI IYQRISGWRDLIWHEKSHM 
SPNTIIAPITETLFYFDKDTIQIAETDTSPI I IFARACDINAMSRLDYMYLSNGNNSDYS YQLLREHIRFVLIECEESFENCFCVSMGTNKTDCYSAAMRFSDEGALVSIRDPFIEAAIQ GLGQEADYTPSFVSENRETVVTPDSVCHDPQKIRDILTHHPLWDAYDSRCISCGRCTTGC PTCTCYSVFDVAYDENPQRGERRRQWASCMVPGFSDMAGGHGFREKPGERLRYRALHKVN DYKARNGIEHMCVGCGRCDDRCPQYIKFSLI INKMTAAVRQALAEEA

$>Q 46877$

MSIVVKNNIHWVGQRDWEVRDFHGTEYKTLRGSSYNSYLIREEKNVLIDTVDHKFSREFV QNLRNEIDLADIDYIVINHAEEDHAGALTELMAQI PDTP IYCTANAIDS INGHHHHPEWN FNVVKTGDTLDIGNGKQLIFVETPMLHWPDSMMTYLTGDAVLFSNDAFGQHYCDEHLFND EVDQTELFEQCQRYYANILTPFSRLVTPKITEILGFNLPVDMIATSHGVVWRDNPTQIVE LYLKWAADYQEDRITIFYDTMSNNTRMMADA IAQGIAETDPRVAVKIFNVARSDKNEILT NVFRSKGVLVGTSTMNNVMMPKIAGLVEEMTGLRFRNKRASAFGSHGWSGGAVDRLSTRL QDAGFEMSLSLKAKWRPDQDALKLCREHGREIARQWALAPLPQSTVNTVVKEETSATTTA DLGPRMQCSVCQWIYDPAKGEPMQDVAPGTPWSEVPDNFLCPECSLGKDVFEELASEAK $>$ P2 6475

MSHCSCHDKPQHSLLPAAYRILSITRHTPLEWNFRVAVDFPAHWGQFVEVSLPRVGEAPI SVSDYGDGWIDLLIRNVGKVTSALFTLKEGDNVWLRGCYGNGYPVDTLRHKPLLVVAGGT GVAPVKGLMRYFVENPQEIGQLDMILGYKNRDCVLYKEEMATWRGKHNLVLTLDEGEADD RYQIGRVTDRLADMTLSDIDTMQAIVVGPPIMITFTVKMLLQKGLKPEQIWVDYERRMAC SVGKCGHCRMGEVYVCTDGPIFNYAVAQRFAD

$>$ P0A6P9

SKIVKI I GREI IDSRGNPTVEAEVHLEGGFVGMAAAPSGASTGSREALELRDGDKSRFLG KGVTKAVAAVNGP IAQALIGKDAKDQAGIDKIMIDLDGTENKSKFGANA I LAVSLANAKA AAAAKGMPLYEHIAELNGTPGKYSMPVPMMNI INGGEHADNNVDIQEFMIQPVGAKTVKE AIRMGSEVFHHLAKVLKAKGMNTAVGDEGGYAPNLGSNAEALAVIAEAVKAAGYELGKDI TLAMDCAASEFYKDGKYVLAGEGNKAFTSEEFTHFLEELTKQYP IVS IEDGLDESDWDGF AYQTKVLGDKIQLVGDDLFVTNTKILKEGIEKGIANS I L I KFNQIGSLTETLAAIKMAKD AGYTAVISHRSGETEDATIADLAVGTAAGQIKTGSMSRSDRVAKYNQLIRIEEALGEKAP YNGRKEIKGQA

$>$ P2 6477

MSIDRTSPLKPVSTVQTRETSDTPVQKTRQEKTSAATSASVTLSDAQAKLMQPGVSDINM ERVEALKTAIRNGELKMDTGKIADSLIREAQSYLQSK

>P33694

MLQILYLAQDLADPAVRRRTLTLVAGGARVTLAGFRRGDNPLAAIDGVEPIELGTTADGR FAQRIGAVARACLSLQRQLGHVRKPDVI IARNLEMLAVARRAVAFFGGTVPIVYECLDIH RLMLRKDIVGRMLRAAESQLGKDARLLITSSPAF IEHYFRPLSGIGAPPMLLENKVLEID GTVERRTASPAKSPPPGAPWKIGWFGALRCRRSLALLAEFSRKMEGRFEIVLRGRPAYSE FDDFDGFVRNEPFMRFEGAYRNPEDLAEIYGEVHFTWAIDFFEEGQNSAWLLPNRLYEGC RHGRIPIAMKGTETARFLSVRS IGLVLEGADVESLATVLGPLTPNCYADAAERISRCNPG SWVFDRTDCEALVRQLATLTLQAPQTVPVVAMAGSSHKEG

$>$ P33697

MNPPAIDKVPDVTFVVAAYNSADTIVRAIESALAQEGVTVEVVVVDDCSADATPALVAAI PDPRVRLIALDRNRGPGGARNAGIGAARGRWIAVLDSDDTVRPDRLRRMIERADAAGAQI AVDNLDVVSLDGRSLRMFSEAELARLPQLTLPAF IESNVLFRSEHNFGYMKP I FERRFLE NQQLRFDEALRIGEDYILLASALACGGRCAVEPSAGYIYHIREGS ISRVLRLDHIDAMIA ADEAFLRRYALDGLAQKMQHRRMRGFREARSFLVLVEQLKKRSLAGALKTALADPFALRH LSMP IAARLRRLAARFVHPSSHSAPRAAPVTAAAERSPLGNDPRISKG

$>$ P50176

MTAEKAEGATGFAGFDPKSVEPYIVKDPESLAINMARAAEQLGKAASAWLAPREAGEKTD SFAEPVSDMVKTLSKVSEYWLSDPRRTLEAQTHLLGSFFDMWSRTLQRMAGDAVEDPANL QRNDKRFADEDWVKNPFFDFIRQAYFVTSDWAERMVRDAEGLDDHTRHKAAFYVRQIASA LSPTNFITTNPQLYRETVASSGANLVKGMQMLAEDIAAGRGELRLRQTDTSKFAIGENIA ITPGKVIAQNDVCQVLQYEASTETVLKRPLLICPPWINKFYVLDLNPEKSF I KWAVDQGQ TVFVISWVNPDERHASKDWEAYAREGIGFALDI IEQATGEREVNS I GYCVGGTLLAATLA LHAAEGDERIRSATLFTTQVDFTHAGDLKVFVDDDQIRHLEANMSATGYLEGSKMASAFN MLRASELIWPYFVNNYLKGQDPLPFDLLYWNSDSTRMPAANHSFYLRNCYLENRLSKGEM VLAGRRVSLGDVKI PIYNLATKEDHIAPAKSVFLGSSSFGGKVTFVLSGSGHIAGVVNPP ARSKYQYWTGGAPKGDIETWMGKAKETAGSWWPHWQGWVERLDKRRVPARKAGGPLNS IE EAPGSYVRVRA 
$>$ P 78055

MELYLDTSDVVAVKALSRIF PLAGVTTNPS I IAAGKKPLDVVLPQLHEAMGGQGRLFAQV MATTAEGMVNDALKLRS I IADIVVKVPVTAEGLAAI KMLKAEGI PTLGTAVYGAAQGLLS ALAGAEYVAPYVNRIDAQGGSGIQTVTDLHQLLKMHAPQAKVLAASFKTPRQALDCLLAG CESITLPLDVAQQMISYPAVDAAVAKFEQDWQGAFGRTSI

$>$ P44406

MSKLVLILNCGSSSLKFAILDPATGEEKLSGLAEAFFLPEARIKWKLNGEKGNADLGAGA AHTEALNF IASNILNDELKNS IAAIGHR IVHGGEKYTQSVIVTDEVVKGIEDAAQFAPLH NPAHLIGIREAFKAFPHLKDKNVVVFDTAFHQTMPEEAFLYALPYSLYKEHGVRRYGAHG TSHYFVSREVAKYVGKPADQVNA I I CHLGNGGSVSVVRNGQCIDTSMGLTPLEGLVMGTR CGDIDPAIVFYLYKTLGMSMDQIEETLVKKSGLLGLTEVTSDCRYAEDNYDDESKPETRR ALNVYSYRLAKYIGAYMAVLGDDHLDAIAFTGGIGENSAHVRELALNHLKLFGIKIDNER NLATRFGKDGVITTDDSAFKAIVLPTNEELVIAQDTAKLC

$>$ P75726

MTQKIEQSQRQERVAAWNRRAECDLAAFQNSPKQTYQAEKARDRKLCANLEEAIRRSGLQ DGMTVSFHHAFRGGDLTVNMVMDVIAKMGFKNLTLASSSLSDCHAPLVEHIRQGVVTRIY TSGLRGPLAEEISRGLLAEPVQIHSHGGRVHLVQSGELNIDVAFLGVPSCDEFGNANGYT GKACCGSLGYAIVDADNAKQVVMLTEELLPYPHNPAS I EQDQVDLIVKVDRVGDAAKIGA GATRMTTNPRELLIARSAADVIVNSGYFKEGFSMQTGTGGASLAVTRFLEDKMRSRDIRA DFALGGITATMVDLHEKGLIRKLLDVQSFDSHAAQSLARNPNHIEISANQYANWGSKGAS VDRLDVVVLSALEIDTQFNVNVLTGSDGVLRGASGGHCDTAIASALS I IVAPLVRGRIPT LVDNVLTCITPGSSVDILVTDHGIAVNPARPELAERLQEAGIKVVSIEWLRERARLLTGE PQPIEFTDRVVAVVRYRDGSVIDVVHQVKE

$>$ P33771

MKPDAHHVKQFLLRLQDDI CQTLSAVDGANFVEDSWRREAGGGGRSRVLRNGGIFEQAGV NFSHVHGDAMPASATAHRPELAGRSFEAMGVSLVVHPHNPY I PTSHANVRFF IAEKPGAD PVWWFGGGFDLTPYYGFEEDAVHWHRTARDLCQPFGDDVYPRYKKWCDDYFFLKHRNEQR GVGGLFFDDLNTPDFDHCFDFMQAVGNGYTRAYLPIVERRKAMVWGERERNFQLYRRGRY VEFNLVWDRGTLFGLQTGGRTES ILMSMPPLVRWEYDWQPEAGSPEAALSEF IQVRDWI $>$ P04994

MLPSQSPAIFTVSRLNQTVRLLLEHEMGQVWISGEISNFTQPASGHWYFTLKDDTAQVRC AMFRNSNRRVTFRPQHGQQVLVRANITLYEPRGDYQI IVESMQPAGEGLLQQKYEQLKAK LQAEGLFDQQYKKPLPSPAHCVGVITSKTGAALHDI LHVLKRRDPSLPVI IYPAAVQGDD APGQIVRAIELANQRNECDVLIVGRGGGSLEDLWSFNDERVARAIFTSRIPVVSAVGHET DVTIADFVADLRAPTPSAAAEVVSRNQQELLRQVQSTRQRLEMAMDYYLANRTRRFTQIH HRLQQQHPQLRLARQQTMLERLQKRMSFALENQLKRTGQQQQRLTQRLNQQNPQPKIHRA QTRIQQLEYRLAETLRAQLSATRERFGNAVTHLEAVSPLSTLARGYSVTTATDGNVLKKV KQVKAGEMLTTRLEDGWIESEVKNIQPVKKSRKKVH

$>$ P69819

MTKI IAVTACP SGVAHTYMAAEALESAAKAKGWEVKVETQGS IGLENELTAEDVASADMV ILTKDIGIKFEERFAGKTIVRVNISDAVKRADAIMSKIEAHLAQTA

$>$ POAEDO

AYKHILIAVDLSPESKVLVEKAVSMARPYNAKVSLIHVDVNYSDLYTGLIDVNLGDMQKR ISEETHHALTELSTNAGYP ITETLSGSGDLGQVLVDA I KKYDMDLVVCGHHQDFWSKLMS SARQLINTVHVDMLIVPLRDEEE

$>$ P55913

MKFI IKLFPEITIKSQSVRLRFIKILTGNIRNVLKHYDETLAVVRHWDNIEVRAKDENQR LVIRDALTRIPGIHHILEVEDVPFTDMHDIFEKALAQYREQLEGKTFCVRVKRRGKHEFS SIEVERYVGGGLNQHIESARVKLTNPDVTVHLEVEDDRLLLIKGRYEGIGGFPIGTQEDV LSLISGGFDSGVSSYMLMRRGCRVHYCFFNLGGAAHEIGVRQVAHYLWNRFGSSHRVRFV AINFEPVVGEILEKVDDGQMGVVLKRMMVRAASKVAERYGVQALVTGEALGQVSSQTLTN LRLIDNVSDTLILRPLISYDKEHI INLARQIGTEDFARTMPEYCGVISKSPTVKAIKAKI EAEEENFDFS ILDKVVEEANNVDIREIAQQTQQEVVEVETVSGFGPNDVILDIRSVDEQD DKPLKVEGVDVVSLPFYKLSTKFGDLDQSKTWLLWCERGVMSRLQALYLREQGFANVKVY $\mathrm{RP}$

$>Q 04713$

MDFCLLNEKSQIFVHAEPYAVSDYVNQYVGTHS IRLPKGGRPAGRLHHRIFGCLDLCRIS YGGSVRVISPGLETCYHLQI ILKGHCLWRGYGQEHYFSPGELLLLNPDDQADLTYSEDCE KFIVKLPSVVLDRACSDNNWHKPREGIRFAARHNLQQLDGF INLLGLVCDEAEHTKSMPR 
VQEHYAGI IASKLLEMLGSNVSREIFSKGNPSFERVVQFIEENLKRNISLERLAELALMS PRSLYTLFEKHAGTTPKNYIRNRKLECIRARLSDPNANVRSVTEMALDYGFFHTGRFAEN YRSTFGELPSDTLRRRKMKWLDPEESLPPLP

$>$ P39662

MLTQKTKDIVKATAPVLAEHGYDI I KCFYQRMFEAHPELKNVFNMAHQEQGQQQQALARA VYAYAENIEDPNSLMAVLKNIANKHASLGVKPEQYP IVGEHLLAA I KEVLGNAATDDI IS AWAQAYGNLADVLMGMESELYERSAEQPGGWKGWRTFVIREKRPESDVITSF ILEPADGG PVVNFEPGQYTSVAIDVPALGLQQIRQYSLSDMPNGRTYRISVKREGGGPQPPGYVSNLL HDHVNVGDQVKLAAPYGSFHIDVDAKTPIVLISGGVGLTPMVSMLKVALQAPPRQVVFVH GARNSAVHAMRDRLREAAKTYENLDLFVFYDQPLPEDVQGRDYDYPGLVDVKQIEKS ILL PDADYYICGP I PFMRMQHDALKNLGIHEAR I HYEVFGPDL

$>$ POA9I3

TLSSQHYLVITALGADRPGIVNTITRHVSSCGCNIEDSRLAMLGEEFTFIMLLSGSWNAI TLIESTLPLKGAELDLLIVMKRTTARPRPPMPASVWVQVDVADSPHLIERFTALFDAHHM NIAELVSRTQPAENERAAQLHIQITAHSPASADAANIEQAFKALCTELNAQGS INVVNYS QHDEQDGVK

$>$ P0A6RO

MYTKI IGTGSYLPEQVRTNADLEKMVDTSDEWIVTRTGIRERHIAAPNETVSTMGFEAAT RAIEMAGIEKDQIGLIVVATTSATHAFPSAACQIQSMLGIKGCPAFDVAAACAGFTYALS VADQYVKSGAVKYALVVGSDVLARTCDPTDRGTI I IFGDGAGAAVLAASEEPGI ISTHLH ADGSYGELLTLPNADRVNPENS I HLTMAGNEVFKVAVTELAHIVDETLAANNLDRSQLDW LVPHQANLRI ISATAKKLGMSMDNVVVTLDRHGNTSAASVPCALDEAVRDGR I KPGQLVL LEAFGGGFTWGSALVRF

$>$ P 72324

MRTRAAVAVEAGKPLEIMEVNLEGPKAGEVMVEIKATGICHTDEFTLSGADPEGMFPAIL GHEGAGVVVEVGPGVTSVKPGDHVI PLYTPECRQCPSCLSQKTNLCTAIRGTQGQGLMPD GTSRFSMLDGTPILHYMGCSTFSNYTVLPEIAVAKVRPDAPFDKICYIGCGVTTGIGAVI NTAKVEIGAKAVVFGLGGIGLNVIQGLKLAGADMI IGVDLNNAKKEWGERFGMTHFVNPS EIDGDVVAHLVNMTKTPFDQIGGADYTFDCTGNVKVMRQALEACHRGWGQSIVIGVAPAG AEIQTRPFQLVTGRVWKGSAFGGARGRTDVPKIVDWYMEGKIQIDPMITHILSLEEINKG FDLMHAGESIRSVVVF

$>087386$

MSSYRLPKRGLVDRNVPLSFTFDGRPMQGLEGDTLASALLANGRMLVGRSFKYHRPRGIL TAGAAEPNALVTVGRGGRAEPNTRATMQELYEGLEARSQNRWPSLAFDIGALNGLLSPFL GAGFYYKTFMWPAPLWEKLYEPVIRRAAGLGKASYEADPDAYEKSWAHCDLLVIGAGPTG LAAALTAGRAGARVILVDEGSLPGGSLLSDTATIDGKAAADFARDTSDELRSMPNVQVLV RTTAFGWYDGNVFGAVERVQKHVREPASHLPVERLWRIVAGKALLATGAEERPLVFGGND RPGVMMAGAMRAYLNRYGVAPGRTPAIFTTNDTGYTLAQELEAAGVDVVAIVDSRPAAGV DYRGKARLVREAVVCGTKGGKAISAIEVHHGGRTETIAVDALAMAGGFDP I IHLACHRGG KPVWSAEKAAFLAPGSLKGLEVAGGAAATTGLAACLGEGAARAEAIVRELGLPCPPVAVV KVESEEGIRSPAPLWS I PGIKDKAFVDFQNDVHLKDIGLAVREGYSHVELAKRYTTSGMA TDQGKLSNVNAIGLIAKARGVSPAEVGTTTFRPFYTPVSFGALTGAHTGHHFQPVRKSPL HDWAKKHGAVFVETGLWYRSSWFPRSGERTWRESVEREVLNVRKNAGLCDVSMLGKIEIT GSDAAEFLNRVYCNAFLKLPVGKARYGLMLREDGF IYDDGTTSRLEENRFFMTTTTAYAA GVMNHLEFCAQVLWPQLDVRLAS ITDQWAQMA IAGPKARMILQKIVDEDISDAAFPFLAA KEVSLFGGALHGCLFRISFSGELAYELAVPAGYGESIADALLEAGKDHGIMPYGVETLSV LRIEKGHVTHNEINGTIVPADLGFGKMVSAGKPDFVGKAMLQREGLTAPDRPQLVGVVPL DPQQSFRSGSHILAKGAAATLENDEGYVTSSAYSPHVGSTIALALVRNGRNRHGEEVLVW SGLHGESTPARLCNPVFFDPQNERLHV

$>Q 59636$

MALQRTLSI I KPDAVSKNVIGEILTRFEKAGLRVVAAKMVQLSEREAGGFYAEHKERPFF KDLVSFMTSGPVVVQVLEGEDAIAKNRELMGATDPKKADAGTIRADFAVS IDENAVHGSD SEASAAREIAYFFAATEVCERIR

$>$ P56145

MKLS INDLNVFVNTPKDIAKLCEDLSRLGLEVESCI PCIAPKNVVVGKILEKAPHKNAEK LSVCQVDVGKEVLQIVCGAKNVAPNQFVPVALNGALIGSTTIAKTELRGVESHGMICSSI ELGFPKINDGILELDESVGELVLGKELNEYAPFNTHVLEISLTPNRGDCLSVLGIAREIS AFYHTPLKPIKALNFTPKSGLITLSAGEN IESHLAYYLICNHSLKTPLNIKLSLAHNNAL SENDLNNF IEFSTHFSGVIMNAYSLNTTPMDLSVKNDENNLESVYINHQKRSTIAIKHQV 
QKDLSECLLLEASYTDP ISLSLKLHALKDKTLQKDNALIYRSARGSNPNLSDGLNFLSAH LKATILESKQTEHSLKDRTLTFQLEDITEILGLAVEKEKIQGILKNLGFKVSVKEPNSKP QILEVIAPNFRHDIKTIQDIAEEILRFVGIDNLVSKPLHCVSSKNSNPNYDTHRFFENLK HKALACGFKEVIHYVFYSKEKQQKLGFEVLEDPLELQNPITTELNTLRTSLVCGLLDASL RNKNLGFKSIALYEKGSVYNSKREEIQKLGFLISGLQKKESYPDTKGKAWDFYSFAECVS KVIGDFSLEKLTTQTP INHPYQSAKI IQNHEI I GVIAKIHPKVIQELDLFESYYAEIDAF KLKRPAMLLKPFS IYPSSVRDLTLI IDENTAFSGIKKALKDAQI PNLSEILPLDIFKESN NSIALSVRCVIHSLEKTLNDEEVNSAVQKALEILEKEFNARLKG

$>$ P0A717

PDMKLFAGNATPELAQRIANRLYTSLGDAAVGRFSDGEVSVQINENVRGGDIFI IQSTCA PTNDNLMELVVMVDALRRASAGRITAVI PYFGYARQDRRVRSARVPITAKVVADFLSSVG VDRVLTVDLHAEQIQGFFDVPVDNVFGSPILLEDMLQLNLDNPIVVSPDIGGVVRARAIA KLLNDTDMA I IDKRRPRANVSQVMHI I GDVAGRDCVLVDDMIDTGGTLCKAAEALKERGA KRVFAYATHPIFSGNAANNLRNSVIDEVVVCDTIPLSDEIKSLPNVRTLTLSGMLAEAIR RISNEESISAMFEH

$>$ P38434

MAVNLTEKTAEQLPDIDGIALYTAQAGVKKPGHTDLTLIAVAAGSTVGAVFTTNRFCAAP VHIAKSHLFDEDGVRALVINTGNANAGTGAQGRIDALAVCAAAARQIGCKPNQVMPFSTG VILEPLPADKI IAALPKMQPAFWNEAARA IMTTDTVPKAASREGKVGDQHTVRATGIAKG SGMIHPNMATMLGF IATDAKVSQPVLQLMTQEIADETFNTITVDGDTSTNDSFVI IATGK NSQSEIDNIADPRYAQLKELLCSLALELAQAIVRDGEGATKFITVRVENAKTCDEARQAA YAAARSPLVKTAFFASDPNLGKRLAAIGYADVADLDTDLVEMYLDDILVAEHGGRAASYT EAQGQAVMSKDEITVRIKLHRGQAAATVYTCDLSHGYVSINADYRS

$>$ P44853

MSEQKQDVAATEEQQPVLQIQRIYVKDVSFEAPNLPHI FQQEWKPKLGFDLSTETTQVGD DLYEVVLNISVETTLEDSGDVAF I CEVKQAGVFTISGLEDVQMAHCLTSQCPNMLFPYAR ELVSNLVNRGTFPALNLSPVNFDALFVEYMNRQQAENAEEKSEEEQTKH

$>$ POA1R2

MLAKR I I PCLDVRDGQVVKGVQFRNHE I I GDIVPLAKRYADEGADELVFYDITASSDGRV VDKSWVARVAEVIDI PFCVAGGIRSIDDAAKILSFGADKIS INSPALADPTLITRLADRF GVQCIVVGIDTWFDDATGKYHVNQYTGDENRTRVTQWETLDWVQEVQQRGAGEIVLNMMN QDGVRNGYDLTQLKKVRDVCRVPLIASGGAGTMEHFLEAFRDADVDGALAASVFHKQI IN IGELKAYLAGQGVEIRIC

$>$ P44420

MKQYLELCRRIVSEGEWVANERTGKHCLTVINADLEYDVANNQFPLITTRKSYWKAAIAE FLGYIRGYDNAADFRALGTKTWDANANENAAWLANPHRRGVDDMGRVYGVQGRAWRKPNG ETIDQLRKIVNNLTKGIDDRGEILTFFNPGEFDLGCLRPCMHTHTFSLVGDTLHLTSYQR SCDVPLGLNFNQIQVFTFLALMAQITGKKAGKAYHKIVNAHIYEDQLELMRDVQLKREPF PLPKLEINPDIKTLEDLETWVTMDDFKVVGYQSHEPIKYPFSV

$>$ P07623

PIRVPDELPAVNFLREENVFVMTTSRASGQEIRPLKVLILNLMPKKIETENQFLRLLSNS PLQVDIQLLRIDSRESRNTPAEHLNNFYCNFEDIQDQNFDGLIVTGAPLGLVEFNDVAYW PQIKQVLEWSKDHVTSTLFVCWAVQAALNILYGI PKQTRTEKLSGVYEHHILHPHALLTR GFDDSFLAPHSRYADFPAALIRDYTDLEILAETEEGDAYLFASKDKRIAFVTGHPEYDAQ TLAQEFFRDVEAGLDPDVPYNYFPHNDPQNTPRASWRSHGNLLFTNWLNYYVYQITPYDL RHMNPTLD

$>$ P50199

MSHPDLFSLSGARALVTGASRGIGLTLAKGLARYGAEVVLNGRNAESLDSAQSGFEAEGL KASTAVFDVTDQDAVIDGVAAIERDMGP IDILINNAGIQRRAPLEEFSRKDWDDLMSTNV NAVFFVGQAVARHMI PRGRGKIVNI CSVQSELARPGIAPYTATKGAVKNLTKGMATDWGR HGLQINGLAPGYFATEMTERLVADEEFTDWLCKRTPAGRWGQVEELVGAAVFLSSRASSF VNGQVLMVDGGITVSL

$>$ P14916

MKLTPKELDKLMLHYAGELAKKRKEKGIKLNYVEAVALISAHIMEEARAGKKTAAELMQE GRTLLKPDDVMDGVASMIHEVGIEAMFPDGTKLVTVHTPIEANGKLVPGELFLKNEDITI NEGKKAVSVKVKNVGDRPVQIGSHFHFFEVNRCLDFDREKTFGKRLDIASGTAVRFEPGE EKSVELIDIGGNRRIFGFNALVDRQADNESKKIALHRAKERGFHGAKSDDNYVKTIKE $>$ P 60390

MMENYKHTTVLLDEAVNGLNIRPDGIYIDGTFGRGGHSRLILSQLGEEGRLLAIDRDPQA 
IAVAKTIDDPRFSI IHGPFSALGEYVAERDLIGKIDGILLDLGVSSPQLDDAERGFSFMR DGPLDMRMDPTRGQSAAEWLQTAEEADIAWVLKTYGEERFAKRIARAIVERNREQPMTRT KELAEVVAAATPVKDKFKHPATRTFQAVRIWVNSELEEIEQALKSSLNVLAPGGRLSI IS FHSLEDRIVKRFMRENSRGPQVPAGLPMTEEQLKKLGGRQLRALGKLMPGEEEVAENPRA RSSVLRIAERTNA

$>Q 4 \mathrm{~K} 530$

MARTTAINRYRNIGICAHVDAGKTTTTERILFYTGLSHKMGEVHDGAATTDWMVQEQERG ITITSAAVTTFWKGSRGQYDNYRVNVIDTPGHVDFTIEVERSLRVLDGAVVVFCGTSGVE PQSETVWRQANKYGVPRVVYVNKMDRAGANFLRVVGQ I KNRLGHTPVPVQLAIGAEDNFE GQVDLI KMKA I YWNDDDKGTTYREEE I PADMLDLANEWRSNMVEAAAEANEELMNKYLEE GDLTVEEIKAGLRARTLASEIVPAVCGSSFKNKGVPLVLDAVIDFLPAPTEI PAIKGIHP DLIEKPKDELVEADYDERHADDAEPFSALAFKIATDPFVGTLTFVRVYSGFLSSGDSVIN SVKGKKERVGRMVQMHANQREEI KEVRAGDIAAL I GMKDVTTGDTLCDLDKQ I I LERMDF PEPVISVAVEPKTKQDQEKMGIALGKLAQEDPSFRVKTDEETGQTI ISGMGELHLDILVD RMKREFNVEANIGKPQVSYREKISKSNVEIEGKFVRQSGGRGQFGHCWIRFSEPDVDANG NITEGLVFTNEVVGGVVPKEYIPAIQKGIEEQMKNGVVAGYPLIGLKATVFDGSYHDVDS NEMAFKVAASMATKQLAQKGGGVVLEPIMKVEVVTPEDYMGDVMGDLNRRRGLIQGMDDS VSGKVIRAEVPLGEMFGYATDVRSMSQGRASYSMEFSKYAEAPSNIVEALVKKQG

$>$ P0A6D0

MRSSAKQEELVKAFKALLKEEKFSSQGEIVAALQEQGFDNINQSKVSRMLTKFGAVRTRN AKMEMVYCLPAELGVPTTSSPLKNLVLDIDYNDAVVVIHTSPGAAQLIARLLDSLGKAEG ILGTIAGDDTIFTTPANGFTVKDLYEAILELFDQEL

$>$ P13039

MFEVTFWWRDPQGSEEYSTIKRVWVYITGVTDHHQNSQPQSMQRIAGTNVWQWTTQLNAN WRGSYCFI PTERDDIFSVPSPDRLELREGWRKLLPQAIADPLNLQSWKGGRGHAVSALEM PQAPLQPGWDCPQAPEI PAKEI IWKSERLKKSRRVWI FTTGDATAEERPLAVLLDGEFWA QSMPVWPVLTSLTHRQQLPPAVYVLIDAIDTTHRAHELPCNADFWLAVQQELLPLVKAIA PFSDRADRTVVAGQSFGGLSALYAGLHWPERFGCVLSQSGSYWWPHRGGQQEGVLLEKLK AGEVSAEGLRIVLEAGIREPMIMRANQALYAQLHP I KES I FWRQVDGGHDALCWRGGLMQ GLIDLWQPLFHDRS

$>$ P0A7A5

VSRRVQALLDQLRAQGIQDEQVLNALAAVPREKFVDEAFEQKAWDNIALPIGQGQTISQP YMVARMTELLELTPQSRVLEIGTGSGYQTAILAHLVQHVCSVERIKGLQWQARRRLKNLD LHNVSTRHGDGWQGWQARAPFDA I IVTAAP PE I PTALMTQLDEGGILVLPVGEEHQYLKR VRRRGGEFIIDTVEAVRFVPLVKGELA

$>$ P32055

MSKQRVFIAGHRGMVGSAIRRQLEQRGDVELVLRTRDELNLLDSRAVHDFFASERIDQVY LAAAKVGG IVANNTYPADF I YQNMMI ESNI I HAAHQNDVNKLLFLGSSCIYPKLAKQPMA ESELLQGTLEPTNEPYAIAKIAGIKLCESYNRQYGRDYRSVMPTNLYGPHDNFHPSNSHV IPALLRRFHEATAQNAPDVVVWGSGTPMREFLHVDDMAAAS IHVMELAHEVWLENTQPML SHINVGTGVDCTIRELAQTIAKVVGYKGRVVFDASKPDGTPRKLLDVTRLHQLGWYHEIS LEAGLASTYQWFLENQDRFRG

$>$ P11295

MKKSVDFIGVGTGPFNLSIAALSHQIEELDCLFFDEHPHFSWHPGMLVPDCHMQTVFLKD LVSAVAPTNPYSFVNYLVKHKKFYRFLTSRLRTVSREEFSDYLRWAAEDMNNLYFSHTVE NIDFDKKRRLFLVQTSQGEYFARNICLGTGKQPYLPPCVKHMTQSCFHASEMNLRRPDLS GKRITVVGGGQSGADLFLNALRGEWGEAAEINWVSRRNNFNALDEAAFADEYFTPEYISG FSGLEEDIRHQLLDEQKMTSDGITADSLLTIYRELYHRFEVLRKPRNIRLLPSRSVTTLE SSGPGWKLLMEHHLDQGRESLESDVVIFATGYRSALPQILPSLMPLITMHDKNTFKVRDD FTLEWSGPKENNIFVVNASMQTHGIAEPQLSLMAWRSARILNRVMGRDLFDLSMPPALIQ WRSGT

$>$ P42321

MEKKKLTTAAGAPVVDNNNVITAGPRGPMLLQDVWFLEKLAHFDREVIPERRMHAKGSGA FGTFTVTHDITKYTRAKIFSEVGKKTEMFARFSTVAGERGAADAERDIRGFALKFYTEEG NWDMVGNNTPVFYLRDPLKF PDLNHIVKRDPRTNMRNMAYKWDFFSHLPESLHQLTIDMS DRGLPLSYRFVHGFGSHTYSF INKDNERFWVKFHFRCQQGI KNLMDDEAEALVGKDRESS QRDLFEAIERGDYPRWKLQIQIMPEKEASTVPYNPFDLTKVWPHADYPLMDVGYFELNRN PDNYFSDVEQAAFSPANIVPGISFSPDKMLQGRLFSYGDAHRYRLGVNHHQI PVNAPKCP FHNYHRDGAMRVDGNSGNGITYEPNSGGVFQEQPDFKEPPLSIEGAADHWNHREDEDYFS 
QPRALYELLSDDEHQRMFARIAGELSQASKETQQRQIDLFTKVHPEYGAGVEKAI KVLEG KDAK

$>$ P 69330

MKINQPAVAGTLESGDVMIRIAPLDTQDIDLQINSSVEKQFGDAIRTTILDVLARYNVRG VQLNVDDKGALDCILRARLEALLARASGIPALPWEDCQ

$>$ Q82S73

MTIDHEVKRRRTFAI ISHPDAGKTTLTEKLLLFAGAIHIAGSVKARKASRHATSDWMEIE KQRGISVASSVMQMEYRDCVINLLDTPGHQDFSEDTYRVLTAVDAALMVIDAANGVESQT LRLLQVCRARNTP I ITFVNKLDREVREPLDLIDEIERTLGMDVI PFTWPVGSGKRFHGVY DLRHKLMRVFRAGMDRVEQEETAI ITNLEDPAISERFGANLEQARQEIELITGAAPEFDQ TAFLAGQQTPVFFGSAINNFGVQEVLDTLVELAPPPGSRKAIQREIQPAEKKFSGVVFKI QANMNPAHRDRIAFVRICSGEFRRGMNLKVVRSGKDVRTSTVVSFLSQRRELLETAYAGD I IGI PNHGTLQLADTLTEGDHLQFTGLPFFAPEI FQTVEIADPLRSKQLKLGLAQLGEEG AIQVFRPHIGSMLLLGAVGVLQFEVVTHRLKHEYGVEARIAPAKYQLARWVTAETPQELQ RFIDANAHRIAYDAVNAPTFLASFSAEISVAEENWPGIRFHKMREHAGLMFQTAG

$>$ P16525

MARYDLVDRLNTTFRQMEQELAIFAAHLEQHKLLVARVFSLPEVKKEDEHNPLNRIEVKQ HLGNDAQSLALRHFRHLFIQQQSENRSS KAAVRLPGVLCYQVDNLSQAALVSHIQHINKL KTTFEHIVTVESELPTAARFEWVHRHLPGLITLNAYRTLTVLHDPATLRFGWANKH I I KN LHRDEVLAQLEKSLKSPRSVAPWTREEWQRKLEREYQDIAALPQNAKLKIKRPVKVQPIA RVWYKGDQKQVQHACPTPLIALINRDNGAGVPDVGELLNYDADNVQHRYKPQAQPLRLI I PRLHLYVAD

$>$ POA 229

MSQWQNICKIDDILPGTGVCALSGGEQVAIFRPYHSDQVFAISNIDPFFEASVLSRGLIA EHQGELWVASPLKKQRFRLSDGLCMEDEQFSVKHYDARVKDGVVQLRG

$>$ P61417

MNITLTKRQQEFLLLNGWLQLQCGHAERACILLDALLTLNPEHLAGRRCRLVALLNNNQG ERAEKEAQWLISHDPLQAGNWLCLSRAQQLNGDLDKARHAYQHYLELKDHNESP

$>$ P27888

MLNINFVNEESSTNQGLIVF IDEQLKLNNNLIALDQQHYELISKTIQNKLQFSGNYGQIT VVPSVIKSCAVKYLI I VGLGNVEKLTEAKIEELGGKI LQHATCAKIATIGLKI INR INRF TSPTFTSLIASGAFLASYRFHKYKTTLKEVEKFAVESIEILTDNNSEAMKLFEVKKLIAE AVFFTRDISNEPSNIKTPQVYAERIVEILEPLGVNIDVIGEHDI KNLGMGALLGVGQGSQ NESKLVVMEYKGGSRDDSTLALVGKGVIFDTGGISLKPSSNMHLMRYDMAGSAAVVGTI I ALASQKVPVNVVGVVGLVENMQSGNAQRPGDVVVTMSGQTAEVLNTDAEGRLVLADTVWY VQEKFNPKCVIDVATLTGAITVALGSTYAGCFSNNDELADKLIKAGEAVNEKLWRMPLHD DYDAMINSDIADIANIGNVPGAAGSCTAAHF I KRF I KDGVDWAHLDIAGVANSNNASALC PKGAVGYGVRLLEKFIKEYN

$>$ Q87DU6

MSEITTTDAQAQPEKKDFIRQI IREDLAHGTHTHIHTRFPPEPNGYLHIGHAKAICLDFG VAAEFGGHCTLRMDDTNPSKEDPAFAAAIQEDVSWLGFHWNALRHTSDYFEVLYLAAEKL IADGKAYVCDLNSQQVREYRGTLTEAGRPSPWRERSPDENLELFRQMRAGTFPDGTRTLR AKIDMASGNINLRDPALYRI KHVEHQNTGNTWPIYPMYDFAHALSDAIEGITHSLCTLEF EDHRPLYDWCINHVDLPNNSHLLKPLLDKGFPQEPSQPRQIEFSRLNINYTVMSKRKLTA LVDEKLVEGWDDPRMYTLQGLRRRGYTPAAMRLFVERIGISKQNS I IDFSVLENCLRENL DTIAPRRMATIAPMKLVLTNLAEDHEEQLIFPNHPKDDTQGTRTVPFSRELWIERDDFSE APPKGWKRLIPGGEVRLRGAGIARIDEVVKNAEGHVIALHGWLDPTSRPGMEGAHRKVKG TIHWVSAPHAVAAEIRLYDRLFS IEKPDDNTDGKTYRDFLNPDSKRVVHGYIEPAAAQTA PEHAFQFERLGYFVTDRHDHDATHPVFNRSVTLRDTWQRD

$>$ Q6MI59

MPLVEGPLYRFCYGEYKNMVFDCNGTICKSGAHEQGRHMKFVKELKRTHYCGSLGVSQAG QKVVLMGWVDVRRDHGSLVFIDLRDREGIVQVVLDPNKAETASSKNLRGEFVLAVEGVVR ARPDGMKNAKI KTGEVEVEAIRCEILNESAVPPFQVSDTNVNEMLRLKYRYLDLRSARLS SHLITRHKVAQLVRRFLSDNGFLEVETPILYKSTPEGARDYLVPSRVNPGHFYALPQSPQ TLKQLLMISGYDRYFQIARCFRDEDLRADRQPEFSQIDMEMSYIDQEDIMEMNEKLLRTI WKEIKGIDVGA I PRMTYQEAMDRYGIDKPDTRFGVEI KDLKSIVTGSGFKVFDDVLARGG IVRGIAAPKGGSYSRGQLDKLTDMAKRAGAKGLVWI KSEADGTLSSSVSKFFSPEKLAEM FKACGGEAGDCALVVADDYDTACAALSTLRLHLGRELNLIDNS KYKFLWVVDFPLLEYSP DEKRWVARHHPFTS PKDEFAQDLVNNNEAAYGKMLAKAYDLVCNGYEMGGGS I RIYRNEI 
QQAMFRLLGMSEEETQHKFGFFLEALKYGTPPHGGIAWGMDRLVMLLCETDAIREVIAFP KTAKATDLMSDCPSEVNRDQLAEVGVRLSTLAEKHLEDLKKS

$>$ P0A9K7

MDSLNLNKHISGQFNAELES IRTQVMTMGGMVEQQLSDAITAMHNQDSDLAKRVIEGDKN VNMMEVAIDEACVRI IAKRQPTASDLRLVMVISKTIAELERIGDVADKICRTALEKFSQQ HQPLLVSLESLGRHTIQMLHDVLDAFARMDIDEAVRIYREDKKVDQEYEGIVRQLMTYMM EDSRTIPSVLTALFCARS IERIGDRCQNI CEF IFYYVKGQDFRHVGGDELDKLLAGKDSD $\mathrm{K}$

$>$ POA 9 K9

MKVAKDLVVSLAYQVRTEDGVLVDESPVSAPLDYLHGHGSLISGLETALEGHEVGDKFDV AVGANDAYGQYDENLVQRVPKDVFMGVDELQVGMRFLAETDQGPVPVEITAVEDDHVVVD GNHMLAGQNLKFNVEVVAIREATEEELAHGHVHGAHDHHHDHDHDGCCGGHGHDHGHEHG GEGCCGGKGNGGCGCH

$>$ P0A731

MELTTRTLPARKHIALVAHDHCKQMLMSWVERHQPLLEQHVLYATGTTGNLISRATGMNV NAMLSGPMGGDQQVGALISEGKIDVLIFFWDPLNAVPHDPDVKALLRLATVWNI PVATNV ATADFIIQSPHFNDAVDILIPDYQRYLADR

$>$ P0A6T1

MKNINPTQTAAWQALQKHFDEMKDVTIADLFAKDGDRFSKFSATFDDQMLVDYSKNRITE ETLAKLQDLAKECDLAGA I KSMF SGEKINRTENRAVLHVALRNRSNTPILVDGKDVMPEV NAVLEKMKTF SEA I ISGEWKGYTGKA ITDVVNIGIGGSDLGPYMVTEALRPYKNHLNMHF VSNVDGTHIAEVLKKVNPETTLFLVASKTFTTQETMTNAHSARDWFLKAAGDEKHVAKHF AALSTNAKAVGEFGIDTANMFEFWDWVGGRYSLWSAIGLS IVLS I GFDNFVELLSGAHAM DKHFSTTPAEKNLPVLLALIGIWYNNFFGAETEA ILPYDQYMHRFAAYFQQGNMESNGKY VDRNGNVVDYQTGP I IWGEPGTNGQHAFYQLIHQGTKMVPCDF IAPAITHNPLSDHHQKL LSNFFAQTEALAFGKSREVVEQEYRDQGKDPATLDYVVPFKVFEGNRPTNSILLREITPF SLGALIALYEHKI FTQGVI LNIFTFDQWGVELGKQLANR I LPELKDDKE I SSHDSSTNGL INRYKAWRG

$>$ P0A7B8

TTIVSVRRNGHVVIAGDGQATLGNTVMKGNVKKVRRLYNDKVIAGFAGGTADAFTLFELF ERKLEMHQGHLVKAAVELAKDWRTDRMLRKLEALLAVADETASLI ITGNGDVVQPENDLI AIGSGGPYAQAAARALLENTELSAREIAEKALDIAGDI CIYTNHFHTIEELSYKA $>$ Q7VZ05

MNTRVLTGITTTGTPHLGNYAGAIRPAIQASTQPGVDAFFFLADYHALIKCDDPARVARS RLELAATWLAAGLDPERVTFYRQSDI PEITELCWLLTCVTPKGLMNRAHAYKASVDQNAA KGVEPDDGVTMGLFSYPVLMAADILLFNANQVPVGRDQVQHLEMARDIAQRFNHLYGREF FVLPEVVIAEEVATLPGLDGRKMSKSYNNTI PLFEGGAAGLRNATQR IVTDSRLPGEPKD AEASHLYMLYRAF STQQESMAFRRQLEEGMGWGDAKQALYERLERDLAPMRERYVELISN PGLIEDILQVGAAKARKLAQPLVRTLRDAVGLGVLQPAAAKAAQPARKAAKDARFVSFRD EDGSFRFRLLAADGEELLCSVPFANPKEAGALMRRLQDEAPEQALRGHDDVSYAAWLDGK EVAYGPQAADAGARDALLAKAREALAQLAA

$>$ Q6MNT9

MKIKHLEDLNTLVSLSKRRGFVFQSSEIYGGLGSCWDYGPLGSLMKLNVKRAWWNAMTRR PDIVGLDAAILMHPMVWKASGHVDGFSDPLVDCKECKTRFRADNTDSYINEKKCPNCGSK NLSEERSFNLMFKTHMGPLEDSGSVVYLRPETAQGHFVNFQNCQQASRYKIPFGIAAIGK SFRNEITPGNF IFRTREFEQMEMQYFVEPGTDEQFFKEWKERRWNFYIKFGIKPENLKFK DHDKLAHYAKAAVDVEFKFPMGFSELEGIHNRSDFDLSQHMKFSGKNLEYFDEPNKKKYI PYVIETAVGCDRLFLAFLCDAYREEVTTDEAGKEDVRVVMGLHPEIAPFKVAVLPLSKKE ELSSISEKLRDQLAEDFDVNYDESQSIGKRYRRQDEIGTPFCVTVDFDTINDQAVTVRHR DKMTQERVAITQLNAYIAAKLKTFNQ

$>$ P 38380

MLGFAKKLFGSSNERKVKTLATRVAKINAYEAEYAALSDEALKGKTAEFKARLEKGETLD DILNEAFAVVREASKRVLGMRHFDVQMVGGMVLHFSGISEMRTGEGKTLVATLPTYLNAL EGKGVHVITVNDYLARRDADWMGQVYNFLGLSYGVIVNGLSQGERQRAYRSDITYGTNNE FGFDYLRDNLVYSVDEMVQRGHNFAIVDEVDS ILIDEARTPLI ISGPTEDRSSFYKTIDV LVKELILDKSMFDHDEKQKQVILTEDGQEKIEEI LMSGGHLAEDSAGLYDAANVSVVHHV NQALRANILYTRDKDYIVKGGEVVLIDEFTGRMMTGRRLSEGLHQAIEAKEGADIQPENQ TLASVTIQNYFRLYKKLSGMTGTASTEAQEFDDIYKMSVSEIPTNRTIQRIDDDDEVYRT EREKNEAILKQIADCHVRGQPILVGTVSIEKSEELSKLLSTFSFEKDGKKVKGI PHQVLN 
ARFHEQEAVIVADAGVPGAVTIATNMAGRGTDIQLGGS IDMRLFNWRQQQRGMGLEITVE DEAEERARLETEIADKKAQALAAGGLFVLGTERHESRR IDNQLRGRTGRQGDPGRSKFFL SCEDDLLRIFAGERLDAIMRTFGVQEGEAITHKWLNNAIATAQKRVEQRNYEIRKNLLKY DDVVNDQRKAVFEQRQEFMESSDLSDI I HEMRRDVIDDLVLRHLPPKAYAEQWDVEGLTE RVKS ILGLDLPIAEWAAEEGIADEEMKERITKAADEYAAQREVI ITPEQMRSVEKSFLLQ MIDLQWREHLMHLDHLRNVIGLRGYGQRDPLNEYKTEAFSLFEKLLGDLRTNTTRWLMTV EIAYAEPEVPHTPLDNLVEVHLDPLTGENAAFAGGI PEGLSTAQREALPVSALPEGWDRT NRNAPCPCGSGKKFKQCHGSLVR

$>$ Q92H06

MNNNVITRFAPSPTGFLHIGSARTALFNYLFARHHNGKFLLRIEDTDKERSTKEAVEAIF SGLKWLGLDWNGEVI FQSKRNNLYKEAALKLLQNGKAYYCFTRQEEIERQRQQALENKQH FIFNSEWRDKDPS IYPTDI KPVIRLKTPREGS IT I HDTLQGEVVIENSH IDDMVLLRADG TATYMLAVVVDDHDMGITHI I RGDDHLTNTARQLA I YQAFGYAVPSMTH I PL I HGADGAK LSKRHGALGIEAYKDMGYLPESLCNYLLRLGWSHGDDEI I SMTQAIDWFNLDSLGKSPSK LDFAKMNSLNAHYLRMLDNDSLTSKTVEILEQNYNTLLRHLPYREEFGGNTERSTAAYID IREDASTGLTYKLPLAVELPKKFKISEQEIGY I KQAMP SLLVRSETLLELTRLAQIYLVD SPI IYSQDSKEI I ENCDKNL I KQI I ENLSELEQFDKESVQNKFKEIAAANDLKLNDIMKP VRALITGMTASPSIFEIAEILGKENILKRLKII

$>$ POA2Q4

MIEI KHLKTLQALRNSGSLAAAAAVLHQTQSALSHQFSDLEQRLGFRLFVRKSQPLRFTP QGEVLLQLANQVLPQISRALQACNEPQQTRLRIAIECHSCIQWLTPALENFRASWPQVEM DFTSGVTFDPQPALQQGELDLVMTSDILPRSGLHYSPMFDFEVRLVLAPDHPLASKTQIT PEDLASETLLIYPVQRSRLDVWRHFLQPAGISPLLKSVDNTLLLIQMVAARMGIAALPHW VVESVERQGLVVTKTLGDGLWSRLYAAVRDGDQRQAVTEAFIRSTRDHACDHLPFVRSAE RPIFDAPTAKPGSQPRL

$>$ P11880

MISVTLSQLTDILNGELQGADITLDAVTTDTRKLTPGCLFVALKGERFDAHDFADQAKAG GAGALLVSRPLDIDLPQLIVKDTRLAFGELAAWVRQQVPARVVALTGSSGKTSVKEMTAA ILSQCGNTLYTAGNLNNDIGVPMTLLRLTPEYDYAVIELGANHQGEIAWTVSLTRPEAAL VNNLAAAHLEGFGSLAGVAKAKGEIFSGLPENGIAIMNADNNDWLNWQSVIGSRKVWRFS PNAANSDFTATNIHVTSHGTEFTLQTPTGSVDVLLPLPGRHNIANALAAAALSMSVGATL DAI KAGLANLKAVPGRLFPIQLAENQLLLDDSYNANVGSMTAAVQVLAEMPGYRVLVVGD MAELGAESEACHVQVGEAAKAAGIDRVLSVGKQSHAISTASGVGEHFADKTALITRLKLL IAEQQVITILVKGSRSAAMEEVVRALQENG

$>$ P12758

SKSDVFHLGLTKNDLQGATLAIVPGDPDRVEKIAALMDKPVKLASHREFTTWRAELDGKP VIVCSTGIGGPSTSIAVEELAQLGIRTFLRIGTTGAIQPHINVGDVLVTTASVRLDGASL HFAPLEFPAVADFECTTALVEAAKS IGATTHVGVTASSDTFYPGQERYDTYSGRVVRHFK GSMEEWQAMGVMNYEMESATLLTMCASQGLRAGMVAGVIVNRTQQEI PNAETMKQTESHA VKIVVEAARR

$>$ P50286

MAKPQVTILATGGTIAGSGESSVKSSYSAGAVTVDKLLAAVPA INDLATI KGEQISSIGS QEMTGKVWLKLAKRVNELLAQKETEAVI ITHGTDTMEETAFFLNLTVKSQKPVVLVGAMR SGSSMSADGPMNLYNAVNVAINKASTNKGVVIVMNDE I HAAREATKLNTTAVNAFASPNT GKIGTVYYGKVEYFTQSVRPHTLASEFDISKIEELPRVDILYAHPDDTDVLVNAALQAGA KGI IHAGMGNGNPFPLTQNALEKAAKSGVVVARSSRVGSGSTTQEAEVDDKKLGFVATES LNPQKARVLLMLALTKTSDREAIQKIFSTY

$>$ P42195

MARYLEVSDIVQQWRNERPDLDVEPMLVIGTLSRVSLLIDRALDKVFSKYKLSAREFDIL ATLRRRGAPYAYSPSQIVNALMINNSTLTSRLDRLEQAGWLRRMPIEGDRRSVNIQLTDE GFALINRVVEEHVENERDILSPFSEEEKTHLRALLGRVEKHLVNNR

$>$ P11886

MQPIRLGLVGYGKIAQDQHVPAINANPAFTLVSVATQGKPCPGVENFQSLGELLENGPPV DAIAFCTPPQGRFALVQQALAAGKHVLVEKPPCATLGKAALWI KREQASAPCSPCIAYAP AIAAARDWLATRTLQSVQIDWKEDVRKWHPGQAWIWQPGLGVFDPGINALSIVTHLLPLP LFVESAELRVPSNCQSPIAAS I KMSDPRLLDVRAEFDFDHGHDELWSIQIRCAEGTLRLD NGGALLS IDGVRQTVAEEGEYAAVYRHFQQLIGDKTSDVDVQPLRLVADSFFVGSRVSVE $>$ P44442

MSKWDSNI PFLFFSNKKITMIKECQNPPHFRVVTDNTALLEVCNLAQQKSAVALDTEFMR 
VSTYFPKLGLIQLYDGEHVSLIDPLAITDFSPFVALLANPKVLKILHSCSEDLLVFLQEF DQLPRPMIDTQIMARFLGLGTSAGLAKLAQQYLNVEIDKGATRTNWI KRPLSDIQLQYAA GDVWYLLPLYHILEKELAKTPWEQAVRDDCELVLAKTHKLQERDSEKAYLDI PNAWKLNP LELSRLRVLAQWRQNVAIERDLALSYIVKSEHLWKVAKNNPRNTSEMLEMGLTENEVRVR GKEILQLLSQARRISSNDYPKS IERISEDPRYKKTIRLLQEKVNSLTPEGLTPEIVASKR TLEELIKWVWKYDCSQDKRPELLIGWRKPIGEKLVDALK

$>$ P37081

MQITLAR IDDRLI HGQVTTVWSKVANAQR I I I CNDDVFNDEVRRTLLRQAAPPGMKVNVV SLEKAVAVYHNPQYQDETVFYLFTNPHDVLTMVRQGVQIATLNIGGMAWRPGKKQLTKAV SLDPQDIQAFRELDKLGVKLDLRVVASDPSVNILDKINETAFCE

$>085014$

MSSNTSAPSLNALAGPLVESLVADAAKLRLIVAQENGARTVDAGANARGS IEAGRRIAEI CLGGLGTVTIAPIGPVASWPYTVVVHSADPVLACLGSQYAGWSLADEEGDSGFFALGSGP GRAVAVVEELYKELGYRDNATTTALVLESGSAPPASVVNKVAAATGLAPENVTFIYAPTQ SLAGSTQVVARVLEVALHKAHTVGFDLHKILDGIGSAPLSPPHPDF IQAMGRTNDA I IYG GRVQLFVDADDADAKQLAEQI PSTTSADHGAPFAEIFSRVNGDFYKIDGALFSPAEAIVT SVKTGKSFRGGRLEPQLVDASFV

$>$ Q9S4 49

MADQKQRVTVIGGGLAGTECAYQLSRRGVPVVLREMKPQKRSPAHKSDTLAELVCSNSLR SDNPESAIGLLHAELRALGSLVLSAADANRVPAGDALAVERERFSAAITESLLRQPGVEL VAGEVDELPEDGPVVIATGPLTSDALTRELERHVGTRLYFYDS IAP I LSADS IDMNVAFR QSRYGKGGGDDYLNLPMTKDEYYRF IAEVKAGQKVVPHAFEEPKSFEGCLPIEVMAERGD NTLAYGPMKPVGLRDPRTGHEPYAVVQLLMEDVGGTSWNMVGFQTRLTWGEQKRIFSSFI PGLQQAEFLRMGQMHRNTF IDSPRLLAKDLSLKTEPRLYFAGQISGVEGYVESAACGYLV ALALHARLTGTEFVPPPATTAMGALLRHVTGEAHP PDYPHQPSNISFGIFSPLTGRMKKA EKRAAYSARAKQDLAAWLPHAGVPAAGAPEHVDQRSA

$>$ P33012

MNYEIKQEEKRTVAGFHLVGPWEQTVKKGFEQLMMWVDSKNIVPKEWVAVYYDNPDETPA EKLRCDTVVTVPGYFTLPENSEGVILTEITGGQYAVAVARVVGDDFAKPWYQFFNSLLQD SAYEMLPKPCFEVYLNNGAEDGYWDIEMYVAVQPKHH

$>$ POA9L8

MEKKIGFIGCGNMGKAILGGLIASGQVLPGQIWVYTPS PDKVAALHDQFGINAAESAQEV AQIADI IFAAVKPGIMIKVLSEITSSLNKDSLVVS IAAGVTLDQLARALGHDRKI IRAMP NTPALVNAGMTSVTPNALVTPEDTADVLNIFRCFGEAEVIAEPMIHPVVGVSGSSPAYVF MFIEAMADAAVLGGMPRAQAYKFAAQAVMGSAKMVLETGEHPGALKDMVCSPGGTTIEAV RVLEEKGFRAAVIEAMTKCMEKSEKLSKS

$>$ P11458

MSVMFDPDTAIYPFPPKPTPLSIDEKAYYREKIKRLLKERNAVMVAHYYTDPEIQQLAEE TGGCISDSLEMARFGAKHPASTLLVAGVRFMGETAKILSPEKTILMPTLQAECSLDLGCP VEEFNAFCDAHPDRTVVVYANTSAAVKARADWVVTSS IAVELIDHLDSLGEKI IWAPDKH LGRYVQKQTGGDILCWQGACIVHDEFKTQALTRLQEEYPDAAILVHPESPQAIVDMADAV GSTSQLIAAAKTLPHQRLIVATDRGI FYKMQQAVPDKELLEAPTAGEGATCRSCAHCPWM AMNGLQAIAEALEQEGSNHEVHVDERLRERALVPLNRMLDFAATLRG

$>$ P0A6F5

AAKDVKFGNDARVKMLRGVNVLADAVKVTLGPKGRNVVLDKSFGAPTITKDGVSVAREIE LEDKFENMGAQMVKEVASKANDAAGDGTTTATVLAQA I ITEGLKAVAAGMNPMDLKRGID KAVTAAVEELKALSVPCSDSKAIAQVGTISANSDETVGKLIAEAMDKVGKEGVITVEDGT GLQDELDVVEGMQFDRGYLSPYFINKPETGAVELESPFILLADKKISNIREMLPVLEAVA KAGKPLLI IAEDVEGEALATLVVNTMRGIVKVAAVKAPGFGDRRKAMLQDIATLTGGTVI SEEIGMELEKATLEDLGQAKRVVINKDTTTI IDGVGEEAA IQGRVAQIRQQIEEATSDYD REKLQERVAKLAGGVAVIKVGAATEVEMKEKKARVEDALHATRAAVEEGVVAGGGVALIR VASKLADLRGQNEDQNVGI KVALRAMEAPLRQIVLNCGEEPSVVANTVKGGDGNYGYNAA TEEYGNMIDMGILDPTKVTRSALQYAASVAGLMITTECMVTDLPKNDAADLGAAGGMGGM GGMGGMM

$>$ P69783

GLFDKLKSLVSDDKKDTGTIEI IAPLSGEIVNIEDVPDVVFAEKIVGDGIAIKPTGNKMV APVDGTIGKIFETNHAFSIESDSGVELFVHFGIDTVELKGEGFKRIAEEGQRVKVGDTVI EFDLPLLEEKAKSTLTPVVISNMDEIKELIKLSGSVTVGETPVIRIKK

$>$ P36936 
MELLLLSNSTLPGKAWLEHALPLIANQLNGRRSAVFI PFAGVTQTWDEYTDKTAEVLAPL GVNVTGIHRVADPLAAIEKAEI I IVGGGNTFQLLKESRERGLLAPMADRVKRGALYIGWS AGANLACPTIRTTNDMPIVDPNGFDALDLFPLQINPHFTNALPEGHKGETREQRIRELLV VAPELTVIGLPEGNWIQVSNGQAVLGGPNTTWVFKAGEEAVALEAGHRF

$>$ P37596

MSNGIVI IGSGFAARQLVKNIRKQDATI PLTLIAADSMDEYNKPDLSHVISQGQRADDLT RQTAGEFAEQFNLHLFPQTWVTDIDAEARVVKSQNNQWQYDKLVLATGASAFVPPVPGRE LMLTLNSQQEYRACETQLRDARRVLIVGGGLIGSELAMDFCRAGKAVTLIDNAAS ILASL MPPEVSSRLQHRLTEMGVHLLLKSQLQGLEKTDSGIQATLDRQRNIEVDAVIAATGLRPE TALARRAGLTINRGVCVDSYLQTSNTDIYALGDCAEINGQVLPFLQP IQLSAMVLAKNLL GNNTPLKLPAMLVKIKTPELPLHLAGETQRQDLRWQINTERQGMVARGVDDADQLRAFVV SEDRMKEAFGLLKTLPM

$>Q 8 Y 211$

MLTFQQLILKLQSYWDAQGCALLQP IDLEVGAGTSHVHTFLRAIGPEPWRAAYVQPSRRP KDGRYGENPNRLQHYYQYQVVLKPAPENILDLYLGSLQALGLDLKQNDVRFVEDDWENPT LGAWGLGWEVWLNGMEVTQFTYFQQVGGLDCKPITGEITYGIERLAMYLQQVENVYDLVW TEWEEPGPNGPVKRRLTYGDVYHQNEVEQSTYNFEHADTAVLFRRFAEHEAEAKRLMGVR EEGAADDGAAVPQLALPAYEQVLKAGHTFNLLDARGA ISVTERAAY IGRIRNLSRLVAQA YYDSRERLGFPMCGTAAAPAATEAA

$>$ P4 4527

MQTKYDLSTMF IHSGRQKRFSQGSVNPVLQRASSLLFDS IEDKKHATQRRAKGELFYGRR GTLTHFALQDLMCEMEGGAGCYLYPCGTAAVTNS ILSFVKTGDHVLMSGAAYEPTQYFCN IVLKKMQIDITYYDPLIGEDIATLIQPNTKVLFLEAPSSITMEI PDI PTIVKAARKVNPN IVIMIDNTWSAGVLFKALEHDIDIS IQAGTKYLVGHSDIMIGTAVANARTWDQLREHSYL MGQMVDADSAYTTARGIRTLGVRLKQHQESS I KVAKWLSEQPEVKTVYHPALP SCPGHEF FLRDFSGSSGLFSFELTQRLTSEQVSKFMDHFQLFAMAYSWGGFESLILCNQPEEIAHIR PNIKRNLTGSLIRVHIGFENVDELIADLKAGFERIA

$>$ POA 8 P1

MRLVQLSRHS IAFPSPEGALREPNGLLALGGDLSPARLLMAYQRGIFPWFSPGDPILWWS PDPRAVLWPESLHISRSMKRFHKRS PYRVTMNYAFGQVIEGCASDREEGTWITRGVVEAY HRLHELGHAHS IEVWREDELVGGMYGVAQGTLFCGESMFSRMENASKTALLVFCEEF IGH GGKLIDCQVLNDHTASLGACEIPRRDYLNYLNQMRLGRLPNNFWVPRCLFSPQE $>$ P0A821

MTTETRSLYSQLPAIDRLLRDSSFLSLRDTYGHTRVVELLRQMLDEAREVIRGSQTLPAW CENWAQEVDARLTKEAQSALRPVINLTGTVLHTNLGRALQAEAAVEAVAQAMRSPVTLEY DLDDAGRGHRDRALAQLLCRITGAEDACIVNNNAAAVLLMLAATASGKEVVVSRGELVEI GGAFRIPDVMRQAGCTLHEVGTTNRTHANDYRQAVNENTALLMKVHTSNYSIQGFTKAID EAELVALGKELDVPVVTDLGSGSLVDLSQYGLPKEPMPQELIAAGVSLVSFSGDKLLGGP QAGI IVGKKEMIARLQSHPLKRALRADKMTLAALEATLRLYLHPEALSEKLPTLRLLTRS AEVIQIQAQRLQAPLAAHYGAEFAVQVMPCLSQIGSGSLPVDRLPSAALTFTPHDGRGSH LESLAARWRELPVPVIGRIYDGRLWLDLRCLEDEQRFLEMLLK

$>$ P0A8P6

MTDLHTDVERYLRYLSVERQLSPITLLNYQRQLEAI INFASENGLQSWQQCDVTMVRNFA VRSRRKGLGAASLALRLSALRSFFDWLVSQNELKANPAKGVSAPKAPRHLPKNIDVDDMN RLLDIDINDPLAVRDRAMLEVMYGAGLRLSELVGLDI KHLDLESGEVWVMGKGSKERRLP IGRNAVAWIEHWLDLRDLFGSEDDALFLSKLGKRISARNVQKRFAEWGI KQGLNNHVHPH KLRHSFATHMLESSGDLRGVQELLGHANLSTTQIYTHLDFQHLASVYDAAHPRAKRGK $>Q 07739$

MMVHNYCAPENVTAPPHVSRVTQYNKRGIRMISKVTWRVAWESDLTNGDHAELSDFFKSV YGATGAFNALPFAGGRSWAGARPELRGIAYDESGVAAHMGVLRRFIKVGGEQIAVAELGL YGVRRDLEGLGIGHSTLAMLPVLKALGVPFAFGCFRNELRIHFQRFCRNGKGAIVDNVNI KSTQPDIYPDLPPTKIEKKAAVILPLTETLDRWPEGVDIERNGPEL

$>$ POA7D4

GNNVVVLGTQWGDEGKGKIVDLLTERAKYVVRYQGGHNAGHTLVINGEKTVLHLIPSGIL RENVTSI I GNGVVLSPAALMKEMKELEDRGI PVRERLLLSEACPLILDYHVALDNAREKA RGAKAIGTTGRGIGPAYEDKVARRGLRVGDLFDKETFAEKLKEVMEYHNFQLVNYYKAEA VDYQKVLDDTMAVADILTSMVVDVSDLLDQARQRGDFVMFEGAQGTLLDIDHGTYPYVTS SNTTAGGVATGSGLGPRYVDYVLGILKAYSTRVGAGPFPTELFDETGEFLCKQGNEFGAT TGRRRRTGWLDTVAVRRAVQLNSLSGFCLTKLDVLDGLKEVKLCVAYRMPDGREVTTTPL 
AADDWKGVEPIYETMPGWSESTFGVKDRSGLPQAALNYIKRIEELTGVPIDI ISTGPDRT ETMILRDPFDA

$>$ P 84308

TDTTNSIKHVISPLARQTLQDRDLTRPVAGKRPIRLLPWLQVVKIGGRVMDRGADAILPL VEELRKLLPEHRLLILTGAGVRARHVFSVGLDLGLPVGSLAPLAASEAGQNGHILAAMLA SEGVSYVEHPTVADQLAIHLSATRAVVGSAFPPYHHHEFPGSRI PPHRADTGAFLLADAF GAAGLTIVENVDGIYTADPNGPDRGQARFLPETSATDLAKSEGPLPVDRALLDVMATARH IERVQVVNGLVPGRLTAALRGEHVGTLIRTGVRPA

$>$ P69791

MMDLDNIPDTQTEAEELEEVVMGLI INSGQARSLAYAALKQAKQGDFAAAKAMMDQSRMA LNEAHLVQTKLIEGDAGEGKMKVSLVLVHAQDHLMTSMLARELITELIELHEKLKA $>$ P0A6V8

MTKYALVGDVGGTNARLALCDIASGEISQAKTYSGLDYPSLEAVIRVYLEEHKVEVKDGC IAIACP ITGDWVAMTNHTWAFS IAEMKKNLGFSHLEI INDFTAVSMA I PMLKKEHLIQFG GAEPVEGKPIAVYGAGTGLGVAHLVHVDKRWVSLPGEGGHVDFAPNSEEEAI I LEILRAE IGHVSAERVLSGPGLVNLYRAIVKADNRLPENLKPKDITERALADSCTDCRRALSLFCVI MGRFGGNLALNLGTFGGVFIAGGIVPRFLEFFKASGFRAAFEDKGRFKEYVHDI PVYLIV HDNPGLLGSGAHLRQTLGHIL

$>$ P26240

MSMFDKPFDYENGSKFEMGIWIGRQMAYGAFLGS I PFLLGLGLVLGSYGLGLMLPERAHQ APSPYTTEVVVQHATEVV

$>$ Q89UH1

MAKEELIQFEGLVTEILPDARYRVQLDAGHEIVAYTAGKMKKNRIKTLAGDRVTVEMSPY DLEKGRLIFRHKDERPATSGGPPRGGAQRGGQFRRR

$>$ POA9N4

SVIGRIHSFESCGTVDGPGIRFITFFQGCLMRCLYCHNRDTWDTHGGKEVTVEDLMKEVV TYRHFMNASGGGVTASGGEA ILQAEFVRDWFRACKKEGIHTCLDTNGFVRRYDPVIDELL EVTDLVMLDLKQMNDEI HQNLVGVSNHRTLEFAKYLANKNVKVWIRYVVVPGWSDDDDSA HRLGEFTRDMGNVEKIELLPYHELGKHKWVAMGEEYKLDGVKPPKKETMERVKGILEQYG HKVMF

$>$ Q7NRK9

MISKYNRPMENLSNLDYSSYLKGWIRTVPNWPQQGVMFRDITPLLQNPKTFRMLIDIYVH RYMMEKVDLVAGLDARGF I I GSVLAYELNVGFVPIRKKGKLPFTTVEEEYELEYGNAAVE MHTDACKPGDRVILIDDLVATGGTMMAGYKLLKRMGADVLEAAAI IELPELGGGQLVRDG GLDLFTVCSYEGH

$>Q 56415$

MLQSLNHLTLAVSDLQKSVTFWHELLGLTLHARWNTGAYLTCGDLWVCLSYDEARQYVPP QESDYTHYAFTVAEEDFEPLSQRLEQAGVTIWKQNKSEGASFYFLDPDGHKLELHVGSLA ARLAACREKPYAGMVFTSDEA

$>$ Q59685

MYLYIETLKQRLDAINQLRLERAFASMSDVFKQVYGLI PVLLHYHHPQLPGYIQGNVPHG TCFFEPDDVQRQWVNKLTNASCDEPMNGYTSGELPITGIYSMGSTSSIGQSHCSDIDIWV CHQSWLDQDERARLQRKCLLIEQWAGELGIDVTFFLIDENRFRHHASGSLGGEDCGSTQH ILLLDEFYRTAVRLAGKRLLWTMVPVEEEYHYDEYVNSLYAQGVLTPNEWLDLGGLGELS AEEYFGASLWQLYKSVDSPYKAVLKS ILLEAYSADYPNGKLLALEMKQHLHRGEIVNYGL DAYCMMLERVTRYLVS INDLTRLDLIRRCFYLKVCEKLSNEKNENEPAGWRRQVLSQLVT QWQWDHERLAILDNRDSWKIERVRNAHNELLDTMMQSYRNLIRFARRNNLSVSASPQDIG VLTRKLYAAFEALPGKVTLVNPQISPDLSEPHLTFIYVPPGRANRSGWYLYNRAPDFAHI VGHQPLEYNRYLNKLVAWSYFNGLLTKDSQVY IHQGDSSCDEIKLHELVRDISSHFPIRL PAPTPKALYSPCEIRHLAI IVNLEVDPTERFSDQVVHFDFRKLDVFSFGEEEQCLIGSID LLYRNSWNEVRTLHFNGTQSMLESLKTILGKMHQDAAPPASVEVFCYSQHLRGLIRTRVQ QLVSECIELRLSTNRLEPGRFKALRIAGQTWGLFFERLNVSVQKLENAIEFYGAISYNKL HGLPVKLGKDARYLPAVIDGFACEGI IQFFFETTEDNNVFNIY I LDEANRVEIYSHCEGS KEELVKDVSRFYSSSHDRFTYGSSFINFNLPQFYQIVKVDGATQVLPFAGGSFGKLSDLG KTAPKEEMSTKPIQGFNDYQAVHHH

$>$ Q8XYN6

MAHFSCFPGALALSAFRQQRLLSTLKRIDPEIDAVSAQYLHFVAADAPLSADDAARVQAL LTYGSPASAETEGDRFVVIPRFGTISPWASKATEIARHCALPQI HRIERGVEFTVTCKKG LLRGLTGGRKQLDEATRAAVAAHLHDRMTEIVVATREAGYGLFDVLPAKALRFVDLGSGD 
AAAGRGALEAANTEMGLALSDDEIDYLVDAYRKLGRNPTDVELMMFAQANSEHCRHKIFN ADWTIDGETQDKSLFAMIRNTHQLAPQGTVVAYSDNAAVMEGGMAERWFPHAGTDGETGV PQYGRREALTHTLMKVETHNHPTAISPFPGASTGAGGEIRDEGATGRGAKPKAGLTGFTV SNLLLPEAVQSWENARDTAQPAAQRNPGDTAPGPVGKPDRIASPLQIMIEGPIGGAAFNN EFGRPNLGGYFRVYEQNVGGTVRGYHKPIMIAGGI GNIDAGHTHKHGLPAGTLLVQLGGP GMRIGMGGGAASSMATGTNTADLDFDSVQRGNPEMQRRAQEVINACWALGEDNPILS IHD VGAGGISNAFPELVDGADRGARFDLRQVHLEESGLSPAEIWCNESQERYTLAIAPGDFPR FQAMCERERAPFSVVGFATEEQQLQVVDGDAPADAVEHFPVNMPMDVLLGKPPRMHRDVR RVAQALPEVDVTGLDLETVARDVLRHPTVASKSFLITIGDRTVGGLNTRDQMVGPWQVPV ADVAVTTLDYKGYAGEAMTMGERTPLAVIDAPASGRMAIGEAITNIAAAPIASLAQLKLS ANWMAACGVDGEDARLYDTVKAVGMELCPALGIS I PVGKDSLSMRTKWDDAGEAKEVVAP VSLIVSAFAPVTDVRKTLTPQLKPVASAGEAADTTLIVIDLGHGKHRLGGS ILAQVTQQI GNSVPDVGDAEDLKRFFAAIQQLNAAGMLLAYHDRSDGGLWATVCEMAFAGHCGVS INVD MLTLDGAHASDYGDAKNWAQQVSGRRADMTLRALFAEELGAVIQVPAAQRDAVFAVLREH GLAACSHVIGAPNASGQIEIWRDAKKVFSAPR IELQRAWTDVSWRIASLRDNPECTQSEY DRLLDAEDPGISPNLTFDLAEDVAAPFIATGARPRMAILREQGVNSQVEMAYAMDKAGFD AYDVHMSDLLAGRTRLADFKGFVACGGFSYGDVLGAGEGWAKTILFNGMLAEQFAAFFNR ADS IALGVCNGCQMMANLAP I I PGAGAWPKFTRNQSEQYEGRLVTVQVEASPS I FYAGME GSRI P IVVAHGEGYADF SQQGDIGKVAVGLRYVDNRGEVTQTYPLNPNGSPQGIASVTTH DGRFTVLMPHPERVFRAVQMSWHPKDWAAAGDGSSPWMRMFRNARKQMG

$>$ P32169

MQNITQSWFVQGMIKATTDAWLKGWDERNGGNLTLRLDDADIAPYHDNFHQQPRYIPLSQ PMPLLANTPF IVTGSGKFFRNVQLDPAANLGIVKVDSDGAGYHILWGLTNEAVPTSELPA HFLSHCERIKATNGKDRVIMHCHATNLIALTYVLENDTAVFTRQLWEGSTECLVVFPDGV GILPWMVPGTDEIGQATAQEMQKHSLVLWPFHGVFGSGPTLDETFGLIDTAEKSAQVLVK VYSMGGMKQTISREELIALGKRFGVTPLASALAL

$>068703$

MNS IHGHYHIQLSNYSAGENLQSATLTEGVIGAHRVKVETALSHSNLQKKLSATIKHNQS GRSMLDRKLTSDGKANQRSSFTF SMIMYRMIHFVLSTRVPAVRESVANYGGNINFKFAQT KGAFLHKI I KHSDTASGVCEALCAHWIRSHAQGQSLFDQLYVGGRKGKFQIDTLYS IKQL QIDGCKADVDQDEVTLDWFKKNGISERMIERHCLLRPVDVTGTTESEGLDQLLNAILDTH GIGYGYKKIHLSGQMSAHAIAAYVNEKSGVTFFDPNFGEFHFSDKEKFRKWFTNSFWGNS MYHYPLGVGQRFRVLTFDSKEV

$>083004$

MNPSTLPAPPLAEVYDVAVIGGGINGVGIAADAAGRGLSVFLCEKDDLASHTSSASSKLI HGGLRYLEHYEFRLVREALAEREVLLAKAPHIVKQMRFVLPHRPHLRPAWMIRAGLFLYD HLGKREKLAGSKSLKFGANSPLKSEITKGFEYSDCWVDDARLVVLNAMAAREKGAHIHTQ TRCISAHRSNGLWEMNMERADGSLFS IRARALVNAAGPWVAKF IRDDLKLDSPYGIRLIQ GSHLIVPRLYEGAHAHILQNEDQR IVFTI PYLNHLTI IGTTDREYTGDPAKVAITEGETD YMLKVVNAHFKKQLSRDDIVHTYSGVRPLCNDESDNPSAITRDYTLALSGGNGEAPILSV FGGKLTTYRKLAESAMAQLAPFFTQMRPSWTAKASLPGGENMTTPERAGRRHPRQIRLGT ERDAPRRWATTYGSRTWRLLEGVQALADLGDHLGGGLYTREVDYLCAEEWATQPQDILWR RTKLGLFTTAEEQDNVQRYLSKVGQTRAKIEAA

$>$ Q92YM4

MVRILLATSTVELEDLVKRAIEGDASAELVLIARSGREAVRMTGELLPDIVAVELCPSGD DSAETVREIMIAAPTPVVMLSHRDGSQLGTISARALEAGALAVI PAPAAHGMQLEQPAIE KFLSTIKAMSQVKVVRQWRQKVRGDRAAKDQPPTARTPIGIVGIAASTGGPAAIRAILKD I SADLPVPILIVQHMSNGF IDGVAASLNATVPLTVKVARNGELLKPGTVYLAPDNCQLGV SGRSRLRVSDDAPVNGFKPSGSYLFGS IARAFKGESLAVVLTGMGDDGTEGLRALRMAGG KAIAQDEKSSVVFGMPKSAIGAGLVDLVLPLESIAENITAIARGRSEPEG

$>$ Q9APM5

MTYDKAELVALDKKYVWHHLTQHKNFEPAIYVKGEGMRITDIDGKTYLDAVSGGVWTVNV GYGRKEIVDAVAKQMMEMCYFANGIGNVPTI KFSEKLISKMPGMSRVYLSNSGSEANEKA FKIVRQIGQLKHGGKKTGILYRARDYHGTTIGTLSACGQFERKVQYGPFAPGFYEFPDCD VYRSKFGDCADLGVKMAKQLEEVILTVGPDELGAVIVEPMTAGGGILVPPAGYYETIREI CDKYELLLI IDEVVCGLGRTGKWFGYQHFNVQPDIVTMAKGVASGYAPISCTVTTEKVFQ DFVNDPADTDAYFRDISTFGGCTSGPAAALANIEI IERENLLENCTKMGDRLLEGLKGLM AKHP I IGDVRGKGLFAGIEIVKDRATKEP IAEAVANAMVGAAKQAGVLIGKTSRSFREFN NTLTLCPALIATEADIDEIVAGIDKAFTTVEQKFGL 
$>$ P33547

MCRKLYDKLYEITGAKLDFNDKNQAF ILLEEQI PVCITDNDEY IFLTGLLNEHELFTENI INPEHILILNYSLSRDYGSS ICLLPDTHQCVLTKKHYKKYLSPDELIESLYEFLFCIKLT IANITSEVN

$>$ POA1W4

MKNIRNFS I IAHIDHGKSTLSDRI IQICGGLSDREMEAQVLDSMDLERERGITIKAQSVT LDFKASDGETYQLNFIDTPGHVDFSYEVSRSLAACEGALLVVDAGQGVEAQTLANCYTAM EMDLEVVPVLNKIDLPAADPERVAEEIEDIVGIDATDAVRCSAKTGVGVTDVLERLVRDI PPPQGDPDGPLQALI IDSWFDNYLGVVSLVRI KNGTMRKGDKI KVMSTGQTYNADRLGIF TPKQVDRTELKCGEVGWLVCAI KDILGAPVGDTLTSARNPAEKALPGFKKVKPQVYAGLF PVSSDDYESFRDALGKLSLNDASLFYEPESSSALGFGFRCGFLGLLHMEI IQERLEREYD LDLITTAPTVVYEVETTAKETIYVDSPSKLPPLNNIYELREPIAECHMLLPQAYLGNVIT LCIEKRGVQTNMVYHGNQVALTYEI PMAEVVLDFFDRLKSTSRGYASLDYNFKRFQASDM VRVDVLINNERVDALALITHRDNSQSRGRELVEKMKDL I PRQQFDIAIQAAIGTHI IARS TVKQLRKNVLAKCYGGDISRKKKLLQKQKEGKKRMKQ I GNVELPQEAFLAILHVGKDNK $>$ P21244 MSSRSELLLDRFAEKIGVGS ISFNENRLCSFAIDEIYYISLSDANDEYMMIYGVCGKFPT DNPNFALEILNANLWFAENGGPYLCYESGAQSLLLALRFPLDDATPEKLENEIEVVVKSM ENLYLVLHNQGITLENEHMKIEEISSSDNKHYYAGR

$>$ P30139

MLRIADKTFDSHLFTGTGKFASSQLMVEAIRASGSQLVTLAMKRVDLRQHNDAILEPLIA AGVTLLPNTSGAKTAEEAI FAAHLAREALGTNWLKLE I HPDARWLLPDPIETLKAAETLV QQGFVVLPYCGADPVLCKRLEEVGCAAVMPLGAPIGSNQGLETRAMLEI I IQQATVPVVV DAGIGVPSHAAQALEMGADAVLVNTAIAVADDPVNMAKAFRLAVEAGLLARQSGPGSRSY FAHATSPLTGFLEASA

$>$ P51837

MNHLNKLMERLGHQFNNLELLKIALTHCSSGADNNERLEFLGDSVLGF I IASELYQRRPQ AREGDLSRMRASMVNGDELAQMSTKLGINEYLQLGVGEQKSGGKRRRS I LADALEA IVGA IYIDAGLETCRRCVLNWYGERVDDLSKLSPKKDAKSLLQEWLQARRLPLPTYEVKITGEA HAQTFTVNCYVKGLPHKTEGVNTTRRRAEQIAAKRFLELLDDGKGDGITERDQ

$>$ Q3 JSY9

MASNNVAQFAAELKMPAGVLLEQLQAAGVQKASEDDALSETDKARLLDHLRKSHGATDGD KRKITLTRRHTSEIKQADATGKARTIQVEVRKKRTFVKRDDVSETGADQAQAQTDEQAEA ELKRREEEARREAELLEKQAQELRERQERLEREEAERRAREEAAEAERRRAEEEAAAKRA AAAQAEAAQQAAAAREQAQRAQSEPAEQSAQDEARAAAERAAQREAAKKAEDAAREAADK ARAEQEEIRKRREAAEAEARAIREMMNTPRRAQVKAVEPPKPAEPPAAKAAEAKGTLHKP AKPAGEAAAARPAAKKPASGAPAPAAAPAGDRTKKPGTGKSGWQDDAAKRRGIKTRGDSS GGVDRGWRGGPKGRGKHQDSASSFQAPTEPIVREVHVPETISVADLAHKMS I KASEVI KV MMKMGQMVTINQVLDQETAMIVVEELGHRALAAKLDDPEALLVEGEIGSDAEQLPRPPVV TVMGHVDHGKTSLLDYIRRAKVAAGEAGGITQHIGAYHVETPRGVVTFLDTPGHEAFTAM RARGAKATDIVILVVAADDGVMPQTKEAISHAKAGGVPIVVAINKIDKPEANPDRVKQEL VAEGVVPEEYGGDSPFVPVSAKTGAGIDDLLENVLLQAEVLELKAPVESPAKGIVIEAKL DKGKGPVATVLVQSGTLSRGDVVLAGTAYGRVRAMLDENGKPTKEAGPS I PVEIQGLSEV PGAGEEVIVLPDERKAREIALFRQGKFRDVKLAKQQAAKLESMLEQMGEGEVQNLPLI IK ADVQGSQEALVQSLLKLSTDEVRVQIVHSAVGGISESDVNLATASKAVI I GFNTRADAQA RKLAEANGIDIRYYNI IYDAVDEVKAAMSGMLAPEKREVVTGMVEVRQVFKVPKVGTVAG CMVTDGVVKRSSSVRVLRNNVVI FTGELDSLKRFKDDVKEVKQGFECGMSLKNFNDIVEG DQFEVFEVTEVARTL

$>$ Q885R6

MKTGKELKPGTVIRLENDPWLVQKAEFTKSGRNSAIMKTKLKNLLTGYKTEIVYSADDKL DDVILDRKEATLSFISGDTYTFMDTTDYTMYELNAEDIESVLPFVEEGMTDVCEAVFFDE RLVSVELPTTIVRQVDYTEGSARGDTSGKVMKPAKLKNGTELSVADFIEIGDMIEIDTRE GGSYKGRAK

$>$ POABD5

SLNFLDFEQPIAELEAKIDSLTAVSRQDEKLDINIDEEVHRLREKSVELTRKIFADLGAW QIAQLARHPQRPYTLDYVRLAFDEFDELAGDRAYADDKAIVGGIARLDGRPVMI I GHQKG RETKEKIRRNFGMPAPEGYRKALRLMQMAERFKMPI ITFIDTPGAYPGVGAEERGQSEAI ARNLREMSRLGVPVVCTVIGEGGSGGALAIGVGDKVNMLQYSTYSVISPEGCAS I LWKSA DKAPLAAEAMG I IAPRLKELKL IDS I I PEPLGGAHRNPEAMAASLKAQLLADLADLDVLS 
TEDLKNRRYQRLMSYGYA

$>$ P32173

MNLMTTITGVVLAGGKARRMGGVDKGLLELNGKPLWQHVADALMTQLSHVVVNANRHQEI YQASGLKVIEDSLADYPGPLAGMLSVMQQEAGEWFLFCPCDTPYIPPDLAARLNHQRKDA PVVWVHDGERDHPTIALVNRAIEPLLLEYLQAGERRVMVFMRLAGGHAVDFSDHKDAFVN VNTPEELARWQEKR

$>$ Q62LE0

MSASDLTSVQAGAPQGRRQILVTSALPYANGQIHIGHLVEYIQTDIWVRTMRMHGHEIYY IGADDTHGTPVMLRAEQEGVSPKQLIERVWREHKRDFDSFGVSFDNFYTTDSDENRVLSE TIYLALKEAGF IAEREIEQAYDPVRQMFLPDRF I KGECPKCHAKDQYGD SCEVCGTTYQP TDLIHPYSVVSGAAPVRKTSTHYFFRLSDPRCEAFLREWVSGLAQPEATNKMREWLGEAG EAKLADWDI SRDAPYFGFEI PGAPGKYFYVWLDAPVGYYASFKNLCQRRGLDFDAWIRKD STTEQYHF IGKDILYFHTLFWPAMLEFSGHRTPTNVFAHGFLTVDGAKMSKSRGTFITAQ SYIDTGLNPEWLRYYFAAKLNATMEDIDLNLEDFQARVNSDLVGKYVNIASRAAGFLLKR FDGRVQASAMNHPLLATLRGAI PQIAAHYEAREYGRALRQTMELADAVNGYVDSAKPWEL AKDPANAVALHETCSVSLEAFRLLSLALKPVLPRVAQGVEAFLGIAPLTWADAGTPLSPE QPVRAYQHLMTRVDPKQIDALLAANRGSLQGTAAAAEAGAANGNGAGSKNGKGAKAAAQP AASAANADDGASPI I S IDDFAKIDLRIAKIVACQAVEGSDKLLQLTLDVGEERTRNVFSG IKSAYRPEQLVGKLTVMVANLAPRKMKFGLSEGMVLAASAADEKAEPGLYILEPHSGAKP GMRVK

$>$ Q8GHL1

MTALYWQTIGEGERDLVLLHGWGLNAEVWSCIQALTPHFRLHLVDLPGYGRSQGFGALSL AQMTEIVLAAAPPQAWWLGWSLGGLVASQAALMQPQRVSGLITVASSPCFAARDEWPGIR PDVLSGFQHQLSLDFQRTVERFLALQTLGTESARQDARQLKAVVLNQPTPSVEVLNGGLE ILRTADLRAPLAELNLPLLRIYGYLDGLVPRKVAELLDAAWPNSTSQIVAKAAHAPF ISH PDEFVTMIEAFIAAH

$>$ P0A6I 6

MQKRAIYPGTFDP ITNGHIDIVTRATQMFDHVILAIAASPSKKPMFTLEERVALAQQATA HLGNVEVVGFSDLMANFARNQHATVLIRGLRAVADFEYEMQLAHMNRHLMPELESVFLMP SKEWSFISSSLVKEVARHQGDVTHFLPENVHQALMAKLA

$>$ P04772

MTKYKLEYIWLDGYTPTPNLRGKTQIKEFASFPTLEQLPLWGFDGSSTQQAEGHSSDCVL KPVAVFPDAARTNGVLVMCEVMMPDGKTPHASNKRATILDDAGAWFGFEQEYFFYKDGRP LGFPTSGYPAPQGPYYTGVGFSNVGDVARKIVEEHLDLCLAAGINHEGINAEVAKGQWEF QIFGKGSKKAADEMWMARYLMLRLTEKYGIDIEFHCKPLGDTDWNGSGMHANFSTEYMRT VGGKEYFEALMAAFDKNLMDHIAVYGPDNDKRLTGKHETAPWNKFSYGVADRGAS IRVPH SFVNNGYKGYLEDRRPNSQGDPYQIASQILKTISSVPTEKKAVA

$>$ P08622

AKQDYYEILGVSKTAEEREIRKAYKRLAMKYHPDRNQGDKEAEAKFKEIKEAYEVLTDSQ KRAAYDQYGHAAFEQGGMGGGGFGGGADFSDI FGDVFGDI FGGGRGRQRAARGADLRYNM ELTLEEAVRGVTKEIRI PTLEECDVCHGSGAKPGTQPQTCPTCHGSGQVQMRQGFFAVQQ TCPHCQGRGTLIKDPCNKCHGHGRVERSKTLSVKI PAGVDTGDRIRLAGEGEAGEHGAPA GDLYVQVQVKQHPIFEREGNNLYCEVPINFAMAALGGEIEVPTLDGRVKLKVPGETQTGK LFRMRGKGVKSVRGGAQGDLLCRVVVETPVGLNERQKQLLQELQESFGGPTGEHNSPRSK SFFDGVKKFFDDLTR

$>$ POA2U4

MSLNITENES ISTAVIDAINSGATLKDINAI PDDMMDDIYSYAYDFYNKGRIEEAEVFFR FLCIYDFYNVDY IMGLAAIYQI KEQFQQAADLYAVAFALGKNDYTPVFHTGQCQLRLKAP LKAKECFELVIQHSNDEKLKIKAQSYLDAIQDIKE

$>$ P37330

SQTITQSRLRIDANFKRFVDEEVLPGTGLDAAAFWRNFDEIVHDLAPENRQLLAERDRIQ AALDEWHRSNPGPVKDKAAYKSFLRELGYLVPQPERVTVETTGIDSEITSQAGPQLVVPA MNARYALNAANARWGSLYDALYGSD I I PQEGAMVSGYDPQRGEQVIAWVRRFLDESLPLE NGSYQDVVAFKVVDKQLRIQLKNGKETTLRTPAQFVGYRGDAAAPTCILLKNNGLHIELQ IDANGRIGKDDPAHINDVIVEAA IST ILDCEDSVAAVDAEDKI LLYRNLLGLMQGTLQEK MEKNGRQIVRKLNDDRHYTAADGSE I SLHGRSLLF IRNVGHLMT I PVIWDSEGNE I PEGI LDGVMTGAIALYDLKVQKNSRTGSVYIVKPKMHGPQEVAFANKLFTRIETMLGMAPNTLK MGIMDEERRTSLNLRSCIAQARNRVAFINTGFLDRTGDEMHSVMEAGPMLRKNQMKSTPW IKAYERNNVLSGLFCGLRGKAQIGKGMWAMPDLMADMYSQKGDQLRAGANTAWVPSPTAA 
TLHALHYHQTNVQSVQANIAQTEFNAEFEPLLDDLLTI PVAENANWSAQEIQQELDNNVQ GILGYVVRWVEQGIGCSKVPDI HNVALMEDRATLRISSQHIANWLRHGILTKEQVQASLE NMAKVVDQQNAGDPAYRPMAGNFANSCAFKAASDLIFLGVKQPNGYTEPLLHAWRLREKE $\mathrm{SH}$

\section{$>$ Q9JW39}

MQEQYQPAAIEPAAQKKWDDARIFNVSEDASKPKYYCLSMFPYPSGKLHMGHVRNYTIGD VLSRFKLLNGFNVMQPMGWDAFGMPAENAAMKNNVAPAAWTYDNIEYMKTQLKSLGFAID WARETATCKPEYYRWEQWLFTKLFEKGIVYRKNGTVNWDPVDQTVLANEQVIDGRGWRSG ALIEKREI PMYYFKITDYAEELLNDLDKLEHWPEQVKTMQRNWIGKSRGMTVRFAVSDDS KQGLEGDYAKFLQVYTTRPDTLMGATYVAVAAEHPLATAAAADKPELQAF IAECKAGSVA EADMATMEKKGVPTGRYVVNPLNGDKLEVWIANYVLWGYGDGAVMAVPAHDERDFEFAAK YSLPKKQVLQRVTIGLSPEQEEAMGFGGGSGCGAGYGDGSGSDSRDLPFMPNKWQAWYGV KDDFMVLINSGELDGMNYQTAFDAVAAKLQAIGAGEPKTQYRLRDWGISRQRYWGCPI PI VHCEQCGDVPVPADQLPVVLPENVVPDGMGSPLAKMPEFYETTCPCCGGAAKRETDTMDT FMESSWYFFRYMSPKFSDGMVDPAAAKYWGAVDQYIGGIEHAILHLLYARFFTKLMRDEG LVNVDEPFERLLTQGMVVCETYYRENDKGGKDWINPADVELTFDDKGRPVSAVLKVDGLP VVISGTEKMSKSKNNGVDPQELINAYGADTARLFMMFAAPPEQSLEWSDSGVEGAHRFLR RLWRTVYEYLKQGEAVNAFAGSQDGLSKELKDLRHKLHSTIAKVSDDYGRRQQFNTAIAA VMELLNQYDKTDTGSEQGRTVAQEVLEAAVRLLWPIVPHICETLWSELNGAKLWEAGWPA VDEAALVKSEIEVMVQVNGKLRGKITVAADASKADLEAAALATEGAVKFMEGKPAKKI IV VPGRLVNIVV

$>$ P23869

MVTFHTNHGDIVIKTFDDKAPETVKNFLDYCREGFYNNTI FHRVINGFMIQGGGFEPGMK QKATKEP I KNEANNGLKNTRGTLAMARTQAPHSATAQFF INVVDNDFLNF SGESLQGWGY CVFAEVVDGMDVVDKI KGVATGRSGMHQDVPKEDVIIESVTVSE

$>$ P60595 MNVVILDTGCANLNSVKSAIARHGYEPKVSRDPDVVLLADKLFLPGVGTAQAAMDQVRER ELFDLIKACTQPVLGICLGMQLLGRRSEESNGVDLLGI IDEDVPKMTDFGLPLPHMGWNR VYPQAGNRLFQGIEDGAYFYFVHSYAMPVNPWTIAQCNYGEPFTAAVQKDNFYGVQFHPE RSGAAGAKLLKNFLEM

\section{$>$ P75949}

MGPVMLDVEGYELDAEEREILAHPLVGGLILFTRNYHDPAQLRELVRQIRAASRNRLVVA VDQEGGRVQRFREGFTRLPAAQSFAALSGMEEGGKLAQEAGWLMASEMIAMDIDISFAPV LDVGHISAA I GERSYHADPQKALAIASRF IDGMHEAGMKTTGKHFPGHGAVTADSHKETP CDPRPQAEIRAKDMSVFSSLIRENKLDAIMPAHVIYSDVDPRPASGSPYWLKTVLRQELG FDGVIFSDDLSMEGAAIMGSYAERGQASLDAGCDMILVCNNRKGAVSVLDNLSPIKAERV TRLYHKGSFSRQELMDSARWKAISTRLNQLHERWQEEKAGH

$>$ P39440

MTQHSPYSSAMAEQRHQEWLRFVELLRQSYDKDLHLPLLQLMLTPDEREALGTRVRI IEE LLRGEMSQRELKNELGAGIATITRGSNSLKSAPVELRQWLEQILLGAQR

$>$ Q9ABY6

MFEGLSPLARDARSWPFEQARATIARVLRVRLPDRADQDAAKALIDAGKTDEAVKAYPAL AKAVIFETGYGPSGLPHLGTFGEVARTTMVRQAFRALTDEAIPTRLIAFSDDMDGLRKVP DNIENKQPLIEDLGKPLTVVRDPFGTHDSFGAHNNARLRAFLDGFGFEYEFVSSTDCYKG GLFDATLLTALERFDAIQKVMLPTLGEERRATYSPFLPISPSTGKVLQVPTLERNVEKGT IVFEDEDGSKVEVPVTGGHVKMQWKPDWAMRWTALGVDYEMSGKDLIDSVKASGAICKAL GGVPPEGFNYELFLDENNQKISKSKGNGLSMEDWLRYGAPESLSYYMFQSPKSAKKLYFD VIPKASDEYLQQLDGFGRQEPAKQLDNPVWHIHGGKPPQQGSPVSFSLMLNLVSAADAST KEILWGFLSRYI PGASPETQPLLDRLAGYAINYYEDFVKPSKVFRAPSDQERAAMLDLLA KLKAMPAGTQDAELIQNEVFEVGKTHGFDPLRAWFQALYEVLLGQSQGPRFGSFAAIFGI DRTVALIEEK

$>$ P0A9A6

MFEPMELTNDAVIKVIGVGGGGGNAVEHMVRERIEGVEFFAVNTDAQALRKTAVGQTIQI GSGITKGLGAGANPEVGRNAADEDRDALRAALEGADMVF IAAGMGGGTGTGAAPVVAEVA KDLGILTVAVVTKPFNFEGKKRMAFAEQGITELSKHVDSLITI PNDKLLKVLGRGISLLD AFGAANDVLKGAVQGIAELITRPGLMNVDFADVRTVMSEMGYAMMGSGVASGEDRAEEAA EMAISSPLLEDIDLSGARGVLVNITAGFDLRLDEFETVGNTIRAFASDNATVVIGTSLDP DMNDELRVTVVATGIGMDKRPEITLVTNKQVQQPVMDRYQQHGMAPLTQEQKPVAKVVND NAPQTAKEPDYLDI PAFLRKQAD 
$>$ P12295

ANELTWHDVLAEEKQQPYFLNTLQTVASERQSGVTIYPPQKDVFNAFRFTELGDVKVVIL GQDPYHGPGQAHGLAFSVRPGIAIPPSLLNMYKELENTI PGFTRPNHGYLESWARQGVLL LNTVLTVRAGQAHSHASLGWETFTDKVI SLINQHREGVVFLLWGSHAQKKGAI IDKQRHH VLKAPHPSPLSAHRGFFGCNHFVLANQWLEQRGETPIDWMPVLPAESE

$>$ P0A6Y5

MPQHDQLHRYLFENFAVRGELVTVSETLQQILENHDYPQPVKNVLAELLVATSLLTATLK FDGDITVQLQGDGPMNLAVINGNNNQQMRGVARVQGEI PENADLKTLVGNGYVVITITPS EGERYQGVVGLEGDTLAACLEDYFMRSEQLPTRLF IRTGDVDGKPAAGGMLLQVMPAQNA QQDDFDHLATLTETIKTEELLTLPANEVLWRLYHEEEVTVYDPQDVEFKCTCSRERCADA LKTLPDEEVDS ILAEDGEIDMHCDYCGNHYLFNAMDIAEIRNNASPADPQVH

$>$ P21762

MLVTKLAPDFKAPAVLGNNEVDEHFELSKNLGKNGA I LFFWPKDFTFVCPTEI IAFDKRV KDFQEKGFNVIGVSIDSEQVHFAWKNTPVEKGGIGQVTFPMVADITKS ISRDYDVLFEEA IALRGAFLIDKNMKVRHAVINDLPLGRNADEMLRMVDALLHFEEHGEVCPAGWRKGDKGM KATHQGVAEYLKENSIKL

$>$ P08997

MTEQATTTDELAFTRPYGEQEKQILTAEAVEFLTELVTHFTPQRNKLLAARIQQQQDIDN GTLPDFISETAS IRDADWKIRGI PADLEDRRVEITGPVERKMVINALNANVKVFMADFED SLAPDWNKVIDGQINLRDAVNGTISYTNEAGKIYQLKPNPAVLICRVRGLHLPEKHVTWR GEAI PGSLFDFALYFFHNYQALLAKGSGPYFYLPKTQSWQEAAWWSEVFSYAEDRFNLPR GTIKATLLIETLPAVFQMDEILHALRDHIVGLNCGRWDYIFSYIKTLKNYPDRVLPDRQA VTMDKPFLNAYSRLLIKTCHKRGAFAMGGMAAF I PSKDEEHNNQVLNKVKADKSLEANNG HDGTWIAHPGLADTAMAVFNDI LGSRKNQLEVMREQDAP ITADQLLAPCDGERTEEGMRA NIRVAVQYIEAWISGNGCVPIYGLMEDAATAEISRTS IWQWIHHQKTLSNGKPVTKALFR QMLGEEMKVIASELGEERFSQGRFDDAARLMEQITTSDELIDFLTLPGYRLLA $>$ P0A2 82

MAIKLIVGLANPGAEYAATRHNAGAWYVDLLAERLRAPLREEPKFFGYTSRITLEGEDVR LLVPTTFMNLSGKAVGAMASFYRIQPDEILVAHDELDLPPGVAKFKLGGGHGGHNGLKDI ISKLGNNPNFHRLRVGIGHPGDKNKVVGFVLGKP PVSEQKLIDEAIDEAARCTELWFKEG LAKATSRLHTFKAQ

$>$ POA1Y2

MSIDIDI I KARAKNEYRLSKVRGEAMISVRI PGGILPAHLLTVARDIAETWGNGQIHLTT RQKLAMPGIRYEDIDNVNAALEPFLREIEIELCDVQVEDTKAGYLAIGGRNIVACQGNRI CQKANTDTTGLSRRLEKLVYPS PYHLKTVIVGCPNDCAKASMADLGI IGVAKMRFTADRC IGCGACVKACSHHAVGCLALKNGKAVKEESACIGCGECVLACPTLAWQRKPDQLWQVRLG GRTSKKTPRVGKLFLNWVTEDVIKQVIVNLYEFEKEMLGGKP IYLHMGHLIDKGGYLRFK ERVLRGVQLNPEAMVAERIYWAEDESVARMHLKPAGH

$>$ P45363

MSDTIVIVDAGRTAIGTFGGALSALQATDIGTTVLKALIERTGIAPEQVSEVILGQVLTA GCGQNPARQTTLMAGLPHTVPAMTINKVCGSGLKAVHLAMQAVACGDAEIVIAGGQESMS QSSHVLPRSREGQRMGDWPMKDTMIVDGLWDAFNQCHMGVTAENIAKKYAFTREAQDAFA AASQQKAEAAIQSGRFADEI I PVS I PQRKGDPLVFDTDEFPRPGTTAETLGRLRPAFDKQ GTVTAGNASGINDGAAMVVVMKESKAKELGLTPMARLVAFSSAGVDPAIMGTGP I PASTD CLKKAGWAPADLDLVEANEAFAAQAMSVNQEMGWDLSKVNVNGGAIAIGHPIGASGARVL VTLLYEMQKRDAKKGLATLCIGGGQGVALAVERL

$>$ P 81433

MELKNHKKI I ILGSGPAGYTAA IYSSRANLNPLLITGINKGGQLMNTNEIENWPGDFKKI TGPELMNRMHEHSLKFKTEIVYDNI I SVEFKKKPFFLLGEYNKYTCDAVI IATGANPRYL GLSSENKFKGKGISTCAVCDGFFYKNKEIAVVGGGNTAIEETLYLSNFVKKIYLIHRRNN FKAEKILIDRLLKIVKTKKVILHLNSTIEDILGNNKGVTHLLI KNKNLKEKKKLKIAVSG LFVAIGYI PNTDIFTDQLKMKDGYIKI KKGTHGNYTQTNI PGVFAAGDVIDHVYRQAITS SASGCMAALDSERYLNSLS

$>$ P43836

MTDINTVLAELKRGTDEILSEADLIEKLKENRPLKVKLGADPTAPDIHLGHTVVLNKLRQ FQQLGHEVYFLIGDFTGMVGDPSGKNATRPPLSREDVLRNAETYKEQIYKILDPQKTKIV FNSEWLSKLGTXGMIRLASNYTVARMLERDDFKKRFGNNQP IA I HEF IYPLLQGYDSVAL DADVELGGTDQKFNLLVGRELQKSAGKKPQVAITLPLLVGLDGEKKMSKSLGNYIGVTEA PSDMFGKVMS ISDELMWDWYNLLSFRPLNEIAQLKSEVENGKNPRDVKILLAKELIARFH 
NEEAANAAEQEFINRFQKGAMPDEMPEFTFSGEMGLATLLKEAGLVPSTSEAIRSAQQGG VKINGEKVDNVKDNAPKGTNVYQVGKRKFARVRLNKVDTV

$>$ P0A6K1

MQFSKMHGLGNDFMVVDAVTQNVFFSPELIRRLADRHLGVGFDQLLVVEPPYDPELDFHY RIFNADGSEVAQCGNGARCFARFVRLKGLTNKRDIRVSTANGRMVLTVTDDDLVRVNMGE PNFEPSAVPFRANKAEKTYIMRAAEQTILCGVVSMGNPHCVIQVDDVDTAAVETLGPVLE SHERFPERANIGFMQVVKREHIRLRVYERGAGETQACGSGACAAVAVGIQQGLLAEEVRV ELPGGRLDIAWKGPGHPLYMTGPAVHVYDGFIHL

$>$ Q84 H4 I

AATDNRKVVEGVHKMTPSEAFVETCVANGVSEMFGIMGSAFMDAMDIFAPAGIRLI PVVH EQGAAHMADGYARVSGRHGVVIGQNGPGI SNCVTGIAAAYWAHSPVVIVTPETGTMGMGL GGFQEANQLPMFQEFTKYQGHVCNPKRMAEFTGRVFDRAMSEMGPTQLNI PRDYFYGEIE CEIPKPMRVDRGHGGEASLQAAVELLKTAKFPVILAGGGVVMGDAVEEAKQLAERLGAPV ATGYLRNDAFPAKHPLWAGPLGYQGSKAAMKLIAQADVVIALGSRMGPFGTLPQHGMDYW PKAAKI IQIEADHTNLGLVKKIAVGINGDAKAVAAELSRRLADVTLGCDATKAARADTIA TEKAAWEKELDGWTHERDPYSLDMIEEAKGERTPTGGSYLHPRQVLRELEKAMPARVMVS TDIGNINSVANSYLRFDEPRSFFAPMSFGNCGYALPTI IGAKCAAPDRPAIAYAGDGAWG MSMMEIMTAVRHDI PVTAVVFHNRQWGAEKKNQVDFYNRRFVAGELESESFSDIAKAMGA EGIVVDHIEDVGPALQKAIDMQMKEGKTCVIEIMCTRELGDPFRRDALSKPVRMLDKYKD $>$ P0A796

MIKKIGVLTSGGDAPGMNAAIRGVVRSALTEGLEVMGIYDGYLGLYEDRMVQLDRYSVSD MINRGGTFLGSARFPEFRDENIRAVAIENLKKRGIDALVVIGGDGSYMGAMRLTEMGFPC IGLPGTIDNDI KGTDYTIGFFTALSTVVEAIDRLRDTSSSHQRISVVEVMGRYCGDLTLA AAIAGGCEFVVVPEVEFSREDLVNEI KAGIAKGKKHAIVAITEHMCDVDELAHFIEKETG RETRATVLGHIQRGGSPVPYDRILASRMGAYAIDLLLAGYGGRCVGIQNEQLVHHDI IDA IENMKRPFKGDWLDCAKKLY

$>Q 05097$

AWKGEVLANNEAGQVTS I I YNPGDVITIVAAGWASYGPTQKWGPQGDREHPDQGLICHDA FCGALVMKIGNSGTI PVNTGLFRWVAPNNVQGAITLIYNDVPGTYGNNSGSFSVNIGKDQ $\mathrm{S}$

$>$ P0A6K6

MKRAF IMVLDSFGIGATEDAERFGDVGADTLGHIAEACAKGEADNGRKGPLNLPNLTRLG LAKAHEGSTGF I PAGMDGNAEVIGAYAWAHEMSSGKDTPSGHWEIAGVPVLFEWGYFSDH ENSFPQELLDKLVERANLPGYLGNCHSSGTVILDQLGEEHMKTGKP I FYTSADSVFQIAC HEETFGLDKLYELCEIAREELTNGGYNIGRVIARPF I GDKAGNFQRTGNRHDLAVEPPAP TVLQKLVDEKHGQVVSVGKIADIYANCGITKKVKATGLDALFDATI KEMKEAGDNTIVFT NFVDFDSSWGHRRDVAGYAAGLELFDRRLPELMSLLRDDDILILTADHGCDPTWTGTDHT REHI PVLVYGPKVKPGSLGHRETFADIGQTLAKYFGTSDMEYGKAMF

$>$ P18330

MTDYKATLNLPDTAFPMKAGLPQREPQILQRWNS IGLYGKLREIGKDRPKFVLHDGPPYA NGTIHIGHALNKI LKDMILRSKTLSGFDAPYVPGWDCHGLPIEHKVEVTYGKNLGADKTR ELCRAYATEQIEGQKSEFIRLGVLGEWDNPYKTMNFKNEAGEIRALAEIVKGGFVFKGLK PVNWCFDCGSALAEAEVEYEDKKSSTIDVAFPIADDAKLAEAFGLASLAKPAAIVIWTTT PWTI PANQALNVHPEFTYALVDVGDRLLVLAEEMVESCLARYELQGSVIATATGSALELI NFRHPFYDRLSPVYLADYVELGSGTGIVHCSPAYGVDDFVICKKYGMVNDDI INPVQSNG VYVPSLEFFGGQF I FKADQP I IEKLREVGALMQTAAIQHSYMHCWRHKTPLIYRATAQWF IGMDKEPTSGDTLRVRSLKAIEDTKFVPSWGQARLHSMIANRPDWCISRQRNWGVPI PFF LNKESGELHPRTVELMEVVAQRVEQQGIEAWFKLDAAELLGDEAPLYDKISDTLDVWFDS GTTHWHVLRGSHPMGHETGPRADLYLEGSDQHRGWFHSSLLTGCAIDNHAPYRELLTHGF TVDETGRKMSKSLKNVIEPKKINDTLGADIMRLWVASTDYSGEIAVSDQILARSADAYRR IRNTARFMLSNLTGFNPASDLLPAEDMLALDRWAVDRTLLLQRELQEHYGEYRFWNVYSK I HNFCVQELGGFYLDI I KDRQYTTGANSKARRSAQTALYH I SEALVRWIAPILAFTADEL WEYLPGERNESVMLNTWYEGLTELPADFELGREYWEGVMAVKVAVNKELEVQRAAKAVGG NLQAEVTLFAEDGLTADLAKLSNELRFVLITSTASLAPFTQAPADAVATEVPGLKLKVVK SAFPKCARCWHCREDVGVNPEHPEICGRCVDNISGEGEVRHYA

$>$ Q92 I38

MKFTLSWLKQFLETSASVTEIAEALTAIGLEVEEVIDKAAELQKFEVAYITNIKPHPSAD KLKLCDVETKSGMLQIVCGARNARAGIKVVLANIGIEI PNGKFKI KESVIRGEKSCGMLC SEEELLLASESEGI IELSEDAVVGENFTKYYGLDDP I FVINVTPNRGDALGVYGIARDLA 
AKGIGILKELEISVIKSTFISKMKLNVQDKEACPLFTFREIRNLKNKPSPDWLRKLLKNV GVKTISSLVDVTNYISYSFGQPMHAYDADRIKGGITVARHCEKHSDEAISGQQNEIATAA LQPRNDVAKCHALNGKEYLLTENDLVI KDESGIQGLAGVIGEADSSCTDSTTNI ILEAAC FNAKMVAASGRRFQIDTDARYRNERNIDRNFTEKALDIATNLILS I CGNGEVSEVVKVGE KESQKKPLDFSACYLEKITGIKLNIKAIEAILNKLGFITDVKGEI IKVIAPSWRHDITIL EDIAEEIARIYGYDKIESIKLPELDQDNNKLREHKRISSFKRILASKGYDEVVTNSFMSS EDAKLFAELKEELFLLNP IS I GDNYMRPT ILPNLLS IVSKNLARS I KDMAFFEVGPSF ID LNTEATYLTAI ISGSYNNKNPHSFGRGYDVFDLKGDLELVADYAGLS IDKCIATNGTALP QYYHPTRAVNIGLGKNLLGHFGQIHPKILKYYDINQEIFAFELNITNLPLIKAKFGKRDE FAVSDFQANFRDYAF IVGQDHRVGEI ISY INNFNKKLIKSVILFDIYSGDKLPEGKKS IA VKIELQADDRTLSDTDLNSFSKDLVAAISQKFQGTLRE

$>$ P31101

MFCFQCQETAKNTGCTVKGMCGKPEETANLQDLLIFVLRGIAIYGEKLKELGQPDRSNDD FVLQGLFATITNANWDDARFEAMISEGLARRDKLRNAFLAVYKAKNGKDFSEPLPEAATW TGDSTAFAEKAKSVGILATENEDVRSLRELLI IGLKGVAAYAEHAAVLGFRKTEIDEFML EALASTTKDLSVDEMVALVMKAGGMAVTTMALLDEANTTTYGNPEITQVNIGVGKNPGIL ISGHDLKDMAELLKQTEGTGVDVYTHGEMLPANYYPAFKKYPHFVGNYGGSWWQQNPEFE SFNGPILLTTNCLVPLKKENTYLDRLYTTGVVGYEGAKHIADRPAGGAKDFSALIAQAKK CPPPVEIETGS IVGGFAHHQVLALADKVVEAVKSGAIKRFVVMAGCDGRQKSRSYYTEVA ENLPKDTVILTAGCAKYRYNKLNLGDIGGI PRVLDAGQCNDSYSLAVIALKLKEVFGLDD INDLPVSYDIAWYEQKAVAVLLALLFLGVKGIRLGPTLPAFLSPNVAKVLVENFNIKPIG TVQDDIAAMM

$>Q 51434$

MQLIQELSQARDRGLYQEVGKLTRELHNAIVDFQIDPHSPHAQEMSQIADATDRLSYVVE MTEKAANRTMDLVEQSAPLVNQLGDDSRELHQEWQRFMRREIDADGFRELAKRIEQFLVR SGENAGQLSSQLNDILLAQDYQDLTGQVIKRVTKLVTEVESNLVKLVWMAGQVDRYAGIE HDHVSMRHQAALERSAKGEGPQVAAEKREDVVSGQDDVDDLLSSLGF

$>$ POA2W5

TATFDKVADI IAETSEIDRATITPESHTIDDLGIDSLDFLDIVFAIDKEFGIKIPLEKWT QEVNEGKVSTEEYFVLKNLCAKIDELKAAKA

$>$ P09373

SELNEKLATAWEGFTKGDWQNEVNVRDFIQKNYTPYEGDESFLAGATEATTTLWDKVMEG VKLENRTHAPVDFDTAVASTITSHDAGYINKQLEKIVGLQTEAPLKRAL I PFGGIKMIEG SCKAYNRELDPMIKKIFTEYRKTHNQGVFDVYTPDILRCRKSGVLTGLPDAYGRGRI IGD YRRVALYGIDYLMKDKLAQFTSLQADLENGVNLEQTIRLREEIAEQHRALGQMKEMAAKY GYDISGPATNAQEAIQWTYFGYLAAVKSQNGAAMSFGRTSTFLDVYIERDLKAGKITEQE AQEMVDHLVMKLRMVRFLRTPEYDELFSGDP IWATES IGGMGLDGRTLVTKNSFRFLNTL YTMGPSPEPNMTILWSEKLPLNFKKFAAKVS IDTSSLQYENDDLMRPDFNNDDYAIACCV SPMIVGKQMQF FGARANLAKTMLYAINGGVDEKLKMQVGPKSEP IKGDVLNYDEVMERMD HFMDWLAKQYITALNI I HYMHDKYSYEASLMALHDRDVIRTMACGIAGLSVAADSLSAIK YAKVKPIRDEDGLAIDFEIEGEYPQFGNNDPRVDDLAVDLVERFMKKIQKLHTYRDAIPT QSVLTITSNVVYGKKTGNTPDGRRAGAPFGPGANPMHGRDQKGAVASLTSVAKLPFAYAK DGISYTFS IVPNALGKDDEVRKTNLAGLMDGYFHHEAS IEGGQHLNVNVMNREMLLDAME NPEKYPQLTIRVSGYAVRFNSLTKEQQQDVITRTFTQSM

$>$ P09378

MAFCNNANLLNVFVRHIANNQLRSLAEVATVAHQLKLLKDDFFASDQQAVAVADRYPQDV FAEHTHDFCELVIVWRGNGLHVLNDRPYRITRGDLFYIHADDKHSYASVNDLVLQNI IYC PERLKLNLDWQGA I PGFNASAGQPHWRLGSMGMAQARQVIGQLEHESSQHVPFANEMAEL LFGQLVMLLNRHRYTSDSLPPTSSETLLDKLITRLAASLKSPFALDKFCDEASCSERVLR QQFRQQTGMTINQYLRQVRVCHAQYLLQHSRLLISDISTECGFEDSNYFSVVFTRETGMT PSQWRHLNSQKD

$>$ Q9ZKG9

MQKSLITTPIYYVNDI PHIGHAYTTLIADTLKKYYTLQGEEVFFLTGTDEHGQKIEQSAR LRNQSPKAYADS ISAIFKNQWDFFNLDYDGFIRTTDSEHQKCVQNAFEIMFEKGDIYKGT YSGYYCVSCESYCAVSKVDNTDSKVLCPDCLRETTLLEEESYFFKLSAYEKPLLEFYAKN PEAILPIYRKNEVTSFIEQGLLDLS ITRTSFEWGI PLPKKMNDPKHVVYVWLDALLNYAS ALGYLNGLDNKMAHFERARHIVGKDILRFHAIYWPAFLMSLNLPLFKQLCVHGWWTIEGV KMSKSLGNVLDAQKLAMEYGIEELRYFLLREVPFGQDGDFSKKALVERINANLNNDLGNL LNRLLGMAKKYFNYSLKSTKITAYYPKELEKAHQILDNANSFVPKMQLHKALEELFNIYD 
FLNKLIAKEEPWVLHKNNESEKLEALLSLIANTLLQSSFLLYAFMPKSAMKLASAFRVEI TPNNYERFFKAKKLQDMVLQDTEPLFSKIEKIEKIEKIEKIEKIEKGEEALAEKAEKKEK EKAPPTQENYISIEDFKKVEIKVGLIKEAQR IEKSNKLLRLKVDLGENRLRQI ISGIALD YEPESLVGQMVCVVANLKPAKLMGEMSEGMILAVRDNDNLALISPTREKIAGSLIS

$>$ P24251

TLPSGHPKSRLIKKFTALGPYIREGKCEDNRFFFDCLAVCVNVKPAPEVREFWGWWMELE AQESRFTYSYQFGLFDKAGDWKSVPVKDTEVVERLEHTLREFHEKLRELLTTLNLKLEPA DDFRDEPVKLTA

$>Q 00594$

MLGQMMRNQLVIGSLVEHAARYHGAREVVSVETSGEVTRSCWKEVELRARKLASALGKMG LTPSDRCATIAWNNIRHLEVYYAVSGAGMVCHTINPRLF IEQITYVINHAEDKVVLLDDT FLPI IAE IHGSLPKVKAFVLMAHNNSNASAQMPGL IAYEDLIGQGDDNYIWPDVDENEAS SLCYTSGTTGNPKGVLYSHRSTVLHSMTTAMPDTLNLSARDTILPVVPMFHVNAWGTPYS AAMVGAKLVLPGPALDGASLSKLIASEGVSIALGVPVVWQGLLAAQAGNGSKSQSLTRVV VGGSACPASMIREFNDIYGVEVIHAWGMTELSPFGTANTPLAHHVDLSPDEKLSLRKSQG RPPYGVELKIVNDEGIRLPEDGRSKGNLMARGHWVIKDYFHSDPGSTLSDGWFSTGDVAT IDSDGFMT I CDRAKDI I KSGGEW ISTVELES IAIAHPH IVDAAVIAARHEKWDERPLLIA VKSPNSELTSGEVCNYFADKVARWQI PDAAIFVEELPRNGTGKILKNRLREKYGDILLRS SSSVCE

$>$ POA944

MNTISSLETTDLPAAYHIEQRAHAFPWSEKTFASNQGERYLNFQLTQNGKMAAFAITQVV LDEATLFNIAVDPDYQRQGLGRALLEHLIDELEKRGVATLWLEVRASNAAAIALYESLGF NEATIRRNYYPTTDGREDAI IMALPISM

$>$ P0A8F4

MTDQSHQCVI IGIAGASASGKSLIASTLYRELREQVGDEHIGVI PEDCYYKDQSHLSMEE RVKTNYDHPSAMDHSLLLEHLQALKRGSA IDLPVYSYVEHTRMKETVTVEPKKVI I LEGI LLLTDARLRDELNFS IFVDTPLDICLMRR I KRDVNERGRSMDSVMAQYQKTVRPMFLQFI EPSKQYADI IVPRGGKNRIAIDILKAKISQFFE

$>$ P18775

MKTKI PDAVLAAEVSRRGLVKTTAIGGLAMASSALTLPFSRIAHAVDSAI PTKSDEKVIW SACTVNCGSRCPLRMHVVDGEIKYVETDNTGDDNYDGLHQVRACLRGRSMRRRVYNPDRL KYPMKRVGARGEGKFERISWEEAYDI IATNMQRL I KEYGNES IYLNYGTGTLGGTMTRSW PPGNTLVARLMNCCGGYLNHYGDYSSAQIAEGLNYTYGGWADGNSPSDIENSKLVVLFGN NPGETRMSGGGVTYYLEQARQKSNARMI I IDPRYTDTGAGREDEWI P IRPGTDAALVNGL AYVMITENLVDQAFLDKYCVGYDEKTLPASAPKNGHYKAYILGEGPDGVAKTPEWASQIT GVPADKI I KLAREIGSTKPAFISQGWGPQRHANGEIATRAISMLAILTGNVGINGGNSGA REGSYSLPFVRMPTLENPIQTSISMFMWTDAIERGPEMTALRDGVRGKDKLDVPIKMIWN YAGNCLINQHSEINRTHEILQDDKKCELIVVIDCHMTSSAKYADILLPDCTASEQMDFAL DASCGNMSYVI FNDQVIKPRFECKTIYEMTSELAKRLGVEQQFTEGRTQEEWMRHLYAQS REAIPELPTFEEFRKQGIFKKRDPQGHHVAYKAFREDPQANPLTTPSGKIEIYSQALADI AATWELPEGDVIDPLPIYTPGFESYQDPLNKQYPLQLTGFHYKSRVHSTYGNVDVLKAAC RQEMWINPLDAQKRGIHNGDKVRI FNDRGEVHIEAKVTPRMMPGVVALGEGAWYDPDAKR VDKGGCINVLTTQRPSPLAKGNPSHTNLVQVEKV

$>$ P0A8U6

AEWSGEYISPYAEHGKKSEQVKKITVS I PLKVLKILTDERTRRQVNNLRHATNSELLCEA FLHAFTGQPLPDDADLRKERSDEIPEAAKEIMREMGINPE

$>$ P0A6LO MTDLKASSLRALKLMDLTTLNDDDTDEKVIALCHQAKTPVGNTAA I CIYPRF I P IARKTL KEQGTPEIRIATVTNFPHGNDDIDIALAETRAAIAYGADEVDVVFPYRALMAGNEQVGFD LVKACKEACAAANVLLKVI IETGELKDEALIRKASEIS IKAGADF I KTSTGKVAVNATPE SARIMMEVIRDMGVEKTVGFKPAGGVRTAEDAQKYLAIADELFGADWADARHYRFGASSL LASLLKALGHGDGKSASSY

$>$ POA 8 F8

SKPFKLNSAFKPSGDQPEAIRRLEEGLEDGLAHQTLLGVTGSGKTFTIANVIADLQRPTM VLAPNKTLAAQLYGEMKEFF PENAVEYFVSYYDYYQPEAYVPSSDTF IEKDASVNEHIEQ MRLSATKAMLERRDVVVVASVSA IYGLGDPDLYLKMMLHLTVGMI IDQRAILRRLAELQY ARNDQAFQRGTFRVRGEVIDIFPAESDDIALRVELFDEEVERLSLFDPLTGQIVSTIPRF TIYPKTHYVTPRERIVQAMEEIKEELAARRKVLLENNKLLEEQRLTQRTQFDLEMMNELG YCSGIENYSRFLSGRGPGEPPPTLFDYLPADGLLVVDESHVTI PQIGGMYRGDRARKETL 
VEYGFRLPSALDNRPLKFEEFEALAPQTIYVSATPGNYELEKSGGDVVDQVVRPTGLLDP I IEVRPVATQVDDLLSEIRQRAAINERVLVTTLTKRMAEDLTEYLEEHGERVRYLHSDID TVERMEI IRDLRLGEFDVLVGINLLREGLDMPEVSLVAILDADKEGFLRSERSLIQTIGR AARNVNGKAILYGDKITPSMAKAIGETERRREKQQKYNEEHGITPQGLNKKVVDILALGQ NIAKTKAKGRGKSRPIVEPDNVPMDMSPKALQQKIHELEGLMMQHAQNLEFEEAAQIRDQ LHQLRELFIAAS

$>$ P43341

MATLFIADLHLCVEEPAITAGFLRFLAGEARKADALYILGDLFEAWIGDDDPNPLHRKMA AAIKAVSDSGVPCYFIHGNRDFLLGKRFARESGMTLLPEEKVLELYGRRVLIMHGDTLCT DDAGYQAFRAKVHKPWLQTLFLALPLFVRKRIAARMRANSKEANSSKSLAIMDVNQNAVV SAMEKHQVQWLIHGHTHRPAVHELIANQQPAFRVVLGAWHTEGSMVKVTADDVELIHFPF $>$ P0A6L4

ATNLRGVMAALLTPFDQQQALDKASLRRLVQFNIQQGIDGLYVGGSTGEAFVQSLSEREQ VLEIVAEEAKGKIKLIAHVGCVSTAESQQLAASAKRYGFDAVSAVTPFYYPFSFEEHCDH YRAI IDSADGLPMVVYNI PALSGVKLTLDQINTLVTLPGVGALKQTSGDLYQMEQIRREH PDLVLYNGYDEIFASGLLAGADGGIGSTYNIMGWRYQGIVKALKEGDIQTAQKLQTECNK VIDLLIKTGVFRGLKTVLHYMDVVSVPLCRKPFGPVDEKYLPELKALAQQLMQERG $>$ P06110 MTGMSNVSKLAGEPSGQEFLVFTLGNEEYGIDILKVQEIRGYDQVTRIANTPAFIKGVTN LRGVIVPIVDLRVKFCEGDVEYDDNTVVIVLNLGQRVVGIVVDGVSDVLSLTAEQIRPAP EFAVTLSTEYLTGLGALGERMLILVNIEKLLNSEEMALLDIAASHVA

$>$ P13402

MADKTI FNDHLNTNPKTNLRLWVAFQMMKGAGWAGGVFFGTLLLIGFFRVVGRMLPIQEN QAPAPNITGALETGIELIKHLV

$>$ Q9EXP1

MAQLPDPQKLLRNFSRCSNWEEKYLYI IELGAGLAPLSDAQRQDGNRVSGCQSQVWIDLA SNEQGNVVLHGDSDAAIVKGLIAIVFSLYQGLSVREIVELDVRPFFASLALTQHLTPSRS QGLEAMLRAVRARASALI

$>$ P43927

MI IVTSGHVDHGKTALLKALTGTSTAHLPEEKKRGMTIDLGYAYLPLENKVLGFIDVPGH EKFLSNMLAGLGGVHYAMLIVAADEGVAVQTKEHLAILRQLQFHEI IVVITKADRTNSAQ IESLIQTIKQDYSFLRNANYFVTSAETGQGISELRHYLANLAELADTQKPFRYAIDRVFS VKGAGTVVTGTAFSGTVKVNDEIYLSTGQKIRIKAIHAQNTSSEQGIAGQRLALNLNADL DRTPMKRGDWLLQNEPLPPTDRISVQILAEVPLNESQPVHIYHGASRTTGKLTLLQGKNA AKNDRTLAEI ILDSPLFLAFGDKLILRSGDTKTLIAGARVLEINSPKRHKRTEVRLNFLA NLALAENASQR IALTLQHNATTARQLMWTEQLTSLQLDKALAERDAVRYQDWCFNPNYVQ EKTQQILTALNIYHEQHNDQLGVSKARLYRMATLNQPENLIHHF IDEMLDDGRLQQTRGW IHLPEHKIQFNTEEKSRWTDVLNEFEKANGQAIWVRDMANALAIDES IMRNFMYKAGKLG YLTPIVKDRFFLTETLYAYARLIKQIAEEKGKVSVNEVRDKLNFGRKLTVQLMEYFDRMG FLRRKGNDHILRDKNVFDL

$>$ P13266

MRTKI IAI I PARSGSKGLRNKNALMLIDKPLLAYTIEAALQSEMFEKVIVTTDSEQYGAI AESYGADFLLRPEELATDKASSFEF IKHALS IYTDYESFALLQPTSPFRDSTHI IEAVKL YQTLEKYQCVVSVTRSNKPSQI IRPLDDYSTLSFFDLDYSKYNRNS IVEYHPNGAIFIAN KQHYLHTKHFFGRYSLAYIMDKESSLDIDDRMDFELAITIQQKKNRQKIDLYQNIHNRIN EKRNEFDSVSDITLIGHSLFDYWDVKKINDIEVNNLGIAGINSKEYYEYI IEKELIVNFG EFVFIFFGTNDIVVSDWKKEDTLWYLKKTCQYIKKKNAASKIYLLSVPPVFGRIDRDNRI INDLNSYLRENVDFAKFISLDHVLKDSYGNLNKMYTYDGLHFNSNGYTVLENEIAEIVK $>$ P06987

MSQKYLFIDRDGTLISEPPSDFQVDRFDKLAFEPGVI PELLKLQKAGYKLVMITNQDGLG TQSFPQADFDGPHNLMMQI FTSQGVQFDEVLI CPHLPADECDCRKPKVKLVERYLAEQAM DRANSYVIGDRATDIQLAENMGITGLRYDRETLNWPMIGEQLTRRDRYAHVVRNTKETQI DVQVWLDREGGSKINTGVGFFDHMLDQIATHGGFRMEINVKGDLYIDDHHTVEDTGLALG EALKIALGDKRGICRFGFVLPMDECLARCALDISGRPHLEYKAEFTYQRVGDLSTEMIEH FFRSLSYTMGVTLHLKTKGKNDHHRVESLFKAFGRTLRQAIRVEGDTLPSSKGVL

$>$ P43859

MSEKYVVTWDMFQMHARRLSERLLPASQWKGI IAVSRGGLFPAAVLARELGLRHIETVCI ASYHDHNNQGELQVLHAAQVPNGGEGFIVVDDLVDTGNTARAIRQMYPNAKFVTVFAKPA GAELVDDYVIDI PQNTWIEQPWDLGLTFVPPLSRK 
$>$ P34895

MSSAPAAGTASTSRFFKSHVSETDPDIFSAIQKEFGRQQHEIELIASENIVSQAVLDAAG SVLTNKYAEGYPGKRYYGGCQYVDIVEDIAIDRAKKLFNCEFANVQPNSGSQANQGVFNA LAQPGDTILGLSLAAGGHLTHGAPVNQSGKWFKAVHYMVKPDSHLIDMDEVRKLAQEHKP RIIIAGGSAYPRKIDFAAFRAIADEVGAIFLVDMAHFAGLVAAGLIPSPFPHAHVVTTTT HKTLRGPRGGMILTNDADIAKKINSAIFPGIQGGPLMHVIAGKAVAFGEALRPDFKVYIK QVMDNARALGEVLVQNGFALVSGGTDTHLVLVDLRPKKLTGTKAEKALGRANITCNKNGI PFDPEKPMVTSGIRLGSPAGTTRGFGVAEFQEIGRLISEVLDGVAKNGEDGNGAVEAAVK AKAIALCDRFPIYA

$>$ P0A955

MKNWKTSAES ILTTGPVVPVIVVKKLEHAVPMAKALVAGGVRVLEVTLRTECAVDAIRAI AKEVPEAIVGAGTVLNPQQLAEVTEAGAQFAISPGLTEPLLKAATEGTIPLI PGISTVSE LMLGMDYGLKEFKFFPAEANGGVKALQAIAGPFSQVRFCPTGGISPANYRDYLALKSVLC IGGSWLVPADALEAGDYDRITKLAREAVEGAKL

$>$ P0A9D8

MQQLQNI IETAFERRAEITPANADTVTREAVNQVIALLDSGALRVAEKIDGQWVTHQWLK KAVLLSFRINDNQVIEGAESRYFDKVPMKFADYDEARFQKEGFRVVPPAAVRQGAFIARN TVLMPSYVNIGAYVDEGTMVDTWATVGSCAQIGKNVHLSGGVGIGGVLEPLQANPTI IED NCFIGARSEVVEGVIVEEGSVISMGVYIGQSTRIYDRETGEIHYGRVPAGSVVVSGNLPS KDGKYSLYCAVIVKKVDAKTRGKVGINELLRTID

$>$ P23536

MPFALHGI PVSRGVAIGRAHLLAPAALDVSHYLVDEDQLDAEVERLRAARAAVRAELAAL KRDLPRDAPEELGAFLDVHAMILDDEALAREPEALIRGRRYNAEWALTTRLEELMHQFDE IEDEYLRERKTDIRQVVERILKALAGAPVLVPAPVPALAADGEAATGVIVVAHDIAPADM LQFRHTVFHGFVTDMGGRTSHTAIVARSLDI PAAVGVQSASELIRQDDWI I IDGDAGLVI VDPTAI ILEEYRHRQSERALEKKRLQRLRHTPAVTLDGLEIDLLANIEMAEDAGAALAAG AVGVGLFRSEFLFMNRRDELPGEDEQFQAYRGAVDAMHGLPVTIRTIDIGADKPLDARGD EFETALNPALGLRAIRWSLSEPGMFLTQLRALLRASAFGPVRLLVPMLAHASEIDQTLAL IAKAKRQLDERGEAYDPGMKVGAMIEIPAAVLLLPLFLRKMDFLSIGTNDLIQYTLAIDR ADNAVAHLFDPLHPAVLQLVARTIREANRAGVPVAVCGEMAGDPSMTRRLLGMGLREFSM HPAQLLRVKQEILHAHCERLEPLVDQVLQAFDPEEQARPLRSWHDPEAIRVF

$>Q 83 \mathrm{KQ} 3$

MNIQALLSEKVRQAMIAAGAPADCEPQIRQSAKVQFGDYQANGMMAVAKKLGMAPRQLAE QVLTHLDLNGIASKVEIAGPGF INI FLDPAFLAEHVQQALASDRLGVATPEKQTIVVDYS APNVAKEMHVGHLRSTI IGDTAVRTLEFLGHKVIRANHVGDWGTQFGMLIAWLEKQQQEN AGEMELADLEGFYRDAKKHYDEDEEFAERARNYVVKLQSGDEYFREMWRKLVDITMTQNQ ITYDRLNVTLTRDDVMGESLYNPMLPGIVADLKAKGLAVESEGATVVFLDVFKNKEGEPM GVI IQKKDGGYLYTTTDIACAKYRYETLHADRVLYYIDSRQHQHLMQAWAIVRKAGYVPE SVPLEHHMFGMMLGKDGKPFKTRAGGTVKLADLLDEALERARRLVAEKNPDMPADELEKL ANAVGIGAVKYADLSKNRTTDYIFDWDNMLAFEGNTAPYMQYAYTRVLSVFRKAEINEEQ LAAAPVI IREDREAQLAARLLQFEETLTVVAREGTPHVMCAYLYDLAGLFSGFYEHCPIL SAENEEVRNSRLKLAQLTAKTLKLGLDTLGIETVERM

$>$ P0A 8 G9

PKKNEAPASFEKALSELEQIVTRLESGDLPLEEALNEFERGVQLARQGQAKLQQAEQRVQ ILLSDNEDASLTPFTPDNE

$>$ P05827

MDLRDLKTFLHLAESRHFGRSARAMHVSPSTLSRQIQRLEEDLGQPLFVRDNRTVTLTEA GEELRVFAQQTLLQYQQLRHTIDQQGPSLSGELHIFCSVTAAYSHLPPILDRFRAEHPSV EIKLTTGDAADAMEKVVTGEADLAIAGKPETLPGAVAFSMLENLAVVLIAPALPCPVRNQ VSVEKPDWSTVPF IMADQGPVRRRIELWFRRNKISNPMIYATVGGHEAMVSMVALGCGVA LLPEVVLENSPEPVRNRVMILERSDEKTPFELGVCAQKKRLHEPLIEAFWKILPNHK $>085300$

MGLPLTFDDNNQCLLLLDSDIFTS IEAKDD IWLLNGMI I PLSPVCGDS IWRQIMVINGEL AANNEGTLAYIDAAETLLLIHAITDLTNTYHI ISQLESFVNQQEALKNILQEYAKV $>\mathrm{P} 0 \mathrm{C} 058$

MRNFDLSPLMRQWIGFDKLANALQNAGESQSFPPYNIEKSDDNHYRITLALAGFRQEDLE IQLEGTRLSVKGTPEQPKEEKKWLHQGLMNQPFSLSFTLAENMEVSGATFVNGLLHIDLI RNEPEPIAAQRIAISERPALNS

$>$ Q65V95 
MRI IMATYTTSDFKPGLKFMQDGEPCVIVENEFVKPGKGQAFTRTRIRKLISGKVLDVNF KSGTSVEAADVMDLNLNYSYKDEDFWYFMHPETFEQYSADSKAVGDAEKWLLDQAECI IT LWNGSPISVTPPNFVELEVVDTDPGLKGDTAGTGGKPATLSTGAVVKVPLFIQIGEVIKV DTRSGEYVSRVK

$>$ P60919

MAEKPKKPQKLRARLPRGLADRGPAEIAATRAMVEKIREVYERYGFEPVETPAFEYTDAL GKFLPDQDRPNEGVFSLQDDDEQWISLRYDLTAPLARYVAENFDQLPKPHRSYRFGWVFR NEKPGPGRFRQFMQFDADTVGAPTAAADAEMCMMAADTMEALGI PRGSYVVKVNNRKVLD GVLESIGLGGDENAGRRLTVLRAIDKSDKFAPEEIKKLLGPGRWDGGEEGKGDFTKGAML SDDQSELILRATSPSFIAGRFNADGSGGISNIDTVELLRSTADNETLKQGCDELAVIADL LDACGYGATSANPDVRVVIDPSVVRGLEYYTGPVYEVELLLDTKDEKGRPVRFGSVGGGG RYDGLVSRFRGEPVPATGFS IGVSRLQAALTLIGQLGNKPQAGPVVVTVFGGEIAGYQKM VATLRKAGIRAELYLGNPKHSLGQQMKYADKRNSPCA I IQGSDEKQQGIVQI KDL I LGAE LASLEKDRDEYLKKQAEAQF SCNEDEMVAKVQELLQRRGVAWG

$>Q 01551$

TKYNEAYCDVLIVGAGPAGVMAAAHLLSYGTTARPHRVRIFDATKEVNGSDESTESLSTD VIADALNSGASGPEKDAASTTEDLPMLVTTLQVSDVLHDTGDDTKIAYRETATEQQVLLL ADTTANTSSTMNPRSMCEAGCRFHQIYQGHCFPEYELDSERLRSVDGRAQVLEDEHETGQ LRLERLGRPEELLELDEENSMSVVTNLKAAPYKFLMKDVDENFPGELSTSGGKTTS ISAD ESAIDAALHAVWDADDLGAAWHLDEASGLRAVDWNAAQWFKSGQPWTPDAAKSLQEGRVF LAGDARHRHPPLTGIGKNTS IADCYNLTWKLLGVLLGVARADPARTYVAERVYIRMRAAT DIAVDAEMESLAAKWITVQLTLSRSWISSAKEAERWDAVLRDSAMSASKPMWTTSDMRAS FDAGLMGHGHAHDHVTPTIKEFASSS ISRS I SELASTSWWESRGWGNGGPFESLMEDARW TGAVESNCRYAAYDRDAPVLHEHVAWVTRFTSRARTAVLEAAVGQAHVVDCWDVGLVEPA LDDLDSAGAGLHVAHHADQWPAQLDEAVWPRESLSDWRIVTDTSATGEGYQTSPREAPGD YADLNADNAKAHFNGQFAGHKAYGDAAAADGGGCHGR I LVGPAVRGRHLHREI PLGEECQ RAAQPLFKEV

(2) 20 extracell proteins

$>005465$

MKLFTSTLTAKKSSTHKPLISLALSVLISTLLISETAQAADANDRLEQEVDKQAKQLMAQ YQI PGMAFGI IVDGKSHFYNYGLADKQRNQPVSEDTIFELGSVSKTFAATLASYSELNGT LSLDDTADKYI PYLKNSAIGNTKLISLVTYSAGGYHYRCLKTLENNKELLQYYKSWHPDF PVNSKRLYSNAS IGLFGYISALSMHSDYTKLIENTVLP SLKMTNTFVDVPANKMEDYAFG YNAAGEPIRVNPGMLDAEAYGIKSTSADMTRFMAANMGLVTVDSQMQQALDNNRKGYYRT KSFTQGLAWEMYPLPTTLQQLVEGNSTETILQPQP IQLNEPPTPVLNDVWVNKTGATNGF GAYIAYMPAKKTGMFILANKNYPNTERVKAAYTILDSVMN

$>Q 47473$

MKYLNCFISTGLAAFFLVNSTSVLAADCSSDLTSGISTKRIYYVAPNGSSSNNGNSFNSP MSFTAAMAAANPGELILLKPGTYTI PYTQGKGNTITFNKSGKEGSPIYVAAANCGRAVFD FSFPDSQWVQASYGFYVTGDYWYFKGIEVTRAGYQGAYVTGSHNTFENTAFHHNRNTGLE INNGGSYNTVINSDAYRNYDPKKNGSMADGFGPKQKQGQGNRFGGCRAWENSDDGFDLFD SPQKVVIENSWAFRNGINYWSDSSFAGNGNGFKLGGNQAVGNHRITRSVAFGNVSKGFDQ NNNAGGVTVINNTSYKNGINYGFGSNVKSGQKHYFRNNVSLSGSATVNNADAKSNSWDTG PVASASDFVSLDTSLATISRDNDGTLPETALFRLSTNSKLINAGTKESNISYSGSAPDLG AFERN

$>$ P15922

MKVITFSRRSALAS IVATCLMSTPALAATAQAPQKLQI PTLSYDDHSVMLVWDTPEDTSN ITDYQIYQNGQLIGLASQNNDKNSPAKPY ISAFYKSDAANFHHR IVLQNAKVDGLKAGTD YQFTVRTVYADGTTSNDSNTVTTTTTAVPKVINITQYGAKGDGTTLNTSAIQKAIDACPT GCRIDVPAGVFKTGALWLKSDMTLNLLQGATLLGSDNAADYPDAYKIYSYVSQVRPASLL NAIDKNSSAVGTFKNIRIVGKGI IDGNGWKRSADAKDELGNTLPQYVKSDNSKVSKDGIL AKNQVAAAVATGMDTKTAYSQRRSSLVTLRGVQNAYIADVTIRNPANHGIMFLESENVVE NSVIHQTFNANNGDGVEFGNSQNIMVFNSVFDTGDDS INFAAGMGQDAQKQEPSQNAWLF NNFFRHGHGAVVLGSHTGAGIVDVLAENNVITQNDVGLRAKSAPAIGGGAHGIVFRNSAM KNLAKQAVIVTLSYADNNGTIDYTPAKVPARFYDFTVKNVTVQDSTGSNPAIEITGDSSK DIWHSQF IFSNMKLSGVSPTS ISDLSDSQFNNLTFSNLRSGSSPWKFGTVKNVTVDGKTV $>$ P40600

MKKKLIYAAVVSALLAGCGGSDDNKGDTSSYLDYLLTGSNAVGPSALAARAWDGTLKFST ETADLSNPVSAMSTLDGWSTTQAIQIVPVTSSGITVQAPTTAEFGASVAPLYLLEVTFDS 
TALRPSGVKKVLTYGVDFVVAASAWQAEPGSAQAVEPLPCLANDSGHRTAERQSRRCLKA GSDYGNYKNNAGSNAQEQTINGLIALQEGLFKAATGIATDHVIFSDWFGTQSGADVLVAV KGAAASVLKADPVTLDAAKLWKQDAWEHQPARHLYPGRDRPTCLPDPAGCRAVPAAEQKD AIATAFGPVLRSTRLLKRPRSI PVPSSCLTSSPHRRPQVPGARPRPSPGTVPSQPVRHRQ CAEGVTRSDRRAGGGGRGSGPAGDADCRSDPPERAAGRGEQADWGDAHLRRQAAGRRAEH WSLQPAADAGRGAIRADACLRQGCPQHHHGCHHLSARRDLGQRERLRPGAGPDLEDLCRH AGGQEGGAGGDRSSAARRAWLRLSGSMDTVTTSDNPTPYLNLSYLTVARDNLKQSVAICW ACVWRLAWPTPRAIGTAGSLKVHFLGHSLGASRVPTCCGRQPDHRQRASGCPVQVRYRWP GHAGSHSAAAAELADFGPTI KMGVLTSGSAELKAGFTAYAPNCTDGGAYLLRQRVPAEPG RGHSATAATRCRVQLCGPVGAGFG

$>Q 60106$

MKIEKTALTVAIALAMSSLSAHAEDAWVSTHTQAAMSPPASTQVLAASSTSATTTGNAYT LNMTGSPRIDGAAVTALEADHPLHVEVALKLRNPDALQTFLAGVTTPGSALFGKFLTPSQ FTERFGPTQSQVDAVVAHLQQAGFTNIEVAPNRLLISADGTAGAATNGFRTS I KRFSANG REFFANDAPALVPASLGDSVNAVLGLQNVSVKHTLHHVYHPEDVTVPGPNVGTQAAAAVA AHHPQDFAAIYGGSSLPAATNTAVGI ITWGS ITQTVTDLNSFTSGAGLATVNSTITKVGS GTFANDPDSNGEWSLDSQDIVGIAGGVKQLIFYTSANGDSSSSGITDAGITASYNRAVTD NIAKLINVSLGEDETAAQQSGTQAADDA I FQQAVAQGQTFS IASGDAGVYQWSTDPTSGS PGYVANSAGTVKIDLTHYSVSEPASSPYVIQVGGTTLSTSGTTWSGETVWNEGLSAIAPS QGDNNQRLWATGGGVSLYEAAPSWQSSVSSSTKRVGPDLAFDAASSSGALIVVNGSTEQV GGTSLASPLFVGAFARIESAANNAIGFPASKFYQAFPTQTSLLHDVTSGNNGYQSHGYTA ATGFDEATGFGSFDIGKLNTYAQANWVTGGGGGSTNAPPVANFSVATTGLVATFTDSSTD SDGSIASHAWTFGDGSTSTATSPSHTYSAAGTYSVAETVTDNAGATSTKTSSVTVSSSGG TGGGTVLQNGVAATGLSAAKNGQLKYTVAI PSGAKSLKIAISGGTGDADLYVKFGSAPTT SSYDCRPYVTGNTESCSFASPQTGTYYVLLNGYAAFSGVSLKATWTN

$>Q 887 \mathrm{C6}$

MQALNSISSLQTSASLFPVSLNSDVSANTSTSSKELKAVIDQLVQALTQSGQLDETSPLG KMLAKAMAADGKSANS IDDITASLDKLIHEKLGDNFGASAGIGAGGGGGGIGGAGSGSGV GGGLSSDAGAGQSDLMSQVLNGLGKAVLDDLLTPSGEGGTTFSSDDMPTLEKVAQFMDDN KAQFPTRDGGSWMNELKEDNGLDAQETAQFRSALDVIGQQLGQQQGDASGVTSGGGLGSP VSDSSLGNPAIDANTGPAANGNASVDVGQLIGQLIDRGLQSVSSGGGLGTPVDNSTQPTG GTPAANPTGNVSNQDLGQLLSGLLQRGLEATLQDAGNTGADLQSSAAQVAAQLINALLQG TNNQTNQAVA

$>$ Q9RBS1

MSHSKIKAGGHGSSGIGNDFTPAKTPAPATPAPQSQQVNDLLGRGVGNALNKSNLGSDSQ TWTPGSTMVSLKSRSSSSHKPDTGGDTKPDSTSGGKRKRDDETDPNAETEGGKKKKKRDD ENDSSQAGGAGSSAGSSGSPEDALMNIALQRAIQRQTQTRQKMQEAMKI KDDDD $>Q 05205$

MNLSPSRTPICAALAAALLGAAALAPAHAAQRILQLSEDTTHSKPVSAASALRGTPLAKA GAADRVCEAGAKWLRVGFKQLKLAGYDSLVLTSSGGDKLVFEGQHWNQRSFTTRPLRGEC VDIQPYFSQPDSAFQLDRYDYSTVALDKATVVVAGAGDICDTSGNACQGTSDLIVS INPT AVFTAGDNAYNSGTLSEYNSRYAPTWGRFKALTSPSPGNHDYSTTGAKGYFDYFNGSGNQ TGPAGDRSKGYYSWDVGDWHFVSLNTMSGGTVAQAQIDWLKADLAANTKPCTAAYFHHPL LSRGSYSGYSQVKPFWDALYAAKADLVLVGHDHNYQRYGKMNPDKAAASDGIRQVLVGTG GRAFYGISGSHALLEASNDSTFGVLKLTLSATGYTGDFVPRAGSSYTDHFTGTCNKGSGN PPTQTLTLNSVRDVTVKSGGSRDNGATLYADGSDGGQVLRGLMAWNVSSAAGKTLTGAQV KLQVSDRSTGTYDLYRAGAAWTEANASYSGVSLGSKIGSVVPSATGAQS IALNAAGFSW $>$ P2 1347

MHPNYYLSPLAVAIALGIASPVKAADPI PLQKSSFSEVTQKFQLTLPGVMKGAVVSTNSL QFIRQHTDGNKVTHVRMQQQYAGFPVFGGYAILHSKNATPSLATAKSDEKMNGVIYDGLQ AELGQPKPSFVKNASMALQQFKDKYANKQVSEDQVTPMIYIDEKHQAHWAYKVSVLVIHD DRI PERPTAI IDAETNKPFVQWDDVKTEKVQAKGMGFGGNRKIGEYQFGKDLPLLEITRD SSVEMCFMENTDVKVVDMGHKYYSNNKPMQFTCKETPDTQSTKTYYTGYSADGYDRDNGA ASPTNDALYAGYVI KHMYHDWYGVEALTKSDGSPMQLVMRVHYGQGYENAYWDGKQMTFG DGDTMMYPLVSLGVGGHEVSHGFTEQHSGLEYFGQSGGMNESFSDMAAQAAEYYSVGKNS WQIGPEIMKEDSGYDALRYMDKPSRDGMS IDVADDYYGGLDVHYSSGVYNHLFYILANQP NWNLRMAFDVMVKANMDYWTPYSTFDEGGCGMLSAAKDLGYNLDDI KKSLSEVTINYQSC YVD

$>$ P13720 
MKKWFPAFLFLSLSGGNDALAGWHNVMFYAFNDYLTTNAGNVKVIDQPQLYI PWNTGSAT ATYYSCSGPEFASGVYFQEYLAWMVVPKHVYTNEGFNI FLDVQSKYGWSMENENDKDFYF FVNGYEWDTWTNNGARI CFYPGNMKQLNNKFNDLVFRVLLPVDLPKGHYNFPVRYIRGIQ HHYYDLWQDHYKMPYDQI KQLPATNTLMLSFDNVGGCQPSTQVLNIDHGS IVIDRANGNI ASQTLSIYCDVPVSVKISLLRNTPP I YNNNKF SVGLGNGWDS I ISLDGVEQSEEILRWYT AGSKTVKIESRLYGEEGKRKPGELSGSMTMVLSFP

$>$ Q93Q17

MQIQANTVGTQAVAHHSDATTGVGRMGQMEARQVATGQDAILLGSRSEPQKGQGLLSRLG AQLARPFVAI KEWISNLLGTDKRAAAPKAQTAVSPEDLQRLMKQAAFGSSLGGFAKADVL NNITGEQLGKDHASLATGNGPLRSLCTALQAVVIGSQQPQLRELATGLLARP IAGIPLQQ WGSVGGKVTELLTSAP PELLKEAMSQLHTAMGEVADLQRAVKAEVAGEPARSATTAAAVA PLQSGESEVNVEPADKALAEGLQEQFGLEAEQYLGEQPHGTYSDAEVMALGLYTNGEYQH LNRSLRQEKQLDAGQALIDQGMSTAFEKSTPTEQLIKTFRGTHGGDAFNEVAEGQVGHDV AYLSTSRDPKVATNFGGSGSISTIFGRSGIDVSDISVEGDEQEILYNKETDMRVLLSAKD ERGVTRRVLEEASLGEQSGHSKGLLDGLDLARGAGGADKPQEQDIRLKMRGLDLA

$>$ P08038

MMIFRFVTTLAASLPLLTFAAPISFSHAKNEAVKIYRDHPVSFYCGCEIRWQGKKGIPDL ESCGYQVRKNENRASRIEWEHVVPAWQFGHQLQCWQQGGRKNCTRTSPEFNQMEADLHNL TPAIGEVNGDRSNFSFSQWNGVDGVTYGQCEMQVNFKERTAMPPERARGAIARTYLYMSE QYGLRLSKAQSQLMQAWNNQYPVSEWECVRDQRIEKVQGNSNRFVREQCPN

$>$ Q99289

MMKKTITLLTALLPLASAVAEEPTLSPEMVSASEVISTQENQTYTYVRCWYRTSYSKDDP ATDWEWAKNEDGSYFTIDGYWWSSVSFKNMFYTNTSQNVIRQRCEATLDLANENADITFF AADNRFSYNHTIWSNDAAMQPDQINKVVALGDSLSDTGNIFNASQWRFPNPNSWFLGHFS NGFVWTEYIAKAKNLPLYNWAVGGAAGENQYIALTGVGEQVSSYLTYAKLAKNYKPANTL FTLEFGLNDFMNYNRGVPEVKADYAEALIRLTDAGAKNFMLMTLPDATKAPQFKYSTQEE IDKIRAKVLEMNEF I KAQAMYYKAQGYNITLFDTHALFETLTSAPEEHGFVNASDPCLDI NRSSSVDYMYTHALRSECAASGAEKFVFWDVTHPTTATHRYVAEKMLESSNNLAEYRF $>$ P74849

MVTSVRTQPPVIMPGMQTEIKTQATNLAANLSAVRESATATLSGEIKGQQLEDFPALIKQ ASLDALFKCGKDAEALKEVFTNSNNVAGKKAIMEFAGLFRSALNATSDSPEAKTLLMKVG AEYTAQI I KDGLKEKSAFGPWLPETKKAEAKLENLEKQLLDI I KNNTGGELSKLSTNLVM QEVMPYIASCIEHNFGCTLDPLTRSSLTQLVDKAAAKAVEALDMCHQKLTQEQGTSVGRE ARHLEMQTLI PLLLRNVFAQI PADKLPDPKI PEPAAGPVPDGGKKAEPTGINININIDSS NHSVDNSKHINNSRSHVDNSQRHIDNSNHDNSRKTIDNSRTFIDNSQRHGESHHSTNSSN VSHSHSRVDSTTHQTETAHSASTGTIDHGIAGKIDVTAHATAEAVTNSSSESKDGKVVTS EKGTTGETTSFDEVDGVTSKS I IGKPLQATVHGVDDNKQQSQTAEIVNVKPLASQLAGVE NVKIDTLQSDSTVITGNKAGTTDNDNSQTDKTGPFSGLKFKQNSFLSTVPSVTNMHS IHF NAREAFLGVIRKALEPDASTPFPVRRAFDGLRGEILPNDTIKSAALKAQCSDIDKHPELK AKMETLKEVITHHPQKEKLAEIALQFAREAGLTRQKGETDYVLSNVLDGLIGDGSWRAGP AYESYLNKPGVDRVITTVDGLHMQR

$>$ P2 3024

MQLLKQLFKKKFVKEEHDKKTGQEGMTLLEVI IVLGIMGVVSAGVVTLAQRAIDSQIMTK AAQSLNS IQVALTQTYRGLGNYPATADATAASKLTSGLVSLGKISSDEAKNPFNGTNMNI FSFPRNAAANKAFAISVDGLTQAQCKTLITSVGDMFPYIAIKAGGAVALADLGDFENSAA AAETGVGVIKSIAPASKNLDLTNITHVEKLCKGTAPFGVAFGNS

$>006949$

TKITLFPHNFRIQKQEATPLKEKSTEKNSLAKS I LAVKNHF IKLNSKLSERF ISHKNTES SATHFHRGSASEGRAVLTNKVVKNFMLQTLHDIDIRGSASKDPAYASQTREAILSAVYSK YKDQYCNLLISKGIDIAPFLKEIGEAAQNAGLPGATKNDVFSPSGAGANPF ITPLITSAY SKYPHMFTSQHQKASFNIYAEKI IMTEVVPLFNECAMPTPQQFQQILENIANKYIQNTP $>Q 11137$

MGSFLLKKAVGLSNISDLLDKSGIFYNYSTKVLPSFDYDTAGKHIAREDSTWNGKYVIGQ PAEVTYSFPKWEGKFNQFGNKNPYEFNELQKEHARKSLDAWSDIANI KFTEVAVGNVDGM KASDVKTDITFGNIYDPNGTFQAYATLPNTYAYGKDLSGQAWF SDYHYAGNTTPELGNYG RLTIIHEIGHTLGLMHPGDYNAGQNVPGYLKSDYAEDSRQYTVMSYWDEYETGAHFQGAY AGAPLLHDISAMQYLYGANTTTRTGDDVYGFNSNTGIDYYTATDSNDKLIFSVWDSGGND TFDFSGFYQDQLIDLRAGNF SDVGGLQKNVS IAQNVT I ENA IGGFGNDI I HGNDADNTLI GGEGDDI IYGHSGNNT I YGGRGQDTLHGGTGSNTF IYKEIADSLVTAADKIMDFKTGIDK 
IDLSTLIQDTFSSKILNFVDNFTGNAGEATLSYNEVTNASELAINAYGYNYNPDFKIDIV GFVNYETDFIV

$>Q 888 \mathrm{Y} 8$

MNPLRS IQHNIATPPISGGQPLDAVGPQAQQSHPKRISPSQLSQSAHQALERLSANAEHQ RLASLVRNALQDGTFQFQSSNHTQVTYKASICLPADTDTVRTDHLINNELTVQARLNDQS EYDIVSAHLHGSSKAISFDVPSPPPAHGSASSVLSERTHLGMSRVLSQDAVDSSSLETPL LSSPDHSRPPSQPKPVHIGSVRRDSGSLVSDNPVVQALLSFAQADQAFPPQAASIAGVQL EMRPRRDIEKALEEFKGAFTVVKAQLMSGANSSERVDEDVNADIHI PLLLKAIERGAAAF GPNAS IGQNSAKAFLASCAPKITSNDDVLSEF INQKLKGDDDLQVRLGAQELLHVATKKE FQLGGLAGS IGVSS ILGSAWELGASELLKNA I FGKNF SP SQYALQLAGIDSVPPLI IESM DTMCVLA I I KGMKGEEWSMSDLLPKALKAGAI SSVVSF PNNVLQYAGFKSRVGDLAANSV TTEAAI FGAASGI PPEVKESEELMRAGLFQSMKDGVMAHSGEGVDTKKTIERMTRHALDI APGESTAVKSMGLAS IVGMI PLIASNKATGLLSEQVLR I FRSAVFNP IEA IALNALALGG RVNVPGLFDSDNAKHARVVQTILARASQHMEAGDRDISAEELHQMLAPRSEFLRHVGSAI VNGMNASFEAI PALVRKLGYGEAPLAERI PYQDLAVPDTSRQPAP

$>$ P07863

MLKTISGTLALSLI IAASVHQAQAATTYNAVVSKSSSDGKTFKTIADAIASAPAGSTPFV ILIKNGVYNERLTITRNNLLLKGESRNGAVIAAATAAGTLKSDGSKWGTAGSSTITISAK DFSAQSLTIRNDFDFPANQAKSDSDSSKIKDTQAVALYVTKSGDRAYFKDVSLVGYQDTL YVSGGRSFFSDCRISGTVDF I FGDGTALFNNCDLVSRYRADVKSGNVSGYLTAPSTNINQ KYGLVITNSRVIRESDSVPAKSYGLGRPWHPTTTFSDGRYADPNAIGQTVFLNTSMDNHI YGWDKMSGKDKNGNTIWFNPEDSRFFEYKSYGAGAAVSKDRRQLTDAQAAEYTQSKVLGD WTPTLP

$>$ Q52473

VAFAGLTSKLTNLGNSAVGGVGGALQGVNTVASNATLQKNILLGTGDSLSVDAQAKASKE SDANGAKLIAMQAQETMKKQTMDVLNAIQAGKEDSTNKKISATATNAKGISY

(3) 4 fimbrium proteins

$>$ P19369

MPNFFRNGCIALVGSVAAMGAAHAEGGIAEAAGKALDSAQSDVTITAPKVMMVVATVVGV GILINMMRKA

$>$ P37920

MKHKLMTSTIASLMFVAGAAVAADPTPVSVSGGTIHFEGKLVYAACAVSTKSADQTVTLG QYRTASFTAIGDTTAQVPFS IVLNDCDPKVAATAAVAFSGQADNTNTNLLAVSSADNSTT ATGVGIEILDNTSS PLKPDGATFSAKQALVEGTNTLRFTARYKATAAATPGQANADATF I MKYE

$>$ P33783

MKKVTLFLFVVSLLPSTVLAWNTPGEDFSGELKLEGAVTSTRNPWVWKVGQGNESLEVKQ SRGVRDGEQGI PVALPALTVLLGKTTLTTPAGREGLSPGVSYGKGAEGFSLEWTAPGMAK VTLPVTGDKNVRAGTFTFRMQAAGVLRHMQDGQPVYTGVYDDLNANGLPGESTAMKTSDI PGTLQTMFSGEGPSWLQTMTVSGYSGVSHFSDASLRQVEGVYGAQIVAGGGELHLNGAMP ERWRVSLPVS

$>$ P11900

MKKTLIALAVAASAAVSGSVMAADWTEGQPGDI I IGGEITSPSVKWLWKTGEGLSSFSNT TNEIVKRKLNISVPTDELFLAAKMSDGI KGVFVGNTLI PKIEMASYDGSVITPSFTSNTA MDIAVKVKNSGDNTELGTLSVPLSFGAAVATI FDGDTTDSAVAHI IGGSAGTVFEGLVNP GRFTDQNIAYKWNGLSKAEMAGYVEKLMPGQSASTSYSGFHNWDDLSHSNYTSANKASYL SYGSGVSAGSTLVMNLNKDVAGRLEWVAPVTITVIYS

(4) 1 flagellum protein $>$ Q9R9R6

MAITSAGAGSGIDLESVISASVSAKKAQLQQPI ITKQNSTQITLSGIGQLKSS ISAFTDI LDKLSAPGAFNKRAINITQSKDDP I LKVEGKSGASNGQYNI IVNKLAETSRQEGIFDSST TPLATQDGQLTFKAGDKTFKVDVKAGDTLQDIRKS INSNGDNFGLSVNIVNTADGKAKLV IDSGISGDGKDLTITGDNAELGVFEAGGGVMSQTRAASSAEINVDGNVLKSDTNTFDDS I QDLKVTVLRVSDKDSAGDLKANKVDITTDKTSIQELVQQFVDGYNTLQDKMNSLGKRNTF VGGVKQDDGGALAGDSTTRAIESFMSNLLVSPSQNSGTYSTVFEIGI KMDNKGKLSLDKT KFGEAVDKNFDQVVALFGGEKGLASTLNSGLKEYTKSGGMLAQREDVLNSDLRALTQKTA TANAQLTKYEAALRAQYGSLDALLVKMNSSASALATLQTSYQKS

(5) 345 inner membrane proteins $>$ POADRO 
MSEALSLFSLFASSFLSATLLPGNSEVVLVAMLLSGISHPWVLVLTATMGNSLGGLTNVI LGRFFPLRKTSRWQEKATGWLKRYGAVTLLLSWMPVVGDLLCLLAGWMRISWGPVIF FLC LGKALRYVAVAAATVQGMMWWH

$>$ POADR2

MTSRFMLIFAAISGFIFVALGAFGAHVLSKTMGAVEMGWIQTGLEYQAFHTLAILGLAVA MQRRISIWFYWSSVFLALGTVLFSGSLYCLALSHLRLWAFVTPVGGVSFLAGWALMLVGA IRLKRKGVSHE

$>$ P21365

MS ITAQSVYRDTGNFFRNQFMTILLVSLLCAFITVVLGHVFSPSDAQLAQLNDGVPVSGS SGLFDLVQNMS PEQQQ I LLQASAASTFSGLIGNA I LAGGVILI IQLVSAGQRVSALRAIG ASAP ILPKLFILIFLTTLLVQIGIMLVVVPGI IMAILLALAPVMLVQDKMGVFASMRSSM RLTWANMRLVAPAVLSWLLAKTLLLLFASSFAALTPEIGAVLANTLSNLISAILLIYLFR LYMLIRQ

$>$ P77589

MSTRTPSSSSSRLMLTIGLCFLVALMEGLDLQAAGIAAGGIAQAFALDKMQMGWIFSAGI LGLLPGALVGGMLADRYGRKRILIGSVALFGLFSLATAIAWDFPSLVFARLMTGVGLGAA LPNLIALTSEAAGPRFRGTAVSLMYCGVPIGAALAATLGFAGANLAWQTVFWVGGVVPLI LVPLLMRWLPESAVFAGEKQSAPPLRALFAPETATATLLLWLCYFFTLLVVYMLINWLPL LLVEQGFQPSQAAGVMFALQMGAASGTLMLGALMDKLRPVTMSLLIYSGMLASLLALGTV SSFNGMLLAGFVAGLFATGGQSVLYALAPLFYSSQIRATGVGTAVAVGRLGAMSGPLLAG KMLALGTGTVGVMAASAPGILVAGLAVFILMSRRSRIQPC

$>$ POAE14

MTLFTTLLVLIFERLFKLGEHWQLDHRLEAFFRRVKHFSLGRTLGMTI IAMGVTFLLLRA LQGVLFNVPTLLVWLLIGLLCIGAGKVRLHYHAYLTAASRNDSHARATMAGELTMIHGVP AGCDEREYLRELQNALLWINFRFYLAPLFWLIVGGTWGPVTLMGYAFLRAWQYWLARYQT PHHRLQSGIDAVLHVLDWVPVRLAGVVYALIGHGEKALPAWFASLGDFHTSQYQVLTRLA QFSLAREPHVDKVETPKAAVSMAKKTSFVVVVVIALLTIYGALV

$>$ P64481

MSFIMTAEGHLLFSIACAVFAKNAELTPVLAQGDWWHIVPSAILTCLLPDIDHPKSFLGQ RLKWISKPIARAFGHRGFTHSLLAVFALLATFYLKVPEGWF I PADALQGMVLGYLSHILA DMLTPAGVPLLWPCRWRFRLPILVPQKGNQLERF I CMALFVWSVWMPHSLPENSAVRWSS QMINTLQIQFHRLIKHQVEY

$>085093$

MSALRLRKVDALLAQATRELGAGQSLGFSAAGQDAELTLLPLLADAGEPAGAVWLSTAIG PLLLSDAEALLSLLGDI PLTLGGEQQAWYWQLFNQRLSPTVARLLAPVEPLHNKPQAPTL GCRVQIRRGGEQLHAHMHATPDTLLRLLRSASWQARTRTVDESWSVASPLI IGEMSLTRE QIASLRPGDVVLPAHCQFDSAGQGFLSLAGRQWAAQTDQHAQRLFLRLSHEEHRHHEY $>$ P68699

MENLNMDLLYMAAAVMMGLAA I GAA I GIGILGGKFLEGAARQPDL I PLLRTQFF IVMGLV DAIPMIAVGLGLYVMFAVA

$>Q 09049$

MISESVVDLSRLQFAMTALYHFLFVPLTLGMTFLLAIMESVYVMTGKQVYKDMVKFWGKL FGINFALGVTTGITMEFQFGTNWAYYSHYVGDIFGAPLAIEGLTAFFLESTF IGMFFFGW DRLSKIQHLAVTWLVALGSNLSALWILVANGWMQHPVGAEFNFETMRMELVDFGALLLNP VAQVKFVHTVASGYVTGAVFVLAISSYYLLKKRDLGFARRSFAIASAFGMAS ILSVIVLG DESGYEVGEVQKAKLAAIEAEWETHPAPASFTLIGFPNEEEQRTDFAVKI PWVLGI IATR SLDEQVIGIKDLIADHEARIRNGMVRYGLLEELRAGNKSPEKIAAFNEVKDDLGYGLLLK KYTPNVVDASEEQIKQAAKDTI PSVASMFWSFRAMVGAGFAMLILFVCAFWASARKNEES KPWLLKFALYSLPLPWIATQTGWFVAEHGRQPWTIGGVLPTHLSASSLSTGDLWGSLIAL IAFYTLLLVVEMYLMIRFARLGP SSLHTGRYHFEQLEQHAVKHASP SQADPQQPVNA $>$ P07001 MRIGI PRERLTNETRVAATPKTVEQLLKLGFTVAVESGAGQLASFDDKAFVQAGAEIVEG NSVWQSEI ILKVNAPLDDEIALLNPGTTLVSFIWPAQNPELMQKLAERNVTVMAMDSVPR ISRAQSLDALSSMANIAGYRAIVEAAHEFGRFFTGQITAAGKVP PAKVMVIGAGVAGLAA IGAANSLGAIVRAFDTRPEVKEQVQSMGAEFLELDFKEEAGSGDGYAKVMSDAF I KAEME LFAAQAKEVDI IVTTALI PGKPAPKLITREMVDSMKAGSVIVDLAAQNGGNCEYTVPGEI FTTENGVKVIGYTDLPGRLPTQSSQLYGTNLVNLLKLLCKEKDGNITVDFDDVVIRGVTV IRAGEITWPAP P IQVSAQPQAAQKAAPEVKTEEKCTCSPWRKYALMALAI ILFGWMASVA PKEFLGHFTVFALACVVGYYVVWNVSHALHTPLMSVTNAISGI IVVGALLQIGQGGWVSF 
LSFIAVLIASINIFGGFTVTQRMLKMFRKN

$>$ P31064

MSWQQFKHAWLIKFWAPIPAVIAAGILSTYYFGITGTFWAVTGEFTRWGGQLLQLFGVHA EEWGYFKI I HLEGSPLTRIDGMMILGMFGGCFAAALWANNVKLRMPRSR IRIMQA I IGGI IAGFGARLAMGCNLAAFFTGI PQFSLHAWFFAIATAIGSWFGARFTLLPIFRIPVKMQKV SAASPLTQKPDQARRRFRLGMLVFFGMLGWALLTAMNQPKLGLAMLFGVGFGLLIERAQI CFTSAFRDMWITGRTHMAKA I I IGMAVSAIGIFSYVQLGVEPKIMWAGPNAVIGGLLFGF GIVLAGGCETGWMYRAVEGQVHYWWVGLGNVIGSTILAYYWDDFAPALATDWDKINLLKT FGPMGGLLVTYLLLFAALMLI IGWEKRFFRRAAPQTAKEI

$>$ POAFM6

GIFSRFADIVNANINALLEKAEDPQKLVRLMIQEMEDTLVEVRSTSARALAEKKQLTRRI EQASAREVEWQEKAELALLKEREDLARAALIEKQKLTDLI KSLEHEVTLVDDTLARMKKE IGELENKLSETRARQQALMLRHQAANSSRDVRRQLDSGKLDEAMARFESFERRIDQMEAE AESHSFGKQKSLDDQFAELKADDAISEQLAQLKAKMKQDNQ

$>$ P31069

MSHWATFKQTATNLWVTLRHDILALAVFLNGLLIFKTIYGMSVNLLDIFHIKAFSELDLS LLANAPLFMLGVFLVLNS I GLLFRAKLAWA IS I ILLLIALIYTLHFYPWLKFS IGFCIFT LVFLLILRKDFSHSSAAAGTIFAFISFTTLLFYSTYGALYLSEGFNPRIESLMTAFYFS I ETMSTVGYGDIVPVSESARLFTISVI ISGITVFATSMTSIFGPLIRGGFNKLVKGNNHTM HRKDHF IVCGHS I LA INT I LQLNQRGQNVTVISNL PEDDI KQLEQRLGDNADV I PGDSND SSVLKKAGIDRCRAILALSDNDADNAFVVLSAKDMSSDVKTVLAVSDSKNLNKIKMVHPD I ILSPQLFGSEILARVLNGEEINNDMLVSMLLNSGHGI FSDNDELETKADSKESAQK $>$ POACV4

MKYLLIFLLVLAIFVISVTLGAQNDQQVTFNYLLAQGEYRISTLLAVLFAAGFAIGWLIC GLFWLRVRVSLARAERKIKRLENQLSPATDVAVVPHSSAA

$>$ P4 6927

MTTIGTPLRTNATKVMMLGSGELGKEVVIELQPLGVEVIAVDRYDNAPAQQVAHRAYTIS MLDGNALRDLVEKEKPDF IVPEVEAIATATLVELEQEGYNVIPTAKATQLTMNREGIRRL AAEELGLKTSPYRFVDNFEQFQQAIQEIGI PCVVKPIMSSSGHGQSVIKSEADIQQAWDY SQQGGRAGGGRVIVEGFIKFDYEITQLTVRHIHGIVFSSHRHIQVDGDYRESWQPQQMSD IALKKAQETAEKITSALGGRGIFGVELFVCGDEI I FNEVSPRPHDTGIVTMASQELSQFA LHARAILGLPI PEIYRISPAASKAIVVEGKSDNVRFGGVDKVLAEIGTNIRLFGKGEVNG HRRLGVILARDENTVRALETSRRAYDKLDIQL

$>$ P07014

MRLEFSIYRYNPDVDDAPRMQDYTLEADEGRDMMLLDALIQLKEKDPSLSFRRSCREGVC GSDGLNMNGKNGLACITPISALNQPGKKIVIRPLPGLPVIRDLVVDMGQFYAQYEKIKPY LLNNGQNPPAREHLQMPEQREKLDGLYECILCACCSTSCPSFWWNPDKF IGPAGLLAAYR FLIDSRDTETDSRLDGLSDAFSVFRCHS IMNCVSVCPKGLNPTRAIGHIKSMLLQRNA $>$ POAGK1

MEWSLTQNKLLAFHRLMRTDKP IGALLLLWPTLWALWVATPGVPQLWILAVFVAGVWLMR AAGCVVNDYADRKFDGHVKRTANRPLPSGAVTEKEARALFVVLVLISFLLVLTLNTMTIL LS IAALALAWVYPFMKRYTHLPQVVLGAAFGWS I PMAFAAVSESVPLSCWLMFLANILWA VAYDTQYAMVDRDDDVKIGIKSTAILFGQYDKLI I GILQIGVLALMA I I GELNGLGWGYY WS ILVAGALFVYQQKLIANREREACFKAFMNNNYVGLVLFLGLAMSYWHF

$>$ POAFN6

MMHLYWVALKS IWAKEIHRFMRIWVQTLVPPVITMTLYFI I FGNLIGSR I GDMHGFSYMQ FIVPGLIMMSVITNAYANVASSFFGAKFQRNIEELLVAPVPTHVI IAGYVGGGVARGLFV GILVTAISLFFVPFQVHSWVFVALTLVLTAVLFSLAGLLNGVFAKTFDDISLVPTFVLTP LTYLGGVFYSLTLLPPFWQGLSHLNPIVYMISGFRYGFLGINDVPLVTTFGVLVVFIVAF YLICWSLIQRGRGLRS

$>Q 43961$

MNGSKKIYLGIGLVALLMI F IYWLMPKDTANASSQIESTNASA I IATSPGQQNQLSENTT PFGSVSQHDTQVNCQLQLNAANHLIVNEQTRNCFEYFLTQYGEKSLTQIDQDIKNYFTQS LPQPARDQAQDLWQRYLKYREELGNLKEPAIAKTDIAYYRAVFTSRQMLRQRFFSATEIA GLFGSEDIYNQYTLERMA I LNNSKLSEIEKAKQLKALFDQLPQDWKANLEQLSKLDDLKQ LTTS IKKNGGSAQELHDMRTNLVGHDATARLEQLDVERSNWKSNVTQYLDERQTILNSNM SDTAKQNAISALRSKNFTAPQDQIRVQAFESAKDQGQSLPFSE $>$ P03819

MDSHTLIQALIYLGSAALIVPIAVRLGLGSVLGYLIAGCI IGPWGLRLVTDAES ILHFAE 
IGVVLMLF I IGLELDPQRLWKLRAAVFGCGALQMVI CGGLLGLFCMLLGLRWQVAELIGM TLALSSTA IAMQAMNERNLMVTQMGRSAFAVLLFQDIAAI PLVAMI PLLATSSASTTMGA FALSALKVAGALVLVVLLGRYVTRPALRFVARSGLREVFSAVALFLVFGFGLLLEEVGLS MAMGAFLAGVLLASSEYRHALESDIEPFKGLLLGLFF IGVGMS IDFGTLLENPLRIVILL LGFLI IKIAMLWLIARPLQVPNKQRRWFAVLLGQGSEFAFVVFGAAQMANVLEPEWAKSL TLAVALSMAATP ILLVILNRLEQSSTEEAREADEIDEEQPRVI IAGFGRFGQITGRLLLS SGVKMVVLDHDPDHIETLRKFGMKVFYGDATRMDLLESAGAAKAEVLINAIDDPQTNLQL TEMVKEHFPHLQI IARARDVDHYIRLRQAGVEKPERETFEGALKTGRLALESLGLGPYEA RERADVFRRFNIQMVEEMAMVENDTKARAAVYKRTSAMLSEI ITEDREHLSLIQRHGWQG TEEGKHTGNMADEPETKPSS

$>$ P0AD65

MKLQNSFRDYTAESALFVRRALVAFLGILLLTGVLIANLYNLQIVRFTDYQTRSNENRIK LVPIAPSRGI IYDRNGI PLALNRTIYQIEMMPEKVDNVQQTLDALRSVVDLTDDDIAAFR KERARSHRFTS I PVKTNLTEVQVARFAVNQYRFPGVEVKGYKRRYYPYGSALTHVIGYVS KINDKDVERLNNDGKLANYAATHDIGKLGIERYYEDVLHGQTGYEEVEVNNRGRVIRQLK EVPPQAGHDIYLTLDLKLQQYIETLLAGSRAAVVVTDPRTGGVLALVSTPSYDPNLFVDG ISSKDYSALLNDPNTPLVNRATQGVYPPASTVKPYVAVSALSAGVITRNTTLFDPGWWQL PGSEKRYRDWKKWGHGRLNVTRSLEESADTFFYQVAYDMGIDRLSEWMGKFGYGHYTGID LAEERSGNMPTREWKQKRFKKPWYQGDTI PVGIGQGYWTATPIQMSKALMILINDGIVKV PHLLMSTAEDGKQVPWVQPHEPPVGDIHSGYWELAKDGMYGVANRPNGTAHKYFASAPYK IAAKSGTAQVFGLKANETYNAHKIAERLRDHKLMTAFAPYNNPQVAVAMILENGGAGPAV GTLMRQILDHIMLGDNNTDLPAENPAVAAAEDH

$>$ P11696

TDIRTGLTDEECQEIHEMNMLGMHAYWS I GL IANALAYAWRPFHQGRAGNRLEDHAPDYV RSALT

$>$ POAC98

MGNTKLANPAPLGLMGFGMTTILLNLHNVGYFALDG I I LAMGIFYGGIAQIFAGLLEYKK GNTFGLTAFTSYGSFWLTLVAILLMPKLGLTDAPNAQFLGVYLGLWGVFTLFMFFGTLKG ARVLQFVFFSLTVLFALLA I GNIAGNAA I IHFAGWIGLICGASA IYLAMGEVLNEQFGRT VLPIGESH

$>$ POA6AO

MTPGEVRRLYFI IRTFLSYGLDELI PKMRITLPLRLWRYSLFWMPNRHKDKLLGERLRLA LQELGPVWI KFGQMLSTRRDLF P PHIADQLALLQDKVAPFDGKLAKQQIEAAMGGLPVEA WFDDFEIKPLASAS IAQVHTARLKSNGKEVVIKVIRPDI LPVIKADLKLIYRLARWVPRL LPDGRRLRPTEVVREYEKTLIDELNLLRESANAIQLRRNFEDSPMLYI PEVYPDYCSEGM MVMERIYGIPVSDVAALEKNGTNMKLLAERGVQVFFTQVFRDSFFHADMHPGNIFVSYEH PENPKYIGIDCGIVGSLNKEDKRYLAENF IAFFNRDYRKVAELHVDSGWVPPDTNVEEFE FAIRTVCEPIFEKPLAEISFGHVLLNLFNTARRFNMEVQPQLVLLQKTLLYVEGVGRQLY PQLDLWKTAKPFLESWI KDQVGI PALVRAFKEKAP FWVEKMPELPELVYDSLRQGKYLQH SVDKIARELQSNHVRQGQSRYFLGIGATLVLSGTFLLVSRPEWGLMPGWLMAGGLIAWFV GWRKTR

$>$ P77682

MLKLFAKYTS IGVLNTLIHWVVFGVCIYVAHTNQALANFAGFVVAVSFSFFANAKFTFKA STTTMRYMLYVGFMGTLSATVGWAADRCALPPMITLVTFSAISLVCGFVYSKFIVFRDAK $>$ P33695

MPNETLHIDIGVCTYRRPELAETLRSLAAMNVPERARLRVIVADNDAEPSARALVEGLRP EMPFDILYVHCPHSNISIARNCCLDNSTGDFLAFLDDDETVSGDWLTRLLETARTTGAAA VLGPVRAHYGPTAPRWMRSGDFHSTLPVWAKGEIRTGYTCNALLRRDAASLLGRRFKLSL GKSGGEDTDFFTGMHCAGGTIAFSPEAWVHEPVPENRASLAWLAKRRFRSGQTHGRLLAE KAHGLRQAWNIALAGAKSGFCATAAVLCFPSAARRNRFALRAVLHAGVISGLLGLKEIEQ YGAREVTSA

$>$ P 77757

MFEIHPVKKVSVVI PVYNEQESLPELIRRTTTACESLGKEYEILLIDDGSSDNSAHMLVE ASQAENSHIVS ILLNRNYGQHSA IMAGFSHVTGDL I ITLDADLQNPPEEI PRLVAKADEG YDVVGTVRQNRQDSWFRKTASKMINRLIQRTTGKAMGDYGCMLRAYRRHIVDAMLHCHER STFIPILANIFARRAIEI PVHHAEREFGESKYSFMRLINLMYDLVTCLTTTPLRMLSLLG SI IAIGGF S IAVLLVILRLTFGPQWAAEGVFMLFAVLFTF IGAQF IGMGLLGEYIGRIYT DVRARPRYFVQQVIRPSSKENE

$>$ P 69739 
MNNEETFYQAMRRQGVTRRSFLKYCSLAATSLGLGAGMAPKIAWALENKPRI PVVWIHGL ECTCCTESFIRSAHPLAKDVILSLISLDYDDTLMAAAGTQAEEVFEDI ITQYNGKY ILAV EGNPPLGEQGMFCISSGRPFIEKLKRAAAGASAI IAWGTCASWGCVQAARPNPTQATPID KVITDKPI I KVPGCPP I PDVMSAI ITYMVTFDRLPDVDRMGRPLMFYGQRIHDKCYRRAH FDAGEFVQSWDDDAARKGYCLYKMGCKGPTTYNACSSTRWNDGVSFPIQSGHGCLGCAEN GFWDRGSFYSRVVDI PQMGTHSTADTVGLTALGVVAAAVGVHAVASAVDQRRRHNQQPTE TEHQPGNEDKQA

$>$ POACXO

MGLVIKAALGALVVLLIGVLAKTKNYYIAGLI PLFPTFALIAHYIVASERGIEALRATI I FSMWS I I PYFVYLVSLWYFTGMMRLPAAFVGSVACWGISAWVLI I CW I KLH

$>$ POAER 8

MFHLDTLATLVAATLTLLLGRKLVHSVSFLKKYTI PEPVAGGLLVALALLVLKKSMGWEV NFDMSLRDPLMLAFFATIGLNANIASLRAGGRVVGI FLIVVVGLLVMQNAIGIGMASLLG LDPLMGLLAGS ITLSGGHGTGAAWSKLFIERYGFTNATEVAMACATFGLVLGGLIGGPVA RYLVKHSTTPNGI PDDQEVPTAFEKPDVGRMITSLVLIETIALIAICLTVGKIVAQLLAG TAFELPTFVCVLFVGVILSNGLS IMGFYRVFERAVSVLGNVSLSLFLAMALMGLKLWELA SLALPMLA I LVVQTIFMALYAI FVTWRMMGKNYDAAVLAAGHCGFGLGATPTAIANMQA I TERFGPSHMAFLVVPMVGAFFIDIVNALVIKLYLMLPIFA

$>$ P43531

MSRTTTVDGAPASDTDKQS ISQPNQFIKRGTPQFMRVTLALFSAGLATFALLYCVQPILP VLSQEFGLTPANSS ISLS ISTAMLA IGLLFTGPLSDAIGRKPVMVTALLLAS ICTLLSTM MTSWHGILIMRALIGLSLSGVAAVGMTYLSEE IHPSFVAFSMGLYISGNS IGGMSGRLIS GVFTDFFNWRIALAAIGCFALASALMFWKILPESRHFRPTSLRPKTLFINFRLHWRDRGL PLLFAEGFLLMGSFVTLFNYIGYRLMLSPWHVSQAVVGLLSLAYLTGTWSSPKAGTMTTR YGRGPVMLFSTGVMLFGLLMTLFSSLWLIFAGMLLFSAGFFAAHSVASSWIGPRAKRAKG QASSLYLFSYYLGSS IAGTLGGVFWHNYGWNGVGAF IALMLVIALLVGTRLHRRLHA

$>$ P33915

MSRLSPVNQARWARFRHNRRGYWSLWIFLVLFGLSLCSELIANDKPLLVRYDGSWYFPLL KNYSESDFGGPLASQADYQDPWLKQRLENNGWVLWAP IRFGATS INFATNKPFPSPPSRQ NWLGTDANGGDVLARILYGTRI SVLFGLMLTLCSSVMGVLAGALQGYYGGKVDLWGQRFI EVWSGMPTLFLI ILLSSVVQPNFWWLLAITVLFGWMSLVGVVRAEFLRTRNFDYIRAAQA LGVSDRS I ILRHML PNAMVATLTFLPF ILCSS ITTLTSLDFLGFGLPLGS PSLGELLLQG KNNLQAPWLGITAFLSVAILLSLLIFIGEAVRDAFDPNKAV

$>$ P42590

MSDTKRNTIGKFGLLSLTFAAVYSFNNVINNNIELGLASAPMFFLATIFYFIPFCLIIAE FVSLNKNSEAGVYAWVKSSLGGRWAF ITAYTYWFVNLFFFTSLLPRVIAYASYAFLGYEY IMTPVATTI ISMVLFAFSTWVSTNGAKMLGP ITSVTSTLMLLLTLSYILLAGTALVGGVQ PADA ITVDAMI PNFNWAFLGVTTWI FMAAGGAESVAVYVNDVKGGSKSFVKVI I LAGIFI GVLYSVSSVLINVFVSSKELKFTGGSVQVFHGMAAYFGLPEALMNRFVGLVSFTAMFGSL LMWTATPVKI FFSEI PEGI FGKKTVELNENGVPARAAWIQFLIVI PLMI I PMLGSNTVQD LMNT I INMTAAASMLP PLF IMLAYLNLRAKLDHLPRDFRMGSRRTGI IVVSMLIA I FAVG FVASTFPTGANILTI IFYNVGGIVIFLGFAWWKYSKYI KGLTAEERHIEATPASNVD $>$ P18392

MKKLFIQFYLLLFVCFLVMSLLVGLVYKFTAERAGKQSLDDLMNSSLYLMRSELREI PPH DWGKTLKEMDLNLSFDLRVEPLSKYHLDDISMHRLRGGEIVALDDQYTFLQRI PRSHYVL AVGPVPYLYYLHQMRLLDIALIAF IA ISLAFPVF IWMRPHWQDMLKLEAAAQRFGDGHLN ERIHFDEGSSFERLGVAFNQMADNINALIASKKQLIDGIAHELRTPLVRLRYRLEMSDNL SAAESQALNRDISQLEALIEELLTYARLDRPQNELHLSEPDLPLWLSTHLADIQAVTPDK TVRI KTLVQGHYAALDMRLMERVLDNLLNNALRYCHSTVETSLLLSGNRATLIVEDDGPG IAPENREHI FEPFVRLDPSRDRSTGGCGLGLAIVHS IALAMGGTVNCDTSELGGARFSFS WPLWHNI PQFTSA

$>$ POAGMO

METPQPDKTGMHILLKLASLVVILAGIHAAADI IVQLLLALFFAIVLNPLVTWFIRRGVQ RPVAITIVVVVMLIALTALVGVLAASFNEF I SMLPKFNKELTRKLFKLQEMLPFLNLHMS PERMLQRMDSEKVVTFTTALMTGLSGAMASVLLLVMTVVFMLFEVRHVPYKMRFALNNPQ IHIAGLHRALKGVSHYLALKTLLSLWTGVIVWLGLELMGVQFALMWAVLAFLLNYVPNIG AVISAVPPMIQVLLFNGVYECILVGALFLVVHMVIGNILEPRMMGHRLGMSTMVVFLSLL IWGWLLGPVGMLLSVPLTSVCKIWMETTKGGSKLAILLGPGRPKSRLPG $>$ POAFPO 
MLVYWLDIVGTAVFAISGVLLAGKLRMDPFGVLVLGVVTAVGGGTIRDMALDHGPVFWVK DPTDLVVAMVTSMLTIVLVRQPRRLPKWMLPVLDAVGLAVFVGIGVNKAFNAEAGPLIAV CMGVITGVGGGI IRDVLAREI PMILRTEIYATACI IGGIVHATAYYTFSVPLETASMMGM VVTLLIRLAAIRWHLKLPTFALDENGR

$>$ P 64590

MDWYLKVLKNYVGFRGRARRKEYWMFILVNI I FTFVLGLLDKMLGWQRAGGEGILTTIYG ILVFLPWWAVQFRRLHDTDRSAWWALLFL I PF IGWLI I IVFNCQAGTPGENRFGPDPKLE $\mathrm{P}$

$>$ Q9ZHG0

MTLLSFGFSPVFFSVMAFCI ISRSKFYPQRTRNKVIVLILLTFFICFLYPLTKVYLVGSY GIFDKFYLFCFISTLIAIAINVVILTINGAKNERN

$>$ P56579

MIETITHGAEWF IGLFQKGGEVFTGMVTGILPLLISLLVIMNALINF IGQHRIERFAQRC AGNPVSRYLLLPCIGTFVFCNPMTLSLGRFMPEKYKPSYYAAASYSCHSMNGLFPH INPG ELFVYLGIASGLTTLNLPLGPLAVSYLLVGLVTNFFRGWVTDLTTAIFEKKMGIQLEQKV HLAGATS

$>$ P33927

MMPEIGNGLLCLALGIALLLSVYPLWGVARGDARMMASSRLFAWLLFMSVAGAFLVLVNA FVVNDFTVTYVASNSNTQLPVWYRVAATWGAHEGSLLLWVLLMSGWTFAVAIFSQR I PLD IVARVLAIMGMVSVGFLLFILFTSNPFSRTLPNFP IEGRDLNPLLQDPGLIFHPPLLYMG YVGFSVAFAFAIASLLSGRLDSTYARFTRPWTLAAWIFLTLGIVLGSAWAYYELGWGGWW FWDPVENASFMPWLVGTALMHSLAVTEQRASFKAWTLLLAISAFSLCLLGTFLVRSGVLV SVHAFASDPARGMF ILAFMVLVIGGSLLLFAARGHKVRSRVNNALWSRESLLLANNVLLV AAMLVVLLGTLLPLVHKQLGLGS IS I GEPFFNTMFTWLMVPFALLLGVGPLVRWGRDRPR KIRNLLI IAF I STLVLSLLLPWLFESKVVAMTVLGLAMACWIAVLAIAEAALRISRGTKT TFSYWGMVAAHLGLAVTIVGIAFSQNYSVERDVRMKSGDSVDIHEYRFTFRDVKEVTGPN WRGGVATIGVTRDGKPETVLYAEKRYYNTAGSMMTEAAIDGGITRDLYAALGEELENGAW AVRLYYKPFVRWIWAGGLMMALGGLLCLFDPRYRKRVSPQKTAPEAV

$>$ P69826

MENKSARAKVQAFGGFLTAMVI PNIGAFIAWGF ITALF I PTGWLPNEHFAKIVGPMITYL LPVMIGSTGGHLVGGKRGAVMGGIGTIGVIVGAEI PMFLGSMIMGPLGGLVIKYVDKALE KRIPAGFEMVINNFSLGIAGMLLCLLGFEVIGPAVLIANTFVKECIEALVHAGYLPLLSV INEPAKVLFLNNAIDQGVYYPLGMQQASVNGKS I FFMVASNPGPGLGLLLAFTLFGKGMS KRSAPGAMI I HFLGGIHELYFPYVLMKPLTI IAMIAGGMSGTWMFNLLDGGLVAGPSPGS IFAYLALTPKGSFLATIAGVTVGTLVSFAITSLILKMEKTVETESEDEFAQSANAVKAMK QEGAFSLSRVKRIAFVCDAGMGSSAMGATTFRKRLEKAGLAIEVKHYAIENVPADADIVV THASLEGRVKRVTDKPLILINNYIGDPKLDTLFNQLTAEHKH

$>$ P 67143

MNEI ISAAVLLILIMDPLGNLPIFMSVLKHTEPKRRRAIMVRELLIALLVMLVFLFAGEK ILAFLSLRAETVS ISGGI ILFLIAIKMIFPSASGNSSGLPAGEEPF IVPLAI PLVAGPT I LATLMLLSHQYPNQMGHLVIALLLAWGGTFVILLQSSLFLRLLGEKGVNALERLMGLILV MMATQMFLDGIRMWMKG

$>$ POAGOO

MMTQPMPGKPAEDAENELDIRGLFRTLWAGKLWI I GMGLAFALIALAYTFFARQEWSSTA ITDRPTVNMLGGYYSQQQFLRNLDVRSNMASADQP SVMDEAYKEFVMQLASWDTRREFWL QTDYYKQRMVGNSKADAALLDEMINNIQF I PGDFTRAVNDSVKLIAETAPDANNLLRQYV AFASQRAASHLNDELKGAWAART IQMKAQVKRQEEVAKAIYDRRMNS IEQALKIAEQHNI SRSATDVPAEELPDSEMFLLGRPMLQARLENLQAVGPAFDLDYDQNRAMLNTLNVGPTLD PRFQTYRYLRTPEEPVKRDSPRRAFLMIMWGIVGGLIGAGVALTRRCSK

$>$ POAF32

MIQYLNVFFYDIYPYICATVFFLGSWLRYDYGQYTWRASSSQMLDKRGMVIWSNLFHIGI LGIFFGHLFGMLTPHWMYAWFLPVAAKQLMAMVLGGI CGVLTLIGGAGLLWRRLTNQRVR ATSTTPDI I IMS ILLIQCLLGLSTIPFSAQYPDGSEMMKLVGWAQS IVTFRGGSSEMLNG VAFVFRLHLVLGMTIFLLFPFTRLVHVWSAPFEYFTRRYQIVRSRR

$>$ P56580

MTHIRIEKGTGGWGGPLELKATPGKKIVYITAGTRPAIVDKLAQLTGWQAIDGFKEGEPA EAEIGVAVIDCGGTLRCGIYPKRRIPTINIHSTGKSGPLAQYIVEDIYVSGVKEENITVV GDATPQP SSVGRDYDTSKKITEQSDGLLAKVGMGMGSTVAVLFQSGRDTIDTVLKTILPF MAFVSALIGI IMASGLGDW IAHGLAPLASHPLGLVMLALI CSFPLLSPFLGPGAVIAQVI 
GVLIGVQIGLGNI PPHLALPALFAINAQAACDF I PVGLSLAEARQDTVRVGVPSVLVSRF LTGAPTVLIAWFVSGFIYQ

$>$ POAET5

MLYIDKATILKFDLEMLKKHRRAIQF IAVLLFIVGLLCISFPFVSGDILSTVVGALLICS GIALIVGLFSNRSHNFWPVLSGFLVAVAYLLIGYFFIRAPELGIFAIAAFIAGLFCVAGV IRLMSWYRQRSMKGSWLQLVIGVLDIVIAWIFLGATPMVSVTLVSTLVGIELIFSAASLF SFASLFVKQQ

$>$ P76111

MNQSLTLAFLIAAGIGLVVQNTLMVRITQTSSTILIAMLLNSLVGIVLFVSILWFKQGMA GFGELVSSVRWWTLI PGLLGSFFVFAS ISGYQNVGAATTIAVLVASQLIGGLMLDIFRSH GVPLRALFGPICGAILLVVGAWLVARRSF

$>$ P33790

MNEVYVIAGGEWLRNNLNAIAAFMGTWTWDSIEKIALTLSVLAVAVMWVQRHNVMDLLGW VAVFVLISLLVNVRTSVQI IDNSDLVKVHRVDNVPVGLAMPLSLTTRIGHAMVASYEMIF TQPDSVTYSKTGMLFGANLIVKSTDFLSRNPEI INLFQDYVQNCVLGDIYLNHKYTLEDL MASADPYTLIFSRPSPLRGVYDNNNNFITCKDASVTLKDRLNLDTKTGGKTWHYYVQQIF GGRPDPDLLFRQLVSDSYSYFYGSSQSASQIMRQNVTMNALKEGITSNAARNGDTASLVS LATTSSMEKQRLAHVS IGHVTMRNLPMVQTILTGIAIGIFPLLILAAVFNKLTLSVLKGY VFALMWLQTWPLLYAILNSAMTFYAKQNGAPVVLSELSQIQLKYSNLASTAGYLSAMIPP LSWMMVKGLGAGFSSVYSHFASSS ISPTASAAGSVVDGNYSYGNMQTENVNGFSWSTNST TSFGQMMYQTGSGATATQTRDGNMVMDASGAMSRLPVGINATRQIAAAQQEMAREASNRA ESALHGFSSSIASAWNTLSQFGSNRGSSDSVTGGADSTMSAQDSMMASRMRSAVESYAKA HNISNEQATRELASRSTNASLGLYGDAYAKGHLGI SVLGNGGGVGLQAGAKAS IDGSDLD SHEASSGSRASHDARHDIDARATQDFKEASDYFTSRKVSESGSHTDNNADSRVDQLSAAL NSAKQSYDQYTTNMTRSHEYAEMASRTESMSGQMSEDLSQQFAQYVMKNAPQDVEAILTN TSSPEIAERRRAMAWSFVQEQVQPGVDNTWRESRRDI GKGMESVPSGGGSQDI IADHQGH QA I IEQRTQDSNIRNDVKHQVDNMVTEYRGNIGDTQNS I RGEENIVKGQYSELQNHHKTE ALTQNNKYNEEKLAQERI PGADSPKELLEKAKSYQHKE

$>$ P32129

MANLLNKF IMTR ILAA ITLLLS IVLTILVTIFCSVP I I IAGIVKLLLPVPVIWRKVSRFC DFMMYCWCEGLAVLLHLNPHLQWEVHGLEGLSKKNWYLLICNHRSWADIVVLCVLFRKHI PMNKYFLKQQLAWVPFLGLACWSLDMPFMKRYSRAYLLRHPERRGKDVETTRRSCEKFRL HPTTIVNFVEGSRFTQEKHQQTHSTFQNLLPPKAAGIAMALNVLGKQFDKLLNVTLCYPD NNRQP FFDMLSGKLTRIVVHVDLQP IADELHGDY INDKS FKRHFQQWLNSLWQEKDRLLT SLMSSQRQNK

$>$ P 67153

MVQKPLIKQGYSLAEEIANSVSHGIGLVFGIVGLVLLLVQAVDLNASATAITSYSLYGGS MILLFLASTLYHAI PHQRAKMWLKKFDHCAIYLLIAGTYTPFLLVGLDSPLARGLMIVIW SLALLGILFKLTIAHRFKILSLVTYLAMGWLSLVVIYEMAVKLAAGSVTLLAVGGVVYSL GVIFYVCKRI PYNHAIWHGFVLGGSVCHFLAIYLYIGQA

$>$ P77858

MRQTLCDGYLVIFALAQAVILLMLTPLFTGISRQIRARMHSRRGPGIWQDYRDIHKLFKR QEVAPTSSGLMFRLMPWVLISSMLVLAMALPLFITVSPFAGGGDLITLIYLLALFRFFFA LSGLDTGSPFAGVGASRELTLGILVEPMLILSLLVLALIAGSTHIEMISNTLAMGWNSPL TTVLALLACGFACF IEMGKI PFDVAEAEQELQEGPLTEYSGAGLALAKWGLGLKQVVMAS LFVALFLPFGRAQELSLACLLTSLVVTLLKVLLI FVLAS IAENTLARGRFLL IHHVTWLG FSLAALAWVFWLTGL

$>$ POAG14

MELLSEYGLFLAKIVTVVLAIAA IAA I IVNVAQRNKRQRGELRVNNLSEQYKEMKEELAA ALMDSHQQKQWHKAQKKKHKQEAKAAKAKAKLGEVATDSKPRVWVLDFKGSMDAHEVNSL REEITAVLAAFKPQDQVVLRLESPGGMVHGYGLAASQLQRLRDKNIPLTVTVDKVAASGG YMMACVADKIVSAPFAIVGS I GVVAQMPNFNRFLKSKDIDIELHTAGQYKRTLTLLGENT EEGREKFREELNETHQLFKDFVKRMRPSLDIEQVATGEHWYGQQAVEKGLVDEINTSDEV ILSLMEGREVVNVRYMQRKRLIDRFTGSAAESADRLLLRWWQRGQKPLM

$>$ P37147

MRWLPFIAIFLYVYIEISIFIQVAHVLGVLLTLVLVIFTSVIGMSLVRNQGFKNFVLMQQ KMAAGENPAAEMI KSVSLI IAGLLLLLPGFFTDFLGLLLLLPPVQKHLTVKLMPHLRFSR MPGGGFSAGTGGGNTFDGEYQRKDDERDRLDHKDDRQD

$>$ P0A7B1 
GQEKLYIEKELSWLSFNERVLQEAADKSNPLIERMRFLGIYSNNLDEFYKVRFAELKRRI I ISEEQGSNSHSRHLLGKIQSRVLKADQEFDGLYNELLLEMARNQI FLINERQLSVNQQN WLRHYFKQYLRQHITPILINPDTDLVQFLKDDYTYLAVEI IRGDTIRYALLEI PSDKVPR FVNLPPEAPRRRKPMILLDNILRYCLDDIFKGFFDYDALNAYSMKMTRDAEYDLVHEMEA SLMELMSSSLKQRLTAEPVRFVYQRDMPNALVEVLREKLTISRYDS IVPGGRYHNFKDF I NFPNVGKANLVNKPLPRLRHIWFDKAQFRNGFDAIRERDVLLYYPYHTFEHVLELLRQAS FDPSVLAIKINIYRVAKDSRI IDSMIHAAHNGKKVTVVVELQARFDEEANIHWAKRLTEA GVHVIFSAPGLKIHAKLFLISRKENGEVVRYAHIGTGNFNEKTARLYTDYSLLTADARIT NEVRRVFNFIENPYRPVTFDYLMVSPQNSRRLLYEMVDREIANAQQGLPSGITLKLNNLV DKGLVDRLYAASS SGVPVNLLVRGMCSLI PNLEGISDNIRAIS IVDRYLEHDRVYIFENG GDKKVYLSSADWMTRNIDYRIEVATPLLDPRLKQRVLDI IDILFSDTVKARYIDKELSNR YVPRGNRRKVRAQLAIYDYIKSLEQPE

$>$ POAAC4

MDRFPRSDSIVQPRAGLQTYMAQVYGWMTVGLLLTAFVAWYAANSAAVMELLFTNRVFLI GLI IAQLALVIVLSAMIQKLSAGVTTMLFMLYSALTGLTLSS IF IVYTAAS IASTFVVTA GMFGAMSLYGYTTKRDLSGFGNMLFMALIGIVLASLVNFWLKSEALMWAVTYIGVIVFVG LTAYDTQKLKNMGEQIDTRDTSNLRKYS ILGALTLYLDF INLFLMLLR I FGNRR

$>$ P2 7457

ARNNTITLYDLQLESGCTISPYVWRTKYALKHKGFDIDIVPGGFTGILERTGGRSERVPV IVDDGEWVLDSWVIAEYLDEKYPDRPMLFEGPTQKNLMKFLDNWLWSTAVGPWFRCYILD YHDLSLPQDRDYVRWSREQWFLGGQRLEDVQAGREDRLPLVPPTLEPFRRILAETKWLGG DQPNFADYSALAVFLWTASVARTPPLTEDDPLRDWLDRGFDLFDGLGRHPGMNPLFGLKL REGDPEPFVRQTGPAGAGGQALNKGPQTTKMPPRVAEKAD

$>$ POAAC6

MDRIVSSSHDRTSLLSTHKVLRNTYFLLSLTLAFSAITATASTVLMLPSPGLILTLVGMY GLMFLTYKTANKPTGI ISAFAFTGFLGYILGP ILNTYLSAGMGDVIAMALGGTALVFFCC SAYVLTTRKDMSFLGGMLMAGIVVVLIGMVAN I FLQLPALHLA ISAVF ILISSGAILFET SNIIHGGETNYIRATVSLYVSLYNIFVSLLSILGFASRD

$>$ P32135

MLTKKKWALFSLLTLCGGTIYKLPSLKDAFYI PMQEYFHLTNGQIGNAMSVNSFVTTVGF FLSIYFADKLPRRYTMSFSLIATGLLGVYLTTMPGYWGILFVWALFGVTCDMMNWPVLLK SVSRLGNSEQQGRLFGFFETGRGIVDTVVAFSALAVFTWFGSGLLGFKAGIWFYSLIVIA VGIIIFFVLNDKEEAP SVEVKKEDGASKNTSMTSVLKDKTIWLIAFNVFFVYAVYCGLTF FI PFLKNIYLLPVALVGAYGI INQYCLKMIGGP IGGMISDKILKSPSKYLCYTFI ISTAA LVLLIMLPHESMPVYLGMACTLGFGAIVFTQRAVFFAP IGEAKIAENKTGAAMALGSFIG YAPAMFCFSLYGYILDLNPGI IGYKIVFGIMACFAFSGAVVSVMLVKRISQRKKEMLAAE A

$>$ P0A6E6

AMTYHLDVVSAEQQMFSGLVEKIQVTGSEGELGIYPGHAPLLTAIKPGMIRIVKQHGHEE FIYLSGGILEVQPGNVTVLADTAIRGQDLDEARAMEAKRKAEEHISSSHGDVDYAQASAE LAKAIAQLRVIELTKKAM

$>Q 59659$

MADVNRGNRPLSPHLQVYRLPLAAITSIMTRITGHALVAGIVLITWWLVAAVTSPGAFAC ADWVVRSWLGFI ILTGSMWALWYHLLAGLRHLFYDAGYGLEIEQAHKSSQALIAGSVVLA VLTLIVFFVF

>P29131

MAQRDYVRRSQPAPSRRKKSTSRKKQRNLPAVSPAMVAIAAAVLVTFIGGLYFITHHKKE ESETLQSQKVTGNGLPPKPEERWRYIKELESRQPGVRAPTEPSAGGEVKTPEQLTPEQRQ LLEQMQADMRQQPTQLVEVPWNEQTPEQRQQTLQRQRQAQQLAEQQRLAQQSRTTEQSWQ QQTRTSQAAPVQAQPRQSKPASSQQPYQDLLQTPAHTTAQSKPQQAAPVARAADAPKPTA EKKDERRWMVQCGSFRGAEQAETVRAQLAFEGFDSKITTNNGWNRVVIGPVKGKENADST LNRLKMAGHTNCIRLAAGG

$>$ P4 2910

MHEITLLQGLSLAALVFVLGIDFWLEALFLFRPI IVCTLTGAILGDIQTGLITGGLTELA FAGLTPAGGVQPPNPIMAGLMTTVIAWSTGVDAKTAIGLGLPFSLLMQYVILFFYSAFSL FMTKADKCAKEADTAAFSRLNWTTMLIVASAYAVIAFLCTYLAQGAMQALVKAMPAWLTH GFEVAGGILPAVGFGLLLRVMFKAQYIPYLIAGFLFVCYIQVSNLLPVAVLGAGFAVYEF FNAKSRQQAQPQPVASKNEEEDYSNGI

$>$ P 97253 
SNPKDDYKIWLVINPSTWLPVIWIVATVVAIAVHAAVLAAPGFNWIALGAAKSAAK $>$ POABAO

MNLNATILGQAIAFVLFVLFCMKYVWPPLMAAIEKRQKEIADGLASAERAHKDLDLAKAS ATDQLKKAKAEAQVI IEQANKRRSQ I LDEAKAEAEQERTKIVAQAQAEIEAERKRAREEL RKQVAILAVAGAEKI IERSVDEAANSDIVDKLVAEL

$>$ P33011

MRADKSLSPFEIRVYRHYRIVHGTRVALAFLLTFLI IRLFTIPESTWPLVTMVVIMGPIS FWGNVVPRAFERIGGTVLGS I LGLIALQLELISLPLMLVWCAAAMFLCGWLALGKKPYQG LLIGVTLAIVVGSPTGEIDTALWRSGDVILGSLLAMLFTGIWPQRAF IHWRIQLAKSLTE YNRVYQSAFS PNLLERPRLESHLQKLLTDAVKMRGLIAPASKETRI PKS IYEGIQTINRN LVCMLELQINAYWATRPSHFVLLNAQKLRDTQHMMQQ I LLSLVHALYEGNPQPVFANTEK LNDAVEELRQLLNNHHDLKVVETPIYGYVWLNMETAHQLELLSNLICRAL

$>$ P33950

MKLKATLTLAAATLVLAACDQSSSANKSTAQTEAKSSSNNTFVYCTAKAPLGFSPALI IE GTSYNASSQQVYNRLVEFKKGSTDIEPALAESWEISDDGLSYTFHLRKGVKFHTTKEFTP TRDFNADDVVFSFQRQLDPNHPYHNVSKGTYPYFKAMKFPELLKSVEKVDDNTIRITLNK TDATFLASLGMDF IS IYSAEYADSMLKAGKPETLDSRPVGTGPFVFVDYKTDQAIQYVAH ENYWKGRTPLDRLVIS IVPDATTRYAKLQAGTCDLILF PNVADLAKMKTDPKVQLLEQKG LNVAYIAFNTEKAPFDNVKVRQALNYAVDKKAI IEAVYQGAGTSAKNPLPPTIWSYNDEI QDYPYDPEKAKQLLAEAGYPNGFETDFWIQPVIRASNPNPKRMAELIMADWAKIGVKTNP VTYEWADYRKRAKEGELTAGIFGWSGDNGDPDNFLSPLLGSSNI GNSNMARFNNSEFDAL LNEAIGLTNKEERAKLYKQAQVIVHNQAPWI PVAHSVGFAPLSPRVKGYVQSPFGYDAFY GVSVDGK

$>$ P0ABA6

MAGAKEIRSKIASVQNTQKITKAMEMVAASKMRKSQDRMAASRPYAETMRKVIGHLAHGN LEYKHPYLEDRDVKRVGYLVVSTDRGLCGGLNINLFKKLLAEMKTWTDKGVQCDLAMIGS KGVSFFNSVGGNVVAQVTGMGDNPSLSELIGPVKVMLQAYDEGRLDKLYIVSNKF INTMS QVPTISQLLPLPASDDDDLKHKSWDYLYEPDPKALLDTLLRRYVESQVYQGVVENLASEQ AARMVAMKAATDNGGSLI KELQLVYNKARQAS ITQELTEIVSGAAAV

$>$ P0A7C8

MTSLSRPRVEFISTILQTVLNLGLLCLGLILVVFLGKETVHLADVLFAPEQTSKYELVEG LVVYFLYFEFIALIVKYFQSGFHFPLRYFVYIGITAIVRLI IVDHKS PLDVLIYSAAILL LVITLWLCNSKRLKRE

$>$ POAAD8

MSTSDSIVSSQTKQSSWRKSDTTWTLGLFGTAIGAGVLFFPIRAGFGGLI PILLMLVLAY PIAFYCHRALARLCLSGSNPSGNITETVEEHFGKTGGVVITFLYFFAICPLLWIYGVTIT NTFMTFWENQLGFAPLNRGFVALFLLLLMAFVIWFGKDLMVKVMSYLVWPFIASLVLISL SLIPYWNSAVIDQVDLGSLSLTGHDGILITVWLGIS IMVFSFNFSPIVSSFVVSKREEYE KDFGRDFTERKCSQI ISRASMLMVAVVMFFAFSCLFTLSPANMAEAKAQNIPVLSYLANH FASMTGTKTTFAITLEYAAS I IALVAIFKSFFGHYLGTLEGLNGLVLKFGYKGDKTKVSL GKLNT ISMI F IMGSTWVVAYANPNI LDL I EAMGAP I IASLLCLLPMYA I RKAP SLAKYRG RLDNVFVTVIGLLTILNIVYKLF

$>$ POCOL 7

MLKRKKVKPITLRDVTI IDDGKLRKAITAASLGNAMEWFDFGVYGFVAYALGKVFFPGAD PSVQMVAALATFSVPFLIRPLGGLFFGMLGDKYGRQKILAITIVIMSISTFCIGLIPSYD TIGIWAP ILLLICKMAQGFSVGGEYTGAS IFVAEYSPDRKRGFMGSWLDFGS IAGFVLGA GVVVLISTIVGEANFLDWGWR I PFF IALPLGI I GLYLRHALEETPAFQQHVDKLEQGDRE GLQDGPKVSFKEIATKYWRSLLTCIGLVIATNVTYYMLLTYMPSYLSHNLHYSEDHGVLI I IAIMIGMLFVQPVMGLLSDRFGRRPFVLLGSVALFVLA I PAF I LINSNVIGLI FAGLLM LAVILNCFTGVMASTLPAMFPTHIRYSALAAAFNISVLVAGLTPTLAAWLVESSQNLMMP AYYLMVVAVVGLITGVTMKETANRPLKGATPAASDIQEAKEILVEHYDNIEQKIDDIDHE IADLQAKRTRLVQQHPRIDE

$>$ P38101

MTPTLLSAFWTYTLITAMTPGPNNILALSSATSHGFRQSTRVLAGMSLGFLIVMLLCAGI SFSLAVIDPAAVHLLSWAGAAYIVWLAWKIATSPTKEDGLQAKP ISFWASFALQFVNVKI ILYGVTALSTFVLPQTQALSWVVGVSVLLAMIGTFGNVCWALAGHLFQRLFRQYGRQLNI VLALLLVYCAVRIFY

$>$ POAEW1

MTGSMIVNNLAGLMIMLTSLFVISVKSYRLSCGFYACQSLVLVSIFATLSCLFAAEQLLIW 
SASAF ITKVLLVPLIMTYAARNI PQN I PEKALFGPAMMALLAALIVLLCAFVVQPVKLPM ATGLKPALAVALGHFLLGLLCIVSQRNI LRQI FGYCLMENGSHLVLALLAWRAPELVEIG IATDAIFAVIVMVLLARKIWRTHGTLDVNNLTALKG $>$ POAG3 8

MLMLFLTVAMVHIVALMSPGPDFFFVSQTAVSRSRKEAMMGVLGITCGVMVWAGIALLGL HLI IEKMAWLHTL IMVGGGLYLCWMGYQMLRGALKKEAVSAPAPQVELAKSGRSFLKGLL TNLANPKAI IYFGSVFSLFVGDNVGTTARWG I FALI IVETLAWFTVVASLFALPQMRRGY QRLAKWIDGFAGALFAGFGIHLIISR

$>$ POADZ7

MSFFISDAVAATGAPAQGSPMSLILMLVVFGLIFYFMILRPQQKRTKEHKKLMDS IAKGD EVLTNGGLVGRVTKVAENGYIAIALNDTTEVVIKRDFVAAVLPKGTMKAL

$>$ P33021

MDVMRSVLGMVVLLTIAFLLSVNKKKISLRTVGAALVLQVVIGGIMLWLPPGRWVAEKVA FGVHKVMAYSDAGSAFIFGSLVGPKMDTLFDGAGFIFGFRVLPAI I FVTALVS ILYYIGV MGILIRILGGIFQKALNISKIESFVAVTTIFLGQNEIPAIVKPFIDRLNRNELFTAICSG MAS IAGSTMIGYAALGVPVEYLLAASLMA I PGGILFARLLSPATESSQVSFNNLSFTETP PKS I IEAAATGAMTGLKIAAGVATVVMAFVA I IAL ING I I GGVGGWFGFEHASLES ILGY LLAPLAWVMGVDWSDANLAGSLIGQKLA INEFVAYLNFSPYLQTAGTLDAKTVAI I SFAL CGFANFGS IGVVVGAFSAVAPHRAPEIAQLGLRALAAATLSNLMSATIAGFF IGLA

$>$ P09833

MLELNFSQTLGNHCLTINETLPANGITAIFGVSGAGKTSLINAISGLTRPQKGRIVLNGR VLNDAEKGICLTPEKRRVGYVFQDARLFPHYKVRGNLRYGMSKSMVDQFDKLVALLGIEP LLDRLPGSLSGGEKQRVAIGRALLTAPELLLLDEPLASLDI PRKRELLPYLQRLTREINI PMLYVSHSLDEILHLADRVMVLENGQVKAFGALEEVWGSSVMNPWLPKEQQSS ILKVTVL EHHPHYAMTALALGDQHLWVNKLDEPLQAALR IR IQASDVSLVLQP PQQTS IRNVLRAKV VNSYDDNGQVEVELEVGGKTLWARISPWARDELAIKPGLWLYAQIKSVSI

$>$ POABB 8

MFKEIFTRLIRHLPSRLVHRDPLPGAQQTVNTVVPPSLSAHCLKMAVMPEEELWKTFDTH PEGLNQAEVESAREQHGENKLPAQQPSPWWVHLWVCYRNPFNILLTILGAISYATEDLFA AGVIALMVAISTLLNFIQEARSTKAADALKAMVSNTATVLRVINDKGENGWLEI P IDQLV PGDI I KLAAGDMI PADLRILQARDLFVAQASLTGESLPVEKAATTRQPEHSNPLECDTLC FMGTTVVSGTAQAMVIATGANTWFGQLAGRVSEQESEPNAFQQGISRVSMLLIRFMLVMA PVVLLINGYTKGDWWEAALFALSVAVGLTPEMLPMIVTSTLARGAVKLSKQKVIVKHLDA IQNFGAMD I LCTDKTGTLTQDKIVLENHTDISGKTSERVLHSAWLNSHYQTGLKNLLDTA VLEGTDEESARSLASRWQKIDEI PFDFERRRMSVVVAENTEHHQLVCKGALQEILNVCSQ VRHNGEIVPLDDIMLRKIKRVTDTLNRQGLRVVAVATKYLPAREGDYQRADESDLILEGY IAFLDPPKETTAPALKALKASGITVKILTGDSELVAAKVCHEVGLDAGEVVIGSDIETLS DDELANLAQRTTLFARLTPMHKERIVTLLKREGHVVGFMGDGINDAPALRAADIGISVDG AVDIAREAADI I LLEKSLMVLEEGVIEGRRTFANMLKYIKMTASSNFGNVFSVLVASAFL PFLPMLPLHLLIQNLLYDVSQVAI PFDNVDDEQIQKPQRWNPADLGRFMIFFGPISSIFD ILTFCLMWWVFHANTPETQTLFQSGWFVVGLLSQTLIVHMIRTRRVPFIQSCASWPLMIM TVIVMIVGIALPFSPLASYLQLQALPLSYFPWLVAILAGYMTLTQLVKGFYSRRYGWQ $>$ POAAE5

MEKKLGLSALTALVLSSMLGAGVFSLPQNMAAVASPAALLIGWGITGAGILLLAFAMLIL TRIRPELDGGIFTYAREGFGELIGFCSAWGYWLCAVIANVSYLVIVFSALSFFTDTPELR LFGDGNTWQS IVGASALLWIVHFLILRGVQTAAS INLVATLAKLLPLGLFVVLAMMMFKL DTFKLDFTGLALGVPVWEQVKNTMLITLWVF IGVEGAVVVSARARNKRDVGKATLLAVLS ALGVYLLVTLLSLGVVARPELAEIRNP SMAGLMVEMMGPWGEI I IAAGLIVSVCGAYLSW TIMAAEVPFLAATHKAFPR I FARQNAQAAPSASLWLTNICVQI CLVLIWLTGSDYNTLLT IASEMILVPYFLVGAFLLKIATRPLHKAVGVGACIYGLWLLYASGPMHLLLSVVLYAPGL LVFLYARKTHTHDNVLNRQEMVLIGMLLIASVPATWMLVG

$>$ P06282

MKKAGLLFLVMIVIAVVAAGIGYWKLTGEESDTLRKIVLEECLPNQQQNQNPSPCAEVKP NAGYVVLKDLNGPLQYLLMPTYRINGTESPLLTDPSTPNFFWLAWQARDFMSKKYGQPVP DRAVSLAINSRTGRTQNHFHIHISCIRPDVRKQLDNNLAN ISSRWLPLPGGLRGHEYLAR RVTESELVQRSPFMMLAEEVPEAREHMGRYGLAMVRQSDNSFVLLATQRNLLTLNRASAE EIQDHQCEIL

$>$ P14376

MKYLASFRTTLKASRYMFRALALVLWLLIAFSSVFYIVNALHQRESEIRQEFNLSSDQAQ 
RFIQRTSDVMKELKYIAENRLSAENGVLSPRGRETQADVPAFEPLFADSDCSAMSNTWRG SLESLAWFMRYWRDNFSAAYDLNRVFLIGSDNLCMANFGLRDMPVERDTALKALHERINK YRNAPQDDSGSNLYWISEGPRPGVGYFYALTPVYLANRLQALLGVEQTIRMENFFLPGTL PMGVTILDENGHTLISLTGPESKIKGDPRWMQERSWFGYTEGFRELVLKKNLPPSSLSIV YSVPVDKVLERIRMLILNA I LLNVLAGAALFTLARMYERR IFI PAESDALRLEEHEQFNR KIVASAPVGICILRTADGVNILSNELAHTYLNMLTHEDRQRLTQI I GQQVNFVDVLTSN NTNLQISFVHSRYRNENVAICVLVDVSSRVKMEESLQEMAQAAEQASQSKSMFLATVSHE LRTPLYGI I GNLDLLQTKELPKGVDRLVTAMNNSSSLLLKI ISDILDFSKIESEQLKIEP REFSPREVMNHITANYLPLVVRKQLGLYCFIEPDVPVALNGDPMRLQQVISNLLSNAIKF TDTGCIVLHVRADGDYLSIRVRDTGVGI PAKEVVRLFDPFFQVGTGVQRNFQGTGLGLAI CEKLISMMDGDISVDSEPGMGSQFTVRI PLYGAQYPQKKGVEGLSGKRCWLAVRNASLCQ FLETSLQRSGIVVTTYEGQEPTPEDVLITDEVVSKKWQGRAVVTFCRRHIGI PLEKAPGE WVHSVAAPHELPALLARIYLIEMESDDPANALPSTDKAVSDNDDMMI LVVDDHPINRRLL ADQLGSLGYQCKTANDGVDALNVLSKNHIDIVLSDVNMPNMDGYRLTQRIRQLGLTLPVI GVTANALAEEKQRCLESGMDSCLSKPVTLDVIKQTLTLYAERVRKSRDS

$>$ POAFUO

MGAYLIRRLLLVIPTLWAI ITINFF IVQIAPGGPVDQA IAA IEFGNAGVLPGAGGEGVRA SHAQTGVGNISDSNYRGGRGLDPEVIAEITHRYGFDKP IHERYFKMLWDYIRFDFGDSLF RSASVLTLIKDSLPVS ITLGLWSTLI IYLVS I PLGIRKAVYNGSRFDVWSSAF I I IGYAI PAFLFAILLIVFFAGGSYFDLFPLRGLVSANFDSLPWYQKITDYLWHITLPVLATVIGGF AALTMLTKNSFLDEVRKQYVVTARAKGVSEKNILWKHVFRNAMLLVIAGFPATFISMFFT GSLLIEVMFSLNGLGLLGYEATVSRDYPVMFGTLYIFTLIGLLLNIVSDISYTLVDPRID FEGR

$>Q 47377$

MIWLTLVFASLLSVAGQLCQKQATCFVAINKRRKHIVLWLGLALACLGLAMVLWLLVLQN VPVGIAYPMLSLNFVWVTLAAVKLWHEPVSPRHWCGVAFI IGGIVILGSTV

$>$ P04683

MDRRVVITGLGGLCGLGTDASS IWTEMREGRSAIGP ISNSE IHELKGMIGTEI KVLPQHD IDRKQLISMDRFSLLAVLAAKQAMLQAGLSCNEGNAHRFGATVGVGFGGWDATEKAYRTL LLGGATRTELFTGVKAMPSAAACQVSMNLGLRGPVFGATSACASANHAIASAVDQI KLGR ADVMLAGGSDAPLVWIVLKAWEAMRVLAPDTCRPFSADRKGLVLGEGAGMAVLESYEHAA ARGATMLAEVAGIGLSADAYHIAAPAVHGPEAAMRACLVDASLNAEDVDYLNAHGTGTKA NDQIETTAIKRVFGDHARSMS ISSTKSTHAHCLGAASALEMIACVMAIQEGVVPPTANYR EPDPDCDLDVTPNVPRERKVRVAMSNAFAMGGMNAVLAFK

$>$ Q3 J6C1

MILGPDGILNRDTRGDWVRLRTLILLRWMAVAGQLAAIVVTDWYLGVRLPMGLCFMAVGA SVIANVIATFVFPQNRRLTEFQALMILLFDLTQLSFLLFLTGGLTNPFALLILAPVTISA LALELRTTVILGAIAIGLLTFTAYFHLPLILADGSSLSVPRMFEFGFWLAIVIGILFLGL YSRRVAIEIRSMSDALLATQMALDREQKLTDLGGVVAAAAHELGTPLATI KLVSSELAEE LSEQPALRDDAELIREQADRCRDILRSMGRAGKDDLQMRQAPLGEVLREAAEPHVGRGKR VEFDLYPSRGGDERQPVILRRPEVIHGLRNLIQNAVDFARSTVWIDGEWTGDRIAIRIVD DGEGYPPAI IGRIGDPFVRQRRAEESQSRRPGYEGMGLGLFIAKTLLERSGAELSFANAA DPFLRSHERPERCGAIVEVIWPVDRLVVVRNAPLGENVLIQT

$>$ P03959

MAAQGFLLIATFLLVLMVLARPLGSGLARLINDI PLPGTTGVERVLFRALGVSDREMNWK QYLCAILGLNMLGLAVLFFMLLGQHYLPLNPQQLPGLSWDLALNTAVSFVTNTNWQSYSG ETTLSYFSQMAGLTVQNFLSAASGIAVIFALIRAFTRQSMSTLGNAWVDLLRITLWVLVP VALLIALFFIQQGALQNFLPYQAVNTVEGAQQLLPMGPVASQEAI KMLGTNGGGFFNANS SHPFENPTALTNFVQMLAIFLIPTALCFAFGEVMGDRRQGRMLLWAMSVIFVICVGVVMW AEVQGNPHLLALGTDSS INMEGKESRFGVLVSSLFAVVTTAASCGAVIAMHDSFTALGGM VPMWLMQIGEVVFGGVGSGLYGMMLFVLLAVFIAGLMIGRTPEYLGKKIDVREMKLTALA ILVTPTLVLMGAALAMMTDAGRSAMLNPGPHGFSEVLYAVSSAANNNGSAFAGLSANSPF WNCLLAFCMFVGRFGVI I PVMAIAGSLVSKKSQAASSGTLPTHGPLFVGLLIGTVLLVGA LTFIPALALGPVAEYLS

$>$ P46136 MVGLIRGVSEGLGPVGGAAA IYSLSGLLLIFTVGFPRIRQI PKGYLLAGSLLFVSYEICL ALSLGYAATHHQA I EVGMVNYLWPSLTILFA I LFNGQKTNWLIVPGLLLALVGVCWVLGG DNGLHYDEI INNITTSPLSYFLAF IGAFIWAAYCTVTNKYARGFNGITVFVLLTGASLWV YYFLTPQPEMIFSTPVMIKLISAAFTLGFAYAAWNVGILHGNVTIMAVGSYFTPVLSSAL 
AAVLLSAPLSFSFWQGALMVCGGSLLCWLATRRG

$>$ P46139

MMDNDNSLNKRPTFKRALRNISMTS IFITMMLIWLLLSVTSVLTLKQYAQKNLALTAATM TYSLEAAVVFADGPAATETLAALGQQGQFSTAEVRDKQQNILASWHYTRKDPGDTFSNF I SHWLFPAPI IQPIRHNGETIGEVRLTARDSS ISHF IWFSLAVLTGCILLASGIAITLTRH LHNGLVEALKNITDVVHDVRSNRNFSRRVSEERIAEFHRFALDFNSLLDEMEEWQLRLQA KNAQLLRTALHDPLTGLANRAAFRSGINTLMNNSDARKTSALLFLDGDNFKYINDTWGHA TGDRVLIEIAKRLAEFGGLRHKAYRLGGDEFAMVLYDVQSESEVQQICSALTQI FNLPFD LHNGHQTTMTLSIGYAMTIEHASAEKLQELADHNMYQAKHQRAEKLVR

$>052043$

MESLQQLQQQLGLMAWPLF ICSALTVMLLAERLFQVLLSLTVGKGA IRHALQATSPKNPK QLAELTEHFASKRPVLYRGVAMLLAHHQFDKSLREDAAGIWLQEQRHQFNSGLRLLTLIG VISPLLGLLGTVLGLIEMFKGVAATTGS ITPNVLADGLGVAMYTTAAGLLIAVPAVAGAQ LLSLWADRTMAKLEHTLNYVNLWLEGMTLHADASLTVVTPQEATTENL

$>$ P76221

MMMMQSRKIWYYRITLI ILLFAMLLAWALLPGVHEF INRSVAAFAAVDQQGIERFIQSYG ALAAVVSFLLMILQAIAAPLPAFLITFANASLFGAFWGGLLSWTSSMAGAALCFF IARVM GREVVEKLTGKTVLDSMDGFFTRYGKHTILVCRLLPFVPFDPISYAAGLTSIRFRSFFIA TGLGQLPATIVYSWAGSMLTGGTFWFVTGLFILFALTVVI FMAKKIWLERQKRNA

$>$ P76224

MATPLRYALIFLLWAMVAVIYAPLI PAALTLISPALSLTHWQALFADPQLPQALLATLVS TTIAAVGALLIALLVIVALWPGPKWQRMCARLPWLLAI PHVAFATSALLLFADGGLLYDY FPYFTPPMDRFGIGLGLTLAVKESAFLLWILAAVLSEKWLLQQVIVLDSLGYSRWQCLNW LLLPSVAPALAMAMLA I VAWSLSVVDVA I I LGPGNPPTLAVISWQWLTQGDIDQQTKGAL ASLLLMLLLAAYVLLSYLLWRSWRRTI PRVDGVRKPATPLLPGNTLAIFLPLTGVLCVVL LAILADQSTINSEAL INSLTMGLVATF IALLLLLLWLEWGPQRRQLWLWLPILLPALPLV AGQYTLALWLKLDGSWTAVVWGHLLWVMPWMLFILQPAWQRIDSRLILIAQTLGWSRAKI FFYVKCPLMLRPVLIAFAVGFAVGIAQYMPTLWLGAGRFPTLTTEAVALSSGGSNGILAA QALWQLLLPLIIFALTALVAKWVGYVRQGLR

$>$ POABC9

MTDLSHSREKDKINPVVFYTSAGLILLFSLTTILFRDFSALWIGRTLDWVSKTFGWYYLL AATLYIVFVVCIACSRFGSVKLGPEQSKPEFSLLSWAAMLFAAGIGIDLMFFSVAEPVTQ YMQPPEGAGQTIEAARQAMVWTLFHYGLTGWSMYALMGMALGYFSYRYNLPLTIRSALYP IFGKRINGPIGHSVDIAAVIGTIFGIATTLGIGVVQLNYGLSVLFDI PDSMAAKAALIAL SVIIATISVTSGVDKGIRVLSELNVALALGLILFVLFMGDTSFLLNALVLNVGDYVNRFM GMTLNSFAFDRPVEWMNNWTLFFWAWWVAWSPFVGLFLARISRGRTIRQFVLGTLI I PFT FTLLWLSVFGNSALYEI IHGGAAFAEEAMVHPERGFYSLLAQYPAFTFSASVATITGLLF YVTSADSGALVLGNFTSQLKDINSDAPGWLRVFWSVA IGLLTLGMLMTNGISALQNTTVI MGLPFSFVIFFVMAGLYKSLKVEDYRRESANRDTAPRPLGLQDRLSWKKRLSRLMNYPGT RYTKQMMETVCYPAMEEVAQELRLRGAYVELKSLPPEEGQQLGHLDLLVHMGEEQNFVYQ IWPQQYSVPGFTYRARSGKSTYYRLETFLLEGSQGNDLMDYSKEQVITDILDQYERHLNF IHLHREAPGHSVMFPDA

$>$ P32166

MTEQQISRTQAWLESLRPKTLPLAFAAI IVGTALAWWQGHFDPLVALLALITAGLLQILS NLANDYGDAVKGSDKPDRIGPLRGMQKGVITQQEMKRALI ITVVLICLSGLALVAVACHT LADFVGFLILGGLS I IAA ITYTVGNRPYGYIGLGDISVLVFFGWLSVMGSWYLQAHTLIP ALILPATACGLLATAVLNINNLRDINSDRENGKNTLVVRLGEVNARRYHACLLMGSLVCL ALFNLFSLHSLWGWLFLLAAPLLVKQARYVMREMDPVAMRPMLERTVKGALLTNLLFVLG IFLSQWAA

$>$ P76228

MKKVLLQNHPGSEKYSFNGWEI FNSNFERMI KENKAMLLCKWGFYLTCVVAVMFVFAAIT SNGLNERGLITAGCSFLYLLIMMGLIVRAGFKAKKEQLHYYQAKGIEPLS IEKLQALQLI APYRFYHKQWSETLEFWPRKPEPGKDTFQYHVLPFDSIDI ISKRRESLEDQWGIEDSESY CALMEHFLSGDHGANTFKANMEEAPEQVIALLNKFAVFPSDYISDCANHSSGKSSAKLIW AAELSWMIS ISSTAFQNGTIEEELAWHYIMLASRKAHELFESEEDYQKNSQMGFLYWHIC CYRRKLTDAELEACYRYDKQFWEHYSKKCRWPIRNVPWGASSVKYS

$>Q 56348$

MIDSAKETDRPKHRKRDEVIAFLILAVVIWPILSVAIVGGYGFLVWMSQI IFGPPGPMH $>Q 51330$ 
NNPQTGQSTGLLGNRWFYLVLAVLLMCMI SGVQYSWTLYANPVKDNLGVSLAAVQTAFTL SQVIQAGSQPGGGYFVDKFGPRI PLMFGGAMVLAGWTFMGMVDSVPALYALYTLAGAGVG IVYGIAMNTANRWFPDKRGLASGFTAAGYGLGVLPFLPLISSVLKVEGVGAAFMYTGLIM GILIILIAFVIRFPGQQGAKKQIVVTDKDFNSGEMLRTPQFWVLWTAFFSVNFGGLLLVA NSVPYGRSLGLAAGVLTIGVSIQNLFNGGCRPFWGFVSDKIGRYKTMSVVFGINAVVLAL FPTIAALGDVAF IAMLA IAFFTWGGSYALFPSTNSD I FGTAYSARNYGFFWAAKATAS IF GGGLGAAIATNFGWNTAFLITAITSFIAFALATFVI PRMGRPVKKMVKLSPEEKAVH $>$ POAF 80 MHILDSLLAFSAYFFIGVAMVI IFLF IYSKITPHNEWQLI KNNNTAASLAFSGTLLGYVI PLSSAAINAVS I PDYFAWGGIALVIQLLVFAGVRLYMPALSEKI INHNTAAGMFMGTAAL AGGIFNAACMTW

$>$ P46141 MRNSHNITLTNNDSLTEDEETTWSLPGAVVGFISWLFALAMPMLIYGSNTLFFFIYTWPF FLALMPVAVVVGIALHSLMDGKLRYS IVFTLVTVGIMFGALFMWLLG

$>$ P38055

MEQYDQIGARLDRLPLARFHYRIFGI ISFSLLLTGFLSYSGNVVLAKLVSNGWSNNFLNA AFTSALMFGYFIGSLTGGF I GDYFGRRRAFR INLLIVGIAATGAAFVPDMYWLIFFRFLM GTGMGALIMVGYASFTEFI PATVRGKWSARLSFVGNWS PMLSAA IGVVVIAFFSWRIMFL LGGIGILLAWFLSGKYF IES PRWLAGKGQIAGAECQLREVEQQIEREKS IRLPPLTSYQS NSKVKVI KGTFWLLFKGEMLRRTLVAITVLIAMNISLYTITVWI PTIFVNSGIDVDKS IL MTAVIMIGAPVGIFIAALI IDHFPRRLFGSTLLI I IAVLGYIYSIQTTEWAI LIYGLVMI FFLYMYVCFASAVYIPELWPTHLRLRGSGFVNAVGRIVAVFTPYGVAALLTHYGS ITVFM VLGVMLLLCALVLSIFGIETRKVSLEEISE

$>$ P37180

MSHDPQPLGGKI ISKPVMI FGPLIVICMLLIVKRLVFGLGSVSDLNGGFPWGVWIAFDLL IGTGFACGGWALAWAVYVFNRGQYHPLVRPALLASLFGYSLGGLS ITIDVGRYWNLPYFY I PGHFNVNSVLFETAVCMTIYIGVMALEFAPALFERLGWKVSLQRLNKVMFF I IALGALL PTMHQSSMGSLMISAGYKVHPLWQSYEMLPLFSLLTAF IMGFS IVIFEGSLVQAGLRGNG PDEKSLFVKLTNTISVLLAIFIVLRFGELIYRDKLSLAFAGDFYSVMFWIEVLLMLFPLV VLRVAKLRNDSRMLFLSALSALLGCATWRLTYSLVAFNPGGGYAYFPTWEELLISIGFVA IEICAYIVLIRLLPILPPLKQNDHNRHEASKA

$>$ P37327

MDNDKIDQHSDEIEVESEEKERGKKIEIDEDRLPSRAMAIHEHIRQDGEKELERDAMALL WSAIAAGLSMGASLLAKGI FQVELEGVPGSFLLENLGYTFGF I IVIMARQQLFTENTVTA VLPVMQKPTMSNVGLLIRLWGVVLLGNILGTGIAAWAFEYMP I FNEETRDAFVKIGMDVM KNTPSEMFANA I I SGWL IATMVWMFPAAGAAKIVVI I LMTWLIALGDTTH IVVGSVEILY LVFNGTLHWSDF IWPFALPTLAGNICGGTF IFALMSHAQI RNDMSNKRKAEARQKAERAE NI KKNYKNPA

$>$ POA1W8

MTHQLKSRDI IALGFMTFALFVGAGNI I FPPMVGLQAGEHVWTAAIGFLITAVGLPVLTV VALAKVGGGVDSLSTPIGKVAGLLLATVCYLAVGPLFATPRTATVSFEVGIAPLTGDSAM PLLIYSVVYFAIVILVSLYPGKLLDTVGNFLAPLKI IALVILSVAA IVWPAGP I SNALDA YQNAAFSNGFVNGYLTMDTLGAMVFGIVIVNAARSRGVTEARLLTRYTVWAGLMAGVGLT LLYLALFRLGSDSATLVDQSANGAA I LHAYVQHTFGGAGSFLLAALI F IACLVTAVGLTC ACAEFFAQYIPLSYRTLVFILGGFSMVVSNLGLSHLIQIS I PVLTAIYPPCIALVVLSFT RSWWHNSTRI IAPAMF I SLLFGILDGIKASAFGDMLPAWSQRLPLAEQGLAWLMPTVVMV ILAI IWDRAAGRQVTSSAH

$>$ POAEY8

MQNKLASGARLGRQALLFPLCLVLYEFSTYIGNDMIQPGMLAVVEQYQAGIDWVPTSMTA YLAGGMFLQWLLGPLSDRIGRRPVMLAGVVWF IVTCLAILLAQNIEQFTLLRFLQGISLC FIGAVGYAAIQESFEEAVCIKITALMANVALIAPLLGPLVGAAWIHVLPWEGMFVLFAAL AAISFFGLQRAMPETATRIGEKLSLKELGRDYKLVLKNGRFVAGALALGFVSLPLLAWIA QSPI I I ITGEQLSSYEYGLLQVPI FGALIAGNLLLARLTSRRTVRSLI IMGGWP IMIGLL VAAAATVISSHAYLWMTAGLS IYAFGIGLANAGLVRLTLFASDMSKGTVSAAMGMLQMLI FTVGIEISKHAWLNGGNGLFNLFNLVNGILWLSLMVIFLKDKQMGNSHEG

$>$ P52636

MAENKRTRWQRRPGTTGGKLPWNDWRNATTWRKATQLLLLAMNIYIAITFWYWVRYYETA SSTTFVARPGGIEGWLPIAGLMNLKYSLVTGQLPSVHAAAMLLLVAFIVISLLLKKAFCS WLCPVGTLSELIGDLGNKLFGRQCVLPRWLDI PLRGVKYLLLSFFIYIALLMPAQAIHYF 
MLSPYSVVMDVKMLDFFRHMGTATLISVTVLLIASLFIRHAWCRYLCPYGALMGVVSLLS PFKIRRNAESCIDCGKCAKNCPSRI PVDKLIQVRTVECTGCMTCVESCPVASTLTFSLQK PAANKKAFALSGWLMTLLVLGIMFAVIGYAMYAGVWQSPVPEELYRRLI PQAPMIGH $>$ P18814

MAMVQPKSQKLRLFTTHLLLLIFIAAIMFPLLMVIAISLREGNFATGSLIPDTISWEHWR LALGFSVEHADGRVTPPPFPVLLWLWNS I KVAGITAIGIVALSTTCAYAFARMRFPGKAT LLKGMLIFQMFPAVLSLVALYALFDRLGQYLPFVGLNTHGGVIFAYMGGIALHVWTIKGY FETIDGSLEEAAALDGATPWQAFRLVLVPLSVPILAVVF ILSFIAAITEVPVASLLLRDV NSYTLAVGMQQYLNPQNYLWGDFAAAAVLSAI P ITVVFLLAQRWLVNGLTAGGVKG $>$ P31440

MI I ITEPLLSFVLQKQGIKSPPMDKKMNNDNTDYVSNESGTLSRLFKLPQHGTTVRTELI AGMTTFLTMVYIVFVNPQILGAAQMDPKVVFVTTCLIAGIGSIAMGIFANLPVALAPAMG LNAFFAFVVVGAMGISWQTGMGA IFWGAVGLFLLTLFRIRYWMISNIPLSLRIGITSGIG LFIALMGLKNTGVIVANKDTLVMIGDLSSHGVLLGILGFFI ITVLSSRHFHAAVLVS IVV TSCCGLFFGDVHFSGVYS I PPDISGVIGEVDLSGALTLELAGI IFSFMLINLFDSSGTLI GVTDKAGLIDGNGKFPNMNKALYVDSVSSVAGAFIGTSSVTAYIESTSGVAVGGRTGLTA VVVGVMFLLVMFFSPLVAIVPPYATAGALIFVGVLMTSSLARVNWDDFTESVPAFITTVM MPFTFSITEGIALGFMSYCIMKVCTGRWRDLNLCVVVVAALFALKI ILVD

$>$ P09852

MTATDRTPPPLKWLCLGNRDANDGFELFAHGIYARNGALVGSKLSLRERRQRVDLSAFLS GAPPLLAEAAVKHLLARLLCVHRHNTDLELLGKNF I PLHASSLGNAGVCERILASARQLQ QHQVELCLLLAIDEQEPASAEYLTSLARLRDSGVRIALHPQR IDTDARQCFAEVDAGLCD YLGLDARLLAPGPLTRNLRQRKS IEYLNRLLVAQDIQMLCLNVDNEELHQQANALPFAFR HGRHYSEPFQAWPFSSPAC

$>$ P21822

MFNRIKVVTSLLLVLVLFGALQLISGGLFFSSLKGDKENFTVLQTIRQQQLLLSESRVDL LQARNSLNRAGIRYMMDTNKIGSGATIDELLAKAEKEKLARAERNYTAYEKI PQDPRQDP QATEKLKQQYGILYGALSELIQLLGGGCKINAFFDQPTQKYQDDFEQTYNAYLQQNGKLY QIAVDASNSSYSSAIWTLIVVIIVVLAAIVGVWMGIHHILVRPLNRMIEHIKRIASGDLT QPIPVTSRNEIGVLAASLKHMQNELIETVSGVRQGADRS IDGASESAAGNNDLSSRTEQQ AAALEETAASMEQLTATVKQNAENARQASQLALSASETAQKGGKVVANVVETMHDIASSS QKIADITGVIDVIAFQTNILALNAAVEAARAGEQGRAFAVVAGEVRNLAQRSAQAAKEIK ALIEDSVNRVDMGSVLVESAGDTMGDIVNAVTRVTDIMGEIASASDEQSRGIDQVGQAVR EMDRVTQQNASLVEESASAAALEEQASLLTQSVAVFRLKSEGQEEYKAPVSNKTAPAAIA THKKTSASDYQDNWETF

$>$ P31446

MGI IAQNKISSLGMLFGA IALMMGI I HFSFGPFSAPPPTFES IVADKTAEIKRGLLAGIK GEKITTVEKKEDVDVDKILNQSGIALAIAALLCAF IGGMRKENRWGIRGALVFGGGTLAF HTLLFGIGIVCSILLIFLIFSFLTGGSLV

$>$ P76090

MTLYQIKPLFQSLLRPTMFWLYKHHVTANHITLAALALSLLTGLLLMLAAQP ILFLLLPI VLFIRMALNALDGMLARECNQQTRLGA I LNETGDVISDIALYLPFLFLPESNASLVILML FCTILTEFCGLLAQTINGVRSYAGPFGKSDRALIFGLWGLAVAIYPQWMQWNNLLWS IAS ILLLWTAINRCRSVLLMSAE

$>$ P76236

MIQSTRISMGLFFKYFLSLTKIDPGQNYISLPSIKSSTHIALLFMVSMGTQKLKAQSFFI FSLLLTLILFCITTLYNENTNVKLI PQMNYLMVVVALFFLNAVIFLFMLMKYFTNKQILP TLILSLAFLSGLIYLVETIVI IHKP INGSTLIQTKSNDVS IFYIFRQLSF ICLTSLALFC YGKDNILDNNKKKTGILLLALI PFLVFPLLAHNLSSYNADYSLYVVDYCPDNHTATWGIN YTKILVCLWAFLLFFI IMRTRLASELWPLIALLCLASLCCNLLLLTLDEYNYTIWYISRG IEVSSKLFVVSFLIYNI FQELQLSSKLAVHDVLTNIYNRRYFFNSVESLLSRPVVKDFCV MLVDINQFKR INAQWGHRVGDKVLVS IVDI IQQS I RPDDI LARLEGEVFGLLFTELNSAQ AKI IAERMRKNVELLTGFSNRYDVPEQMTIS IGTVFSTGDTRNISLVMTEADKALREAKS EGGNKVIIHHI

$>$ P76169

MIKTTLLFFATALCEI IGCFLPWLWLKRNAS IWLLLPAGISLALFVWLLTLHPAASGRVY AAYGGVYVCTALMWLRVVDGVKLTLYDWTGALIALCGMLI IVAGWGRT

$>Q 47539$

MSVLINEKLHSRRLKWRWPLSRQVTLSIGTLAVLLTVWWTVATLQLISPLFLPPPQQVLE 
KLLTIAGPQGFMDATLWQHLAASLTRIMLALFAAVLFGI PVGIAMGLSPTVRGILDPI IE LYRPVPPLAYLPLMVIWFGIGETSKILLIYLAIFAPVAMSALAGVKSVQQVRIRAAQSLG ASRAQVLWFVILPGALPEILTGLRIGLGVGWSTLVAAELIAATRGLGFMVQSAGEFLATD VVLAGIAVIAI IAFLLELGLRALQRRLTPWHGEVQ

$>Q 03478$

MQKQEHVVFKRAKAHGHDEPHGGAWKVAFADFMIALMALFLVLWVMQVVDKEERKAIVAH LHSSSVFDKSYGNPFDTSQS ISPIDLAQDSSVPSKHNSNHVVSSYFQGDGDGPEINSLVP GTFDTQEQLAALAKVIEEMTAQINAQGNVNVTVTPQGLRIVLQDDYKQHMFSRGGAELTP FFEDLLLALAPLFEQVTNPLI ISGHTDA I PFKKRFGRQSNWALSASRADVARKTLVEGGM PDDRVMQVTGMSDRALLNPDEPDSSENRR IELF ILTTPAAKVLETLFGNQDDSELQKAKQ KAEFNQPVIRQEVIRYSADAEKQEAKIQAL

$>$ P13738

MKHLHRFFSSDASGGI ILI IAA I LAMIMANSGATSGWYHDFLETPVQLRVGSLEINKNML LWINDALMAVF FLLVGLEVKRELMQGSLASLRQAAF PVIAAIGGMIVPALLYLAFNYADP ITREGWA I PAATD IAFALGVLALLGSRVPLALKI FLMALA I IDDLGA I I I IALFYTNDLS MASLGVAAVAIAVLAVLNLCGARRTGVYILVGVVLWTAVLKSGVHATLAGVIVGFFIPLK EKHGRSPAKRLEHVLHPWVAYLILPLFAFANAGVSLQGVTLDGLTS I LPLGI IAGLLIGK PLGISLFCWLALRLKLAHLPEGTTYQQIMVVGILCGIGFTMSIF IASLAFGSVDPELINW AKLGILVGSISSAVIGYSWLRVRLRPSV

$>$ POAF98

MI I IRYLVRETLKSQLA I LF ILLLIFFCQKLVRILGAAVDGDI PANLVLSLLGLGVPEMA QLILPLSLFLGLLMTLGKLYTESEITVMHACGLSKAVLVKAAMILAVFTAIVAAVNVMWA GPWSSRHQDEVLAEAKANPGMAALAQGQFQQATNGSSVLFIESVDGSDFKDVFLAQIRPK GNARPSVVVADSGHLTQLRDGSQVVTLNQGTRFEGTALLRDFR ITDFQDYQA I IGHQAVA LDPNDTDQMDMRTLWNTDTDRARAELNWRITLVFTVFMMALMVVPLSVVNPRQGRVLSML PAMLLYLLFFLIQTSLKSNGGKGKLDPTLWMWTVNLIYLALAIVLNLWDTVPVRRLRASF SRKGAV

$>$ POA8D9

MSTPDNRSVNFFSLFRRGQHYSKTWPLEKRLAPVFVENRVIKMTRYAIRFMPPIAVFTLC WQIALGGQLGPAVATALFALSLPMQGLWWLGKRSVTPLPPAILNWFYEVRGKLQESGQVL APVEGKPDYQALADTLKRAFKQLDKTFLDD

$>$ P0A6J3

MVSSFTSAPRSGFYYFAQGWKLVSQPGIRRFVILPLLVNILLMGGAFWWLFTQLDVWIPT LMSYVPDWLQWLSYLLWPLAVISVLLVFGYFFSTIANWIAAPFNGLLAEQLEARLTGATP PDTGIFGIMKDVPRIMKREWQKFAWYLPRAIVLLILYFIPGIGQTVAPVLWFLFSAWMLA IQYCDYPFDNHKVPFKEMRTALRTRKITNMQFGALTSLFTMI PLLNLF IMPVAVCGATAM WVDCYRDKHA

$>$ P22 208

MSFNAKDMTQGGQIASMRIRMFSQIANIMLYCLFIFFWILIGLVLWVKISWQTFINGCIY WWCTSLEGMRDLIKSQPVYEIQYYGKTFRMNAAQVLHDKYMIWCGEQLWSAFVLASVVAL VICLITFFVVSWILGRQGKQQSENEVTGGRQLTDNPKDVARMLKKDGKDSDIRIGDLPI I RDSEIQNFCLHGTVGAGKSEVIRRLANYARQRGDMVVIYDRSGEFVKSYYDPSIDKILNP LDARCAAWDLWKECLTQPDFDNTANTLI PMGTKEDPFWQGSGRTIFAEAAYLMRNDPNRS YSKLVDTLLS IKIEKLRTFLRNSPAANLVEEKIEKTAIS IRAVLTNYVKAIRYLQGIEHN GDPFTIRDWMRGVREDQKNGWLF I SSNADTHASLKPVISMWLS IAIRGLLAMGENRNRRV WFFCDELPTLHKLPDLVEILPEARKFGGCYVFGIQSYAQLEDIYGEKAAATLFDVMNTRA FFRSPSHKIAEFAAGEIGEKEHLKASEQYSYGADPVRDGVSTGKDMERQTLVSYSDIQSL PDLTCYVTLPGPYPAVKLSLKYQARPKVAPEF I PRDINPEMENRLSAVLAAREAEGRQMA SLFEPEVASGEDVTQAEQPQQPQQPQQPQQPQQPQQPQQPQQPQQPVSSVINDKKSDAGV SVPAGGIEQELKMKPEEEMEQQLPPGISESGEVVDMAAYEAWQQENHPDIQQHMQRREEV NINVHRERGEDVEPGDDF

$>$ P0A6J5

MRVVILGSGVVGVASAWYLNQAGHEVTVIDREPGAALETSAANAGQISPGYAAPWAAPGV PLKAIKWMFQRHAPLAVRLDGTQFQLKWMWQMLRNCDTSHYMENKGRMVRLAEYSRDCLK ALRAETNIQYEGRQGGTLQLFRTEQQYENATRDIAVLEDAGVPYQLLESSRLAEVEPALA EVAHKLTGGLQLPNDETGDCQLFTQNLARMAEQAGVKFRFNTPVDQLLCDGEQIYGVKCG DEVIKADAYVMAFGSYSTAMLKGIVDI PVYPLKGYSLTIPIAQEDGAPVSTILDETYKIA ITRFDNRIRVGGMAEIVGFNTELLQPRRETLEMVVRDLYPRGGHVEQATFWTGLRPMTPD GTPVVGRTRFKNLWLNTGHGTLGWTMACGSGQLLSDLLSGRTPAI PYEDLSVARYSRGFT 
PSRPGHLHGAHS

$>$ P37340

MQKYISEARLLLALA I PVILAQIAQTAMGFVDTVMAGGYSATDMAAVAIGTS IWLPAILF GHGLLLALTPVIAQLNGSGRRERIAHQVRQGFWLAGFVSVLIMLVLWNAGYI IRSMENID PALADKAVGYLRALLWGAPGYLFFQVARNQCEGLAKTKPGMVMGFIGLLVNI PVNYIFIY GHFGMPELGGVGCGVATAAVYWVMFLAMVSYIKRARSMRDIRNEKGTAKPDPAVMKRLIQ LGLPIALALFFEVTLFAVVALLVSPLGIVDVAGHQIALNFS SLMFVLPMSLAAAVTIRVG YRLGQGSTLDAQTAARTGLMVGVCMATLTAIFTVSLREQIALLYNDNPEVVTLAAHLMLL AAVYQISDSIQVIGSGILRGYKDTRS IFYITFTAYWVLGLPSGYILALTDLVVEPMGPAG FWIGF I IGLTSAA IMMMLRMRFLQRLPSAI I LQRASR

$>$ P2 7650

MKYSKITTTLAALALPS IAHAHVGLHADGTLAGLNHPFSGLDHILAMVAVGFWASTLGGK AVWIVPSAFVIVMAGGGVLGIEGIALPMVETAIALTVAMLGLLVAFEVKI PTPVAAIVVG ICALFHGHVHGIELPTMSNATGYVAGFLAATVILHVLGIGLASLRFGKAGQVVARVAGGA VALAGAALLVG

$>$ P34009

MADELDDQAAMAQWASENPPGGGEGVNEFGDFSGGMGGWDDGGGDGASERILNQDEIDSL LGFDLSGDGSDDRTGIRAI INSALVSYERLPMLEIVFDRLVRLMTTSLRNFTSDNVEVSL DNISS IRFGDYLNS I PLPGILAVFRAEELDNYGLLTVDSNLIYS IVDVLLGGRRGTAAMR IEGRPYTTIERVLVQRMIDVVLHDLKSAFEPLHPVSFSLDRLETNPPFAAIARPANAAIL VKLRIDMEDRGGRIELLLPYATLEPIRKMLLQQFMGEKFGRDNIWEGHLATELWTTQMEV RAVLDEQQVPLSRVLNMQVGDTLMLNATSRQPGGAARRRI PLTRGRMGRRNHS IAVRAEA PVDRRKKAVQKLK

$>$ P23516

MFQLLAGVRMNSTGRPRAKI ILLYALLIAFNIGAWLCALAAFRDHPVLLGTALLAYGLGL RHAVDADHLAA IDNVTRKLMQDGRRPITAGLWFSLGHSSVVVLASVLIAVMATTLQERLD AFHEVGSVIGTLASALFLFAIAAINLVILRSAYRAFRRVRRGGIYVEEDFDLLFGNRGFL ARIFRPLFRFITRSWHMYPLGMLFALGFDTATEVALLGISTMEASRGVPIWS ILVFPALF TAGMALIDTIDS ILMCGAYAWAYAKPVRKLYYNMTITFVSAIVALIVGGIETLGLLADKF MLKGVFWNAVGALNENFCQLGFVI IGIFTVCWVVS IVVYRLRRYDDSEVR

$>$ P76180

MTTTTPQRIGGWLLGPLAWLLVALLSTTLALLLYTAALSSPQTFQTLGGQALTTQILWGV SFITAIALWYYTLWLTIAFFKRRRCVPKHYI IWLLISVLLAVKAFAFSPVEDGIAVRQLL FTLLATALIVPYFKRSSRVKATFVNP

$>$ P31468

MSVSRRVIHHGLYFAVLGPLIGVLFLVLYIFFAKEPLVLWVI IHPIFLLLSITTGAIPAL LTGVMVACLPEKIGSQKRYRCLAGGIGGVVITEIYCAVIVHIKGMASSELFENILSGDSL VVRI I PALLAGVVMSRI ITRLPGLDISCPETDSLS

$>Q 46755$

MTSFSTLLSVHLISIALSVGLLTLRFWLRYQKHPQAFARWTRIVPPVVDTLLLLSGIALM AKAH I LPFSGQAQWLTEKLFGV I IY IVLGF IALDYRRMHSQQARI IAFPLALVVLYI I IK LATTKVPLLG

$>$ P33218

MANWLNQLQSLLGQSSSSTSSSADQGLVKLLVPGALGGLAGLLVANKSARKLLTKYGTNA LLVGGGAVAGTVLWNKYKDKIRAAHQDEPQFGAQSTPLDERTARLILALVFAAKSDGHID AKERAAIDQQLRGAGVEEQGRVLIEQAIEQPLDPQRLATGVRNEEEALEIYFLSCAAIDI DHFMERSYLNALGDALKI PQDVRDGIERDLEQQKRTLAE

$>$ POABG4

MRLSLPRLKMPRLPGFS ILVWISTALKGWVMGSREKDTDSLIMYDRTLLWLTFGLAAIGF IMVTSASMPIGQRLTNDPFFFAKRDGVYL ILAF ILA I ITLRLPMEFWQRYSATMLLGS I I LLMIVLVVGSSVKGASRWIDLGLLRIOPAELTKLSLFCYIANYLVRKGDEVRNNLRGFLK PMGVILVLAVLLLAQPDLGTVVVLFVTTLAMLFLAGAKLWQF IA I I GMGISAVVLLILAE PYRIRRVTAFWNPWEDPFGSGYQLTQSLMAFGRGELWGQGLGNSVQKLEYLPEAHTDF IF A I IGEELGYVGVVLALLMVFFVAFRAMS I GRKALEIDHRFSGFLACS IGIWFSFQALVNV GAAAGMLPTKGLTLPLISYGGSSLLIMSTAIMMLLRIDYETRLEKAQAFVRGSR

$>$ P76334

MEKCDFYHI IVLSLNFPGYLKMEYGSTKMEERLSRSPGGKLALWAFYTWCGYFVWAMARY IWVMSRI PDAPVSGFESDLGSTAGKWLGALVGFLFMALVGALLGS IAWYTRPRPARSRRY $\mathrm{E}$ 
$>$ P18777

MGSGWHEWPLMIFTVFGQCVAGGFIVLALALLKGDLRAEAQQRVIACMFGLWVLMGIGF I ASMLHLGSPMRAFNSLNRVGASALSNEIASGS IFFAVGGIGWLLAMLKKLSPALRTLWLI VTMVLGVI FVWMMVRVYNS IDTVPTWYS IWTPMGFFLTMFMGGPLLGYLLLSLAGVDGWA MRLLPAISVLALVVSGVVSVMQGAELATIHSSVQQAAALVPDYGALMSWRIVLLAVALCL WIAPQLKGYQPAVPLLSVSFILLLAGELIGRGVFYGLHMTVGMAVAS

$>$ P76264

MNITATVLLAFGMSMDAFAAS IGKGATLHKPKFSEALRTGLIFGAVETLTPLIGWGMGML ASRFVLEWNHWIAFVLLIFLGGRMI IEGFRGADDEDEEPRRRHGFWLLVTTAIATSLDAM AVGVGLAFLQVNI IATALA IGCATL IMSTLGMMVGRF I GS I I GKKAE ILGGLVLIGIGVQ ILWTHFHG

$>$ P 67444

MGIYFTNSDDQIYFKRSEGMSDINHAGSDLIFELEDRPPFHQALVGAITHLLAIFVPMVT PALIVGAALQLSAETTAYLVSMAMIASGIGTWLQVNRYGIVGSGLLSIQSVNFSFVTVMI ALGSSMKSDGFHEELIMSSLLGVSFVGAFLVVGSSF I LPYLRRVITPTVSGIVVLMIGLS LIKVGI IDFGGGFAAKSSGTFGNYEHLGVGLLVLIVVIGFNCCRSPLLRMGGIAIGLCVG YIASLCLGMVDFSSMRNLPLITI PHPFKYGFSFSFHQFLVVGT I YLLSVLEAVGDITATA MVSRRPIQGEEYQSRLKGGVLADGLVSVIASAVGSLPLTTFAQNNGVIQMTGVASRYVGR TIAVMLVILGLFPMIGGFFTTI PSAVLGGAMTLMF SMIA IAGIRI I ITNGLKRRETLIVA TSLGLGLGVSYDPEIFKILPAS IYVLVENPI CAGGLTAILLNI I LPGGYRQENVLPGITS AEEMD

$>$ P76339

MKRLS ITVRLTLLF ILLLSVAGAGIVWTLYNGLASELKWRDDTTLINRTAQI KQLLIDGV NPDTLPVYFNRMMDVSQDI I I IHGDS INKIVNRTNVSDGMLNNI PASETISAAGIYRS I I NDTEIDALRINIDEVSPSLTVTVAKLASARHNMLEQYKINS I I ICIVAIVLCSVLSPLLI RTGLREIKKLSGVTEALNYNDSREPVEVSALPRELKPLGQALNKMHHALVKDFERLSQFA DDLAHELRTP INALLGQNQVTLSQTRS IAEYQKTIAGNIEELENISRLTENILFLARADK NNVLVKLDSLSLNKEVENLLDYLEYLSDEKEI CFKVECNQQ I FADKI LLQRMLSNLIVNA IRYSPEKSRIHITSFLDTNSYLNIDIASPGTKINEPEKLFRRFWRGDNSRHSVGQGLGLS LVKAIAELHGGSATYHYLNKHNVFRITLPQ

$>$ P08149

MLIKSEYKPRMLPKEEQVKKPMTSNGRISFVLMAMAVLFACLIARGLYLQTVTYNFLKEQ GDNRIVRTQALPATRGTVSDRNGAVLALSAPTESLFAVPKDMKEMPSAAQLERLSELVDV PVDVLRNKLEQKGKSF IWI KRQLDPKVAEEVKALGLENFVFEKELKRHYPMGNLFAHVIG FTDIDGKGQEGLELSLEDSLYGEDGAEVVLRDRQGNIVDSLDSPRNKAPQNGKDI ILSLD QRIQTLAYEELNKAVEYHQAKAGTVVVLDARTGEILALANTPAYDPNRPGRADSEQRRNR AVTDMIEPGSAIKPFVIAKALDAGKTDLNERLNTQPYKIGPSPVRDTHVYPSLDVRGIMQ KSSNVGTSKLSARFGAEEMYDFYHELGIGVRMHSGFPGETAGLLRNWRRWRPIEQATMSF GYGLQLSLLQLARAYTALTHDGVLLPLSFEKQAVAPQGKRIFKESTAREVRNLMVSVTEP GGTGTAGAVDGFDVGAKTGTARKFVNGRYADNKHVATF IGFAPAKNPRVIVAVTIDEPTA HGYYGGVVAGPPFKKIMGGSLNILGISPTKPLTAAAVKTPS

$>$ P22 729

MKPMHIAMALLSAAMFFVLAGVFMGVQLELDGTKLVVDTASDVRWQWVFIGTAVVFFFQL LRPAFQKGLKSVSGPKF ILPAIDGSTVKQKLFLVALLVLAVAWPFMVSRGTVDIATLTMI YI ILGLGLNVVVGLSGLLVLGYGGFYAIGAYTFALLNHYYGLGFWTCLPIAGLMAAAAGF LLGFPVLRLRGDYLAIVTLGFGEIVRILLLNNTEITGGPNGISQIPKPTLFGLEFSRTAR EGGWDTFSNFFGLKYDPSDRVIFLYLVALLLVVLSLFVINRLLRMPLGRAWEALREDEIA CRSLGLSPRRIKLTAFTISAAFAGFAGTLFAARQGFVSPESFTFAESAFVLAIVVLGGMG SQFAVILAAILLVVSRELMRDFNEYSMLMLGGLMVLMMIWRPQGLLPMTRPQLKLKNGAA KGEQA

$>Q 46909$

MNTSPVRMDDLPLNRFHCRIAALTFGAHLTDGYVLGVIGYAI IQLTPAMQLTPFMAGMIG GSALLGLFLGSLVLGWISDHIGRQKIFTFSFLLITLASFLQFFATTPEHLIGLRILIGIG LGGDYSVGHTLLAEFSPRRHRGILLGAFSVVWTVGYVLAS IAGHHF I SENPEAWRWLLAS AALPALLITLLRWGTPESPRWLLRQGRFAEAHA IVHRYFGPHVLLGDEVVTATHKHIKTL FSSRYWRRTAFNSVFFVCLVI PWFVIYTWLPTIAQTIGLEDALTASLMLNALLIVGALLG LVLTHLLAHRKFLLGSFLLLAATLVVMACLPSGSSLTLLLFVLFSTTISAVSNLVGILPA ESFPTDIRSLGVGFATAMSRLGAAVSTGLLPWVLAQWGMQVTLLLLATVLLVGFVVTWLW APETKALPLVAAGNVGGANEHSVSV 
$>$ POAG90

MLNRYPLWKYVMLIVVIVIGLLYALPNLFGEDPAVQITGARGVAASEQTLIQVQKTLQEE KITAKSVALEEGAILARFDSTDTQLRAREALMGVMGDKYVVALNLAPATPRWLAAIHAEP MKLGLDLRGGVHFLMEVDMDTALGKLQEQNIDSLRSDLREKGI PYTTVRKENNYGLSITF RDAKARDEAIAYLSKRHPDLVISSQGSNQLRAVMSDARLSEAREYAVQQNINILRNRVNQ LGVAEPVVQRQGADRIVVELPGIQDTARAKEILGATATLEFRLVNTNVDQAAAASGRVPG DSEVKQTREGQPVVLYKRVILTGDHITDSTSSQDEYNQPQVNISLDSAGGNIMSNFTKDN IGKPMATLFVEYKDSGKKDANGRAVLVKQEEVINIANIQSRLGNSFRITGINNPNEARQL SLLLRAGALIAPIQIVEERT IGPTLGMQNIEQGLEACLAGLLVS ILFMI I FYKKFGLIAT SALIANLILIVGIMSLLPGATLSMPGIAGIVLTLAVAVDANVLINERIKEELSNGRTVQQ AIDEGYRGAFSS I FDANITTLIKVI I LYAVGTGA I KGFAITTGIGVATSMFTAIVGTRAI VNLLYGGKRVKKLSI

$>$ P37433

MKGRLLQRLRQLSISNSLRGAFLTGALLTLIVSMVSLYSWHEQSSQVRYSLDEYFPRIHS AFLIEGNLNLAVDQLNEFLLAPNTTVRLQLRTQI IQHLDKIERLSQGLQLAERRQLAVIL QDSRTLLAELDNALYNMFLVREKVSELSARIDWLHDDFTTELNSLVQDFTWQQGTLLDQI EANQGDAAQYLQRSREVQNEQQQVYTLARIENQIVDDLRDRLNELKSGNNDGMLVETHIR YLENLKKTADENIRALDDWPSTITLRQTIDELLEIGMVKNKMPDTMRDYVAAQKALLDAS RAREATLGRFRTLLEAQLGSSHQQMQTFNQRLEQIVRVSGGLILVATLLALLLAWGLNHY FIRSRLVKRFTALNQAVVQIGLGRTDSTIPVYGRDELGRIARLLRHTLGQLNMQRRQLEQ EVAERKEIEADLRAMQDELIQTAKLAVVGQTMTTLAHEINQPLNALSMYLFTAGRAIEQG QSGQARNTLTKAEGLINRIDAI IRSLRQFTRRAELETPLYPVDLRQTFVAAWELLAMRHQ SRQGALSLPTDTVWVSGDEVRIQQVLVNVLANALDACSHDAVIAVTWQTQGEALEVYIAD NGPGWPVALLPSLLKPFTTSKAVGLGIGLS ISVSLMAQMKGDLRLASTLTRNACVVLQFS VTDVDDVE

$>$ P0AG96

MSANTEAQGSGRGLEAMKWVVVVALLLVAIVGNYLYRDIMLPLRALAVVILIAAAGGVAL LTTKGKATVAFAREARTEVRKVIWPTRQETLHTTLIVAAVTAVMSLILWGLDGILVRLVS FITGLRF

$>$ P36561

MSKLFWAMLSFITRLPVPRRWSQGLDFEHYSRGI ITFPLIGLLLGAISGLVFMVLQAWCG APLAALFSVLVLVLMTGGFHLDGLADTCDGVFSARSRDRMLEIMRDSRLGTHGGLALIFV VLAKILVLSELALRGES ILASLAAACAVSRGTAALLMYRHRYAREEGLGNVF IGKIDGRQ TCVTLGLAA I FAAVLLPGMHGVAAMVVTMVA I F I LGQLLKRTLGGQTGDTLGAA I ELGEL VFLLALL

$>$ P0AG99

MYEALLVVFLIVAIGLVGLIMLQQGKGADMGASFGAGASATLFGSSGSGNFMTRMTALLA TLFFI ISLVLGNINSNKTNKGSEWENLSAPAKTEQTQPAAPAKPTSDI PN

$>$ P46187

MI KEWATVVSWQNGQALVSCDVKASCSSCASRAGCGSRVLNKLGPQTTHTIVVPCDEPLV PGQKVELGIAEGSLLSSALLVYMS PLVGLFLIASLFQLLFASDVAALCGAILGGIGGFLI ARGYSRKFAARAEWQPI ILSVALPPGLVRFETSSEDASQ

$>$ P33226

MRKLWNALRRPSARWSVLALVAIGIVIGIALIVLPHVGIKVTSTTEFCVSCHSMQPVYEE YKQSVHFQNASGVRAECHDCHI PPDI PGMVKRKLEASNDIYQTF IAHS IDTPEKFEAKRA ELAEREWARMKENNSATCRSCHNYDAMDHAKQHPEAARQMKVAAKDNQSCIDCHKGIAHQ LPDMSSGFRKQFDELRASANDSGDTLYS IDIKPIYAAKGDKEASGSLLPASEVKVLKRDG DWLQIEITGWTESAGRQRVLTQFPGKRIFVAS IRGDVQQQVKTLEKTTVADTNTEWSKLQ ATAWMKKGDMVNDI KP IWAYADSLYNGTCNQCHGAPE IAHFDANGWIGTLNGMIGFTSLD KREERTLLKYLQMNASDTAGKAHGDKKEEK

$>$ POABH4

MASYRSQGRWVIWLSFLIALLLQIMPWPDNLIVFRPNWVLLILLYWILALPHRVNVGTGF VMGA I LDLISGSTLGVRVLAMS I IAYLVALKYQLFRNLALWQQALVVMLLSLVVDI IVFW AEFLVINVSFRPEVFWSSVVNGVLWPWIFLLMRKVRQQFAVQ

$>$ P76343

MRLTAKQVTWLKVCLHLAGLLPFLWLVWAINHGGLGADPVKDIQHFTGRTALKFLLATLL ITPLARYAKQPLLIRTRRLLGLWCFAWATLHLTSYALLELGVNNLALLGKELITRPYLTL GI ISWVILLALAFTSTQAMQRKLGKHWQQLHNFVYLVAILAPIHYLWSVKI ISPQPLIYA GLAVLLLALRYKKLRSLFNRLRKQVHNKLSV 
$>$ P31553

MKNEKRKTGIEPKVFFPPLI IVGILCWLTVRDLDAANVVINAVFSYVTNVWGWAFEWYMV VMLFGWFWLVFGPYAKKRLGNEPPEFSTASWI FMMFASCTSAAVLFWGS IEIYYY ISTPP FGLEPNSTGAKELGLAYSLFHWGPLPWATYSFLSVAFAYFFFVRKMEVIRPSSTLVPLVG EKHAKGLFGTIVDNFYLVALI FAMGTSLGLATPLVTECMQWLFGI PHTLQLDAI I ITCWI ILNA I CVACGLQKGVRIASDVRSYLSFLMLGWVF IVSGAS F IMNYFTDSVGMLLMYLPRM LFYTDPIAKGGFPQGWTVFYWAWWVIYAIQMS I FLAR I SRGRTVRELCFGMVLGLTASTW ILWTVLGSNTLLLIDKNI INI PNLIEQYGVARA I IETWAALPLSTATMWGFF ILCF IATV TLVNACSYTLAMSTCREVRDGEEPPLLVRIGWS I LVGI I GIVLLALGGLKPIQTAI IAGG CPLFFVNIMVTLSFIKDAKQ

$>$ P76417

MKTTAKLSFMMFVEWF IWGAWFVPLWLWLSKSGFSAGEIGWSYACTAIAAILSPILVGS I TDRFFSAQKVLAVLMFAGALLMYFAAQQTTFAGFFPLLLAYSLTYMPTIALTNSIAFANV PDVERDFPRIRVMGTIGWIASGLACGFLPQILGYADISPTNI PLLITAGSSALLGVFAFF LPDTPPKSTGKMDI KVMLGLDALILLRDKNFLVFFFCSFLFAMPLAFYYIFANGYLTEVG MKNATGWMTLGQFSEI FFMLALPFFTKRFGI KKVLLLGLVTAA IRYGFFIYGSADEYFTY ALLFLGILLHGVSYDFYYVTAY IYVDKKAPVHMRTAAQGLITLCCQGFGSLLGYRLGGVM MEKMFAYQEPVNGLTFNWSGMWTFGAVMIAI IAVLFMIFFRESDNEITAIKVDDRDIALT QGEVK

$>$ P76345

MNRFSKTQIYLHWITLLFVAITYAAMELRGWFPKGSSTYLLMRETHYNAGIFVWVLMFSR LI IKHRYSDPS IVPPPPAWQMKAASLMHIMLYITFLALPLLGIALMAYSGKSWSFLGFNV SPFVTPNSEI KALI KNIHETWANIGYFLIAAHAGAALFHHYIQKDNTLLRMMPRRK $>$ P0A6M2

MLRFLNQCSQGRGAWLLMAFTALALELTALWFQHVMLLKPCVLCIYERCALFGVLGAALI GAIAPKTPLRYVAMVIWLYSAFRGVQLTYEHTMLQLYPSPFATCDFMVRFPEWLPLDKWV PQVFVASGDCAERQWDFLGLEMPQWLLGIFIAYLIVAVLVVISQPFKAKKRDLFGR $>Q 46841$

MAGFNI KHWFADGAFRTI IRNSAWLGSSNVVSALLGLLALSCAGKGMTPAMFGVLVIVQS YAKS ISDF IKFQTWQLVVQYGTPALTNNNPQQFRNVVSFSFSLDIVSGAVAIVGGIALLP FLSHSLGLDDQSFWLAALYCTLI PSMAS STPTGILRAVDRFDLIAVQQATKPFLRAAGSV VAWYFDFGFAGFVIAWYVSNLVGGTMYWWFAARELRRRNIHNAFKLNLFESARYIKGAWS FVWSTNIAHS IWSARNSCSTVLVGIVLGPAAAGLFKIAMTFFDAAGTPAGLLGKSFYPEV MRLDPRTTRPWLLGVKSGLLAGGIGILVALAVLIVGKPLISLVFGVKYLEAYDLI

$>$ P76278

MLAFTWIALRFIHFTSLMLVFGFAMYGAWLAPLTIRRLLAKRFLRLQQHAAVWSLISATA MLAVQGGLMGTGWTDVFSPNIWQAVLQTQFGGIWLWQIVLALVTLIVALMQPRNMPRLLF MLTTAQF ILLAGVGHATLNEGVTAKIHQTNHA I HLICAAAWFGGLLPVLWCMQLI KGRWR HQAIQALMRFSWCGHFAVIGVLASGVLNALLITGFPPTLTTYWGQLLLLKAILVMIMVVI ALANRYVLVPRMRQDEDRAAPWFVWMTKLEWAIGAVVLVI ISLLATLEPF

$>$ P21865

MNNEPLRPDPDRLLEQTAAPHRGKLKVFFGACAGVGKTWAMLAEAQRLRAQGLDIVVGVV ETHGRKDTAAMLEGLAVLPLKRQAYRGRH ISEFDLDAALARRPAL I LMDELAHSNAPGSR HPKRWQDIEELLEAGIDVFTTVNVQHLESLNDVVSGVTGIQVRETVPDPFFDAADDVVLV DLPPDDLRQRLKEGKVYIAGQAERAIEHFFRKGNLIALRELALRRTADRVDEQMRAWRGH PGEEKVWHTRDAILLCIGHNTGSEKLVRAAARLASRLGSVWHAVYVETPALHRLPEKKRR AILSALRLAQELGAETATLSDPAEEKAVVRYAREHNLGKI ILGRPASRRWWRRETFADRL ARIAPDLDQVLVALDEPPARTINNAPDNRSFKDKWRVQIQGCVVAAALCAVITLIAMQWL MAFDAANLVMLYLLGVVVVALFYGRWPSVVATVINVVSFDLFFIAPRGTLAVSDVQYLLT FAVMLTVGLVIGNLTAGVRYQARVARYREQRTRHLYEMSKALAVGRSPQDIAATSEQFIA STFHARSQVLLPDDNGKLQPLTHPQGMTPWDDAIAQWSFDKGLPAGAGTDTLPGVPYQIL PLKSGEKTYGLVVVEPGNLRQLMIPEQQRLLETFTLLVANALERLTLTASEEQARMASER EQIRNALLAALSHDLRTPLTVLFGQAEILTLDLASEGS PHARQASEIRQHVLNTTRLVNN LLDMARIQSGGFNLKKEWLTLEEVVGSALQMLEPGLSSPINLSLPEPLTLIHVDGPLFER VLINLLENAVKYAGAQAEIGIDAHVEGENLQLDVWDNGPGLPPGQEQTIFDKFARGNKES AVPGVGLGLAI CRAIVDVHGGTITAFNRPEGGACFRVTLPQQTAPELEEFHEDM $>$ P 62517

MNKTTEYIDAMPIAASEKAALPKTDIRAVHQALDAEHRTWAREDDSPQGSVKARLEQAWP DSLADGQLI KDDEGRDQLKAMPEAKRSSMFPDPWRTNPVGRFWDRLRGRDVTPRYLARLT 
KEEQESEQKWRTVGTIRRYILLILTLAQTVVATWYMKTILPYQGWALINPMDMVGQDLWV SFMQLLPYMLQTGILILFAVLFCWVSAGFWTALMGFLQLLIGRDKYS ISASTVGDEPLNP EHRTALIMP I CNEDVNRVFAGLRATWESVKATGNAKHFDVYILSDSYNPDICVAEQKAWM ELIAEVGGEGQIFYRRRRRRVKRKSGNIDDFCRRWGSQYSYMVVLDADSVMTGDCLCGLV RLMEANPNAGI IQSSPKASGMDTLYARCQQFATRVYGPLFTAGLHFWQLGESHYWGHNAI IRVKPFIEHCALAPLPGEGSFAGS I LSHDFVEAALMRRAGWGVWIAYDLPGSYEELPPNL LDELKRDRRWCHGNLMNFRLFLVKGMHPVHRAVFLTGVMSYLSAPLWFMFLALSTALQVV HALTEPQYFLQPRQLFPVWPQWRPELAIALFASTMVLLFLPKLLS ILLIWCKGTKEYGGF WRVTLSLLLEVLFSVLLAPVRMLFHTVFVVSAFLGWEVVWNSPQRDDDSTSWGEAFKRHG SQLLLGLVWAVGMAWLDLRFLFWLAPIVFSLILSPFVSVISSRATVGLRTKRWKLFLIPE EYSPPQVLVDTDRFLEMNRQRSLDDGFMHAVFNPSFNALATAMATARHRASKVLEIARDR HVEQALNETPEKLNRDRRLVLLSDPVTMARLHFRVWNSPERYSSWVSYYEGIKLNPLALR KPDAASQ

$>$ P3 6646

MASKQLWRWHGITGDGNAQDGMLWAESRTLLLMALQQQMVTPLSLKRIAINSAQWRGDKS AEVIHQLATLLKAGLTLSEGLALLAEQHPSKQWQALLQSLAHDLEQGIAFSNALLPWSEV FPPLYQAMIRTGELTGKLDECCFELARQQKAQRQLTDKVKSALRYPI I ILAMA IMVVVAM LHFVLPEFAA IYKTFNTPLPALTQGIMTLADFSGEWSWLLVLFGFLLAIANKLLMRRPTW LIVRQKLLLR I P IMGSLMRGQKLTQIFT ILALTQSAGITFLQGVESVRETMRCPYWVQLL TQIQHDISNGQP IWLALKNTGEFSPLCLQLVRTGEASGSLDLMLDNLAHHHRENTMALAD NLAALLEPALL I ITGGI I GTLVVAMYLP I FHLGDAMSGMG

$>$ P 60778

MTNSNRIKLTWISFLSYALTGALVIVTGMVMGNIADYFNLPVSSMSNTFTFLNAGILISI FLNAWLMEIVPLKTQLRFGFLLMVLAVAGLMFSHSLALFSAAMF ILGVVSGITMS IGTFL VTQMYEGRQRGSRLLFTDSFFSMAGMIF PMIAAFLLARS IEWYWVYACIGLVYVAIF ILT FGCEFPALGKHAPKTDAPVEKEKWGIGVLFLSVAALCY ILGQLGF ISWVPEYAKGLGMSL NDAGTLVSNFWMSYMVGMWAFSF ILRFFDLQR ILTVLAGLAAILMYVFNTGTPAHMAWS I LALGFFSSAIYTTI ITLGSQQTKVPSPKLVNFVLTCGTIGTMLTFVVTGPIVEHSGPQAA LLTANGLYAVVFVMCFLLGFVSRHRQHNTLTSH

$>$ POADC6

MQPFGVLDRYIGKTIFTTIMMTLFMLVSLSGI I KFVDQLKKAGQGSYDALGAGMYTLLSV PKDVQIFFPMAALLGALLGLGMLAQRSELVVMQASGFTRMQVALSVMKTAI PLVLLTMAI GEWVAPQGEQMARNYRAQAMYGGSLLSTQQGLWAKDGNNFVYIERVKGDEELGGIS IYAF NENRRLQSVRYAATAKFDPEHKVWRLSQVDESDLTNPKQITGSQTVSGTWKTNLTPDKLG VVALDPDALS I SGLHNYVKYLKSSGQDAGRYQLNMWSKIFQPLSVAVMMLMALSFIFGPL RSVPMGVRVVTGISFGFVFYVLDQI FGPLTLVYGI PPI IGALLPSASFFLISLWLLMRKS $>$ P76425

MTEFTTLLQQGNAWFF I PSA I LLGALHGLEPGHSKTMMAAF I IAIKGT I KQAVMLGLAAT ISHTAVVWLIAFGGMVISKRFTAQSAEPWLQLISAVI I ISTAFWMFWRTWRGERNWLENM HGHDYEHHHHDHEHHHDHGHHHHHEHGEYQDAHARAHANDIKRRFDGREVTNWQILLFGL TGGL I PCPAA ITVLLICIQLKALTLGATLVVSFS IGLALTLVTVGVGAA I SVQQVAKRWS GFNTLAKRAPYFSSLLIGLVGVYMGVHGFMGIMR

$>Q 06067$

MHYMKWIYPRRLRNQMI LMAILMVIVPTLTIGYIVETEGRSAVLSEKEKKLSAVVNLLNQ ALGDRYDLYIDLPREERIRALNAELAP ITENITHAFPGIGAGYYNKMLDA I ITYAPSALY QNNVGVTIAADHPGREVMRTNTPLVYSGRQVRGDILNSMLPIERNGEILGYIWANELTED IRRQAWKMDVR I I IVLTAGLL I SLLL IVLFSRRLSANIDI I TDGLSTLAQN I PTRLPQLP GEMGQISQSVNNLAQALRETRTLNDLI IENAADGVIA IDRQGDVTTMNPAAEVITGYQRH ELVGQPYSMLFDNTQFYSPVLDTLEHGTEHVALEISFPGRDRTIELSVTTSR IHNTHGEM IGALV I F SDLTARKETQRRMAQAERLATLGELMAGVAHEVRNPLTA IRGYVQILRQQTSD PIHQEYLSVVLKEIDSINKVIQQLLEFSRPRHSQWQQVSLNALVEETLVLVQTAGVQARV DFISELDNELSP INADRELLKQVLLNILINAVQA I SARGKIRIQTWQYSDSQQAIS IEDN GCGIDLSLQKKIFDPFFTTKASGTGLGLALSQRI INAHQGDIRVASLPGYGATFTLILPI NPQGNQTV

$>$ POABI 8

MFGKLSLDAVPFHEPIVMVTIAGI ILGGLALVGLITYFGKWTYLWKEWLTSVDHKRLGIM YI IVAIVMLLRGFADAIMMRSQQALASAGEAGFLPPHHYDQIFTAHGVIMIFFVAMPFVI GLMNLVVPLQIGARDVAFPFLNNLSFWFTVVGVILVNVSLGVGEFAQTGWLAYPPLSGIE YSPGVGVDYWIWSLQLSGIGTTLTGINFFVTILKMRAPGMTMFKMPVFTWASLCANVLI I 
ASFPILTVTVALLTLDRYLGTHFFTNDMGGNMMMYINLIWAWGHPEVYILILPVFGVFSE IAATFSRKRLFGYTSLVWATVCITVLSFIVWLHHFFTMGAGANVNAFFGITTMI IAIPTG VKIFNWLFTMYQGRIVFHSAMLWTIGFIVTFSVGGMTGVLLAVPGADFVLHNSLFLIAHF HNVI I GGVVFGCFAGMTYWWPKAFGFKLNETWGKRAFWFWI IGFFVAFMPLYALGFMGMT RRLSQQIDPQFHTMLMIAASGAVLIALGILCLVIQMYVS IRDRDQNRDLTGDPWGGRTLE WATSSPPPFYNFAVVPHVHERDAFWEMKEKGEAYKKPDHYEEIHMPKNSGAGIVIAAFST IFGFAMIWHIWWLAIVGFAGMI ITWIVKSFDEDVDYYVPVAEIEKLENQHFDEITKAGLK NGN

$>Q 00185$

MKNRNNAVGPQIRAKKPKASKTVPILAGLSLGAGLQTATQYFAHSFQYQAGLGWNINHVY TPWS ILQWAGKWYGQYPDDFMRAASMGMVVSTVGLLGTAVTQMVKANTGKANDYLHGSAR WADKKDIQAAGLLPRPRTVVELVSGKHPPTSSGVYVGGWQDKDGKFHYLRHNGPEHVLTY APTRSGKGVGLVVPTLLSWAHSAVITDLKGELWALTAGWRKKHARNKVVRFEPASAQGSA CWNPLDEIRLGTEYEVGDVQNLATLIVDPDGKGLESHWQKTSQALLVGVILHALYKAKNE GTPATLPSVDGMLADPNRDVGELWMEMTTYGHVDGQNHPAVGSAARDMMDRPEEESGSVL STAKSYLALYRDPVVARNVSKSDFRI KQLMHHDDPVSLF IVTQPNDKARLRPLVRVMVNM IVRLLADKMDFENGRPVAHYKHRLLMMLDEFPSLGKLEILQESLAFVAGYGIKCYLICQD INQLKSRETGYGHDES ITSNCHVQNAYP PNRVETAEHLSKLTGTTTIVKEQITTSGRRTS ALLGNVSRTFQEVQRPLLTPDECLRMPGPKKSADGS IEEAGDMVVYVAGYPAIYGKQPLY FKDPIFQARAAVPAPKVSDKLIQTATVEEGEGITI

$>$ P60782

MSAIAPGMILIAYLCGS ISSAILVCRLCGLPDPRTSGSGNPGATNVLRIGGKGAAVAVLI FDVLKGMLPVWGAYELGVSPFWLGLIAIAACLGHIWPVFFGFKGGKGVATAFGAIAPIGW DLTGVMAGTWLLTVLLSGYSSLGAIVSALIAPFYVWWFKPQFTFPVSMLSCLILLRHHDN IQRLWRRQETKIWTKFKRKREKDPE

$>$ P17201

MGRLNRFRLGKDGRREQASLSRRGFLVTSLGAGVMFGFARPSSANQIFPLDRSLPGDGAF EPTIWCS IAPDGEITVNI I RAEMGQH IGTALARI IADEMEADWSKVR INYVDTDPKWGLM VTGGSWSVWMTWDVFRQAGAATRTAMVEEGARLLGTTPDKCTVASS IVSAGGKQISFGDI VAKGHPSHAFTPEEMAKLPLKPASERRLIGNAELKALDI PAKTNGTAIYGIDAKVEGMLY GRPKMPPTRYGSKVRSVDDTEAKKIKGYVRYLLIDDPSQVVQGWVVVLAESYSAAIRATD ALKVEWTPGETIHTSERDIQDRGRELINNKAGGVYIFNDDGVDQAFGSAHTVMDQEYTCA SVLHYQLEPTNALAFEKDGVYEIHAGNQWQSLILPTLAKSLQVPESKVILRSYLLGGGFG RRLNGDYMIPAALASKALGGKPVKLILTRSDDMQFDSFRSPSVQRVRMAFDASDRITAMD YQAAAGWPTGVMAEAFMEKGVDGKPYDQFAIAGGDHWYEVGAFRVRALRNDLAEKTFRPG WLRSVSPGWTSWGVECFLDEVAHRQKKDPAQFRLELLTGQGRNKGQAPDSVGGALRQAAV VRRLMEKVNWGKTSLPKDTAMGLATTAGQERGMPTWDRCVAQVHVDRSTGVVTCQKLTIL VDAGTVVDPDGAKAQTEGAALWGLSMVLFENTEIVNGMPVDRNLNTYTPLRIADTPEMDI EFLPSTEKPMGLGEPGTTVVGPAIGNAI FNAVGVRLRHMPVRPADVLRGLQNG

$>$ P18004

MNNPLEAVTQAVNSLVTALKLPDESAKANEVLGEMSFPQFSRLLPYRDYNQESGLFMNDT TMGFMLEA I P INGANES IVEALDHMLRTKLPRGI PLCIHLMSSQLVGDR IEYGLREFSWS GEQAERFNAITRAYYMKAAATQFPLPEGMNLPLTLRHYRVF ISYCSPSKKKSRADILEME NLVKI IRASLQGAS ITTQTVDAQAF IDIVGEM INHNPDSLYPKRRQLDPYSDLNYQCVED SFDLKVRADYLTLGLRENGRNSTARILNFHLARNPEIAFLWNMADNYSNLLNPELS ISCP FILTLTLVVEDQVKTHSEANLKYMDLEKKSKTSYAKWFPSVEKEAKEWGELRQRLGSGQS SVVSYFLNITAFCKDNNETALEVEQDILNSFRKNGFELISPRFNHMRNFLTCLPFMAGKG LFKQLKEAGVVQRAESFNVANLMPLVADNPLTPAGLLAPTYRNQLAF IDIFFRGMNNTNY NMAVCGTSGAGKTGLIQPLIRSVLDSGGFAVVFDMGDGYKSLCENMGGVYLDGETLRFNP FANITDIDQSAERVRDQLSVMASPNGNLDEVHEGLLLQAVRASWLAKENRARIDDVVDFL KNASDSEQYAESPTIRSRLDEMIVLLDQYTANGTYGQYFNSDEPSLRDDAKMVVLELGGL EDRPSLLVAVMFSLI IYIENRMYRTPRNLKKLNVIDEGWRLLDFKNHKVGEF IEKGYRTA RRHTGAYITITQNIVDFDSDKASSAARAAWGNSSYKI ILKQSAKEFAKYNQLYPDQFLPL QRDMIGKFGAAKDQWFSSFLLQVENHSSWHRLFVDPLSRAMYSSDGPDFEFVQQKRKEGL SIHEAVWQLAWKKSGPEMASLEAWLEEHEKYRSVA

$>$ P43009

MKKFDEINI I PFIDIMLVLLTVVLITASFISQGKIQVNVPKASTAVAFKSDELAKLLTVT ADKQLYFNDKP I SQEALEAEIAQWNKDQKVTLKIDAEASFQDFVTITDMLSKNEI KNVAI VSMKDKGKSAGKNSQESTPSQSVPTTP 
$>$ P31710

MKLKSGIVTGVALVLAYGLFLASYAPARLLTAVPLPAGMVVAEAAGTLWQGSLQRFSWRT LTLDDVHWNITFSDFMPALDIAFKNPEGIAGRGI IRGWQRAQFYQWQLSVPAGYLFSHMR FIVPIGAEGNVQLNLQEATVDRSGCQSLDANVTWPGARVKTPLGGLVLATPQATLRCQQG ALEANLRQTSSHLQLSGKGSVTPKGEYRFTGQLSSGNDLPATMKKLLATTGKANEQGART LNFQGRLL

$>$ POAEA5

MMFKQYLQVTKPGI I FGNLISVIGGFLLASKGS IDYPLFIYTLVGVSLVVASGCVFNNY I DRDIDRKMERTKNRVLVKGLISPAVSLVYATLLGIAGFMLLWFGANPLACWLGVMGFVVY VGVYSLYMKRHSVYGTLIGSLSGAAPPVIGYCAVTGEFDSGAAILLAIFSLWQMPHSYAI AIFRFKDYQAANI PVLPVVKGISVAKNHITLYI IAFAVATLMLSLGGYAGYKYLVVAAAV SVWWLGMALRGYKVADDRIWARKLFGFS I IAITALSVMMSVDFMVPDSHTLLAAVW $>$ P18006

MSSTQKPADVTAERRSHWWWTVPGCLAMVLLNAAVSYGIVRLNAPVTVAFNMKQTVDAFF DSASQKQLSEAQSKALSARFNTALEASLQAWQQKHHAVILVSPAVVQGAPDISREIQQDI ARRMRAEP

$>$ P77304

MSTANQKPTESVSLNAFKQPKAFYLIFSIELWERFGYYGLQGIMAVYLVKQLGMSEADS I TLFSSFSALVYGLVA IGGWLGDKVLGTKRVIMLGA IVLA IGYALVAWSGHDAGIVYMGMA AIAVGNGLFKANPSSLLSTCYEKNDPRLDGAFTMYYMSVNIGSFFSMIATPWLAAKYGWS VAFALSVVGLLITIVNFAFCQRWVKQYGSKPDFEP INYRNLLLTI IGVVALIAIATWLLH NQEVARMALGVVAFGIVVI FGKEAFAMKGAARRKMIVAFI LMLEA I I FFVLYSQMPTSLN FFA IRNVEHS I LGLAVEPEQYQALNPFWI I IGSP I LAA I YNKMGDTLPMPTKFA I GMVMC SGAFLILPLGAKFASDAGIVSVSWLVASYGLQS I GELMI SGLGLAMVAQLVPQRLMGF IM GSWFLTTAGANLIGGYVAGMMAVPDNVTDPLMSLEVYGRVFLQIGVATAVIAVLMLLTAP KLHRMTQDDAADKAAKAAVA

$>$ P69212

MYIYWILLGLAIATEITGTLSMKWASVSEGNGGF ILMLVMISLSYIFLSFAVKKIALGVA YALWEGIGILFITLFSVLLFDESLSLMKIAGLTTLVAGIVLIKSGTRKARKPELEVNHGA $\mathrm{V}$

$>$ P40608

MKLRTVAASLLLMLSATTVRASAADVGAPVPIYTEAELI KLIEQNKHLQRVRADNCQLVE DIVARATRINLPAYEFLYGDMLAWGVCVEQDVELGLYYMENAAQQGLPAALEQIGRYYSR GTLVQQDKERA I PYLREAASMGNLNAR I HLAELLLRDYGS PLDYEDAYRWLYNSVTADQR QHKRIAVLRRGLEQRMPQNIVARAKRRDMFW

$>$ P77307

MNSHNITNESLALALMLVVVAILISHKEKLALEKD ILWSVGRA I IQLI IVGYVLKY IFSV DDASLTLLMVLFICFNAAWNAQKRSKYIAKAF ISSFIAITVGAGITLAVLILSGS IEF IP MQVI P IAGMIAGNAMVAVGLCYNNLGQRVISEQQQIQEKLSLGATPKQASAILIRDSIRA ALI PTVDSAKTVGLVSLPGMMSGLIFAGIDPVKAIKYQIMVTFMLLSTASLSTI IACYLT YRKFYNSRHQLVVTQLKKK

$>$ P23482

MDALQLLTWSLILYLFASLASLFLLGLDRLAIKLSGITSLVGGVIGI ISGITQLHAGVTL VARFAPPFEFADLTLRMDSLSAFMVLVISLLVVVCSLYSLTYMREYEGKGAAAMGFFMNI FIASMVALLVMDNAFWF IVLFEMMSLSSWFLVIARQDKTS INAGMLYFFIAHAGSVLIMI AFLLMGRESGSLDFASFRTLSLSPGLASAVFLLAFFGFGAKAGMMPLHSWLPRAHPAAPS HASALMSGVMVKIGIFGILKVAMDLLAQTGLPLWWGI LVMA I GA I SALLGVLYALAEQD I KRLLAWSTVENVGI ILLAVGVAMVGLSLHDPLLTVVGLLGALFHLLNHALFKGLLFLGAG AI ISRLHTHDMEKMGALAKRMPWTAAACLIGCLA I SA I P PLNGF ISEWYTWQSLFSLSRV EAVALQLAGP IAMVMLAVTGGLAVMCFVKMYGITFCGAPRSTHAEEAQEVPNTMIVAMLL LAALCVLIALSASWLAPKIMHIAHAFTNTPPATVASGIALVPGTFHTQVTPSLLLLLLLA MPLLPGLYWLWCRSRRAAFRRTGDAWACGYGWENAMAPSGNGVMQPLRVVFSALFRLRQQ LDPTLRLNKGLAHVTARAQSTEPFWDERVIRPIVSATQRLAKEIQHLQSGDFRLYCLYVV AALVVLLIAIAV

$>$ P30845

MLKRLLKRPSLNLLAWLLLAAFYIS ICLNIAFFKQVLQALPLDSLHNVLVFLSMPVVAFS VINIVLTLSSFLWLNRPLACLF ILVGAAAQYF IMTYGIVIDRSMIANI IDTTPAESYALM TPQMLLTLGFSGVLAALIACWIKIKPATSRLRSVLFRGANILVSVLLILLVAALFYKDYA SLFRNNKELVKSLSPSNS IVASWSWYSHQRLANLPLVR I GEDAHRNPLMQNEKRKNLTIL 
IVGETSRAENFSLNGYPRETNPRLAKDNVVYFPNTASCGTATAVSVPCMFSDMPREHYKE ELAQHQEGVLDI IQRAGINVLWNDNDGGCKGACDRVPHQNVTALNLPDQCINGECYDEVL FHGLEEYINNLQGDGVIVLHTIGSHGPTYYNRYPPQFRKFTPTCDTNEIQTCTKEQLVNT YDNTLVYVDYIVDKAINLLKEHQDKFTTSLVYLSDHGESLGENGIYLHGLPYAIAPDSQK QVPMLLWLSEDYQKRYQVDQNCLQKQAQTQHYSQDNLFSTLLGLTGVETKYYQAADDILQ TCRRVSE

$>$ POAAM1

MQQKSDNVVSHYVFEAPVRIWHWLTVLCMAVLMVTGYFIGKPLPSVSGEATYLFYMGYIR LIHFSAGMVFTVVLLMR IYWAFVGNRYSRELF IVPVWRKSWWQGVWYEIRWYLFLAKRPS ADIGHNPIAQAAMFGYFLMSVFMI ITGFALYSEHSQYA I FAPFRYVVEFFYWTGGNSMD I HSWHRLGMWLIGAFVIGHVYMALREDIMSDDTVISTMVNGYRSHKFGKI SNKERS $>$ P0ABJ6 MSHSTDHSGASHGSVKTYMTGF ILS I ILTVI PFWMVMTGAASPAVILGTILAMAVVQVLV HLVCFLHMNTKSDEGWNMTAFVFTVLI IA I LVVGS IW IMWNLNYNMMMH

$>$ P76299

MSDESDDKTEAPTPHRLEKAREEGQI PRSRELTSLLILLVGVSVIWFGGVSLARRLSGML SAGLHFDHS I IND PNL I LGQ I I LL IREAMLALLPL I SGVVLVAL I SPVMLGGLVFSGKSL QPKFSKLNPLPGIKRMFSAQTGAELLKAILKTILVGSVTGFFLWHHWPQMMRLMAESPIT AMGNAMDLVGLCALLVVLGVI PMVGFDVFFQIFSHLKKLRMSRQDIRDEFKQSEGDPHVK GRIRQMQRAAARRRMMADVPKADVIVNNPTHYSVALQYDENKMSAPKVVAKGAGLVALRI REIGAENNVPTLEAPPLARALYRHAEIGQQI PGQLYAAVAEVLAWVWQLKRWRLAGGQRP VQPTHLPVPEALDFINEKPTHE

$>Q 46867$

MLKQKIKTIFEALLYIMLTYWLIDSFFAFNKYDWMLESGGNICS I PSVSGEDRILQAMIA AFFLLTPLI I LILRKLFMREMFEFWVYVFSLGICLVCGWWLFWGRF IFCY

$>$ POAEBO

MAEVTQLKRYDARP INWGKWFLIGIGMLVSAF ILLVPMIYIFVQAFSKGLMPVLQNLADP DMLHAIWLTVMIALIAVPVNLVFGILLAWLVTRFNFPGRQLLLTLLDIPFAVSPVVAGLV YLLFYGSNGPLGGWLDEHNLQIMFSWPGMVLVTI FVTCPFVVRELVPVMLSQGSQEDEAA ILLGASGWQMFRRVTLPNIRWALLYGVVLTNARA I GEFGAVSVVSGS IRGETLSLPLQIE LLEQDYNTVGSFTAAALLTLMA I ITLFLKSMLQWRLENQEKRAQQEEHHEH

$>$ P27843

MLRIFIPTSNGKISRRRYIFSFILINFIFAFLI IFFNDGEAGFLVIVSTIVLHYLVINMN CQRLRDSGFIYIKTYVFGTLAVYI ISI ITMIAEDFACSGNGSMI FL ICYFSTFSMLMLAP TDSSKQ

$>$ POAEB5

MIAELFTNNALNLVI I FGSCAALILMSFWFRRGNRKRKGFLFHAVQFLIYTI I ISAVGS I INYVIENYKLKFITPGVIDFICTSLIAVILTIKLFLLINQFEKQQI KKGRDITSARIMSR I IKITI IVVLVLLYGEHFGMSLSGLLTFGGIGGLAVGMAGKDILSNFFSGIMLYFDRPFS IGDWIRSPDRNIEGTVAEIGWRITKITTFDNRPLYVPNSLFSS ISVENPGRMTNRRITTT IGLRYEDAAKVGVIVEAVREMLKNHPAIDQRQTLLVYFNQFADSSLNIMVYCFTKTTVWA EWLAAQQDVYLKI IDIVQSHGADFAFPSQTLYMDNITPPEQGR

$>$ P77172

MKLNATYIKIRDKWWGLPLFLPSLILPIFAHINTFAHISSGEVFLFYLPLALMISMMMFF SWAALPGIALGI FVRKYAELGFYETLSLTANF I I I I I LCWGGYRVFTPRRNNVSHGDTRL ISQR I FWQIVFPATLFLILFQFAAFVGLLASRENLVGVMPFNLGTLINYQALLVGNLIGV PLCYFI IRVVRNPFYLRSYYSQLKQQVDAKVTKKEFALWLLALGALLLLLCMPLNEKSTI FSTNYTLSLLLPLMMWGAMRYGYKL I SLLWAVVLMIS I HSYQNY I P IYPGYTTQLTITSS SYLVFSFIVNYMAVLATRQRAVVRRIQRLAYVDPVVHLPNVRALNRALRDAPWSALCYLR IPGMEMLVKNYGIMLRIQYKQKLSHWLSPLLEPGEDVYQLSGNDLALRLNTESHQERITA LDSHLKQFRFFWDGMPMQPQIGVSYCYVRSPVNH IYLLLGELNTVAELS IVTNAPENMQR RGAMYLQRELKDKVAMMNRLQQALEHNHFFLMAQP ITGMRGDVYHEILLRMKGENDELIS PDSFLPVAHEFGLSSS IDMWVIEHTLQFMAENRAKMPAHRFAINLSPTSVCQARFPVEVS QLLAKYQIEAWQLIFEVTESNALTNVKQAQITLQHLQELGCQIAIDDFGTGYASYARLKN VNADLLKIDGSFIRNIVSNSLDYQIVAS I CHLARMKKMLVVAEYVENEEIREAVLSLGID YMQGYLIGKPQPLIDTLNEIEPIRESA

$>$ POABK2

MIDYEVLRFIWWLLVGVLLIGFAVTDGFDMGVGMLTRFLGRNDTERRIMINS IAPHWDGN QVWLITAGGALFAAWPMVYAAAF SGFYVAMILVLASLFFRPVGFDYRSKIEETRWRNMWD 
WGIFIGSFVPPLVIGVAFGNLLQGVPFNVDEYLRLYYTGNFFQLLNPFGLLAGVVSVGMI ITQGATYLQMRTVGELHLRTRATAQVAALVTLVCFALAGVWVMYGIDGYVVKSTMDHYAA SNPLNKEVVREAGAWLVNFNNTP ILWAI PALGVVLPLLTILTARMDKAAWAFVFSSLTLA CI ILTAGIAMFPFVMPSSTMMNASLTMWDATSSQLTLNVMTWVAVVLVPI ILLYTAWCYW KMFGRITKEDIERNTHSLY

$>$ P09129

MITQQI ISSELEVLKKHIDSGDIRI PSLWQGLKPGLI IMGWMI FCPLLMSFLITQKTSET LTAVLAGGWLGLI ILFIVARIRMLYFSLPEEFLKTSSVMRVISSKLKVYFIVYMGVIFLW SFLGGGI IYGFGAILVTVIMAFLIQLDIGRYQFVGVIDAINSYVKNKKLSRVK

$>$ P58593

MTQNLSQPPAVNAPESELDLVRYLDVLVANRWLIAGIAAVVMLLGATYAFLARPVYEADV LVQVEDNPNSAKSLLGDVSSLFDVKTDANAEIEILRSRMVVGKAVDNLHLYITAKPHYFP LIGAWVASRAKQLSEPGLFGLGGYTWGTELIDVDGFDVPEALEGQPFKLTALGNGRYRLE NKSLDTPIEGVVGEPLEAKQSVGTIQLLVNTLAAKAGAAFELQRDSRLKTLEMLQDKLKI SEKGKQSGI IGASLEGKNPALTAA IMNQIATEYVAQN I KRKAEEAERSLVFLDGLLPQLK LQLERAEMKYNEMRNLRGTFDLSEEGKAFLQESVTTETSLQELKQKRAELLTRFTASHPG VQAIDQQIAVMSGKVGAMTRRLKSLPNIEQDTVRLMRDVQVDNDLYVSLLNDMQQLKLVK AGKVGNVRLVDGAAVPEEPVKPKKLTVTALAGVLGVVLGVVAAFVRNTLFGGITEPQDIE EHTGLSVYATVPLSDVQIDLSSQLTTHKRGQYLLARRVPDDPS IESLRSLRTALQFAMQD SGNNLVVLTGPTPGVGKSFVSANLAAVIATGGKRVLLVDADMRKGYLHQYFGKDRKPGLL DLLAGDRS IEQVVHREVVPGLDF IATGLFPHNPSELLLNPRMVELMDTFRAQYDLVLIDT PPVLAVTDTAILAARAGTVLMVTRFERSTLGEIRETIKQLQHANVEVRGVVFNALDPNTY RYGYGSRYGRYRYVQYGYTSKPSAEAEAES

$>$ P3 7617

MSTPDNHGKKAPQFAAFKPLTTVQNANDCCCDGACSSTPTLSENVSGTRYSWKVSGMDCA ACARKVENAVRQLAGVNQVQVLFATEKLVVDADNDIRAQVESALQKAGYSLRDEQAAEEP QASRLKENLPLITLIVMMA I SWGLEQFNHPFGQLAF IATTLVGLYPIARQALRLIKSGSY FAIETLMSVAAIGALFIGATAEAAMVLLLFLIGERLEGWAASRARQGVSALMALKPETAT RLRKGEREEVAINSLRPGDVIEVAAGGRLPADGKLLSPFASFDESALTGESIPVERATGD KVPAGATSVDRLVTLEVLSEPGASAIDRILKLIEEAEERRAPIERFIDRFSRIYTPAIMA VALLVTLVPPLLFAASWQEWIYKGLTLLLIGCPCALVISTPAAITSGLAAAARRGALIKG GAALEQLGRVTQVAFDKTGTLTVGKPRVTAIHPATGISESELLTLAAAVEQGATHPLAQA IVREAQVAELA I PTAESQRALVGSGIEAQVNGERVLI CAAGKHPADAFTGLINELESAGQ TVVLVVRNDDVLGVIALQDTLRADAATAISELNALGVKGVILTGDNPRAAAAIAGELGLE FKAGLLPEDKVKAVTELNQHAPLAMVGDGINDAPAMKAAAIGIAMGSGTDVALETADAAL THNHLRGLVQMIELARATHANIRQNITIALGLKGIFLVTTLLGMTGLWLAVLADTGATVL VTANALRLLRRR

$>$ P37619

MNVFSQTQRYKALFWLSLFHLLVITSSNYLVQLPVS ILGFHTTWGAFSFPFIFLATDLTV RIFGAPLARR I IFAVMI PALLISYVISSLFYMGSWQGFGALAHFNLFVARIATASFMAYA LGQILDVHVFNRLRQSRRWWLAPTASTLFGNVSDTLAFFFIAFWRSPDAFMAEHWMEIAL VDYCFKVLISIVFFLPMYGVLLNMLLKRLADKSEINALQAS

$>$ P36672

MMSKINQTDIDRLIELVGGRGNIATVSHCITRLRFVLNQPANARPKEIEQLPMVKGCFTN AGQFQVVIGTNVGDYYQAL IASTGQAQVDKEQVKKAARHNMKWHEQLISHFAVIFFPLLP ALISGGLILGFRNV I GDLPMSNGQTLAQMYPSLQT IYDFLWLIGEA I FFYLPVGICWSAV KKMGGTPILGIVLGVTLVSPQLMNAYLLGQQLPEVWDFGMFS IAKVGYQAQVI PALLAGL ALGVIETRLKRIVPDYLYLVVVPVCSLILAVFLAHALIGPFGRMIGDGVAFAVRHLMTGS FAPIGAALFGFLYAPLVITGVHQTTLAIDLQMIQSMGGTPVWPLIALSNIAQGSAVIGI I ISSRKHNEREISVPAAISAWLGVTEPAMYGINLKYRFPMLCAMIGSGLAGLLCGLNGVMA NGIGVGGLPGILS IQP SYWQVFALAMAIA I I I P IVLTSF IYQRKYRLGTLDIV

$>$ POAECO

MEFLMDPS IWAGLLTLVVLEIVLGIDNLVFIAILADKLPPKQRDKARLLGLSLALIMRLG LLSLISWMVTLTKPLFTVMDFSFSGRDLIMLFGGIFLLFKATTELHERLENRDHDSGHGK GYASFWVVVTQIVILDAVFSLDAVITAVGMVNHLPVMMAAVVIAMAVMLLASKPLTRFVN QHPTVVVLCLSFLLMIGLSLVAEGFGFHI PKGYLYAAIGFS I I IEVFNQIARRNF IRHQS TLPLRARTADAILRLMGGKRQANVQHDADNPMPMP I PEGAFAEEERYMINGVLTLASRSL RGIMTPRGEISWVDANLGVDEIREQLLSSPHSLFPVCRGELDEI IGIVRAKELLVALEEG VDVAAIASASPA I IVPETLDP INLLGVLRRARGSFVIVTNEFGVVQGLVTPLDVLEAIAG 
EFPDADETPEI ITDGDGWLVKGGTDLHALQQALDVEHLADDDDIATVAGLVISANGHIPR VGDVIDVGPLHITIIEANDYRVDLVRIVKEQPAHDEDE

$>$ P08400

MLERLSWKRLVLELLLCCLPAFILGAFFGYLPWFLLASVTGLLIWHFWNLLRLSWWLWVD RSMTPPPGRGSWEPLLYGLHQMQLRNKKRRRELGNLIKRFRSGAESLPDAVVLTTEEGGI FWCNGLAQQILGLRWPEDNGQNILNLLRYPEFTQYLKTRDFSRPLNLVLNTGRHLEIRVM PYTHKQLLMVARDVTQMHQLEGARRNFFANVSHELRTPLTVLQGYLEMMNEQPLEGAVRE KALHTMREQTQRMEGLVKQLLTLSKIEAAPTHLLNEKVDVPMMLRVVEREAQTLSQKKQT FTFEIDNGLKVSGNEDQLRSAISNLVYNAVNHTPEGTHITVRWQRVPHGAEFSVEDNGPG IAPEHIPRLTERFYRVDKARSRQTGGSGLGLAIVKHAVNHHESRLNIESTVGKGTRFSFV IPERLIAKNSD

$>$ P31801

MSNAQEAVKTRHKETSLIFPVLALVVLFLWGSSQTLPVVIAINLLALIGILSSAFSVVRH ADVLAHRLGEPYGSLILSLSVVILEVSLISALMATGDAAPTLMRDTLYS I IMIVTGGLVG FSLLLGGRKFATQYMNLFGI KQYLIALFPLA I IVLVFPMALPAANF STGQALLVALISAA MYGVFLLIQTKTHQSLFVYEHEDDSDDDDPHHGKP SAHSSLWHAIWLI IHLIAVIAVTKM NASSLETLLDSMNAPVAFTGFLVALLILSPEGLGALKAVLNNQVQRAMNLFFGSVLATIS LTVPVVTLIAFMTGNELQFALGAPEMVVMVASLVLCHISFSTGRTNVLNGAAHLALFAAY LMTIFA

$>$ P0 8401

MRIGMRLLLGYFLLVAVAAWFVLAIFVKEVKPGVRRATEGTLIDTATLLAELARPDLLSG DPTHGQLAQAFNQLQHRPFRANIGGINKVRNEYHVYMTDAQGKVLFDSANKAVGQDYSRW NDVWLTLRGQYGARSTLQNPADPESSVMYVAAP IMDGSRLIGVLSVGKPNAAMAPVIKRS ERR ILWASAILLGIALVIGAGMVWWINRS IARLTRYADSVTDNKPVPLPDLGSSELRKLA QALESMRVKLEGKNYIEQYVYALTHELKSPLAAIRGAAEILREGPPPEVVARFTDNILTQ NARMQALVETLLRQARLENRQEVVLTAVDVAALFRRVSEARTVQLAEKKITLHVTPTEVN VAAEPALLEQALGNLLDNAIDFTPESGCITLSAEVDQEHVTLKVLDTGSGIPDYALSRIF ERFYSLPRANGQKSSGLGLAFVSEVARLFNGEVTLRNVQEGGVLASLRLHRHFT

$>$ P77328

MFNFAVSRESLLSGFQWFFFIFCNTVVVPPTLLSAFQLPQSSLLTLTQYAFLATALACFA QAFCGHRRAIMEGPGGLWWGTILTITLGEASRGTPINDIATSLAVGIALSGVLTMLIGFS GLGHRLARLFTPSVMVLFMLMLGAQLTTIFFKGMLGLPFGIADPNFKIQLPPFALSVAVM CLVLAMI I FLPQRFARYGLLVGTITGWLLWYFCFPSSHSLSGELHWQWFPLGSGGALSPG I ILTAVITGLVNI SNTYGAIRGTDVFYPQQGAGNTRYRRSFVATGFMTLITVPLAVIPFS PFVSSIGLLTQTGDYTRRSFIYGSVI CLLVALVPALTRLFCSI PLPVSSAVMLVSYLPLL FSALVFSQQITFTARNIYRLALPLFVGIFLMALPPVYLQDLPLTLRPLLSNGLLVGILLA VLMDNLIPWERIE

$>$ Q7A937

MDVIKKKHWWQSDALKWSVLGLLGLLVGYLVVLMYAQGEYLFAITTLILSSAGLYIFANR KAYAWRYVYPGMAGMGLFVLFPLVCTIAIAFTNYSSTNQLTFERAQEVLLDRSWQAGKTY NFGLYPAGDEWQLALSDGETGKNYLSDAFKFGREQKLQLKETTAQPEGERANLRVITQNR QALSDITAILPDGNKVMMSSLRQFSGTQPLYTLDGDGTLTNNQSGVKYRPNNQIGFYQS I TADGNWGDEKLSPGYTVTTGWKNFTRVFTDEGIQKPFLAIFVWTVVFSLITVFLTVAVGM VLACLVQWEALRGKAVYRVLLILPYAVPSFIS ILIFKGLFNQSFGEINMMLSALFGVKPA WFSDPTTARTMLI IVNTWLGYPYMMI LCMGLLKAI PDDLYEASAMDGAGPFQNFFKITLP LLIKPLTPLMIASFAFNFNNFVLIQLLTNGGPDRLGTTTPAGYTDLLVNYTYRIAFEGGG GQDFGLAAAIATLIFLLVGALAIVNLKATRMKFD

$>$ P04125

MAEVKQESLSGITEGEAKEFHKIFTSSILVFFGVAAFAHLLVWIWRPWVPGPNGYSALET LTQTLTYLS

$>$ P06739

MKTWLMGFSEFLLRYKLVWSETWKIRKQLDTPVREKDENEFLPAHLELIETPVSRRPRLV AYFIMGFLVIAFILSVLGQVEIVATANGKLTHSGRSKEIKPIENS IVKEI IVKEGESVRK GDVLLKLTALGAEADTLKTQSSLLQARLEQTRYQILSRS IELNKLPELKLPDEPYFQNVS EEEVLRLTSLIKEQFSTWQNQKYQKELNLDKKRAERLTVLARINRYENLSRVEKSRLDDF SSLLHKQAIAKHAVLEQENKYVEAVNELRVYKSQLEQIESEILSAKEEYQLVTQLFKNEI LDKLRQTTDNIGLLTLELAKNEERQQASVIRAPVSVKVQQLKVHTEGGVVTTAETLMVIV PEDDTLEVTALVQNKDIGF INVGQNAI I KVEAFPYTRYGYLVGKVKNINLDAIEDQRLGL VFNVI IS IEENCLSTGNKNI PLSSGMAVTAEIKTGMRSVISYLLSPLEESVTESLRER 
$>$ POABL8

MMFWRIFRLELRVAFRHSAEIANPLWFFLIVITLFPLS IGPEPQLLARIAPGI IWVAALL SSLLALERLFRDDLQDGSLEQLMLLPLPLPAVVLAKVMAHWMVTGLPLLILSPLVAMLLG MDVYGWQVMALTLLLGTPTLGFLGAPGVALTVGLKRGGVLLS ILVLPLTI PLLI FATAAM DAASMHLPVDGYLAILGALLAGTATLSPFATAAALRISIQ

$>$ P45570

MPTVITHAAVPLCIGLGLGSKVIPPRLLFAGI I LAMLPDADVLSFKFGVAYGNVFGHRGF THSLVFAFVVPLLCVF IGRRWFRAGLIRCWLFLTVSLLSHSLLDSVTTGGKGVGWLWPWS DERFFAPWQVIKVAPFALSRYTTPYGHQVI ISELMWVWLPGMLLMGMLWWRRR

$>$ POAFA2

MLKRCLSPLTLVNQVALIVLLSTAIGLAGMAVSGWLVQGVQGSAHAINKAGSLRMQSYRL LAAVPLSEKDKPLI KEMEQTAFSAELTRAAERDGQLAQLQGLQDYWRNELI PALMRAQNR ETVSADVSQFVAGLDQLVSGFDRTTEMRIETVVLVHRVMAVFMALLLVFTI IWLRARLLQ PWRQLLAMASAVSHRDFTQRANI SGRNEMAMLGTALNNMSAELAESYAVLEQRVQEKTAG LEHKNQI LSFLWQANRRLHSRAPLCERLSPVLNGLQNLTLLRDIELRVYDTDDEENHQEF TCQPDMTCDDKGCQLCPRGVLPVGDRGTTLKWRLADSHTQYGILLATLPQGRHLSHDQQQ LVDTLVEQLTATLALDRHQERQQQLIVMEERATIARELHDS IAQSLSCMKMQVSCLQMQG DALPESSRELLSQIRNELNASWAQLRELLTTFRLQLTEPGLRPALEASCEEYSAKFGFPV KLDYQLPPRLVPSHQA IHLLQIAREALSNALKHSQASEVVVTVAQNDNQVKLTVQDNGCG VPENAIRSNHYGMI IMRDRAQSLRGDCRVRRRESGGTEVVVTF I PEKTFTDVQGDTHE $>$ P77400 MMNTEGNNGNKPLGLWNVVS IGIGAMVGAGIFALLGQAALLMEASTWVAFAFGGIVAMFS GYAYARLGASYPSNGGI IDFFRRGLGNGVFSLALSLLYLLTLAVS IAMVARAFGAYAVQF LHEGSQEEHLI LLYALGI IAVMTLFNSLSNHAVGRLEVILVGI KMMI LLLLI IAGVWSLQ PAHISVSAPPSSGAFFSCIGITFLAYAGFGMMANAADKVKDPQVIMPRAFLVAIGVTTLL YISLALVLLSDVSALELEKYADTAVAQAASPLLGHVGYVIVVIGALLATASAINANLFAV FNIMDNMGSERELPKLMNKSLWRQSTWGNI IVVVLIMLMTAALNLGSLASVASATFLICY LAVFVVA IRLRHDIHASLP ILIVGTLVMLLVIVGF IYSLWSQGSRALIWI IGSLLLSLIV AMVMKRNKTV

$>$ POAFA5

MDWLLDVFATWLYGLKVIAITLAVIMFISGLDDFF IDVVYWVRRIKRKLSVYRRYPRMSY RELYKPDEKPLAIMVPAWNETGVIGNMAELAATTLDYENYHIFVGTYPNDPDTQRDVDEV CARFPNVHKVVCARPGPTSKADCLNNVLDAITQFERSANFAFAGF I LHDAEDVIS PMELR LFNYLVERKDLIQI PVYPFEREWTHFTSMTYIDEFSELHGKDVPVREALAGQVPSAGVGT CFSRRAVTALLADGDGIAFDVQSLTEDYDIGFRLKEKGMTEIFVRFPVVDEAKEREQRKF LQHARTSNMICVREYFPDTFSTAVRQKSRWI I GIVFQGFKTHKWTSSLTLNYFLWRDRKG AISNFVSFLAMLVMIQLLLLLAYESLWPDAWHFLS I FSGSAWLMTLLWLNFGLMVNRIVQ RVIFVTGYYGLTQGLLSVLRLFWGNLINFMANWRALKQVLQHGDPRRVAWDKTTHDFPSV TGDTRSLRPLGQILLENQVITEEQLDTALRNRVEGLRLGGSMLMQGLISAEQLAQALAEQ NGVAWES IDAWQI PSSLIAEMPASVALHYAVLPLRLENDELIVGSEDGIDPVSLAALTRK VGRKVRYVIVLRGQIVTGLRHWYARRRGHDPRAMLYNAVQHQWLTEQQAGEIWRQYVPHQ FLFAEILTTLGHINRSAINVLLLRHERSSLPLGKFLVTEGVISQETLDRVLTIQRELQVS MQSLLLKAGLNTEQVAQLESENEGE

$>$ P15701

MHIKRSIEKI PGGMMLVPLFLGALCHTFAPGAGKYFGSFTNGLISGTVPILAVWFFCMGA SIRLSATGTVLRKSGTLVVTKIAVAWVVAAVASRILPENGVEVGFFAGLSTLALVAAMDM TNGGLYAS IMQQYGTKEESGAFVLMSLESGPLMTMVILGTAGIASFEPHVFVGAVLPFLV GFALGNLDPELRDFFSRAVQTLI PFFAFALGNTIDLSVIGQTGLLGVLLGISVI I ITGIP LIVADKVLGGGDGTAGIAASSSAGAAVATPVLIAEMVPAFKPVAPAATTLVATSVIVTSV LVPI ITAMWSKRVKGGDGTVPKEDAVEEKAEQQRRRI IK

$>$ P76460

MIGRISRFMTRFVSRWLPDPLIFAMLLTLLTFVIALWLTPQTPISMVKMWGDGFWNLLAF GMQMALI IVTGHALASSAPVKSLLRTAASAAKTPVQGVMLVTFFGSVACVINWGFGLVVG AMFAREVARRVPGSDYPLLIACAYIGFLTWGGGF SGSMPLLAATPGNPVEHIAGLI PVGD TLFSGFNIFITVALIVVMPFITRMMMPKPSDVVS IDPKLLMEEADFQKQLPKDAPPSERL EESRILTLI IGALGIAYLAMYFSEHGFNITINTVNLMFMIAGLLLHKTPMAYMRAISAAA RSTAGILVQFPFYAGIQLMMEHSGLGGLITEFFINVANKDTFPVMTFFSSALINFAVPSG GGHWVIQGPFVIPAAQALGADLGKSVMAIAYGEQWMNMAQPFWALPALAIAGLGVRDIMG

YCITALLFSGVIFVIGLTLF 
$>$ P77338

MTMFQYYKRSRHFVFSAFIAFVFVLLCQNTAFARASSNGDLPTKADLQAQLDSLNKQKDL SAQDKLVQQDLTDTLATLDKIDR I KEETVQLRQKVAEAPEKMRQATAALTALSDVDNDEE TRKILSTLSLRQLETRVAQALDDLQNAQNDLASYNSQLVSLQTQPERVQNAMYNASQQLQ QIRSRLDGTDVGETALRPSQKVLMQAQQALLNAEIDQQRKSLEGNTVLQDTLQKQRDYVT ANSARLEHQLQLLQEAVNSKRLTLTEKTAQEAVSPDEAARIQANPLVKQELEINQQLSQR LITATENGNQLMQQNI KVKNWLERALQSERNI KEQIAVLKGSLLLSR ILYQQQQTLPSAD ELENMTNRIADLRLEQFEVNQQRDALFQSDAFVNKLEEGHTNEVNSEVHDALLQVVDMRR ELLDQLNKQLGNQLMMA INLQ INQQQLMSVSKNLKS I LTQQI FWVNSNRPMDWDWI KAFP QSLKDEFKSMKITVNWQKAWPAVF IAFLAGLPLLLIAGLIHWRLGWLKAYQQKLASAVGS LRNDSQLNTPKA I LIDLIRALPVCLI I LAVGLILLTMQLN I SELLWSFSKKLAI FWLVFG LCWKVLEKNGVAVRHFGMPEQQTSHWRRQIVRISLALLPIHFWSVVAELSPLHLMDDVLG QAMI FFNLLLIAFLVWPMCRESWRDKESHTMRLVTITVLS I I P IALMVLTATGYFYTTLR LAGRWIETVYLVI IWNLLYQTVLRGLSVAARRIAWRRALARRQNLVKEGAEGAEPPEEPT IALEQVNQQTLRITMLLMFALFGVMFWAIWSDLITVFSYLDS ITLWHYNGTEAGAAVVKN VTMGSLLFAI IASMVAWALI RNLPGLLEVLVLSRLNMRQGASYAITTILNYI I IAVGAMT VFGSLGVSWDKLQWLAAALSVGLGFGLQE I FGNFVSGLI I LFERPVRIGDTVTIGSFSGT VSKIRIRATTITDFDRKEVI I PNKAFVTERLINWSLTDTTTRLVIRLGVAYGSDLEKVRK VLLKAATEHPRVMHEPMPEVFFTAFGASTLDHELRLYVRELRDRSRTVDELNRTIDQLCR ENDINIAFNQLEVHLHNEKGDEVTEVKRDYKGDDPTPAVG

$>$ P76538

MKSTEFHPVHYDAHGRLRLPLLFWLVLLLQARTWVLFVIAGASREQGTALLNLFYPDHDN FWLGLIPGI PAVLAFLLSGRRATFPRTWRVLYFLLLLAQVVLLCWQPWLWLNGESVSGIG LALVVADIVALIWLLTNRRLRACFYEVKE

$>$ POA710

MKQFLDFLPLVVFFAFYKIYDIYAATAALIVATAIVLIYSWVRFRKVEKMALITFVLVVV FGGLTLFFHNDEFI KWKVTVIYALFAGALLVSQWVMKKPLIQRMLGKELTLPQPVWSKLN LAWAVFF I LCGLANIYIAFWLPQNIWVNFKVFGLTALTLIFTLLSGIYIYRHMPQEDKS $>$ P30878

MSISLTTKLSYGFGAFGKDFAIGIVYMYLMYYYTDVVGLSVGLVGTLFLVARIWDAINDP IMGWIVNATRSRWGKFKPWILIGTLTNSLVLFLLFSAHLFEGTAQVVFVCVTYILWGMTY TIMDI PFWSLVPTITLDKREREQLVPFPRFFASLAGFVTAGITLPFVSYVGGADRGFGFQ MFTLVLIAFFIASTIVTLRNVHEVYSSDNGVTAGRPHLTLKTIVGLIYKNDQLSCLLGMA LAYNIASNI INGFA IYYFTYVIGDADLFPYYLSYAGAANLLTLIVFPRLVKMLSRRILWA GASVMPVLSCAGLFAMALAD I HNAAL IVAAGIFLNIGTALFWVLQVIMVADTVDYGEFKL NIRCES IAYSVQTMVVKGGSAFAAFF IALVLGLIGYTPNVAQSAQTLQGMQF IMIVLPVL FFMMTLVLYFRYYRLNGDMLRKIQIHLLDKYRKTPPFVEQPDSPAISVVATSDVKA $>$ P39301

MHNI PGVWNTRLPLLQEIVMEILYNIFTVFFNQVMTNAPLLLGIVTCLGYILLRKSVSVI IKGTIKTI IGFMLLQAGSGILTSTFKPVVAKMSEVYGINGA ISDTYASMMATIDRMGDAY SWVGYAVLLALALN I CYVLLRR ITGIRT IMLTGH IMFQQAGLIAVTLF IFGYSMWTTI IC TAILVSLYWGITSNMMYKPTQEVTDGCGFS IGHQQQFASWIAYKVAPFLGKKEESVEDLK LPGWLNI FHDNIVSTAIVMTIFFGAILLSFGIDTVQAMAGKVHWTVYILQTGFSFAVAIF I ITQGVRMFVAELSEAFNGISQRLI PGAVLA IDCAA IYSFAPNAVVWGFMWGTIGQLIAV GILVACGSS IL I I PGF I PMFF SNAT I GVFANHFGGWRAALKICLVMGM IEI FGCVWAVKL TGMSAWMGMADWS I LAP PMMQGFFS I GIAFMAVI IVIALAYMFFAGRALRAEEDAEKQLA EQSA

$>Q 53174$

MDIAAAIGLIGAIVMVVGSMIYAGGVAPFVDI PSLVIVVAGTAF IVLAMKPLPVFLGHFK AMMKVFKPSRFDMNEVISTMVELSNLARKDGIMALEGKAVPDAFFEKGLQLLVDGTDEAK LVKQLKYEI KAMKARHEAYQGAVKAW ID I GPAMGMVGTLIGLVLMLGNMSDPKS I GPAMA VALLTTLYGALMANVIFAPILNKLEGYSADEVTYRELVIEGLRGIARGESARMIEDQMVC ALDRKQQMKR

$>$ POAAP7

MADFTLSKSLFSGKYRNASSTPGNIAYALFVLFCFWAGAQLLNLLVHAPGVYERLMQVQE TGRPRVEIGLGVGTIFGLI PFLVGCLIFAVVALWLHWRHR

$>$ P37630

MHISILAGIPEEFKVNIYIGWLFKLIPLIMGLICIALGGFVLESSGQSEYFVAGHVLISL AAICLALFTTAF I I ISQLTRGVNTFYNTLFP I IGYAGS I I TMIWGWALLAGNDVMADEFV 
AGHVIFGVGMIAACVSTVAASSGHFLLI PKNAAGSKSDGTPVQAYSSLIGNCLIAVPVLL TLLGF IWS ITLLRSADITPHYVAGHVLLGLTA I CACL IGLVATIVHQTRNTFSTKEHWLW CYWVIFLGSITVLQGIYVLVSSDASARLAPGI ILICLGMICYS I FSKVWLLALVWRRTCS LANRI PMI PVFTCLFCLFLASFLAEMAQTDMGYFI PSRVLVGLGAVCFTLFS IVS ILEAG SAKK

$>Q 51575$

MI PRRSSDITIKTRSDVLPFSGASSRWLQRYAPALLAVALI IAMS ISLAWQAAGWLRLQR SPVAVAASPVSHESIRSDPTRLARLFGTSAQDPNAPPPATNLDLVLKGSFVQSDPKLSSA I IQRQGDKPHRYAVGGEISDGVKLHAVYRDRVELQRGGRLESLPFPHRSGGLLASADDIT SENDS IEQLQSLQDENAAALRERLDALRQQMEATPIAEPAEEDSSEPTTTPTESD

$>$ P77416

MENLALTTLLLPFIGALVVSFSPQRRAAEWGVLFAALTTLCMLSLISAFYQADKVAVTLT LVNVGDVALFGLVIDRVSTLILFVVVFLGLLVTIYSTGYLTDKNREHPHNGTNRYYAFLL VFIGAMAGLVLSSTLLGQLLFFEITGGCSWALISYYQSDKAQRSALKALLITHIGSLGLY LAAATLFLQTGTFALSAMSELHGDARYLVYGGILFAAWGKSAQLPMQAWLPDAMEAPTP I SAYLHAASMVKVGVYI FARAI IDGGNI PHVIGGVGMVMALVTI LYGFLMYLPQQDMKRLL AWSTITQLGWMFFGLSLS I FGSRLALEGS IAY IVNHAFAKSLFFLVAGALSYSCGTRLLP RLRGVLHTLPLPGVGFCVAALAITGVPPFNGFFSKFPLFAAGFALSVEYWILLPAMILLM IESVASFAWF IRWFGRVVPGKPSEAVADAAPLPGSMRLVLIVLIVMSLISSVIAATWLQ $>$ P76470

MSTALLDAVVKKNRVRLIPFMLALYVLAFLDRSNIGFAKQTYQIDTGLSNEAYALGAGIF FVVYAFLGVPANLLMRKLGARTWIGTTTLLWGFLSAAMAWADTEAKFLIVRTLLRAAEAG FFPGMIYLTSQWFPQRNRAS IMGLFYMGAPLALTLGSPLSGALLEMHGFMGHPGWFWMFV IEGLLAVGAGVFTFFWLDDTPEQARFLSKQEKTLLINQLASEEQQKVTSRLSDALRNGRV WQLAI IYLTIQVAVYGLIFFLPTQVAALLGTKVGFTASVVTAI PWVAALFGTWLIPRYSD KTGERRNVAALTLLAAGIGIGLSGLLSPVMAIVALCVAAIGFIAVQPVFWTMPTQLLSGT ALAAGIGFVNLFGAVGGF IAP ILRVKAETLFASDAAGLLTLAAVAVIGSLI I FTLRVNRT VAQTDVAHH

$>$ P32482

MSSKNFSWRYSLAATVLLLSPFDLLASLGMDMYLPAVPFMPNALGTTASTIQLTLTTYLV MIGAGQLLFGPLSDRLGRRPVLLGGGLAYVVASMGLALTSSAEVFLGLRILQACGASACL VSTFATVRDIYAGREESNVIYGILGSMLAMVPAVGPLLGALVDMWLGWRAIFAFLGLGMI AASAAAWRFWPETRVQRVAGLQWSQLLLPVKCLNFWLYTLCYAAGMGSFFVFFSIAPGLM MGRQGVSQLGFSLLFATVAIAMVFTARFMGRVIPKWGSPSVLRMGMGCLIAGAVLLAITE IWALQSVLGF IAPMWLVGIGVATAVSVAPNGALRGFDHVAGTVTAVYFCLGGVLLGSIGT LI ISLLPRNTAWPVVVYCLTLATVVLGLSCVSRVKGSRGQGEHDVVALQSAGSTSNPNR $>$ P15643

MSKGI KMHNSVMRLT I PNKKI INYAPHIVTS I I LFF I CQQLAQLTWKI I LPVNFTDNALS SADMTSPAAPSAETALPRFTLFGLAEKTSASAPGGNLDQAPVSALRLRVTGLLASTDPSR AIA IMMKGNQQVSLGIGDNTPGGEAKI IA I SPDRLI VNYRGRNEA I PLFNDPPAVGKNSA APPARHLAQELRAQPQNILHYLNISPVMVNDKLSGYRLNPGKDPALFRQSGLRENDLAIA LNGLDLRDKEQARQVLAQLPELTEITLTVERDGQKNDIYLALRDE

$>$ P76473

MKSVRYLIGLFAFIACYYLLPISTRLLWQPDETRYAEISREMLASGDWIVPHLLGLRYFE KPIAGYWINS IGQWLFGANNFGVRAGVI FATLLTAALVTWFTLRLWRDKRLALLATVIYL SLFIVYAIGTYAVLDPF IAFWLVAGMCSFWLAMQAQTWKGKSAGFLLLGITCGMGVMTKG FLALAVPVLSVLPWVATQKRWKDLF IYGWLAVISCVLTVLPWGLAIAQREPNFWHYFFWV EHIQRFALDDAQHRAPFWYYVPVI IAGSLPWLGLLPGALYTGWKNRKHSATVYLLSWTIM PLLFFSVAKGKLPTYILSCFASLAMLMAHYALLAAKNNPLALRINGWINIAFGVTGI IAT FVVSPWGPMNTPVWQTFESYKVFCAWS IFSLWAFFGWYTLTNVEKTWPFAALCPLGLALL VGFS I PDRVMEGKHPQFFVEMTQESLQP SRYILTDSVGVAAGLAWSLQRDDI IMYRQTGE LKYGLNYPDAKGRFVSGDEFANWLNQHRQEGI ITLVLSVDRDEDINSLAI PPADAIDRQE RLVLIQYRPK

$>$ POABN1

ANNTTGFTRI I KAAGYSWKGLRAAWINEAAFRQEGVAVLLAVVIACWLDVDAITRVLLIS SVMLVMIVEILNSAIEAVVDRIGSEYHELSGRAKDMGSAAVLIAI IVAVITWCILLWSHF G

$>$ P75747

MPVLQWGMLCVLSLLLS IGFLAVHLPAALLLGPMIAGI IFSMRGITLQLPRSAFLAAQA I 
LGCMIAQNLTGS ILTTLAVNWP IVLAILLVTLLSSA IVGWLLVRYSSLPGNTGAWGSSPG GAAAMVAMAQDYGADIRLVAFMQYLRVLFVAGAAVLVTRMIMLGDNAEAVNQHIVWFPPVS INLLLTILLAVVAGTVGCLLRLPSGTMLI PMLAGAVLQSGQLITIELPEWLLAMAYMAIG WRIGLGFDKQILLRALRPLPQILLS IFALLAICAGMAWGLTRFMHIDFMTAYLATSPGGL DTVAVIAAGSNADMALIMAMQTLRLFS ILLTGPAIARF ISTYAPKRSA

$>$ P23597

MTGMDITTQDELNEAAMRDRASRDEERALRLGWWLVLAGFGGFLLWALLAPLDKGVAVQG NVVVSGNRKVIQHMQGGIVDRIQVKDGDRVAAGQVLLTLNAVDARTTSEGLGSQYDQLIA REARLLAEQRNQSSLAATPRLTQARQRPEMAAI IALQEDLLRSRQQSLKLEIDGVRAS ID GLETSLGALQKVMSSKQSEQATLSQQLQGLRPLAADNYVPRNKMLETERLFAQVSGELAQ TSGEVGRTRRDIQQQKLRIAQRQQEYDKEVNSELSDVQAKLNEVISQREKADFNLANVQV RAPVAGTVVDMKI FTEGGVIAPGQVMMDIVPEDQPLLVDGRI PVEMVDKVWSGLPVELQF TAFSQSTTPRVPGTVTLLSADRLVDEKDGTPYYGLRIQVSEEGKRSLHGLEI KPGMPVQG FVRTGERSFINYLFKPLMDRMHLALTEE

$>$ P45800

MSTIVIFLAALLACSLLAGWLIKVRSRRRQLPWTNAFADAQTRKLTPEERSAVENYLESL TQVLQVPGPTGASAAP ISLALNAESNNVMMLTHAITRYGISTDDPNKWRYYLDSVEVHLP PFWEQYINDENTVELIHTDSLPLVISLNGHTLQEYMQETRSYALQPVPSTQAS IRGEESE QIELLNIRKETHEEYALSRPRGLREALLIVASFLMFFFCLITPDVFVPWLAGGALLLLGA GLWGLFAPPAKSSLREIHCLRGTPRRWGLFGENDQEQINNISLGI IDLVYPAHWQPYIAQ DLGQQTDIDIYLDRHVVRQGRYLSLHDEVKNFPLQHWLRSTI IAAGSLLVLFMLLFWI PL DMPLKFTLSWMKGAQTIEATSVKQLADAGVRVGDTLRISGTGMCNIRTSGTWSAKTNSPF LPFDCSQI IWNDARSLPLPESELVNKATALTEAVNRQLHPKPEDESRVSASLRSAIQKSG MVLLDDFGDIVLKTADLCSAKDDCVRLKNALVNLGNSKDWDALVKRANAGKLDGVNVLLR PVSAESLDNLVATSTAPFITHETARAAQSLNSPAPGGFLIVSDEGSDFVDQPWPSASLYD YPPQEQWNAFQKLAQMLMHTPFNAEGIVTKIFTDANGTQHIGLHPI PDRSGLWRYLSTTL LLLTMLGSAIYNGVQAWRRYQRHRTRMMEIQAYYESCLNPQLITPSESLIE

$>$ P37642

MTQENEIKRPIQDLEHEPIKPLDNSEKGSKVSQALETVTTTAEKVQRQPVIAHLIRATER FNDRLGNQFGAAITYFSFLSMI P ILMVSFAAGGFVLASHPMLLQDIFDKILQNISDPTLA ATLKNTINTAVQQRTTVGLVGLAVALYSGINWMGNLREAIRAQSRDVWERSPQDQEKFWV KYLRDFISLIGLLIALIVTLS ITSVAGSAQQMI ISALHLNS IEWLKPTWRLIGLA IS I FA NYLLFFWIFWRLPRHRPRKKALIRGTFLAAIGFEVIKIVMTYTLPSLMKSPSGAAFGSVL GLMAFFYFFARLTLFCAAWIATAEYKDDPRMPGKTQP

$>Q 06401$

MAEQEYDLIVVGSGAGACWAPIRAQEQGLKTLVVEKTELFGGTSALSGGGIWI PLNYDQK TAGIKDDLETAFGYMKRCVRGMATDDRVLAYVETASKMAEYLRQIGI PYRAMAKYADYYP HIEGSRPGGRTMDPVDFNAARLRVTALETMRPGP PGNQLFGRMS ISAFEAHSMLSRELKS RFTILGIMLKYFLDYPWRNKTRRDRRMTGGQALVAGLLTAANKARVEMWCNSPLKELVQD ASGRVTGVIVERNGQRQQINARRGVLLGAGGFERNQEMRDQYLNKPTRLVDGNPCGRQYG DAHRAGQAWAHTGADGLVLGRAHHGCSQGAGLSRHFRGTLAAGVHGGQRQGAALPQRVRP VSGI PAAMLAENAKGNGGVPAWIVFDASFRAQNPMGPLMPGSAVPDSKVRKSWLNNVYWK GRRWKIWRADRRGRAGLQVSARRMTEYARAGKDLDFDRGGNVFDRYYGDPRLKNPNLGP I EKGPFYAMRLWPGEIGTKGGLLTDREGRVLDTQGR I IEGLYCVGNNSASVMAPAYAGAGS TLGPAMTFAFRAVADMVGKPLPLENPHLLGKTV

$>$ P19934

MSKATEQNDKLKRAI I ISAVLHVILFAALIWSSFDENIEASAGGGGGSS IDAVMVDSGAV VEQYKRMQSQESSAKRSDEQRKMKEQQAAEELREKQAAEQERLKQLEKERLAAQEQKKQA EEAAKQAELKQKQAEEAAAKAAADAKAKAEADAKAAEEAAKKAAADAKKKAEAEAAKAAA EAQKKAEAAAAALKKKAEAAEAAAAEARKKAATEAAEKAKAEAEKKAAAEKAAADKKAAA EKAAADKKAAEKAAAEKAAADKKAAAEKAAADKKAAAAKAAAEKAAAAKAAAEADD I FGE LSSGKNAPKTGGGAKGNNASPAGSGNTKNNGASGADINNYAGQI KSAIESKFYDASSYAG KTCTLRI KLAPDGMLLDI KPEGGDPALCQAALAAAKLAKI PKP PSQAVYEVFKNAPLDFK $\mathrm{P}$

$>$ P29486

MIKKI ISVFLLLACI ITLAFTAFFYHSKLSDQTKS ISSLSSQQAQERLQSYQDSLDFYKK LNTSLSVAIANSLRDKAVEELNAIALRIQENHGFIGVTFASLDGTMFTDIGTLDWNAKTL RRDWFVKTVELGTKHYTAFDIDKTTGQHVLTIATPVYVGNDIVGSVALDIAGDQIASPNG SGMFMMTDRNFNVFASDLTHSTLIGKDLTKEKPLFKNLVSGQYVTFSDADSHWFAVSQTE 
IDGENKLFTI IDIQQIVQTYKRDIQLI IAGFSGF SCVMLIGLYWVLSKELSGVRQIREWI LALSDGQIKERRPIKFHNELDTIAQSLENLQFRLLDVVRNSHRTMNDLS I KQTDITYS IE GNTNNSQQELGLIEQVATATTQLSCTSFDVMQQAQSAELNAETAQKLIAESHDI IDSSSK QTEMVTLS IHESQQI INQLREFSDNISSVTDVINNISDQTNLLALNAAIEAARAGEQGRG FAVVADEVRSLAVKTQQSTIDIQGI I LKLQEQSQLADQVMTRNVSLI HETQVANRALIAS FNLISDKVLEISNINS IVSTAANEQKIVTEDVAKQMEDIRYLVQENLSAMERTKQANQNI SDLTTNLNDALSFFKIELTS

$>$ POAFC3

MSMSTSTEVIAHHWAFAIFLIVAIGLCCLMLVGGWFLGGRARARSKNVPFESGIDSVGSA RLRLSAKFYLVAMFFVI FDVEALYLFAWSTS IRESGWVGFVEAAIF I FVLLAGLVYLVRI GALDWTPARSRRERMNPETNSIANRQR

$>$ P33361

MTYFRINPVLALLLLLTAIAAALPFISYAPNRLVSGEGRHLWQLWPQTIWMLVGVGCAWL TACF I PGKKGS I CAL I LAQFVFVLLVWGAGKAATQLAQNGSALARTSLGSGFWLAAALAL LACSDAIRR I STHPLWRWLLHMQIA I I PLWLLYSGTLNDLSLMKEYANRQDVFDDALAQH LTLLFGAVLPALVIGVPLGIWCYFSTARQGAIFSLLNVIQTVPSVALFGLLIAPLAALVT AFPWLGTLGIAGTGMTPALIALVLYALLPLVRGVVVGLNQI PRDVLESARAMGMSGAQRF LHVQLPLALPVFLRSLRVVMVQTVGMAVIAALIGAGGFGALVFQGLLSSAIDLVLLGVIP VIVLAVLTDALFDLLIALLKVKRND

$>Q 05605$

MTRTQPSASPTPSRAWRAIAALMFSLVLAPVAMADEPTANASTPAAAAAPATPAAAPAPA ADGSAPVADAPAAAPVDAPVAVDPGVEALVEDTTLGMAHDLSPWGMYKNADIVVKIVMIG LAIAS I ITWTIWIAKGFELMGAKRRLRGEIAQLKKSASLKEASEVSNKEGTLAHTLVHDA LEEMRLSANTREKEGI KERVAFRLERLVAASGRNMSSGTGVLATIGSTAPFVGLFGTVWG IMNSFIGIAKTQTTNLAVVAPGIAEALLATALGLVAAI PAVVIYNVFARS IAGYKAQVSD ASAQVLLLVSRDLDHQGSERAAPHMVKVG

$>$ P77354

MSSERDLVNFLGDFSMDVAKAVIAGGVATAIGSLASFACVSFGFPVILVGGAILLTGIVC TVVLNEIDAQCHLSEKLKYAIRDGLKRQQELDKWKRENMTPFMYVLNTPPVI

$>$ P08369

MLKSPLFWKMTSLFGAVLLLLIPIMLIRQVIVERADYRSDVEDAIRQSTSGPQKLVGPLI AI PVTELYTVQEEDKTVERKRSF IHFWLPESLMVDGNQNVEERKIGIYTGQVWHSDLTLK ADFDVSRLSELNAPNITLGKPFIVISVGDARGIGVVKAPEVNGTALTIEPGTGLEQGGQG VHI PLPEGDWRKQNLKLNMALNLSGTGDLSVVPGGRNSEMTLTSNWPHPSFLGDFLPAKR EVSESGFQAHWQSSWFANNLGERFASGNDTGWENFPAFSVAVTTPADQYQLTDRATKYAI LLIALTFMAFFVFETLTAQRLHPMQYLLVGLSLVMFYLLLLALSEHTGFTVAWI IASLIG AIMNGIYLQAVLKGWCNSMLFTLALLLLDGVMWGLLNSADSALLLGTSVLVVALAGMMFV TRNIDWYAFSLPKMKASKEVTTDDELRIWK

$>$ P75685

MEKYLHLLSRGDKIGLTLIRLSIAIVFMWIGLLKFVPYEADSITPFVANSPLMSFFYEHP EDYKQYLTHEGEYKPEARAWQTANNTYGFSNGLGVVEVI IALLVLANPVNRWLGLLGGLM AFTTPLVTLSFLITTPEAWVPALGDAHHGFPYLSGAGRLVLKDTLMLAGAVMIMADSARE ILKQRSNESSSTLKTEY

$>$ POAB12

MRTVLNILNFVLGGFATTLGWLLATLVS IVLIFTLPLTRSCWEITKLSLVPYGNEAIHVD ELNPAGKNVLLNTGGTVLNI FWL I FFGWWLCLMHIATGIAQCIS I I GI PVGIANFKIAA I ALWPVGRRVVSVETAQAAREANARRRFE

$>$ P54 702

MIQVTSEQWLYWLHLYFWPLLRVLALISTAP ILSERAI PKRVKLGLGIMITLVIAPSLPA NDTPLFS IAALWLAMQQILIGIALGFTMQFAFAAVRTAGEF IGLQMGLSFATFVDPGSHL NMPVLAR IMDMLAMLLFLTFNGHLWLISLLVDTFHTLP IGSNPVNSNAFMALARAGGLIF LNGLMLALPV ITLLLTLNLALGLLNRMAPQLS IFVIGFPLTLTVGIMLMAALMPLIAPFC EHLFSEIFNLLADIVSEMPINNNP

$>$ POAA4 7

MSHNVTPNTSRVELRKTLTLVPVVMMGLAYMQPMTLFDTFGIVSGLTDGHVPTAYAFALI AILFTALSYGKLVRRYPSAGSAYTYAQKS ISPTVGFMVGWSSLLDYLFAPMINILLAKIY FEALVPS I PSWMFVVALVAFMTAFNLRSLKSVANFNTVIVVLQVVLIAVILGMVVYGVFE GEGAGTLASTRPFWSGDAHVI PMITGATILCFSFTGFDGISNLSEETKDAERVIPRAIFL TALIGGMIFIFATYFLQLYFPDISRFKDPDASQPEIMLYVAGKAFQVGALIFSTITVLAS 
GMAAHAGVARLMYVMGRDGVFPKSFFGYVHPKWRTPAMNI ILVGAIALLAINFDLVMATA LINFGALVAFTFVNLSVISQFWIREKRNKTLKDHFOYLFLPMCGALTVGALWVNLEESSM VLGLIWAAIGLIYLACVTKSFRNPVPQYED

$>$ P18200

MTILPRHKDVAKSRLKMSNPWHLLAVGFGSGLSPIVPGTMGSLAAIPFWYLMTFLPWQLY SLVVMLGICIGVYLCHQTAKDMGVHDHGS IVWDEF I GMWITLMALPTNDWQWVAAGFVIF RILDMWKPWP IRWFDRNVHGGMGIMIDDIVAGVISAGILYFIGHHWPLGILS

$>$ POAFD4

MSWISPELIEILLTILKAVVILLVVVTCGAFMSFGERRLLGLFQNRYGPNRVGWGGSLQL VADMI KMFFKEDWI PKFSDRVI FTLAPMIAFTSLLLAFAIVPVSPGWVVADLNIGILFFL MMAGLAVYAVLFAGWSSNNKYSLLGAMRASAQTLSYEVFLGLSLMGVVAQAGSFNMTDIV NSQAHVWNVI PQFFGFITFAIAGVAVCHRHPFDQPEAEQELADGYHIEYSGMKFGLFFVG EYIGIVTISALMVTLFFGGWQGPLLPPF IWFALKTAFFMMMF I LIRASLPRPRYDQVMSF GWKICLPLTLINLLVTAAVILWQAQ

$>$ P75830

MKKRKTVKKRYVIALVIVIAGLITLWRILNAPVPTYQTLIVRPGDLQQSVLATGKLDALR KVDVGAQVSGQLKTLSVAIGDKVKKDQLLGVIDPEQAENQI KEVEATLMELRAQRQQAEA ELKLARVTYSRQQRLAQTKAVSQQDLDTAATEMAVKQAQIGTIDAQI KRNQASLDTAKTN LDYTRIVAPMAGEVTQITTLQGQTVIAAQQAPNILTLADMSAMLVKAQVSEADVIHLKPG QKAWFTVLGDPLTRYEGQIKDVLPTPEKVNDAIFYYARFEVPNPNGLLRLDMTAQVHIQL TDVKNVLTIPLSALGDPVGDNRYKVKLLRNGETREREVTIGARNDTDVEIVKGLEAGDEV VIGEAKPGAAQ

$>$ P21210

MNPHDLEWLNRIGERKDIMLAVLLLAVVFMMVLPLPPLVLDILIAVNMTISVVLLMIAIY INSPLQFSAFPAVLLVTTLFRLALSVSTTRMILLQADAGQIVYTFGNFVVGGNFIVGIVI FLIITIVQFLVITKGSERVAEVSARFSLDAMPGKQMS IDGDMRAGVIDVNEARERRATIE KESQMFGSMDGAMKFVKGDA IAGLI I IFVNILGGVTIGVTQKGLAAAEALQLYS ILTVGD GMVSQVPALLIAITAGI IVTRVSSEDSSDLGSDIGKQVVAQPKAMLIGGVLLLLFGLI PG FPTVTFLILALLVGCGGYMLSRKQSRNDEANQDLQSLLTSGSGAPAARTKAKTSGANKGR LGEQEAFAMTVPLLIDVDSSQQEALEAIALNDELVRVRRALYLDLGVPFPGIHLRFNEGM GEGEYLISLQEVPVARGELKAGYLLVRESVSQLELLGI PYEKGEHLLPDQETFWVSVEYE ERLEKSQLEFFSHSQVLTWHLSHVLREYAEDFIGIQETRYLLEQMEGGYGELI KEVQRIV PLQRMTEILQRLVGEDISIRNMRS I LEAMVEWGQKEKDVVQLTEYIRSSLKRYICYKYAN GNNILPAYLFDQEVEEKIRSGVRQTSAGSYLALDPAVTESLLEQVRKTIGDLSQIQSKPV LIVSMDIRRYVRKLIESEYYGLPVLSYQELTQQINIQPLG

$>$ POADJ8

MDNKISTYSPAFS IVSWIALVGGIVTYLLGLWNAEMQLNEKGYYFAVLVLGLFSAASYQK TVRDKYEGI PTTS I YYMTCLTVF I ISVALLMVGLWNATLLLSEKGFYGLAFFLSLFGAVA VQKNIRDAGINPPKETQVTQEEYSE

$>$ POABP3

MLTFIELLIGVVVIVGVARYI IKGYSATGVLFVGGLLLLI ISAIMGHKVLPSSQASTGYS ATDIVEYVKILLMSRGGDLGMMIMMLCGFAAYMTHIGANDMVVKLASKPLQY INSPYLLM IAAYFVACLMSLAVSSATGLGVLLMATLFPVMVNVGISRGAAAA I CASPAAI I LAPTSGD VVLAAQASEMSLIDFAFKTTLP I S IAAI I GMA IAHF FWQRYLDKKEH I SHEMLDVSEITT TAPAFYAILPFTPI IGVLI FDGKWGPQLHI ITILVICMLIAS ILEFLRSFNTQKVFSGLE VAYRGMADAFANVVMLLVAAGVFAQGLST IGF IQSLIS IATSFGSAS I I LMLVLVILTML AAVTTGSGNAPFYAFVEMI PKLAHSSGINPAYLTI PMLQASNLGRTLSPVSGVVVAVAGM AKISPFEVVKRTSVPVLVGLVIVIVATELMVPGTAAAVTGK

$>$ P39401

MSELLSFALFLASVLIYAWKAGRNTWWFAATLTVLGLFVVLNITLFASDYFTGDGINDAV LYTLTNSLTGAGVSKYILPGIGIVLGLTAVFGALGWILRRRRHHPHHFGYSLLALLLALG SVDASPAFRQITELVKSQSRDGDPDFAAYYKEPSKTI PDPKLNLVYIYGESLERTYFDNE AFPDLTPELGALKNEGLDFSHTQQLPGTDYTIAGMVASQCGIPLFAPFEGNASASVSSFF PQNICLGDILKNSGYQNYFVQGANLRFAGKDVFLKSHGFDHLYGSEELKSVVADPHYRND WGFYDDTVLDEAWKKFEELSRSGQRFSLFTLTVDTHHPDGF ISRTCNRKKYDFDGKPNQS FSAVSCSQENIATFINKIKASPWFKDTVIVVSSDHLAMNNTAWKYLNKQDRNNLFFVIRG DKPQQETLAVKRNTMDNGATVLDI LGGDNYLGLGRSSLSGQSMSEIFLNI KEKTLAWKPD I IRLWKFPKEMKEFTIDQQKNMIAFSGSHFRLPLLLRVSDKRVEPLPESEYSAPLRFQLA DFAPRDNFVWVDRCYKMAQLWAPELALSTDWCVSQGQLGGQQIVQHVDKTTWQGKTAFKD 
TVIDMARYKGNVDTLKIVDNDIRYKADSFIFNVAGAPEEVKQFSGISRPESWGRWSNAQL GDEVKIEYKHPLPKKFDLVITAKAYGNNASRPI PVRVGNEEQTLVLGNEVTTTTLHFDNP TDADTLVIVPPEPVSTNEGNILGHSPRKLGIGMVEIKVVEREG

$>$ POAAS3

MELMVVHPHIFWLSLGGLLLAAEMLGGNGYLLWSGVAAVITGLVVWLVPLGWEWQGVMFA ILTLLAAWLWWKWLSRRVREQKHSDSHLNQRGQQLIGRRFVLESPLVNGRGHMRVGDSSW PVSASEDLGAGTHVEVIAIEGITLHIRAVS

$>$ P39338

MAQVINEMDVPSHSFVFHGTGERYFLICVVNVLLTI ITLGIYLPWALMKCKRYLYANMEV NGQRFSYGITGGNVFVSCLFFVFFYFAILMTVSADMPLVGCVLTLLLLVLLIFMAAKGLR HQALMTSLNGVRFSFNCSMKGFWWVTFFLPILMAIGMGTVFFISTKMLPANSSSSVI ISM VLMAIVGIVSIGIFNGTLYSLVMSFLWSNTSFGIHRFKVKLDTTYCIKYAILAFLALLPF LAVAGYI I FDQ I LNAYDSSVYANDD IENLQQFMEMQRKMI IAQLIYYFGIAVSTSYLTVS LRNHFMSNLSLNDGR IRFRLTLTYHGMLYRMCALVVISGITGGLAYPLLKIWMIDWQAKN TYLLGDLDDLPLINKEEQPDKGFLASISRGVMPSLPFL

$>$ P3 7661

MRYIKSITQQKLSFLLAIYIGLFMNGAVFYRRFGSYAHDFTVWKGISAVVELAATVLVTF FLLRLLSLFGRRSWRILASLVVLFSAGASYYMTFLNVVIGYGI IASVMTTDIDLSKEVVG LNFILWLIAVSALPLILIWNNRCRYTLLRQLRTPGQR IRSLAVVVLAGIMVWAPIRLLDI QQKKVERATGVDLPSYGGVVANSYLPSNWLSALGLYAWARVDESSDNNSLLNPAKKFTYQ APQNVDDTYVVF I IGETTRWDHMGIFGYERNTTPKLAQEKNLAAFRGYSCDTATKLSLRC MFVRQGGAEDNPQRTLKEQNIFAVLKQLGFSSDLYAMQSEMWFYSNTMADNIAYREQIGA EPRNRGKPVDDMLLVDEMQQSLGRNPDGKHLI ILHTKGSHFNYTQRYPRSFAQWKPECIG VDSGCTKAQMINSYDNSVTYVDHFISSVIDQVRDKKAIVFYAADHGES INEREHLHGTPR ELAP PEQFRVPMMVWMSDKYLENPANAQAFAQLKKEADMKVPRRHVELYDTIMGCLGYTS PDGGINENNNWCHI PQAKEAAAN

$>$ P38392

MLDVFTPLLKLFANEPLERLMYTI I IFGLTLWLI PKEFTVAFNAYTEIPWLFQI IVFAFS FVVAISFSRLRAHIQKHYSLLPEQRVLLRLSEKEIAVFKDFLKTGNLI ITSPCRNPVMKK LERKGIIQHQSDSANCSYYLVTEKYSHFMKLFWNSRSRRFNR

$>$ P3 9196

MSESVHTNTSLWSKGMKAVIVAQFLSAFGDNALLFATLALLKAQFYPEWSQP I LQMVFVG AYILFAPFVGQVADSFAKGRVMMFANGLKLLGAAS ICFGINPFLGYTLVGVGAAAYSPAK YGILGELTTGSKLVKANGLMEASTIAAI LLGSVAGGVLADWHVLVALAACALAYGGAVVA NIYI PKLAAARPGQSWNLINMTRSFLNACTSLWRNGETRFSLVGTSLFWGAGVTLRFLLV LWVPVALGITDNATPTYLNAMVAIGIVVGAGAAAKLVTLETVSRCMPAGILIGVVVLIFS LQHELLPAYALLMLIGVMGGFFVVPLNALLQERGKKSVGAGNAIAVQNLGENSAMLLMLG IYSLAVMIGIPVVPIGIGFGALFALAITALWIWQRRH

$>$ P37739

MRDTTGGPAGAEVWTVPGLLGARKLDLLALI PLVAIVALMTLVGALLFAVAQSDANRARA KLATDALWVEQTLRFQMAVDEDVLVRLALDASAGASQQALSARARLHLAANPETLGLRWY DATGRLIAAVPEGPGPAEAALVRQLLASGALPPRPVYGPVRDGRVVLAERVSASGGVVVA TVSLPMMLERHLPWWIAEQYGVRISDTSGVLAERARRPIAAAAPRHGISFDPPLAGTTLE IMAYDAPDAFGNAALLAAIGALSVFAVLAMVVLHRNALRRRMAEDRLRAEMAFRRAMEES LTVGMRAKDLSGR I LYVNGAFCKLVGLAAEDLVGRAQPMPYWAPDFLEETLARQRQLIEG QPVPQAFETRFRRSDGSEIEVQVFEAPLIDAGGRHRGWMGSVIDITQAKQAARLARAQDE SLARTGRLVTLGEMASTLAHELNQPLAAIASYAAGGLNLFDQPEPNLTMLRQAFEKMGAQ ARRAGLVIRRVQDFVKKRTPQLAALDLSEVLAEALS ITAPVAREHRVKLASLIEGR I PGV QADRILIEQVLVNLIRNGVEAMAEGPRTGDDLTVRLARAGAAVTIEVMDRGPGISDAVAA SLFDPFTSTKSEGMGMGLNICRS IVEMHHGSLSHGPRAGGGTVFTVTLPVPQEGAPA $>$ P 77510

MLQLNENKQFAFFQRLAFPLRIFLLILVFS IFVIAALAQYFTASFEDYLTLHVRDMAMNQ AKI IASNDSVISAVKTRDYKRLATIANKLQRDTDFDYVVIGDRHS IRLYHPNPEKIGYPM QFTKQGALEKGESYFITGKGSMGMAMRAKTP I FDDDGKVIGVVS IGYLVSKIDSWRAEFL LPMAGVFVVLLGILMLLSWFLAAHIRRQMMGMEPKQIARVVRQQEALFSSVYEGLIAVDP HGYITAINRNARKMLGLSSPGRQWLGKPIVEVVRPADFFTEQIDEKRQDVVANFNGLSVI ANREAIRSGDDLLGAI ISFRSKDEISTLNAQLTQIKQYVESLRTLRHEHLNWMSTLNGLL QMKEYDRVLAMVQGESQAQQQLIDSLREAFADRQVAGLLFGKVQRARELGLKMI IVPGSQ LSQLP PGLDSTEFAAIVGNLLDNAFEASLRSDEGNKIVELFLSDEGDDVVIEVADQGCGV 
PESLRDKIFEQGVSTRADEPGEHGIGLYLIASYVTRCGGVITLEDNDPCGTLFSIYIPKV KPNDSSINPI

$>$ POAFEO

MEFAFYICGLIAILATLRVITHTNPVHALLYLIISLLAISGVFFSLGAYFAGALEI IVYA GAIMVLFVFVVMMLNLGGSEIEQERQWLKPQVWIGPAILSAIMLVVIVYAILGVNDQGID GTPISAKAVGITLFGPYVLAVELASMLLLAGLVVAFHVGREERAGEVLSNRKDDSAKRKT EEHA

$>$ POAEH1

MLSFLWDLASFIVALGVLITVHEFGHFWVARRCGVRVERFS IGFGKALWRRTDKLGTEYV IALI PLGGYVKMLDERAEPVVPELRHHAFNNKSVGQRAA I IAAGPVANF IFA IFAYWLVF I IGVPGVRPVVGEIAANS IAAEAQIAPGTELKAVDGIETPDWDAVRLQLVDKIGDESTTI TVAPFGSDQRRDVKLDLRHWAFEPDKEDPVSSLGIRPRGPQIEPVLENVQPNSAASKAGL QAGDRIVKVDGQPLTQWVTFVMLVRDNPGKSLALEIERQGSPLSLTLIPESKPGNGKAIG FVGIEPKVIPLPDEYKVVRQYGPFNAIVEATDKTWQLMKLTVSMLGKLITGDVKLNNLSG PIS IAKGAGMTAELGVVYYLPFLAL I SVNLGI INLFPLPVLDGGHLLFLAIEKI KGGPVS ERVQDFCYRIGSILLVLLMGLALFNDFSRL

$>$ P69423

SVEDTQPLITHLIELRKRLLNCI IAVIVIFLCLVYFANDIYHLVSAPLIKQLPQGSTMIA TDVASPFFTPIKLTFMVSLILSAPVILYQVWAFIAPALYKHERRLVVPLLVSSSLLFYIG MAFAYFVVFPLAFGFLANTAPEGVQVSTDIASYLSFVMALFMAFGVSFEVPVAIVLLCWM GITSPEDLRKKRPYVLVGAFVVGMLLTPPDVFSQTLLA I PMYCLFEIGVFFSRFYVGKGR NREEENDAEAESEKTEE

$>$ POAFE4

MI PLQHGLILAA ILFVLGLTGLVIRRNLLFMLIGLEIMINASALAFVVAGSYWGQTDGQV MY ILA ISLAAAEAS IGLALLLQLHRRRQNLNIDSVSEMRG

$>$ POAFE 8

MLLPWLILIPFIGGFLCWQTERFGVKVPRWIALITMGLTLALSLQLWLQGGYSLTQSAGI PQWQSEFDMPWI PRFGIS IHLA IDGLSLLMVVLTGLLGVLAVLCSWKEIEKYQGFFHLNL MWILGGVIGVFLAIDMFLFFFFWEMMLVPMYFLIALWGHKASDGKTRITAATKFFIYTQA SGLVMLIAILALVFVHYNATGVWTFNYEELLNTPMSSGVEYLLMLGFFIAFAVKMPVVPL HGWLPDAHSQAPTAGSVDLAGILLKTAAYGLLRFSLPLFPNASAEFAPIAMWLGVIGIFY GAWMAFAQTDIKRLIAYTSVSHMGFVLIAIYTGSQLAYQGAVIQMIAHGLSAAGLF ILCG QLYERIHTRDMRMMGGLWSKMKWLPALSLFFAVATLGMPGTGNFVGEFMILFGSFQVVPV ITVISTFGLVFASVYSLAMLHRAYFGKAKSQIASQELPGMSLRELFMILLLVVLLVLLGF YPQPILDTSHSAIGNIQQWFVNSVTTTRP

$>$ P75916

MNILLSIAITTGILSGIWGWVAVSLGLLSWAGFLGCTAYFACPQGGLKGLAISAATLLSG VVWAMVI IYGSALAPHLEILGYVITGIVAFLMCIQAKQLLLSFVPGTFIGACATFAGQGD WKLVLPSLALGLIFGYAMKNSGLWLAARSAKTAHREQEIKNKA

$>$ P07654

MAMVEMQTTAALAESRRKMQARRRLKNRIALTLSMATMAFGLFWLIWILMSTITRGIDGM SLALFTEMTPPPNTEGGGLANALAGSGLLILWATVFGTPLGIMAGIYLAEYGRKSWLAEV IRFINDILLSAPS IVVGLFVYTIVVAQMEHFSGWAGVIALALLQVPIVIRTTENMLKLVP YSLREAAYALGTPKWKMISAITLKASVSGIMTGILLAIARIAGETAPLLFTALSNQFWST DMMQPIANLPVTI FKFAMSPFAEWQQLAWAGVLI ITLCVLLLNILARVVFAKNKHG $>$ POADK4

MFLDYFALGVLIFVFLVIFYGI I ILHDI PYLIAKKRNHPHADA IHVAGWVSLFTLHVIWP FLWIWATLYRPERGWGMQSHDSSVMQLQQRIAGLEKQLADIKSSSAE

$>033953$

MRVENNNVSGQNLDPEQIDLIDLLVQLWRGKMTI I ISVIVAIVLAIGYLVVAKEKWTSTA IVTQPDVGQIAGYNNAINVIYGSAAPKVSEIQSILIGRFSTTFSALAETLDNQEEPEKLT IEPTVKNQSLPLAVSYVGQSPEAAQKQLAQYIQQVDDQVNDELEKDLKDNIALRMKNLQD SLKTQEVVAQEQKELRIRQIQEALQYANQAQVTKPQIQQTQDVTQDTMFLLGSDALESMV KHEASRPLVFSSTYYQTRQNLLDIESLKVDDLDIHAYRYVMKPTLPIRRDSPKKAITLIL AVLLGGMVGAGIVLGRNALRNYNAK

$>$ POAEH8

MTTQVRKNVMDMF IDGARRGFTIATTNLLPNVVMAFVI IQALKITGLLDWVGHICEPVMA LWGLPGEAATVLLAALMSMGGAVGVAASLATAGALTGHDVTVLLPAMYLMGNPVQNVGRC LGTAEVNAKYYPHI ITVCVINALLS IWVMQ 
$>$ POAC05

MRRLLSVAPVLLWLITPLAFAQLPGITSQPLPGGGQSWSLPVQTLVFITSLTFIPAILLM MTSFTRI I IVFGLLRNALGTPSAPPNQVLLGLALFLTFFIMSPVIDKIYVDAYQPFSEEK ISMQEALEKGAQPLREFMLRQTREADLGLFARLANTGPLQGPEAVPMRILLPAYVTSELK TAFQIGFTIFIPFLI IDLVIASVLMALGMMMVPPATIALPFKLMLFVLVDGWQLLVGSLA QSFYS

$>$ P72292

MRGQRRWAHPFTLIRRLFGNAVFSSLTRRIVFFNLVALVVLVGGIMYLNQFREGLIDARV ESLLTQGEI IAGA ISASASVDTNS ITIDPEKLLELQAGES ITPLPSDEDLEFPI IQERVA PVLRRLISPTRTRARLFDADADLLLDSRHLYSGGQVLRFDLPPVDPESPSLADEFGTWFN RLLQPGDLPLYKEP PGGNGS IYPEVMNALTGVRGAVVRVTEKGELIVSVAVPVQRFRAVL GVLLLSTQAGDIDKIVHAERLAI IRVFGVAALVNVILSLLLSSTIANPLRRLSAAAIRVR RGGAKEREEI PDFSSRQDEIGNLSVALREMTTALYDRIAAIENFAADVSHELKNPLTSLR SAVETLPLARNEESKKRLMDVIQHDVRRLDRLISDISDASRLDAELARADAKKVDLEKLL GDLVEISRQIRGSKKPVLLDFVVDRKDNPRASFIVSGYELRIGQI ITNLIENARSFVPEQ NGRIVVRLTRSRLRCIVYVEDNGPGIQAEDIDRI FERFYTDRPEGEDFGQNSGLGLSISR QIAEAHGGTLRAENIAGKDGRISGARFVLSLPAGPHP

$>$ P39270

MTRTLKPLILNTSALTLTLILIYTGISAHDKLTWLMEVTPVI IVVQLLLATARRYPLTPL LYTLIFLHAI I LMVGGQYTYAKVPVGFEVQEWLGLSRNPYDKLGHFFQGLVPALVAREIL VRGMYVRGRKMVAFLVCCVALAISAMYELIEWWAALAMGQGADDFLGTQGDQWDTQSDMF CALLGALTTVIFLARFHCRQLRRFGLITG

$>$ P37746

MNTNKLSLRRNVIYLAVVQGSNYLLPLLTFPYLVRTLGPENFGIFGFCQATMLYMIMFVE YGFNLTATQS IAKAADSKDKVTS IFWAVIFSKIVLIVITLIFLTSMTLLVPEYNKHAVI I WSFVPALVGNLIYP IWLFQGKEKMKWLTLSS ILSRLA I I PLTF I FVNTKSDIA IAGF IQS SANLVAGI IALA IVVHEGWIGKVTLSLHNVRRSLADGFHVF ISTSAISLYSTGIVI ILGF ISGPTSVGNFNAANTIRNALQGLLNP ITQAIYPRISSTLVLNRVKGVILIKKSLTCLSLI GGAFSLILLLGAS ILVKIS IGPGYDNAVIVLMI ISPLPFLISLSNVYGIQVMLTHNYKKE FSKILIAAGLLSLLLIFPLTTLFKEIGAAITLLATECLVTSLMLMFVRNNKLLVC

$>$ P37748

MIYLVISVFLITAFICLYLKKDIFYPAVCVNI IFALVLLGYEITSDIYAFQLNDATLIFL LCNVLTFTLSCLLTESVLDLNIRKVNNAIYS I PSKKVHNVGLLVISFSMIYICMRLSNYQ FGTSLLSYMNLIRDADVEDTSRNFSAYMQP I I LTTFALF IWSKKFTNTKVSKTFTLLVFI VFIFA I ILNTGKQIVFMVI I SYAF IVGVNRVKHYVYLITAVGVLFSLYMLFLRGLPGGMA YYLSMYLVSPI IAFQEFYFQQVSNSASSHVFWFFERLMGLLTGGVSMSLHKEFVWVGLPT NVYTAFSDYVYISAELSYLMMVIHGCISGVLWRLSRNYISVKIFYSYFIYTFSFIFYHES FMTNISSWIQITLCIIVFSQFLKAQKIK

$>$ POAGCO

MLAFLNQVRKPTLDLPLEVRRKMWFKPFMQSYLVVF IGYLTMYLIRKNFNIAQNDMISTY GLSMTQLGMIGLGFS ITYGVGKTLVSYYADGKNTKQFLPFMLILSAICMLGFSASMGSGS VSLFLMIAFYALSGFFQSTGGSCSYSTITKWTPRRKRGTFLGFWNI SHNLGGAGAAGVAL FGANYLFDGHVIGMF I FPS I IALIVGF IGLRYGSDSPESYGLGKAEELFGEEISEEDKET ESTDMTKWQI FVEYVLKNKVIWLLCFANI FLYVVRIGIDQWSTVYAFQELKLSKAVAIQG FTLFEAGALVGTLLWGWLSDLANGRRGLVACIALALI IATLGVYQHASNEYIYLASLFAL GFLVFGPQLLIGVAAVGFVPKKAIGAADGI KGTFAYLIGDSFAKLGLGMIADGTPVFGLT GWAGTFAALDIAAIGCICLMAIVAVMEERKIRREKKIQQLTVA

$>$ P45768

MTKVLLSHPPRPASHNSSRAMVWVRKNLFSSWSNSLLTIGCIWLMWELI PPLLNWAFLQA NWVGSTRADCTKAGACWVF I HERFGQFMYGLYPHDQRWR INLALLIGLVS IAPMFWKILP HRGRYIAAWAVIYPLIVWWLMYGGFFALERVETRQWGGLTLTLI IASVGIAGALPWGILL ALGRRSHMPIVRILSVIFIEFWRGVPLITVLFMSSVMLPLFMAEGTS IDKLIRALVGVIL FQSAYVAEVVRGGLQALPKGQYEAAESLALGYWKTQGLVILPQALKLVI PGLVNTI IALF KDTSLVI I I GLFDLFSSVQQATVDPAWLGMSTEGYVFAALIYWI FCFSMSRYSQYLEKRF NTGRTPH

$>$ P23910

MKKVILSLALGTFGLGMAEFGIMGVLTELAHNVGIS I PAAGHMI SYYALGVVVGAPI IAL FSSRYSLKHILLFLVALCVIGNAMFTLSSSYLMLAIGRLVSGFPHGAFFGVGAIVLSKI I KPGKVTAAVAGMVSGMTVANLLGI PLGTYLSQEFSWRYTFLLIAVFNIAVMASVYFWVPD 
IRDEAKGNLREQFHFLRSPAPWLIFAATMFGNAGVFAWFSYVKPYMMFISGFSETAMTFI MMLVGLGMVLGNMLSGR ISGRYSPLR IAAVTDF I IVLALLMLFFCGGMKTTSLI FAF I CC AGLFALSAPLQILLLQNAKGGELLGAAGGQIAFNLGSAVGAYCGGMMLTLGLAYNYVALP AALLSFAAMSSLLLYGRYKRQQAADTPVLAKPLG

$>$ P69432

MNNLI ITTRQS PVRLLVDYVATTILWTLFALFIFLFAMDLLTGYYWQSEARSRLQFYFLL AVANAVVLIVWALYNKLRFQKQQHHAAYQYTPQEYAESLA I PDELYQQLQKSHRMSVHFT SQGQIKMVVSEKALVRA

$>Q 05490$

MAQADRPARGGLAARPMRGASFALAGLVACAACAAVVLWLRPAAPSPAPAGAVAGGPAAG VPAAASGAAEAAMPLPAALPGALAGSHAPRLPLAAGGRLARTRAVREFFDYCLTAQGELT PAALDALVRREIAAQLDGSPAQAEALGVWRRYRAYFDALAQLPGDGAVLGDKLDPAAMQL ALDQRAALADRTLGEWAEPFFGDEQRRQRHDLERIRIANDTTLSPEQKAARLAALDAQLT PDERAQQAALHAQQDAVTKIADLQKAGATPDQMRAQIAQTLGPEAAARAAQMQQDDEAWQ TRYQAYAAERDRIAAQGLAPQDRDARIAQLRQQTFTAPGEAIRAASLDRG

$>$ P16552

MNSASTHKNTDFWIFGLFFFLYFFIMATCFPFLPVWLSDVVGLSKTDTGIVFSCLSLFAI SFQPLLGVISDRLGLKKNLIWS ISLLLVFFAPFFLYVFAPLLHLNIWAGALTGGVFIGFV FSAGAGAIEAYIERVSRSSGFEYGKARMFGCLGWALCATMAGILFNVDPSLVFWMGSGGA LLLLLLLYLARPSTSQTAMVMNALGANSSLISTRMVFSLFRMRQMWMFVLYTIGVACVYD VFDQQFAIFFRSFFDTPQAGI KAFGFATTAGEI CNAI IMFCTPWI INRIGAKNTLLVAGG IMTIRITGSAFATTMTEVVILKMLHALEVPFLLVGAFKYITGVFDTRLSATVYLIGFQFS KQLAAILLSTFAGHLYDRMGFQNTYFVLGMIVLTVTVISAFTLSSSPGIVHPSVEKAPVA HSEIN

$>$ POADL1

MLKWSAFPLKHATGNTMSEFIAENRGADAITRPNWSAVFSVAFCVACLI IVEFLPVSLLT PMAQDLGISEGVAGQSVTVTAFVAMFASLFITQTIQATDRRYVVILFAVLLTLSCLLVSF ANSFSLLLIGRACLGLALGGFWAMSASLTMRLVPPRTVPKALSVIFGAVS IALVIAAPLG SFLGELIGWRNVFNAAAVMGVLCIFWI I KSLPSLPGEPSHQKQNTFRLLQRPGVMAGMIA IFMSFAGQFAFFTYIRPVYMNLAGFGVDGLTLVLLSFGIASFIGTSLSSFILKRSVKLAL AGAPLILAVSALVLTLWGSDKIVATGVAI IWGLTFALVPVGWSTWITRSLADQAEKAGS I QVAVIQLANTCGAAIGGYALDNIGLTSPLMLSGTLMLLTALLVTAKVKMKKS $>$ P69367

MSRVSQARNLGKYFLLIDNMLVVLGFFVVFPLISIRFVDQMGWAAVMVGIALGLRQFIQQ GLGIFGGAIADRFGAKPMIVTGMLMRAAGFATMGIAHEPWLLWF SCLLSGLGGTLFDPPR SALVVKLIRPQQRGRFF SLLMMQDSAGAVIGALLGSWLLQYDFRLVCATGAVLFVLCAAF NAWLLPAWKLSTVRTPVREGMTRVMRDKRFVTYVLTLAGYYMLAVQVMLMLPIMVNDVAG APSAVKWMYAIEACLSLTLLYPIARWSEKHFRLEHRLMAGLLIMSLSMMPVGMVSGLQQL FTLICLFYIGS I IAEPARETLSASLADARARGSYMGFSRLGLAIGGAIGYIGGGWLFDLG KSAHQPELPWMMLGI IGIFTFLALGWQFSQKRAARRLLER

$>$ P23849

MNTSHVRVVTHMCGFLVWLYSLSMLPPMVVALFYKEKSLFVFFITFVIFFCIGGGAWYTT KKSGIQLRTRDGF I I IVMFWILFSVISAFPLWIDSELNLTF IDALFEGVSGITTTGATVI DDVSSLPRAYLYYRSQLNF IGGLGVIVLAVAVLPLLGIGGAKLYQSEMPGPFKDDKLTPR LADTSRTLWITYSLLGIACIVCYRLAGMPLFDAI CHGISTVSLGGFSTHSES IGYFNNYL VELVAGSFSLLSAFNFTLWYIVISRKTIKPLIRDIELRFFLLIALGVI IVTSFQVWHIGM YDLHGSF IHSFFLASSMLTDNGLATQDYASWPTHTIVFLLLSSFFGGCIGSTCGGIKSLR FLILFKQSKHEINQLSHPRALLSVNVGGKIVTDRVMRSVWSFFFLYTLFTVFF ILVLNGM GYDFLTSFATVAACINNMGLGFGATASSFGVLNDIAKCLMCIAMILGRLEIYPVI ILFSG FFWRS

$>$ P 64423

MEAIKGSDVNVPDAVFAWMLDGRGGVKPLENTDVIDEAHPCWLHLNYVHHDSAQWLATTP LLPNNVRDALAGESTRPRVSRLGEGTLITLRCINGSTDERPDQLVAMRVYMDGRLIVSTR QRKVLALDDVVSDLEEGTGPTDCGGWLVDVCDALTDHSSEFIEQLHDKI IDLEDNLLDQQ I PPRGFLALLRKQLIVMRRYMAPQRDVYARLASERLPWMSDDQRRRMQDIADRLGRGLDE IDACIARTGVMADEIAQVMQENLARRTYTMSLMAMVFLPSTFLTGLFGVNLGGI PGGGWQ FGFSIFCILLVVLIGGVALWLHRSKWL

$>$ P75785

MNLTLKESLVTRSRVFSPWTAFYFLQSLLINLGLGYPFSLLYTAAFTAILLLLWRTLPRV 
QKVLVGVSSLVAACYFPFAQAYGAPNFNTLLALHSTNMEESTEILTIFPWYSYLVGLFIF ALGVIAIRRKKENEKARWNTFDSLCLVFSVATFFVAPVQNLAWGGVFKLKDTGYPVFRFA KDVIVNNNEVIEEQERMAKLSGMKDTWTVTAVKPKYQTYVVVIGESARRDALGAFGGHWD NTPFASSVNGLIFADYIAASGSTQKSLGLTLNRVVDGKPQFQDNFVTLANRAGFQTWWFS NQGQIGEYDTAIAS IAKRADEVYFLKEGNFEADKNTKDEALLDMTAQVLAQEHSQPQLIV LHLMGSHPQACDRTQGKYETFVQSKETSCYLYTMTQTDDLLRKLYDQLRNSGSSFSLVYF SDHGLAFKERGKDVQYLAHDDKYQQNFQVPFMVISSDDKAHRVIKARRSANDFLGFFSQW TGIKAKEINIKYPFISEKKAGPIYITNFQLQKVDYNHLGTDIFDPKP

$>$ P75788

MSLPFLRTLQGDRFFQLLILVGIGLSFFVPFAPKSWPAAIDWHTI ITLSGLMLLTKGVEL SGYFDVLGRKMVRRFATERRLAMFMVLAAALLSTFLTNDVALFIVVPLTITLKRLCEIPV NRLI I FEALAVNAGSLLTP I GNPQNILIWGRSGLSFAGF IAQMAPLAGAMMLTLLLLCWC CFPGKAMQYHTGVQTPEWKPRLVWSCLGLYIVFLTALEFKQELWGLVIVAAGFALLARRV VLSVDWTLLLVFMAMF IDVHLLTQLPALQGVLGNVSHLSEPGLWLTAIGLSQVISNVPST ILLLNYVPPSLLLVWAVNVGGFGLLPGSLANLIALRMANDRR IWWRFHLYS I PMLLWAAL VGYVLLVILPAN

$>$ POAA73

MKQQAGIGILLALTTA I CWGALP IAMKQVLEVMEP PTIVFYRFLMAS IGLGAILAVKKRL PPLRVFRKPRWLILLAVATAGLFGNF ILFSSSLQYLSPTASQVIGQLSPVGMMVASVF IL KEKMRSTQVVGALMLLSGLVMFFNTSLVEIFTKLTDYTWGVIFGVGAATVWVSYGVAQKV LLRRLASPQILFLLYTLCTIALFPLAKPGVIAQLSHWQLACLIFCGLNTLVGYGALAEAM ARWQAAQVSAI ITLTPLFTLFFSDLLSLAWPDFFARPMLNLLGYLGAFVVVAGAMYSAIG HRIWGGLRKHTTVVSQPRAGE

$>$ Q8RK01

MNAALSQWKDAHAARLRDYSAVRVSGRVSAVRGILLECKI PAAKVGDLCEVSKADGSFLL AEIVGFTQECTLLSALGAPDGIQVGAP IRPLGIAHR IGVDDSLLGCVLDGFGRPLLGDCL GAFAGPDDRRETLPVIADALPPTQRPRITNALPTGVRAIDSAILLGEGQRVGLFAGAGCG KTTLMAELARNMGCDVIVFGLIGERGRELREFLDHELDETLRRRSVLVCATSDRSSMERA RAAFTATAIAEAFRARGQKVLLLLDSLTRFARAQREIGIASGEPLGRGGLPPSVYTLLPR LVERAGMSENGSITALYTVLIEQDSMNDPVADEVRSLLDGHIVLSRKLAERGHYPAIDVS AS ISR ILSNVTGREHQRANNRLRQLLAAYKQVEMLLRLGEYQAGADPVTDCAVQLNDDIN EFLRQDLREPVPLQETLDGLLRLTSRLPE

$>$ P39284

MSARMFVLCCIWFIVAFLWITITSALDKEWMIDGRGINNVCDVLMYLEEDDTRDVGVIMT LPLFFPFLWFALWRKKRGWFMYATALAIFGYWLWQFFLRY

$>$ P37683

MDLLI ILTYVAFAWAMFKI FKI PVNKWT I PTAALGGIF IVSGLILLMNYNHPYTFKAQKA VISIPVVPQVTGVVIEVTDKKNTLIKKGEVLFRLDPTRYQARVDRLMADIVTAEHKQRAL GAELDEMAANTQQAKATRDKFAKEYQRYARGSQAKVNPFSERDIDVARQNYLAQEASVKS SAAEQKQIQSQLDSLVLGEHSQIASLKAQLAEAKYNLEQTIVRAPSDGYVTQVLIRPGTY AASLPLRPVMVF I PDQKRQIVAQFRQNSLLRLAPGDDAEVVFNALPGKVF SGKLAAISPA VPGGAYQSTGTLQTLNTAPGSDGVIATIELDEHTDLSALPDGIYAQVAVYSDHFSHVSVM RKVLLRMTSWVHYLYLDH

$>$ P2 7125

MSNAITMGIFWHLIGAASAACFYAPFKKVKKWSWETMWSVGGIVSWI ILPWAISALLLPN FWAYYSSFSLSTRLPVFLFGAMWGIGNINYGLTMRYLGMSMGIGIAIGITLIVGTLMTPI INGNFDVLISTEGGRMTLLGVLVALIGVGIVTRAGQLKERKMGIKAEEFNLKKGLVLAVM CGIFSAGMSFAMNAAKPMHEAAAALGVDPLYVALPSYVVIMGGGA I INLGFCF IRLAKVK DLSLKADFSLAKSLI IHNVLLSTLGGLMWYLQFFFYAWGHARI PAQYDYISWMLHMSFYV LCGGIVGLVLKEWNNAGRRPVTVLSLGCVVI IVAANIVGIGMAN

$>$ POAEJO

MQQQKPLEGAQLVIMTIALSLATFMQVLDSTIANVAI PTIAGNLGSSLSQGTWVITSFGV ANAIS I PLTGWLAKRVGEVKLFLWSTIAFAIASWACGVSSSLNMLIFFRVIQGIVAGPLI PLSQSLLLNNYPPAKRS IALALWSMTVIVAP I CGP ILGGYISDNYHWGW I FF INVPIGVA VVLMTLQTLRGRETRTERRRIDAVGLALLVIGIGSLQIMLDRGKELDWFSSQEI I ILTVV AVVAICFLIVWELTDDNPIVDLSLFKSRNFTIGCLCISLAYMLYFGAIVLLPQLLQEVYG YTATWAGLASAPVGI I PVILSPI I GRFAHKLDMRRLVTFSF IMYAVCFYWRAYTFEPGMD FGASAWPQFIQGFAVACFFMPLTTITLSGLPPERLAAASSLSNFTRTLAGSIGTSITTTM WTNRESMHHAQLTESVNPFNPNAQAMYSQLEGLGMTQQQASGW IAQQITNQGLI ISANE I 
FWMSAGIFLVLLGLVWFAKPPFGAGGGGGGAH

$>$ P32678

MHSTEVQAKPLFSWKALGWALLYFWFFSTLLQAI IYISGYSGTNGIRDSLLFSSLWLI PV FLFPKRIKI IAAVIGVVLWAASLAALCYYVIYGQEFSQSVLFVMFETNTNEASEYLSQYF SLKIVLIALAYTAVAVLLWTRLRPVYIPKPWRYVVSFALLYGLILHPIAMNTFIKNKPFE KTLDNLASRMEPAAPWQFLTGYYQYRQQLNSLTKLLNENNALPPLANFKDESGNEPRTLV LVIGESTQRGRMSLYGYPRETTPELDALHKTDPNLTVFNNVVTSRPYTIEILQQALTFAN EKNPDLYLTQPSLMNMMKQAGYKTFWITNQQTMTARNTMLTVFSRQTDKQYYMNQQRTQS AREYDTNVLKPFQEVLNDPAPKKLI IVHLLGTHI KYKYRYPENQGKFDGNTDHVPPGLNA EELESYNDYDNANLYNDHVVASL I KDFKAANPNGFLVYFSDHGEEVYDTPPHKTQGRNED NPTRHMYTIPFLLWTSEKWQATHPRDFSQDVDRKYSLAELIHTWSDLAGLSYDGYDPTRS VVNPQFKETTRWIGNPYKKNALIDYDTLPYGDQVGNQ

$>$ P24586

MIKIENLTKSYRTPTGRHYVFKDLNIEIPSGKSVAFIGRNGAGKSTLLRMIGGIDRPDSG KIITNKTISWPVGLAGGFQGSLTGRENVKFVARLYAKQEELKEKIEFVEEFAELGKYFDM PIKTYSSGMRSRLGFGLSMAFKFDYYIVDEVTAVGDARFKEKCAQLFKERHKESSFLMVS HSLNSLKEFCDVAIVFKNSYI I GYYENVQSGIDEYKMYQDLDIE

$>087656$ MSRKREMPDGGAKSVLSDLRFGRFVGRIRRSRHPALLLLALFVAACWLTWVNFSVALPRS QWQQA IWSPDIDI IEQMIFHYSQLPRLAISLLVGAGLGLVGVLFQQVLRNPLAEPTTLGV ATGAQLGITVTTLWAI PGALTTQFAALTGACIVGALVFGVAWGKRLSPVTLILAGLVVSL YCGAINQLLVIFHHDQLQSMFLWSTGTLTQTDWSGVQRLWPQLLGGVMLTLLLLRPMTLM GLDDGVARNLGLALSLARLAALSLA IVLSALLVNAVG I I GF I GLFAPLLAKMLGARRLLA RLMLAPLIGAL ILWLSDQ I I LWLTRVWMEVSTGSVTALIGAPLLLWLLPRLKSMSAPDMN ASDRVAAERRHVLAFAVAGGALLLLATWVALSFGRDAHGWTWASGTLLEELMPWRWPRIL AALMAGVMLAVAGCI IQRLTGNPMAS PEVLGISSGAAFGVVLMLFLVPGNAFGWLLPAGS LGAAATLL I IMIAAGRGGF S PQRMLLAGMALSTAFTMLLMMLQASGDPRMAEVLTWLSGS TYNATGGQVTRTAIVMVILLAIVPLCRRWLTILPLGGDAARAVGMALTPSR IALLALAAC LTATATMTIGPLSFVGLMAPHIARMLGFRRTMPHMVISALAGGVLLVFADWCGRMALFPY QIPAGLLSSFIGAPYFIYLLRKQSR

$>$ P64432

MPVFALLALVAYSVSLALIVPGLLQKNGGWRRMAI ISAVIALVCHAIALEARILPDGDSG QNLSLLNVGSLVSLMICTVMTIVASRNRGWLLLPIVYAFALINLALATFMPNEYITHLEA TPGMLVHIGLSLFSYATLI IAALYALQLAWIDYQLKNKKLAFNQEMP PLMS IERKMFHIT QIGVVLLTLTLCTGLFYMHNLFSMENIDKAVLS IVAWFVYIVLLWGHYHEGWRGRRVVWF NVAGAVILTLAYFGSRIVQQLIS

$>$ P11551

GNTS IQTQSYRAVDKDAGQSRSYI I PFALLCSLFFLWAVANNLNDILLPQFQQAFTLTNF QAGLIQSAFYFGYFI I P I PAGILMKKLSYKAGI ITGLFLYALGAALFWPAAE IMNYTLFL VGLFI IAAGLGCLETAANPFVTVLGPESSGHFRLNLAQTFNSFGA I IAVVFGQSLILSNV PHQSQDVLDKMSPEQLSAYKHSLVLSVQTPYMI IVAIVLLVALLIMLTKF PALQSDNHSD AKQGSFSASLSRLARIRHWRWAVLAQFCYVGAQTACWSYLIRYAVEEIPGMTAGFAANYL TGTMVCFFIGRFTGTWLISRFAPHKVLAAYALIAMALCLISAFAGGHVGLIALTLCSAFM SIQYPTIFSLGI KNLGQDTKYGSSF IVMT I IGGGIVTPVMGFVSDAAGN I PTAELI PALC FAVIFIFARFRSQTATN

$>$ POAC23

MKADNPFDLLLPAAMAKVAEEAGVYKATKHPLKTFYLAITAGVFISIAFVFYITATTGTG TMPFGMAKLVGGI CFSLGLILCVVCGADLFTSTVLIVVAKASGR ITWGQLAKNWLNVYFG NLVGALLFVLLMWLSGEYMTANGQWGLNVLQTADHKVHHTF IEAVCLGILANLMVCLAVW MSYSGRSLMDKAF IMVLPVAMFVASGFEHS IANMFMI PMGIVIRDFASPEFWTAVGSAPE NFSHLTVMNF ITDNLI PVTIGNI IGGGLLVGLTYWVIYLRENDHH

$>$ POA8R7

MTEPLKPRIDFDGPLEVDQNPKFRAQQTFDENQAQNFAPATLDEAQEEEGQVEAVMDAAL RPKRSLWRKMVMGGLALFGASVVGQGVQWTMNAWQTQDWVALGGCAAGALI I GAGVGSVV TEWRRLWRLRQRAHERDEARDLLHSHGTGKGRAFCEKLAQQAGIDQSHPALQRWYAS IHE TQNDREVVSLYAHLVQPVLDAQARREISRSAAESTLMIAVSPLALVDMAFIAWRNLRLIN RIATLYGIELGYYSRLRLFKLVLLNIAFAGASELVREVGMDWMSQDLAARLSTRAAQGIG AGLLTARLGIKAMELCRPLPWIDDDKPRLGDFRRQLIGQVKETLQKGKTP $>$ P 64439 
MKHKQRWAGA I CCFVLFIVVCLFLATHMKGAFRAAGHPEIGLLFFILPGAVASFFSQRRE VLKPLFGAMLAAPCSMLIMRLFFSPTRSFWQELAWLLSAVFWCALGALCFLF ISSLFKPQ HRKNQ

$>$ P5 4745

MVLFYRAHWRDYKNDQVRIMMNLTTLTHRDALCLNARFTSREEAIHALTQRLAALGKISS TEQFLEEVYRRESLGPTALGEGLAVPHGKTAAVKEAAFAVATLSEPLQWEGVDGPEAVDL VVLLAIPPNEAGTTHMQLLTALTTRLADDEIRAR IQSATTPDELLSALDDKGGTQPSASF SNAPTIVCVTACPAGIAHTYMAAEYLEKAGRKLGVNVYVEKQGANGIEGRLTADQLNSAT ACIFAAEVAIKESERFNGI PALSVPVAEP IRHAEALIQQALTLKRSDETRTVQQDTQPVK SVKTELKQALLSGISFAVPLIVAGGTVLAVAVLLSQIFGLQDLFNEENSWLWMYRKLGGG LLGILMVPVLAAYTAYSLADKPALAPGFAAGLAANMIGSGFLGAVVGGLIAGYLMRWVKN HLRLSSKFNGFLTFYLYPVLGTLGAGSLMLFVVGEPVAWINNSLTAWLNGLSGSNALLLG AILGFMCSFDLGGPVNKAAYAFCLGAMANGVYGPYAIFASVKMVSAFTVTASTMLAPRLF KEFEIETGKSTWLLGLAGITEGAI PMAIEDPLRVIGSFVLGSMVTGAIVGAMNIGLSTPG AGIFSLFLLHDNGAGGVMAAIGWFGAALVGAA ISTA I LLMWRRHAVKHGNYLTDGVMP $>Q 08120$

MFQSFFPKPKLFFISSAVWSLLAVLAWYAGGRDIGAYLGLPPLPPGQEPVIGVSVFWSTP FLWFYIYYAVVAGLFAAFWFAYSPHRWQYWSVLGTALI I FNTYFSVQVSVAINAWYGPFY DLIQQALARTAPVTAGQLYSGMIGFSGIAFVAVTVGVLNLFFVSHYIFRWRTAMNEFYVA HWPRLRHVEGASQRVQEDTMRFSSTVERLGVGLVSS IMTLIAFLPVLFKF SEQVNVLPIV GEIPHALVWAAVFWSVFGTVFLAAVGI KLPGLEFRNQRVEAAYRKELVYGEDHEDRADP I TLAQLFDNVRRNYFRLYFHYMYFNIARIFYLQADNLFGTFVLVPAIVAGKLTLGVMNQVL NVFGQVRESFQYLVNSWTTIVELLS IYKRLKAFESVLVDEPLPEIDRQF IDAGGKEELAL $>$ P 77610

MSKHDTDTSDQHAAKRRWLNAHEEGYHKAMGNRQVQMIAIGGAIGTGLFLGAGARLQMAG PALALVYLICGLF SFF ILRALGELVLHRP SSGSFVSYAREFLGEKAAYVAGWMYF INWAM TGIVDITAVALYMHYWGAFGGVPQWVFALAALTIVGTMNMIGVKWFAEMEFWFALIKVLA IVTFLVVGTVFLGSGQPLDGNTTGFHLITDNGGFFPHGLLPALVLIQGVVFAFAS IEMVG TAAGECKDPQTMVPKAINSVIWRIGLFYVGSVVLLVMLLPWSAYQAGQSPFVTFFSKLGV PYIGS IMNIVVLTAALSSLNSGLYCTGR ILRSMAMGGSAPSFMAKMSRQHVPYAGILATL VVYVVGVFLNYLVPSRVFEIVLNFASLGI IASWAFI IVCQMRLRKAIKEGKAADVSFKLP GAPFTSWLTLLFLLSVLVLMAFDYPNGTYTIAALP I IGILLVIGWFGVRKRVAEIHSTAP VVEEDEEKQEIVFKPETAS

$>$ P2 6406

MDNIDNKYNPQLCKIFLAISDLIFFNLALWFSLGCVYFIFDQVQRF I PQDQLDTRVITHF ILSVVCVGWFWIRLRHYTYRKPFWYELKEIFRTIVIFAIFDLALIAFTKWQFSRYVWVFC WTFALILVPFFRALTKHLLNKLGIWKKKTI ILGSGQNARGAYSALQSEEMMGFDVIAFFD TDASDAE INMLPVI KDTEI IWDLNRTGDVHY I LAYEYTELEKTHFWLRELSKHHCRSVTV VPSFRGLPLYNTDMSFIFSHEVMLLRIQNNLAKRSSRFLKRTFDIVCS IMILI IASPLMI YLWYKVTRDGGPAIYGHQRVGRHGKLFPCYKFRSMVMNSQEVLKELLANDPIARAEWEKD FKLKNDPRITAVGRFIRKTSLDELPQLFNVLKGDMSLVGPRPIVSDELERYCDDVDYYLM AKPGMTGLWQVSGRNDVDYDTRVYFDSWYVKNWTLWNDIAILFKTAKVVLRRDGAY $>$ P17443

SGQGKRLMVMAGGTGGHVFPGLAVAHHLMAQGWQVRWLGTADRMEADLVPKHGIEIDFIR ISGLRGKGIKALIAAPLRI FNAWRQARA IMKAYKPDVVLGMGGYVSGPGGLAAWSLGI PV VLHEQNGIAGLTNKWLAKIATKVMQAFPGAFPNAEVVGNPVRTDVLALPLPQQRLAGREG PVRVLVVGGSQGARILNQTMPQVAAKLGDSVTIWHQSGKGSQQSVEQAYAEAGQPQHKVT EFIDDMAAAYAWADVVVCRSGALTVSEIAAAGLPALFVPFQHKDRQQYWNALPLEKAGAA KI IEQPQLSVDAVANTLAGWSRETLLTMAERARAAS I PDATERVANEVSR

$>$ POAGE 4

MTTQRSPGLFRRLAHGSLVKQILVGLVLGILLAWISKPAAEAVGLLGTLFVGALKAVAPI LVLMLVMAS IANHQHGQKTNIRP ILFLYLLGTFSAALAAVVFSFAFPSTLHLSSSAGDIS PPSGIVEVMRGLVMSMVSNP IDALLKGNYIGILVWAIGLGFALRHGNETTKNLVNDMSNA VTFMVKLVIRFAPIGIFGLVSSTLATTGFSTLWGYAQLLVVLVGCMLLVALVVNPLLVWW KIRRNPF PLVLLCLRESGVYAFFTRSSAANI PVNMALCEKLNLDRDTYSVSI PLGATINM AGAAITITVLTLAAVNTLGI PVDLPTALLLSVVASLCACGASGVAGGSLLLI PLACNMFG ISNDIAMQVVAVGFI IGVLQDSCETALNSSTDVLFTAAACQAEDDRLANSALRN $>$ P08550

MVWIDYAI IAVIAFSSLVSLIRGFVREALSLVTWGCAFFVASHYYTYLSVWFTGFEDELV 
RNGIAIAVLFIATLIVGAIVNFVIGQLVEKTGLSGTDRVLGVCFGALRGVLIVAAILFFL DSFTGVSKSEDWSKSQLI PQFSFIIRCFFDYLQSSSSFLPRA

$>$ P2 6266

MSSLNIKQGSDAHFPDYPLASPSNNEIDLLNLISVLWRAKKTVMAVVFAFACAGLLISFI LPQKWTSAAVVTPPEPVQWQELEKSFTKLRVLDLDIKIDRTEAFNLFIKKFQSVSLLEEY LRSSPYVMDQLKEAKIDELDLHRAIVALSEKMKAVDDNASKKKDEPSLYTSWTLSFTAPT SEEAQTVLSGYIDYISTLVVKESLENVRNKLEIKTQFEKEKLAQDRIKTKNQLDANIQRL NYSLDIANAAGIKKPVYSNGQAVKDDPDFS ISLGADGIERKLEIEKAVTDVAELNGELRN RQYLVEQLTKAHVNDVNFTPFKYQLSPSLPVKKDGPGKAI IVILSALIGGMVACGGVLLR YAMASRKQDAMMADHLV

$>$ POAEK4

GFLSGKRILVTGVASKLSIAYGIAQAMHREGAELAFTYQNDKLKGRVEEFAAQLGSDIVL QCDVAEDASIDTMFAELGKVWPKFDGFVHSIGFAPGDQLDGDYVNAVTREGFKIAHDISS YSFVAMAKACRSMLNPGSALLTLSYLGAERA I PNYNVMGLAKASLEANVRYMANAMGPEG VRVNAISAGPIRTLAASGI KDFRKMLAHCEAVTPIRRTVTIEDVGNSAAFLCSDLSAGIS GEVVHVDGGFS IAAMNELELK

$>$ POAD03

MALNTPQITPTKKITVRAIGEELPRGDYQRCPQCDMLFSLPEINSHQSAYCPRCQAKIRD GRDWSLTRLAAMAFTMLLLMPFAWGEPLLHIWLLGIRIDANVMQGIWQMTKQGDAITGSM VFFCVIGAPLILVTSIAYLWFGNRLGMNLRPVLLMLERLKEWVMLDIYLVGIGVAS IKVQ DYAHIQAGVGLFSFVALVILTTVTLSHLNVEELWERFYPQRPATRRDEKLRVCLGCHFTG YPDQRGRCPRCHI PLRLRRRHSLQKCWAALLAS IVLLLPANLLP I S I IYLNGGRQEDT IL SGIMSLASSNIAVAGIVFIAS ILVPFTKVIVMFTLLLS IHFKCQQGLRTRILLLRMVTWI GRWSMLDLFVISLTMSLINRDQILAFTMGPAAFYFGAAVILTILAVEWLDSRLLWDAHES GNARFDD

$>$ P10906

MIENRPWLTIFSHTMLILGIAVILFPLYVAFVAATLDKQAVYAAPMTLI PGTHLLENIHN IWVNGVGTNSAPFWRMLLNSFVMAFS ITLGKITVSMLSAFAIVWFRFPLRNLFFWMIFIT LMLPVEVRIFPTVEVIANLQMLDSYAGLTLPLMASATATFLFRQFFMTLPDELVEAARID GASPMRFFCDIVFPLSKTNLAALFVITFIYGWNQYLWPLLI ITDVDLGTTVAGIKGMIAT GEGTTEWNSVMVAMLLTLI PPVVIVLVMQRAFVRGLVDSEK

$>$ P0A8S5

MISTVALFWALCVVCIVNMARYFSSLRALLVVLRNCDPLLYQYVDGGGFFTSHGQPNKQV RLVWYIYAQRYRDHHDDEFIRRCERVRRQFILTSALCGLVVVSLIALMIWH

$>$ POABT8

MSAAGKSNPLAISGLVVLTLIWSYSWIFMKQVTSYIGAFDFTALRCIFGALVLFIVLLLR GRGMRPTPFKYTLAIALLQTCGMVGLAQWALVSGGAGKVAILSYTMPFWVVIFAALFLGE RLRRGQYFAILIAAFGLFLVLQPWQLDFSSMKSAMLAILSGVSWGASAIVAKRLYARHPR VDLLSLTSWQMLYAALVMSVVALLVPQREIDWQPTVFWALAYSAILATALAWSLWLFVLK NLPAS IASLSTLAVPVCGVLFSWWLLGENPGAVEGSGIVLIVLALALVSRKKKEAVSVKR $>$ P0AB 67

MSGGLVTAAYIVAAILFIFSLAGLSKHETSRQGNNFGIAGMAIALIATIFGPDTGNVGWI LLAMVIGGAIGIRLAKKVEMTEMPELVAILHSFVGLAAVLVGFNSYLHHDAGMAP ILVNI HLTEVFLGIFIGAVTFTGSVVAFGKLCGKISSKPLMLPNRHKMNLAALVVSFLLLIVFVR TDSVGLQVLALLIMTAIALVFGWHLVAS I GGADMPVVVSMLNSYSGWAAAAAGFMLSNDL LIVTGALVGSSGAILSYIMCKAMNRSFISVIAGGFGTDGSSTGDDQEVGEHREITAEETA ELLKNSHSVI ITPGYGMAVAQAQYPVAEITEKLRARGINVRFGIHPVAGRLPGHMNVLLA EAKVPYDIVLEMDEINDDFADTDTVLVIGANDTVNPAAQDDPKSPIAGMPVLEVWKAQNV IVFKRSMNTGYAGVQNPLFFKENTHMLFGDAKASVDAILKAL $>$ P2 6273

MAKFYKIWLIFDPRRVFVAQGVFLFLLAAMIHLVVLSSGLNWFEAAAAVG

$>Q 05807$

MMKVIFTSNRFIDFLIRLLITAIVISPVI IWSWDTVKETTADGMLAAAFVILYSGVLLFI LYFCFSALTDLQKPDERKSDERNEDE

$>$ POAGF4

MNTQYNSSYIFSITLVATLGGLLFGYDTAVISGTVESLNTVFVAPQNLSESAANSLLGFC VASALIGCI IGGALGGYCSNRFGRRDSLKIAAVLFFISGVGSAWPELGFTS INPDNTVPV YLAGYVPEFVIYRI IGGIGVGLASMLSPMYIAELAPAHIRGKLVSFNQFAI I FGQLLVYC VNYFIARSGDASWLNTDGWRYMFASECI PALLFLMLLYTVPESPRWLMSRGKQEQAEGIL 
RKIMGNTLATQAVQEIKHSLDHGRKTGGRLLMFGVGVIVIGVMLS I FQQFVGINVVLYYA PEVFKTLGASTDIALLQTI IVGVINLTFTVLA IMTVDKFGRKPLQI I GALGMA I GMF SLG TAFYTQAPGIVALLSMLFYVAAFAMSWGPVCWVLLSEIFPNAIRGKALAIAVAAQWLANY FVSWTFPMMDKNSWLVAHFHNGFSYWIYGCMGVLAALFMWKFVPETKGKTLEELEALWEP ETKKTQQTATL

$>$ P15928

SATASTATQPKPLEWLNRLRANPRIPLIVAGSAAVAIVVAMVLWAKTPDYRTLFSNLSDQ DGGAIVAQLTQMN I PYRFANGSGAIEVPADKVHELRLRLAQQGLPKGGAVGFELLDQEKF GISQFSEQVNYQRALEGELARTIETLGPVKSARVHLAMPKPSLFVREQKSPSASVTVTLE PGRALDEGQISAVVHLVSSAVAGLPPGNVTLVDQSGHLLTQSNTSGRDLNDAQLKFANDV ESRIQRRIEAILSPIVGNGNVHAQVTAQLDFANKEQTEEHYSPNGDASKATLRSRQLNIS EQVGAGYPGGVPGALSNQPAPPNEAPIATPPTNQQNAQNTPQTSTSTNSNSAGPRSTQRN ETSNYEVDRTIRHTKMNVGDIERLSVAVVVNYKTLADGKPLPLTADQMKQIEDLTREAMG FSDKRGDTLNVVNSPFSAVDNTGGELPFWQQQSF IDQLLAAGRWLLVLVVAWILWRKAVR PQLTRRVEEAKAAQEQAQVRQETEEAVEVRLSKDEQLQQRRANQRLGAEVMSQRIREMSD NDPRVVALVIRQWMSNDHE

$>$ P75955

MKQKELWINQIKGLCICLVVIYHSVITFYPHLTTFQHPLSEVLSKCWIYFNLYLAPFRMP VFFFISGYLIRRYIDSVPWGNCLDKRIWNIFWVLALWGVVQWLALSALNQWLAPERDLSN ASNAAYADSTGEFLHGMITASTSLWYLYALIVYFVVCKIFSRLALPLFALFVLLSVAVNF VPTPWWGMNSVIRNLPYYSLGAWFGATIMTCVKEVPLRRHLLMASLLTVLAVGAWLFTIS LLLSLVS IVVIMKLFYQYEQRFGMRSTSLLNVIGSNTIAIYTTHRILVEIFSLTLLAQMN AARWSPQVELTLLLVYPFVSLF I CTVAGLLVRKLSQRAFSDLLFSPPSLPAAVSYSR $>$ P 75958

AMPLSLLIGLRFSRGRRRGGMVSLISVISTIGIALGVAVLIVGLSAMNGFERELNNRILA VVPHGEIEAVDQPWTNWQEALDHVQKVPGIAAAAPYINFTGLVESGANLRAIQVKGVNPQ QEQRLSALPSFVQGDAWRNFKAGEQQ I I I GKGVADALKVKQGDWVS IM I PNSNPEHKLMQ PKRVRLHVAGILQLSGQLDHSFAMI PLADAQQYLDMGSSVSGIALKMTDVFNANKLVRDA GEVTNSYVYIKSWIGTYGYMYRDIQMIRAIMYLAMVLVIGVACFNIVSTLVMAVKDKSGD IAVLRTLGAKDGLIRA I FVWYGLLAGLFGSLCGVI I GVVVSLQLTP I IEW IEKLIGHQFL SSDIYFIDFLPSELHWLDVFYVLVTALLLSLLASWYPARRASNIDPARVLSGQ

$>$ POA1J9

MSNLSGTDKSVILLMTIGEDRAAEVFKHLSTREVQALSTAMANVRQISNKQLTDVLSEFE QEAEQFAALNINANEYLRSVLVKALGEERASSLLEDILETRDTTSGIETLNFMEPQSAAD LIRDEHPQI IATILVHLKRSQAADI LALFDERLRHDVMLR IATFGGVQPAALAELTEVLN GLLDGQNLKRSKMGGVRTAAEI INLMKTQQEEAVITAVREFDGELAQKI IDEMFLFENLV DVDDRS IQRLLQEVDSESLLIALKGAEPPLREKFLRNMSQRAADILRDDLANRGPVRLSQ VENEQKAILLIVRRLAETGEMVIGSGEDTYV

$>$ P75885

MNKGKVMKHKLSAILMAFMLTTPAAFAAPEATNGTEATTGTTGTTTTTTGATTTATTTGG VAAGAVGTATVVGVATAVGVATLAVVAANDSGDGGSHNTSTTTSTTR

$>$ POAC4 1

MKLPVREFDAVVIGAGGAGMRAALQISQSGQTCALLSKVFPTRSHTVSAQGGITVALGNT HEDNWEWHMYDTVKGSDY IGDQDAIEYMCKTGPEAILELEHMGLPFSRLDDGR IYQRPFG GQSKNFGGEQAARTAAAADRTGHALLHTLYQQNLKNHTTIFSEWYALDLVKNQDGAVVGC TALCIETGEVVYFKARATVLATGGAGRIYQSTTNAHINTGDGVGMAIRAGVPVQDMEMWQ FHPTGIAGAGVLVTEGCRGEGGYLLNKHGERFMERYAPNAKDLAGRDVVARS IMIEIREG RGCDGPWGPHAKLKLDHLGKEVLESRLPGILELSRTFAHVDPVKEP I PVIPTCHYMMGGI PTKVTGQALTVNEKGEDVVVPGLFAVGEIACVSVHGANRLGGNSLLDLVVFGRAAGLHLQ ESIAEQGALRDASESDVEASLDRLNRWNNNRNGEDPVAIRKALQECMQHNFSVFREGDAM AKGLEQLKVIRERLKNARLDDTSSEFNTQRVECLELDNLMETAYATAVSANFRTESRGAH SRFDFPDRDDENWLCHSLYLPESESMTRRSVNMEPKLRPAFPPKIRTY

$>$ POAC4 4

MVSNASALGRNGVHDF ILVRATAIVLTLYI IYMVGFFATSGELTYEVWIGFFASAFTKVF TLLALFS ILIHAW IGMWQVLTDYVKPLALRLMLQLVIVVALVVYVIYGFVVVWGV

$>$ POAD19

MMANIWWSLPLTLIVFFAARKLAARYKFPLLNPLLVAMVVI I P FLMLTGISYDSYFKGSE VLNDLLQPAVVALAYPLYEQLHQIRARWKSIITICFIGSVVAMVTGTSVALLMGASPEIA AS ILPKSVTTP IAMAVGGS I GGI PAISAVCVIFVGILGAVFGHTLLNAMR IRTKAARGLA 
MGTASHALGTARCAELDYQEGAFSSLALVLCGI ITSLIAPFLFP I I LAVMG

$>$ P06030

AHVKNHDYQILPPS IWPFFGAIGAFVMLTGAVAWMKGITFFGLPVEGPWMFLIGLVGVLY VMFGWWADVVNEGETGEHTPVVRIGLQYGF I LF IMSEVMFFVAWFWAF I KNALYPMGPDS PIKDGVWPPEGIVTFDPWHLPLINTLILLLSGVAVTWAHHAFVLEGDRKTTINGLIVAVI LGVCFTGLQAYEYSHAAFGLADTVYAGAFYMATGFHGAHVI I GTIFLFVCLIRLLKGQMT QKQHVGFEAAAWYWHFVDVVWLFLFVVIYIWGR

$>$ P75962

MSQDSKVFFRIFLGIGLVLILISVVVFYNQFTYSKDAIHTEGVIVDTVWHSSHSHRTGKD GSWYPVVAFRPTPDYTLIFNSSIGSDFYEDSEGDKVNVYYSPGHPEKAEINNPWVNFFKW GFIGIMGVIFIAVGLLISMPSSKKSRRKRK

$>$ POAFJ7

MLHLFAGLDLHTGLLLLLALAFVLFYEAINGFHDTANAVATVIYTRAMRSQLAVVMAAVF NFLGVLLGGLSVAYAIVHMLPTDLLLNMGSSHGLAMVF SMLLAAI IWNLGTWYFGLPASS SHTLIGA I IGIGLTNALMTGTSVVDALNI PKVLS I FGSLIVSP IVGLVFAGGLIFLLRRY WSGTKKRARIHLTPAEREKKDGKKKPPFWTRIALILSA IGVAF SHGANDGQKGIGLVMLV LIGVAPAGFVVNMNATGYEITRTRDAINNVEAYFEQHPALLKQATGADQLVPAPEAGATQ PAEFHCHPSNTINALNRLKGMLTTDVESYDKLSLDQRSQMRRIMLCVSDTIDKVVKMPGV SADDQRLLKKLKSDMLSTIEYAPVWI IMAVALALGIGTMIGWRRVATTIGEKIGKKGMTY AQGMSAQMTAAVS IGLASYTGMPVSTTHVLSSSVAGTMVVDGGGLQRKTVTS ILMAWVFT LPAAVLLSGGLYWLSLQFL

$>$ P2 0471

MLQNTTQSNLPREPEAKQIDYNDSIRSTYFS IDDLRACGASLAEKGTSALPGFFPFEFRA RHRENEKEILRVYRATAADVEAGAS ITPAAEWLLDNHHVVEEAIQEVRRDFPRRFYRQLP TLSVSGTVIPRTMALAWLYVAHTHSTVTRES ITAMVEGFQEHETLKIGELWALPS ILRFV LIENLRRIAIRVERSRGMRRKANEVADQLIRLNDPEGCRTLLVESEALAADNTFIAQLLY RMRDGSQSSGAVIAWIEERLERRGTDVEEALVAEQNRLSSGNATMSNI IRSLREIDDTDW AVWFESVSKIDATLREGSDYAALDFGSRNTYRDTIEKLARRSGHSEHEVTEIAIEMVEEA KAAAAVEAPLQEPNVGSFLVGKQRLALEKRIGYSPS I FQHLIRSVRKLDWFA IAGPNILL TILAMIVVYAFVS PMDI PSGAKL IMLLLFALPASEGAMGLFNTVFTLFAKPSRLVGYEFL DGI PEDARTLVVVPCLIAKRDHVDELVRNLEVHYLANPRGEIYFALLSDWADSKSEEAPA DTDVLEYAKREIASLSARYAYDGKTRFFLLHRRRLYNEAEGVWMGWERKRGKLHELNLLL RGDRDTSFLQGANMVPEGVQYVMTLDSDTRLMRDAVTKLVGKLYHP INRPVVNPRTQEVV TGYSLLQPRVTPSLTTGSEASAFQRIFTINRGIDPYVFTVSDVYQDIAGEGSFTGKGLYH VDAFEAALKSRIEENAVLSHDLLEGSYARCALVTDIELVEDFPIRYEVEMSRQHRWARGD WQLLPYI FNPKNGLSMLGRWKMYDNLRRSLI PVAWLAASVMGWYYMEPTPALIWQLVLIF SLFVAPTLSLISGIMPRRNDIVARAHLHTVLSDIRAANAQVALRIVF IAHNAAMMADAIV RSLYRTFVSRKLMLEWRTAAQVQSAGHGS IGDYFRAMWTAPALALVSLALAAISDTGLPF IGLPFALIWAASPAVAWFVSQSAETEDQLVVSEEAIEEMRKIARRTWRYFEAFVTAEQNF LPPDNFQETPQPVLAERTSPTNIGVYLLSVMSARSFGWIGFEETITRLEQTIATIDRMPK YRGHLFNWYRTRGLEPMEPRYVSSVDSGNLAGHLIAVSSMCREWAEAPSAHVQGNLDGIG DVAA ILKEALNELPDDRKTVRPLRRLVEERIAGFQNALAAVKRERELAS IRVINLAVLAR DMHKLTVNLDHEVRTVQSGEVATWAGSLVAACEAHIADGVFDLGAIEALRQRLLVLKERA RDIAFSMDFSFLFRPERRLLSIGYRVNANELDEACYDLLASEARLTSLFAIAKGDLPTEH WYKLGRP IVP IGARGALVSWSGSMFEYLMPPLVMQERQGGILNQTNNLVVQEQINHGRRL GTPWGISEAAFNARDHELTYQYTNFGVPTLGLKRGLGQNAVIAPYAS ILACMYDPKSALA NLARLREVGALGAYGYHDAVDFTPTRVPEGQKCAVVRNYYAHHHGMSVAAVANVVFNGQL REWFHADPVIEAAELLLQEKAPRDI PVMAAKREPEALGKGQADLLRPEVRVVEDPINQDR ETVLLSNGHYSVMLTATGAGYARWNGQSVTRWTPDPVEDRTGTFIFLRDTVTGDWWSATA EPRRAPGEKTVTRFGDDKAEFVKTVGDLTSEVECIVATEHDAEGRRVILLNTGTEDRFIE VTSYAEPVLAMDDADSSHPTFSKMFLRTEISRHGDVIWVSRNKRSPGDPDIEVAHLVTDN AGSERHTQAETDRRRFLGQGRTLAEAAAFDPGATLSGTDGFTLDPIVSLRRVVRVPAGKK VSVIFWTIAAPDREGVDRAIDRYRHPETFNHELIHAWTRSQVQMRHVGITSKEAASFQML GRYLVYPDMHLRADAETVKTGLASQSALWPLAISGDFP IFCLRINDDGDLGIAREALRAQ EYLRARGITADLVVVNERASSYAQDLQHTLDSMCENLRLRGLSDGPRQHIFAVRRDLMEP ETWSTLISASRAVFHARNGTISDQIARATSLYSKSSEKKEEGAEMLLPVIREADARTAVE LDGGDLDFWNGFGGFAEDGREYAVRLRGGEATPQPWINVISNEQFGFHVSAEGAAFSWSR NSRDYQLTPWTNDAVVNRPGEA I FVRDMASGAVLTPYAALSRRKSALFETRHGLGYSRFL STQDELEIEAMHTVHRTLPAKLVRLTIRNRSSAARKLRVYGYAEWVLGNNRSRTAPFVLS 
EWDESAKTLVATNPYSIDYPGRCAFFASDGDIAGYTASRREFLGRAGGILAPQAVISGAE LTGSTDVDGDACAALATDITVEAGVERQVTFFLGDADNPDQVRAVLEELRADSFGAALEA AKAFWGDFTGVVKVETPDRAFNHMINHWLPYQALGCR IMARSAFYQASGAFGFRDQLQDT LAFLIHRPALARAQILNAAARQFVEGDVQHWWLPGTDAGVRTMISDDVVWLAHAVAHYCA VTGEEDILKEKVPFITGPALEEGQHDSFYKPDVADEVGDVYEHCARALDLAIHRTGANGL PLILGGDWNDGMNRVGEAGEGTSVWLGWFLAGTLRAFLPYARARKDKPRVALWERHLEAL KDALEQAGWDGDYYRRGYYDDDTPLGSAENGECRIDS IAQSWSTLSGEGDKERSLRAMDA VMAELVDPEKRIVRLFTPPLETTKQDPGYIKAYPPGVRENGGQYTHAATWVVLAFAAQER AEEAWRTFRMLNPVSHALSQVDAEHYRVEPYVVAADIYGEGALAGRGGWTWYTGSAGWLY RAGVEGILGIRKRGDKLLIRPVLPSEWPGYSAEVRVNGTTHRISVSRDSKSGEPVVSVNN SVTKNAHEGVLL

$>$ P06974

MGDS ILSQAEIDALLNGDSEVKDEPTASVSGESDIRPYDPNTQRRVVRERLQALEI INER FARHFRMGLFNLLRRSPDITVGAIRIQPYHEFARNLPVPTNLNLIHLKPLRGTGLVVFSP SLVFIAVDNLFGGDGRFPTKVEGREFTHTEQRVINRMLKLALEGYSDAWKAINPLEVEYV RSEMQVKFTNITTSPNDIVVNTPFHVEIGNLTGEFNICLPFSMIEPLRELLVNPPLENSR NEDQNWRDNLVRQVQHSQLELVANFADISLRLSQILKLNPGDVLPIEKPDRI IAHVDGVP VLTSQYGTLNGQYALRIEHLINPILNSLNEEQPK

$>$ P23889

MARSGFEVQKVTVEALFLREIRTRFGKFRLGYLWAILEPSAHLLILLGILGYVMHRTMPD ISFPVFLLNGLI PFFIFSSISKRS IGAIEANQGLFNYRPVKPIDTI IARALLETLIYVAV YILLMLIVWMTGEYFEITNFLQLVLTWSLLI I LSCGVGLI FMVVGKTF PEMQKVLPILLK PLYFISCIMFPLHS I PKQYWSYLLWNPLVHVVELSREAVMPGYISEGVSLNYLAMFTLVT LFIGLALYRTREEAMLTS

$>$ POAD2 7

MVTHRQRYREKVSQMVSWGHWFALFNILLSLVIGSRYLFIADWPTTLAGRIYSYVSI IGH FSFLVFATYLLILFPLTFIVGSQRLMRFLSVILATAGMTLLLIDSEVFTRFHLHLNPIVW QLVINPDENEMARDWQLMF ISVPVILLLELVFATWSWQKLRSLTRRRRFARPLAAFLFIA FIASHVVYIWADANFYRPITMQRANLPLSYPMTARRFLEKHGLLDAQEYQRRLIEQGNPD AVSVQYPLSELRYRDMGTGQNVLLITVDGLNYSRFEKQMPALAGFAEQNISFTRHMSSGN TTDNGIFGLFYGISPSYMDGILSTRTPAALITALNQQGYQLGLFSSDGFTSPLYRQALLS DFSMPSVRTQSDEQTATQWINWLGRYAQEDNRWFSWVSFNGTNIDDSNQQAFARKYSRAA GNVDDQINRVLNALRDSGKLDNTVVI ITAGRGIPLSEEEETFDWSHGHLQVPLVIHWPGT PAQR INALTDHTDLMTTLMQRLLHVSTPASEYSQGQDLFNPQRRHYWVTAADNDTLAITT PKKTLVLNNNGKYRTYNLRGERVKDEKPQLSLLLQVLTDEKRFIAN $>$ POABV6

MARARGRGRRDLKSEINIVPLLDVLLVLLLI FMATAP I ITQSVEVDLPDATESQAVSSND NPPVIVEVSGIGQYTVVVEKDRLERLPPEQVVAEVSSRFKANPKTVFLIGGAKDVPYDEI IKALNLLHSAGVKSVGLMTQPI

$>$ P2 7303

MSANAETQTPQQPVKKSGKRKRLLLLLTLLFI I IAVAIGIYWFLVLRHFEETDDAYVAGN QIQIMSQVSGSVTKVWADNTDFVKEGDVLVTLDPTDARQAFEKAKTALASSVRQTHQLMI NSKQLQANIEVQKIALAKAQSDYNRRVPLGNANLIGREELQHARDAVTSAQAQLDVAIQQ YNANQAM I LGTKLEDQPAVQQAATEVRNAWLALERTR I ISPMTGYVSRRAVQPGAQISPT TPLMAVVPATNMWVDANFKETQIANMRIGQPVTITTDIYGDDVKYTGKVVGLDMGTGSAF SLLPAQNATGNWIKVVQRLPVRIELDQKQLEQYPLRIGLSTLVSVNTTNRDGQVLANKVR STPVAVSTAREISLAPVNKLIDDIVKANAG

$>$ P39398

MEKENITIDPRSSFTPSSSADI PVPPDGLVQRSTRIKRIQTTAMLLLFFAAVINYLDRSS LSVANLTIREELGLSATEIGALLSVFSLAYGIAQLPCGPLLDRKGPRLMLGLGMFFWSLF QAMSGMVHNFTQFVLVRIGMGIGEAPMNPCGVKVINDWFNIKERGRPMGFFNAASTIGVA VSPP ILAAMMLVMGWRGMF ITIGVLGIFLAIGWYMLYRNREHVELTAVEQAYLNAGSVNA RRDPLSFAEWRSLFRNRTMWGMMLGFSGINYTAWLYLAWLPGYLQTAYNLDLKSTGLMAA I PFLFGAAGMLVNGYVTDWLVKGGMAP I KSRKICI IAGMFCSAAFTLIVPQATTSMTAVL LIGMALFCIHFAGTSCWGLIHVAVASRMTASVGS IQNFASF I CASFAP I ITGF IVDTTHS FRLALI I CGCVTAAGALAYIFLVRQPINDP

$>Q 07282$

MNRTVMMALVI I FLDAMGIGI IMPVLPALLREFVGKANVAENYGVLLALYAMMQVIFAPL LGRWSDR IGRRPVLLLSLLGATLDYALMATASVVWVLYLGRLIAGITGATGAVAASTIAD 
VTPEESRTHWFGMMGACFGGGMIAGPVIGGFAGQLSVQAPFMFAAAINGLAFLVSLFILH ETHNANQVSDELKNETINETTSS IREMIS PLSGLLVVFF I IQLIGQI PATLWVLFGEERF AWDGVMVGVSLAVFGLTHALFQGLAAGFIAKHLGERKAIAVGILADGCGLFLLAVITQSW MVWPVLLLLACGGITLPALQGI ISVRVGQVAQGQLQGVLTSLTHLTAVIGPLVFAFLYSA TRETWNGWVWI IGCGLYVVALI I LRFFHPGRVIHP INKSDVQQRI

$>$ POAGH1

MRHLRNI FNLGI KELRSLLGDKAMLTLIVFSFTVSVYSSATVTPGSLNLAPIAIADMDQS QLSNRIVNSFYRPWFLPPEMITADEMDAGLDAGRYTFAINI PPNFQRDVLAGRQPDIQVN VDATRMSQAFTGNGYIQNI INGEVNSFVARYRDNSEPLVSLETRMRFNPNLDPAWFGGVM AI INNITMLA IVLTGSAL IREREHGTVEHLLVMP ITPFEIMMAKIWSMGLVVLVVSGLSL VLMVKGVLGVP IEGS I PLFMLGVALSLFATTS IGI FMGTIARSMPQLGLLVILVLLPLQM LSGGSTPRESMPQMVQDIMLTMPTTHFVSLAQAILYRGAGFEIVWPQFLTLMAIGGAFFT IALLRFRKTIGTMA

$>$ POAGH5

MPYDSVYSEKRPPGTLRTAWRKFYSDASAMVGLYGCAGLAVLCIFGGWFAPYGIDQQFLG YQLLPPSWSRYGEVSFFLGTDDLGRDVLSRLLSGAAPTVGGAFVVTLAATICGLVLGTFA GATHGLRSAVLNH ILDTLLA I PSLLLA I IVVAFAGPSLSHAMFAVWLALLPRMVRS IYSM VHDELEKEYVIAARLDGASTLNILWFAVMPNITAGLVTEITRALSMAILDIAALGFLDLG AQLPSPEWGAMLGDALELIYVAPWTVMLPGAAIMI SVLLVNLLGDGVRRAI IAGVE $>$ POAFK2

MDFFSVQNILVHIPIGAGGYDLSWIEAVGTIAGLLCIGLASLEKISNYFFGLINVTLFGI IFFQIQLYASLLLQVFFFAANIYGWYAWSRQTSQNEAELKIRWLPLPKALSWLAVCVVSI GLMTVFINPVFAFLTRVAVMIMQALGLQVVMPELQPDAFPFWDSCMMVLSIVAMILMTRK YVENWLLWVI INVISVVIFALQGVYAMSLEYI ILTF IALNGSRMWINSARERGSRALSH $>$ P31119 MLFSFFRNLCRVLYRVRVTGDTQALKGERVLITPNHVSFIDGILLGLFLPVRPVFAVYTS ISQQWYMRWLKSF IDFVPLDPTQPMA I KHLVRLVEQGRPVVIFPEGRITTTGSLMKIYDG AGFVAAKSGATVIPVRIEGAELTHFSRLKGLVKRRLFPQITLHILPPTQVAMPDAPRARD RRKIAGEMLHQIMMEARMAVRPRETLYESLLSAMYRFGAGKKCVEDVNFTPDSYRKLLTK TLFVGRILEKYSVEGERIGLMLPNAGISAAVIFGAIARRRMPAMMNYTAGVKGLTSAITA AEIKTIFTSRQFLDKGKLWHLPEQLTQVRWVYLEDLKADVTTADKVWIFAHLLMPRLAQV KQQPEEEALILFTSGSEGHPKGVVHSHKS I LANVEQI KTIADFTTNDRFMSALPLFHSFG LTVGLFTPLLTGAEVFLYPSPLHYRIVPELVYDRSCTVLFGTSTFLGHYARFANPYDFYR LRYVVAGAEKLQESTKQLWQDKFGLRILEGYGVTECAPVVS INVPMAAKPGTVGRILPGM DARLLSVPGIEEGGRLQLKGPNIMNGYLRVEKPGVLEVPTAENVRGEMERGWYDTGDIVR FDEQGFVQIQGRAKRFAKIAGEMVSLEMVEQLALGVSPDKVHATAI KSDASKGEALVLFT TDNELTRDKLQQYAREHGVPELAVPRDIRYLKQMPLLGSGKPDFVTLKSWVDEAEQHDE $>$ POAGH8

MAATKPAFNPPGKKGDI IFSVLVKLAALIVLLMLGGI IVSLIISSWPSIQKFGLAFLWTK EWDAPNDI YGALVP IYGTLVTSF IALLIAVPVSFGIALFLTELAPGWLKRPLGIAIELLA A I PS IVYGMWGLF I FAPLFAVYFQEPVGN IMSNI P IVGALFSGPAFGIGILAAGVILAIM I I PYIAAVMRDVFEQTPVMMKESAYGIGCTTWEVIWR I VLPFTKNGVIGGIMLGLGRALG ETMAVTF I IGNTYQLDSASLYMPGNS ITSALANEFAEAESGLHVAALMELGLILFVITF I VLAASKFMIMRLAKNEGAR

$>$ P23894

MMRIALFLLTNLAVMVVFGLVLSLTGIQSSSVQGLMIMALLFGFGGSFVSLLMSKWMALR SVGGEVIEQPRNERERWLVNTVATQARQAGIAMPQVAIYHAPDINAFATGARRDASLVAV STGLLQNMS PDEAEAVIAHEISHIANGDMVTMTLIQGVVNTFVIFISRILAQLAAGFMGG NRDEGEESNGNPLIYFAVATVLELVFGILASI ITMWFSRHREFHADAGSAKLVGREKMIA ALQRLKTSYEPQEATSMMALCINGKSKSLSELFMTHPPLDKRIEALRTGEYLK $>$ POAEN4 MISRVTEALSKVKGSMGSHERHALPGVIGDDLLRFGKLPLCLFICI ILTAVTVVTTAHHT RLLTAQREQLVLERDALDIEWRNLILEENALGDHSRVERIATEKLQMQHVDPSQENIVVQ $\mathrm{K}$

$>$ P15877

MAINNTGSRRLLVTLTALFAALCGLYLLIGGGWLVAIGGSWYYPIAGLVMLGVAWMLWRS KRAALWLYAALLLGTMIWGVWEVGFDFWALTPRSDILVFFGIWLILPFVWRRLVI PASGA VAALVVALLISGGILTWAGFNDPQEINGTLSADATPAEAIS PVADQDWPAYGRNQEGQRF SPLKQINADNVHNLKEAWVFRTGDVKQPNDPGEITNEVTPI KVGDTLYLCTAHQRLFALD 
AASGKEKWHYDPELKTNESFQHVTCRGVSYHEAKAETASPEVMADCPRRI ILPVNDGRLI AINAENGKLCETFANKGVLNLQSNMPDTKPGLYEPTSPP I ITDKT IVMAGSVTDNFSTRE TSGVIRGFDVNTGELLWAFDPGAKDPNAI PSDEHTFTFNSPNSWAPAAYDAKLDLVYLPM GVTTPDIWGGNRTPEQERYASS I LALNATTGKLAWSYQTVHHDLWDMDLPAQPTLADITV NGQKVPVIYAPAKTGNIFVLDRRNGELVVPAPEKPVPQGAAKGDYVTPTQPFSELSFRPT KDLSGADMWGATMFDQLVCRVMFHQMRYEGIFTPPSEQGTLVFPGNLGMFEWGGISVDPN REVAIANPMALPFVSKLIPRGPGNPMEQPKDAKGTGTESGIQPQYGVPYGVTLNPFLSPF GLPCKQPAWGYISALDLKTNEVVWKKRIGTPQDSMPFPMPVPVPFNMGMPMLGGPISTAG NVLFIAATADNYLRAYNMSNGEKLWQGRLPAGGQATPMTYEVNGKQYVVISAGGHGSFGT KMGDYIVAYALPDDVK

$>$ POAE06

MNKNRGFTPLAVVLMLSGSLALTGCDDKQAQQGGQQMPAVGVVTVKTEPLQITTELPGRT SAYRIAEVRPQVSGI ILKRNFKEGSDIEAGVSLYQIDPATYQATYDSAKGDLAKAQAAAN IAQLTVNRYQKLLGTQYISKQEYDQALADAQQANAAVTAAKAAVETARINLAYTKVTSPI SGRIGKSNVTEGALVQNGQATALATVQQLDPIYVDVTQSSNDFLRLKQELANGTLKQENG KAKVSLITSDGIKFPQDGTLEFSDVTVDQTTGS ITLRAI FPNPDHTLLPGMFVRARLEEG LNPNAILVPQQGVTRTPRGDATVLVVGADDKVETRPIVASQAIGDKWLVTEGLKAGDRVV ISGLQKVRPGVQVKAQEVTADNNQQAASGAQPEQSKS

$>$ P80586

MTNGKIWLVVKPTVGLPIGMLFAALLAVLIHGLLFVDGRLKSWWSEFPVAKPAVVSVQAA PAPVAAEVK

$>$ POAAZO

MEDETLGFFKKTSSSHARLNVPALVQVAALAI IMIRGLDVLMI FNTLGVRGIGEF IHRSV QTWSLTLVFLSSLVLVFIEIWCAFSLVKGRRWARWLYLLTQITAASYLWAASLGYGYPEL FSIPGESKREIFHSLMLQKLPDMLILMLLFVPSTSRRFFQLQ

$>$ P02919

MAGNDREPIGRKGKPTRPVKQKVSRRRYEDDDDYDDYDDYEDEEPMPRKGKGKGKGRKPR GKRGWLWLLLKLAIVFAVLIAIYGVYLDQKIRSR IDGKVWQLPAAVYGRMVNLEPDMTIS KNEMVKLLEATQYRQVSKMTRPGEFTVQANS I EMIRRPFDFPDSKEGQVRARLTFDGDHL ATIVNMENNRQFGFFRLDPRLITMISSPNGEQRLFVPRSGFPDLLVDTLLATEDRHFYEH DGISLYSIGRAVLANLTAGRTVQGASTLTQQLVKNLFLSSERSYWRKANEAYMALIMDAR YSKDRILELYMNEVYLGQSGDNEIRGFPLASLYYFGRPVEELSLDQQALLVGMVKGAS IY NPWRNPKLALERRNLVLRLLQQQQ I IDQELYDMLSARPLGVQPRGGVISPQPAFMQLVRQ ELQAKLGDKVKDLSGVKI FTTFDSVAQDAAEKAAVEGI PALKKQRKLSDLETA IVVVDRF SGEVRAMVGGSEPQFAGYNRAMQARRS IGSLAKPATYLTALSQPKIYRLNTWIADAPIAL RQPNGQVWSPQNDDRRYSESGRVMLVDALTRSMNVPTVNLGMALGLPAVTETWIKLGVPK DQLHPVPAMLLGALNLTPIEVAQAFQTIASGGNRAPLSALRSVIAEDGKVLYQSFPQAER AVPAQAAYLTLWTMQQVVQRGTGRQLGAKYPNLHLAGKTGTTNNNVDTWFAGIDGSTVTI TWVGRDNNQPTKLYGASGAMS IYQRYLANQTPTPLNLVPPEDIADMGVDYDGNFVCSGGM RILPVWTSDPQSLCQQSEMQQQPSGNPFDQSSQPQQQPQQQPAQQEQKDSDGVAGWIKDM FGSN

$>$ P11667

MFSYYFQGLALGAAMILPLGPQNAFVMNQGIRRQYHIMIALLCAISDLVLICAGIFGGSA LLMQSPWLLALVTWGGVAFLLWYGFGAFKTAMSSNIELASAEVMKQGRWKI IATMLAVTW LNPHVYLDTFVVLGSLGGQLDVEPKRWFALGTISASFLWFFGLALLAAWLAPRLRTAKAQ RI INLVVGCVMWF IALQLARDGIAHAQALFS

$>$ P25714

MDSQRNLLVIALLFVSFMIWQAWEQDKNPQPQAQQTTQTTTTAAGSAADQGVPASGQGKL ISVKTDVLDLTINTRGGDVEQALLPAYPKELNSTQPFQLLETS PQF IYQAQSGLTGRDGP DNPANGPRPLYNVEKDAYVLAEGQNELQVPMTYTDAAGNTFTKTFVLKRGDYAVNVNYNV QNAGEKPLEISSFGQLKQSITLPPHLDTGSSNFALHTFRGAAYSTPDEKYEKYKFDTIAD NENLNI SSKGGWVAMLQQYFATAWI PHNDGTNNFYTANLGNGIAAIGYKSQPVLVQPGQT GAMNSTLWVGPEIQDKMAAVAPHLDLTVDYGWLWF ISQPLFKLLKWI HSFVGNWGFS I I I ITFIVRGIMYPLTKAQYTSMAKMRMLQPKIQAMRERLGDDKQRISQEMMALYKAEKVNPL GGCFPLLIQMP I FLALYYMLMGSVELRQAPFALW I HDLSAQDPYYILPILMGVTMFF IQK MSPTTVTDPMQQKIMTFMPVIFTVFFLWFPSGLVLYYIVSNLVTI IQQQLIYRGLEKRGL HSREKKKS

$>$ P42628

MEIASNKGVIADASTPAGRAGMSESEWREAI KFDSTDTGWVIMS IGMAIGAGIVFLPVQV 
GLMGLWVFLLSSVIGYPAMYLFQRLFINTLAESPECKDYPSVISGYLGKNWGILLGALYF VMLVIWMFVYSTAITNDSASYLHTFGVTEGLLSDSPFYGLVLICILVAISSRGEKLLFKI STGMVLTKLLVVAALGVSMVGMWHLYNVGSLPPLGLLVKNAI ITLPFTLTS ILFIQTLSP MVISYRSREKSIEVARHKALRAMNIAFGILFVTVFFYAVSFTLAMGHDEAVKAYEQNISA LAIAAQF ISGDGAAWVKVVSVILNIFAVMTAFFGVYLGFREATQGIVMNILRRKMPAEKI NENLVQRGIMIFA I LLAWSA IVLNAPVLSFTS I CSP I FGMVGCLI PAWLVYKVPALHKYK GMSLYLIIVTGLLLCVSPFLAFS

$>$ P31122

MTTNTVSRKVAWLRVVTLAVAAFIFNTTEFVPVGLLSDIAQSFHMQTAQVGIMLTIYAWV VALMSLPFMLMTSQVERRKLLICLFVVFIASHVLSFLSWSFTVLVISRIGVAFAHAIFWS ITASLAIRMAPAGKRAQALSLIATGTALAMVLGLPLGRIVGQYFGWRMTFFAIGIGALIT LLCLIKLLPLLPSEHSGSLKSLPLLFRRPALMS IYLLTVVVVTAHYTAYSYIEPFVQNIA GFSANFATALLLLLGGAGI IGSVIFGKLGNQYASALVSTAIALLLVCLALLLPAANSEIH LGVLS IFWGIAMMI I GLGMQVKVLALAPDATDVAMALFSGIFNIGIGAGALVGNQVSLHW SMSMIGYVGAVPAFAALIWS I I IFRRPVTLEEQTQ

$>$ P 77726

MNDYKMTPGERRATWGLGTVFSLRMLGMFMVLPVLTTYGMALQGASEALIGIAIGIYGLT QAVFQI PFGLLSDRIGRKPLIVGGLAVFAAGSVIAALSDS IWGI ILGRALQGSGAIAAAV MALLSDLTREQNRTKAMAF I GVSFGITFA IAMVLGP I ITHKLGLHALFWMIAILATTGIA LTIWVVPNSSTHVLNRESGMVKGSFSKVLAEPRLLKLNFGIMCLHILLMSTFVALPGQLA DAGFPAAEHWKVYLATMLIAFGSVVPFI IYAEVKRKMKQVFVFCVGLIVVAEIVLWNAQT QFWQLVVGVQLFFVAFNLMEALLPSLISKESPAGYKGTAMGVYSTSQFLGVAIGGSLGGW INGMFDGQGVFLAGAMLAAVWLTVASTMKEPPYVSSLRIEI PANIAANEALKVRLLETEG IKEVLIAEEEHSAYVKIDSKVTNRFEIEQA

$>$ P31125

MSRKDGVLALLVVVVWGLNFVVIKVGLHNMPPLMLAGLRFMLVAFPAIFFVARPKVPLNL LLGYGLTISFAQFAFLFCA INFGMPAGLASLVLQAQAFFTIMLGAFTFGERLHGKQLAGI ALAIFGVLVLIEDSLNGQHVAMLGFMLTLAAAFSWACGNIFNKKIMSHSTRPAVMSLVIW SALIPI IPFFVASLILDGSATMIHSLVTIDMTTILSLMYLAFVATIVGYGIWGTLLGRYE TWRVAPLSLLVPVVGLASAALLLDERLTGLQFLGAVLIMTGLYINVFGLRWRKAVKVGS $>$ P21503

MSTYTQPVMLLLSGLLLLTLAIAVLNTLVPLWLAQEHMSTWQVGVVSSSYFTGNLVGTLL TGYVIKRIGFNRSYYLASF I FAAGCAGLGLMIGFWSWLAWRFVAGVGCAMIWVVVESALM CSGTSRNRGRLLAAYMMVYYVGTFLGQLLVSKVSTELMSVLPWVTGLTLAGILPLLFTRV LNQQAENHDSTS I TSMLKLRQARLGVNGC I I SGIVLGSLYGLMPLYLNHKGVSNAS IGFW MAVLVSAGILGQWPIGRLADKFGRLLVLRVQVFVVILGS IAMLSQAAMAPALF ILGAAGF TLYPVAMAWACEKVEHHQLVAMNQALLLSYTVGSLLGPSFTAMLMQNFSDNLLFIMIASV SFIYLLMLLRNAGHTPKPVAHV

$>$ POAFL1

MNNLPVVRSPWRIVILLLGFTFLYAPMLMLVIYSFNSSKLVTVWAGWSTRWYGELLRDDA MMSAVGLSLTIAACAATAAAILGTIAAVVLVRFGRFRGSNGFAFMITAPLVMPDVITGLS LLLLFVALAHAIGWPADRGMLTIWLAHVTFCTAYVAVVISSRLRELDRS IEEAAMDLGAT PLKVFFVITLPMIMPAI ISGWLLAFTLSLDDLVIASFVSGPGATTLPMLVFSSVRMGVNP EINALATLILGAVGIVGFIAWYLMARAEKQRIRDIQRARRG

$>$ P16683

MPDAVQAPYPAYRPEPNMQTITIAPPKRSWFSLLSWAVVLAVLVVSWQGAEMAPLTLIKD GGNMATFAADF FP PDF SQWQDYLTEMAVTLQIAVWGTALAVVLS I PFGLMSAENLVPWWV YQPVRRLMDACRAINEMVFAMLFVVAVGLGPFAGVLALFIHTTGVLSKLLSEAVEAIEPG PVEGIRATGANKLEEILYGVLPQVMPLLISYSLYRFESNVRSATVVGMVGAGGIGVTLWE AIRGFQFQQTCALMVLI IVTVSLLDFLSQRLRKHFI

(6) 1 nucleoid protein

$>$ Q6LPL4

MNMEPRYKPMSS INIEEYMPEKLAKAIANPLFPALDNALRSGRHVSSEDLDNHALLIEFE RELGMFYRRYNTELIRAPEGFFYLRPRSTSLIGRSVLSEIDMLVGKVLCFLYLSPERLAH EGIFTNQELFDELLVLADEKKLMKFVTHRASGSDLDREKLYDKVKTSLRRLRRIGMLIPI GENGKFRISESVFRFGADVRTGDDVREAQLRLIRDGEAVVHQQEPSQSSLLDGFDADDTG HHDSELTMQE

(7) 13 outer membrane proteins >POA927 
MKKTLLAAGAVLALSSSFTVNAAENDKPQYLSDWWHQSVNVVGSYHTRFGPQIRNDTYLE YEAFAKKDWFDFYGYADAPVFFGGNSDAKGIWNHGSPLFMEIEPRFS IDKLTNTDLSFGP FKEWYFANNYIYDMGRNKDGRQSTWYMGLGTDIDTGLPMSLSMNVYAKYQWQNYGAANEN EWDGYRFKIKYFVPITDLWGGQLSYIGFTNFDWGSDLGDDSGNAINGIKTRTNNSIASSH ILALNYDHWHYSVVARYWHDGGQWNDDAELNFGNGNFNVRSTGWGGYLVVGYNF

$>$ P23988

MKNI ILSTLVITTSVLVVNVAQADTNAFSVGYAQSKVQDFKNIRGVNVKYRYEDDSPVSF ISSLSYLYGDRQASGSVEPEGIHYHDKFEVKYGSLMVGPAYRLSDNFSLYALAGVGTVKA TFKEHSTQDGDSFSNKISSRKTGFAWGAGVQMNPLENIVVDVGYEGSNISSTKINGFNVG VGYRF

$>$ P12375

MTVLNRLHVCSLLAVSSLGMLPVGVFAAEAAMPGVDHSQMQGMDHSKMQGMDHSQMQGMD HS KMQGMDHSQMQGMDSDMTTMAPSKPAAPTQSRTPIAPVTDANRAAVYRSAKGHTVHDE AANYFLLFDQLEWQDADNGSVLNWDVNGWVGGDIDRLWIRSEGERTNGKTESAELQALWG HAISPWWDLVGGVRQDFKPGSPQTWAAFGLQGLALYNFEAEATAFLGEGGQTGLRLEGDY DILLTNRLILQPTAEVNFYGQSDPQRGIGSGLSETEVGVRLRYEIRREFAPYIGVTWNRS YGNTADFAREEGEDRSEARLVLGVRMWF

$>$ P02930

MQMKKLLPILIGLSLSGFSSLSQAENLMQVYQQARLSNPELRKSAADRDAAFEKINEARS PLLPQLGLGADYTYSNGYRDANGINSNATSASLQLTQS I FDMSKWRALTLQEKAAGIQDV TYQTDQQTLILNTATAYFNVLNAIDVLSYTQAQKEAIYRQLDQTTQRFNVGLVAITDVQN ARAQYDTVLANELTARNNLDNAVEQLRQITGNYYPELAALNVENFKTDKPQPVNALLKEA EKRNLSLLQARLSQDLAREQIRQAQDGHLPTLDLTASTGISDTSYSGSKTRGAAGTQYDD SNMGQNKVGLSFSLPIYQGGMVNSQVKQAQYNFVGASEQLESAHRSVVQTVRSSFNNINA SISS INAYKQAVVSAQSSLDAMEAGYSVGTRTIVDVLDATTTLYNAKQELANARYNYLIN QLNI KSALGTLNEQDLLALNNALSKPVSTNPENVAPQTPEQNAIADGYAPDSPAPVVQQT SARTTTSNGHNPFRN

$>$ Q05811

MNIRMVLLASAAAFAASTPVLAADA IVAAEPEPVEYVRVCDAYGTGYFYIPGTETCLKIE GYIRFQVNVGDNPGGDNDSDWDAVTAVRFSSRKSDTEYGPLTGVIVMQFNADNASDQDAI LDSAYLDVAGFRAGLFYSWWDDGLSGETDDIGSVVTLHNS IRYQYESGTFYAGLSVDELE DGVYQGTFTPGVIPGTTDFTADDGPNNVGVAFGIGGTAGAFSYQVTGGWDVDNEDGAIRA MGTVEIGPGTFGLAGVYSSGPNSYYSSAEWAVAAEYAIKATDKLKITPGRWHGHVPEDFD GLGDAWKVGLTVDYQIVENFYAKASVQYLDPQDGEDSTSGYFACSVRSNHLVDAPGLRIG STTISF

$>$ P31106

MKMRLVAAAAMGLAMSTTIAATATTDATTSAPGTSLTTDTEKLSYS IGADLGKNFKKQGI EISPAAMAKGLQDGMSGGQLLLTDDQMKDVLNKFQKDLMMKRSAEFNKKAEENKSKGEAF LNENKSKEGVVSLPSGLQYNILERGDGAKPTKDDVVTVEYTGKLIDGQVFDSTEKTGKPA TFKVSQVI PGWTEALQLMPAGSTWEVYI PSNLAYGPRSVGGPIGPNETLIFKIHLISVKK SDA

$>$ P16239

MKEDKVLILRTCANNMADHGGI IWPLSGIVECKYWKPVKGFENGLTGLIWGKGSDSPLSL HADARWVVAEVDADECIAIETHGWI KF PRAEVLHVGTKTSAMQF I LHHRADYVACTEMQA GPGSPDVTSEVKVGNRSLPVTDDIDATIESGSTQPTQTIEIATYGSTLSGTHQSQLIAGY GSTETAGDSSTLIAGYGSTGTAGADSTLVAGYGSTQTAGEESSQMAGYGSTQTGMKGSDL TAGYGSTGTAGDDSSLIAGYGSTQTAGEDSSLTAGYGSTQTAQKGSDLTAGYGSTGTAGA DSSLIAGYGSTQTAGEESTQTAGYGSTQTAQKGSDLTAGYGSTGTAGDDSSLIAGYGSTQ TAGEDSSLTAGYGSTQTAQKGSDLTAGYGSTGTAGADSSLIAGYGSTQTAGEESTQTAGY GSTQTAQKGSDLTAGYGSTGTAGDDSSLIAGYGSTQTAGEDSSLTAGYGSTQTAQKGSDL TAGYGSTSTAGYESSLIAGYGSTQTAGYGSTLTAGYGSTQTAQNESDLITGYGSTSTAGA NSSLIAGYGSTQTASYNSVLTAGYGSTQTAREGSDLTAGYGSTGTAGSDSSI IAGYGSTQ TASYHSSLTAGYGSTQTAREQSVLTTGYGSTSTAGADSSLIAGYGSTQTAGYNS ILTAGY GSTQTAQEGSDLTAGYGSTSTAGADSSLIAGYGSTQTAGYNSILTAGYGSTQTAQEGSDL TSGYGSTSTAGADSSLIAGYGSTQTASYHSSLTAGYGSTQTAREQSVLTTGYGSTSTAGA DSSLIAGYGSTQTAGYHSILTAGYGSTQTAQERSDLTTGYGSTSTAGADSSLIAGYGSTQ TAGYNS ILTAGYGSTQTAQENSDLTTGYGSTSTAGYDSSLIAGYGSTQTAGYNS ILTAGY GSTQTAQENSDLTTGYGSTSTAGYESSLIAGYGSTQTASFKSTLMAGYGSSQTAREQSSL TAGYGSTSMAGYDSSLIAGYGSTQTAGYQSTLTAGYGSTQTAEHSSTLTAGYGSTATAGA 
DSSLIAGYGSSLTSGIRSFLTAGYGSTLISGLRSVLTAGYGSSLISGRRSSLTAGYGSNQ IASHRSSLIAGPESTQITGNRSMLIAGKGSSQTAGYRSTLISGADSVQMAGERGKLIAGA DSTQTAGDRSKLLAGNNSYLTAGDRSKLTAGNDCILMAGDRSKLTAGINS ILTAGCRSKL IGSNGSTLTAGENSVLIFRCWDGKRYTNVVAKTGKGGIEADMPYQMDEDNNIVNKPEE $>$ P0A3U4 MKSVILAS IAAMFATSAMAADVVVSEPSAPTAAPVDTFSWTGGYIGINAGYAGGKFKHPF SSFDKEDNEQVSGSLDVTAGGFVGGVQAGYNWQLDNGVVLGAETDFQGSSVTGSISAGAS GLEGKAETKVEWFGTVRARLGYTATERLMVYGTGGLAYGKVKSAFNLGDDASALHTWSDK TKAGWTLGAGAEYAINNNWTLKSEYLYTDLGKRNLVDVDNSFLESKVNFHTVRVGLNYKF $>$ P10641

MKKFNQSLLATAMLLAAGGANAAAFQLAEVSTSGLGRAYAGEAAIADNASVVATNPALMS LFKTAQFSTGGVYIDSRINMNGDVTSYAQI ITNQIGMKA I KDGSASQRNVVPGAFVPNLY FVAPVNDKFALGAGMNVNFGLKSEYDDSYDAGVFGGKTDLSA INLNLSGAYRVTEGLSLG LGVNAVYAKAQVERNAGLIADSVKDNQITSALSTQQEPFRDLKKYLPSKDKSVVSLQDRA AWGFGWNAGVMYQFNEANRIGLAYHSKVDIDFADRTATSLEANVI KEGKKGNLTFTLPDY LELSGFHQLTDKLAVHYSYKYTHWSRLTKLHASFEDGKKAFDKELQYSNNSRVALGASYN LYEKLTLRAGIAYDQAASRHHRSAA I PDTDRTWYSLGATYKFTPNLSVDLGYAYLKGKKV HFKEVKTIGDKRTLTLNTTANYTSQAHANLYGLNLNYSF

$>$ P31554

MKKRI PTLLATMIATALYSQQGLAADLASQCMLGVPSYDRPLVQGDTNDLPVTINADHAK GDYPDDAVFTGSVDIMQGNSRLQADEVQLHQKEAPGQPEPVRTVDALGNVHYDDNQVILK GPKGWANLNTKDTNVWEGDYQMVGRQGRGKADLMKQRGENRYTILDNGSFTSCLPGSDTW SVVGSEI I HDREEQVAE I WNARFKVGPVP I FYSPYLQLPVGDKRRSGFLI PNAKYTTTNY FEFYLPYYWNIAPNMDATITPHYMHRRGNIMWENEFRYLSQAGAGLMELDYLPSDKVYED EHPNDDSSRRWLFYWNHSGVMDQVWRFNVDYTKVSDPSYFNDFDNKYGSSTDGYATQKFS VGYAVQNFNATVSTKQFQVFSEQNTSSYSAEPQLDVNYYQNDVGPFDTRIYGQAVHFVNT RDDMPEATRVHLEPTINLPLSNNWGS INTEAKLLATHYQQTNLDWYNSRNTTKLDESVNR VMPQFKVDGKMVFERDMEMLAPGYTQTLEPRAQYLYVPYRDQSDIYNYDSSLLQSDYSGL FRDRTYGGLDRIASANQVTTGVTSRIYDDAAVERFNISVGQIYYFTESRTGDDNITWEND DKTGSLVWAGDTYWRISERWGLRGGIQYDTRLDNVATSNSS IEYRRDEDRLVQLNYRYAS PEYIQATLPKYYSTAEQYKNGISQVGAVASWPIADRWS IVGAYYYDTNANKQADSMLGVQ YSSCCYAIRVGYERKLNGWDNDKQHAVYDNAI GFNIELRGLSSNYGLGTQEMLRSNILPY QNTL

$>$ P06626

MYPMDRIQQKHARQIDLLENLTAVIQDYPNPACIRDETGKFIFCNTLFHESFLTQDQSAE KWLLSQRDFCELISVTEMEAYRNEHTHLNLVEDVF IQNRFWTISVQSFLNGHRNI I LWQF YDAAHVRHKD SYNQKTIVSDDIRNI IRRMSDDSSVSSYVNDVFYLYSTGISHNAIARILN IS ISTSKKHASLICDYFSVSNKDELI I LLYNKKF I YYLYEKAMCI INTR

$>$ P10325

MKKTNMALALLVAFSVTGCANTDIFSGDVYSASQAKEARS ITYGTIVSVRPVKIQADNQG VVGTLGGGALGGIAGSTIGGGRGQA IAAVVGAIGGAIAGSKIEEKMSQVNGAELVI KKDD GQEIVVVQKADSSFCSLVAEFVFVGGGSSLNVSVL

$>$ P16465

MKKKVVLLTLLSCFSTSGLSANETGNLGS ISESRRALQDSQREINQLIEQNRYQQLQEKA VNISPTPTLITESEHCLPIKGVYIQGITLLTEKDLNSLSPLPDQCIKSADINRLVKELTQ RYLQHGYITARIQFLRPNQHGELGLYAIEGFVERIEGGDRGVNTTLLFPRIKGQPLKLAT LDQGLDQANRLQSNKVTVDILPGTELGGSVI KLSNQRKS PWHLNIASDNYGQKNSGRWLI RTNASLDSPLGLSDFVSLNANITTDNPNTRFNRAYTLLYS I PYGGFTFSSFGSYSEYQFH QKLQTRTVNLYGDTTQVGIRGDYAFSRSQKQIDTLNIQVTHKRIRNYFSQIRLDLSSPKL TTIELGINHLQ I I PNGVLSTNLSVEKAVGWFGAEETPY IANGNGNDYRFTKVKLFTNWYQ RFSLWHSTFLFNSTFLGQYSHDTLPGVEWLSLTDKNAIRGFDQSTLSGDNGGYLRNTLSY PYRLNHFS ITPRIGVDIGQVKQHGNYKGWQGGYGLSSGLNIQYQQAQLDLEVAKGELLYH QTNSNKTKDPTQLLVKFSYLF

(8) 49 periplasm proteins

$>$ P39265

MNKYLKYFSGTLVGLMLSTSAFAAAEYAVVLKTLSNPFWVDMKKGIEDEAKTLGVSVDIF ASPSEGDFQSQLQLFEDLSNKNYKGIAFAPLSSVNLVMPVARAWKKGIYLVNLDEKIDMD NLKKAGGNVEAFVTTDNVAVGAKGASF I IDKLGAEGGEVA I I EGKAGNASGEARRNGATE AFKKASQI KLVASQPADWDRI KALDVATNVLQRNPNI KAIYCANDTMAMGVAQAVANAGK 
TGKVLVVGTDGI PEARKMVEAGQMTATVAQNPADIGATGLKLMVDAEKSGKVI PLDKAPE FKLVDSILVTQ

$>$ P02924

MHKFTKALAAIGLAAVMSQSAMAENLKLGFLVKQPEEPWFQTEWKFADKAGKDLGFEVIK IAVPDGEKTLNAIDSLAASGAKGFVICTPDPKLGSAIVAKARGYDMKVIAVDDQFVNAKG KPMDTVPLVMMAATKIGERQGQELYKEMQKRGWDVKESAVMAITANELDTARRRTTGSMD ALKAAGFPEKQIYQVPTKSNDI PGAFDAANSMLVQHPEVKHWLIVGMNDSTVLGGVRATE GQGFKAADI IGIGINGVDAVSELSKAQATGFYGSLLPSPDVHGYKSSEMLYNWVAKDVEP PKFTEVTDVVLITRDNFKEELEKKGLGGK

$>$ P38683

MRVLLFLLLSLFMLPAFSADNLLRWHDAQHFTVQASTPLKAKRAWKLCALYPSLKDSYWL SLNYGMQEAARRYGVDLKVLEAGGYSQLATQQAQ IDQCKQWGAEA I LLGSSTTSFPDLQK QVASLPVIELVNAIDAPQVKSRVGVPWFQMGYQPGRYLVQWAHGKPLNVLLMPGPDNAGG SKEMVEGFRAAIAGSPVRIVDIALGDNDIEIQRNLLQEMLERHPEIDVVAGTAIAAEAAM GEGRNLKTPLTVVSFYLSHQVYRGLKRGRVIMAASDQMVWQGELAVEQAIRQLQGQSVSD NVSPPILVLTPKNADREHIRRSLSPGGFRPVYFYQHTSAAKK

$>$ Q59452

MKLTKVALFSLGLFGFSSMALAHGDHMHNHDTKMDTMSKDMMSMEKIVVPVQQLDPQNGN KDVGTVEITESAYGLVFTPKLHDLAHGLHGFH IHEKPSCEPKEKDGKLVAGLGAGGHWDP KQTQKHGYPWSDDAHMGDLPALFVMHDGSATTPVLAPRLKKLAEVKGHSLMIHAGGDNHS DHPAPLGGGGPRMACGVIK

$>$ POAFM2

MRHSVLFATAFATLISTQTFAADLPGKGITVNPVQSTITEETFQTLLVSRALEKLGYTVN KPSEVDYNVGYTSLASGDATFTAVNWTPLHDNMYEAAGGDKKFYREGVFVNGAAQGYLID KKTADQYKITNIAQLKDPKIAKLFDTNGDGKADLTGCNPGWGCEGAINHQLAAYELTNTV THNQGNYAAMMADTISRYKEGKPVFYYTWTPYWVSNELKPGKDVVWLQVPFSALPGDKNA DTKLPNGANYGFPVSTMHIVANKAWAEKNPAAAKLFAIMQLPVADINAQNAIMHDGKASE GDIQGHVDGWIKAHQQQFDGWVNEALAAQK

$>$ P0A326

TDRP IMTTSAGAP I PDNQNSLTAGERGP I LMQDYQLIEKLSHQNRER I PERAVHAKGWGA YGTLTITGDISRYTKAKVLQPGAQTPMLARFSTVAGELGAADAERDVRGFALKFYTQEGN WDLVGNNTPVFFVRDPLKFPDF IHTQKRHPRTHLRSATAMWDFWSLSPESLHQVTILMSD RGLPTDVRHINGYGSHTYSFWNDAGERYWVKFHFKTMQGHKHWTNAEAEQVIGRTRESTQ EDLFSAIENGEFPKWKVQVQIMPELDADKTPYNPFDLTKVWPHADYPPIDIGVMELNRNP ENYFTEVENAAFSPSNIVPGIGFSPDKMLQARIFSYADAHRHRLGTHYES I PVNQPKCPV HHYHRDGQMNVYGGIKTGNPDAYYEPNSFNGPVEQPSAKEPPLCISGNADRYNHRIGNDD YSQPRALFNLFDAAQKQRLFSNIAAAMKGVPGFIVERQLGHFKLIHPEYEAGVRKALKDA HGYDANTIALNEKITAAE

$>$ P23847

MRISLKKSGMLKLGLSLVAMTVAASVQAKTLVYCSEGS PEGFNPQLFTSGTTYDASSVPL YNRLVEFKIGTTEVI PGLAEKWEVSEDGKTYTFHLRKGVKWHDNKEFKPTRELNADDVVF SFDRQKNAQNPYHKVSGGSYEYFEGMGLPELISEVKKVDDNTVQFVLTRPEAPFLADLAM DFAS ILSKEYADAMMKAGTPEKLDLNPIGTGPFQLQQYQKDSRIRYKAFDGYWGTKPQID TLVFS ITPDASVRYAKLQKNECQVMPYPNPADIARMKQDKS INLMEMPGLNVGYLSYNVQ KKPLDDVKVRQALTYAVNKDAI I KAVYQGAGVSAKNLI PPTMWGYNDDVQDYTYDPEKAK ALLKEAGLEKGFS IDLWAMPVQRPYNPNARRMAEMIQADWAKVGVQAKIVTYEWGEYLKR AKDGEHQTVMMGWTGDNGDPDNFFATLFSCAASEQGSNYSKWCYKPFEDLIQPARATDDH NKRVELYKQAQVVMHDQAPALI IAHSTVFEPVRKEVKGYVVDPLGKHHFENVS IE $>$ P4 2790

MKSSAAKQTVLCLNRYAVVALPLAIASFAAFGASPASTLWAPTDTKAFVTPAQVEARSAA PLLELAAGETAHIVVSLKLRDEAQLKQLAQAVNQPGNAQFGKFLKRRQFLSQFAPTEAQV QAVVAHLRKNGFVNIHVVPNRLLISADGSAGAVKAAFNTPLVRYQLNGKAGYANTAPAQV PQDLGEIVGSVLGLQNVTRAHPMLKVGERSAAKTLAAGTAKGHNPTEFPTIYDASSAPTA ANTTVGI ITIGGVSQTLQDLQQFTSANGLASVNTQTIQTGSSNGDYSDDQQGQGEWDLDS QS IVGSAGGAVQQLLFYMADQSASGNTGLTQAFNQAVSDNVAKVINVSLGWCEADANADG TLQAEDRIFATAAAQGQTFSVSSGDEGVYECNNRGYPDGSTYSVSWPASSPNVIAVGGTT LYTTSAGAYSNETVWNEGLDSNGKLWATGGGYSVYESKPSWQSVVSGTPGRRLLPDISFD AAQGTGALIYNYGQLQQIGGTSLASPIFVGLWARLQSANSNSLGFPAASFYSAISSTPSL VHDVKSGNNGYGGYGYNAGTGWDYPTGWGSLDIAKLSAYIRSNGFGH 
$>$ P42213

MKLFKS ILLIAACHAAQASATIDINADPNLTGAAPLTGILNGQKSDTQNMSGFDNTPPPA PPVVMSRMFGAQLFNGTSADSGATVGFNPDYILNPGDS IQVRLWGAFTFDGALQVDPKGN IFLPNVGPVKIAGVSNSQLNALVTSKVKEVYQSNVNVYASLLQAQPVKVYVTGFVRNPGL YGGVTSDSLLNYLIKAGGVDPERGSYVDIVVKRGNRVRSNVNLYDFLLNGKLGLSQFADG DTI IVGPRQHTFSVQGDVFNSYDFEFRESS I PVTEALSWARPKPGATHITIMRKQGLQKR SEYYPISSAPGRMLQNGDTLIVSTDRYAGTIQVRVEGAHSGEHAMVLPYGSTMRAVLEKV RPNSMSQMNAVQLYRPSVAQRQKEMLNLSLQKLEEASLSAQSSTKEEASLRMQEAQLISR FVAKARTVVPKGEVILNESNIDSVLLEDGDVINI PEKTSLVMVHGEVLFPNAVSWQKGMT TEDYIEKCGGLTQKSGNARI IVIRQNGARVNAEDVDSLKPGDEIMVLPKYESKNIEVTRG ISTILYQLAVGAKVILSL

$>$ POAEQ3

MKSVLKVSLAALTLAFAVSSHAADKKLVVATDTAFVPFEFKQGDKYVGFDVDLWAAIAKE LKLDYELKPMDFSGI I PALQTKNVDLALAGITITDERKKAIDFSDGYYKSGLLVMVKANN NDVKSVKDLDGKVVAVKSGTGSVDYAKANI KTKDLRQF PNIDNAYMELGTNRADAVLHDT PNILYFIKTAGNGQFKAVGDSLEAQQYGIAFPKGSDELRDKVNGALKTLRENGTYNEIYK KWFGTEPK

$>$ P18956

MIKPTFLRRVAIAALLSGSCFSAAAAPPAPPVSYGVEEDVFHPVRAKQGMVASVDATATQ VGVDILKEGGNAVDAAVAVGYALAVTHPQAGNLGGGGFMLIRSKNGNTTAIDFREMAPAK ATRDMFLDDQGNPDSKKSLTSHLASGTPGTVAGFSLALDKYGTMPLNKVVQPAFKLARDG FIVNDALADDLKTYGSEVLPNHENSKAIFWKEGEPLKKGDTLVQANLAKSLEMIAENGPD EFYKGTIAEQIAQEMQKNGGLITKEDLAAYKAVERTPISGDYRGYQVYSMPPPSSGGIHI VQILNILENFDMKKYGFGSADAMQIMAEAEKYAYADRSEYLGDPDFVKVPWQALTNKAYA KS IADQIDINKAKPSSEIRPGKLAPYESNQTTHYSVVDKDGNAVAVTYTLNTTFGTGIVA GESGILLNNQMDDFSAKPGVPNVYGLVGGDANAVGPNKRPLSSMSPTIVVKDGKTWLVTG SPGGSRI ITTVLQMVVNS IDYGLNVAEATNAPRFHHQWLPDELRVEKGFSPDTLKLLEAK GQKVALKEAMGSTQS IMVGPDGELYGASDPRSVDDLTAGY

$>$ P16700

MAVNLLKKNSLALVASLLLAGHVQATELLNSSYDVSRELFAALNPPFEQQWAKDNGGDKL TIKQSHAGSSKQALA ILQGLKADVVTYNQVTDVQILHDKGKL I PADWQSRLPNNSSPFYS TMGFLVRKGNPKNI HDWNDLVRSDVKLI F PNPKTSGNARYTYLAAWGAADKADGGDKGKT EQFMTQFLKNVEVFDTGGRGATTTFAERGLGDVLISFESEVNNIRKQYEAQGFEVVIPKT NILAEFPVAWVDKNVQANGTEKAAKAYLNWLYSPQAQTI ITDYYYRVNNPEVMDKLKDKF PQTELFRVEDKFGSWPEVMKTHFTSGGELDKLLAAGRN

$>Q 47459$

MKKILVSFVAIMAVASSAMAAETMNMHDQVNNAQAPAHQMQSSAEKSAVQGDSMTMMDMS SHDQAAMSHDMMQNGNSAAHQDMAEMHKKMMKSKPAASNETAKSFSEMNEHEKSAVVHEK ANNGQSSVIHQQQAEKHRSQITQN

$>$ P06875

MKNRNRMIVNCVTASLMYYWSLPALAEQSSSEIKIVRDEYGMPHIYANDTWHLFYGYGYV VAQDRLFQMEMARRSTQGTVAEVLGKDFVKFDKDIRRNYWPDAIRAQIAALSPEDMS ILQ GYADGMNAWIDKVNTNPETLLPKQFNTFGFTPKRWEPFDVAMIFVGTMANRFSDSTSEID NLALLTALKDKYGVSQGMAVFNQLKWLVNPSAPTTIAVQESNYPLKFNQQNSQTAALLPR YDLPAPMLDRPAKGADGALLALTAGKNRETIAAQFAQGGANGLAGYPTTSNMWVIGKSKA QDAKAIMVNGPQFGWYAPAYTYGIGLHGAGYDVTGNTPFAYPGLVFGHNGVISWGSTAGF GDDVDIFAERLSAEKPGYYLHNGKWVKMLSREETITVKNGQAETFTVWRTVHGNILQTDQ TTQTAYAKSRAWDGKEVASLLAWTHQMKAKNWQEWTQQAAKQALTINWYYADVNGNIGYV HTGAYPDRQSGHDPRLPVPGTGKWDWKGLLPFEMNPKVYNPQSGYIANWNNSPQKDYPAS DLFAFLWGGADRVTEIDRLLEQKPRLTADQAWDVIRQTSRQDLNLRLFLPTLQAATSGLT QSDPRRQLVETLTRWDGINLLNDDGKTWQQPGSAILNVWLTSMLKRTVVAAVPMPFDKWY SASGYETTQDGPTGSLNI SVGAKILYEAVQGDKSPI PQAVDLFAGKPQQEVVLAALEDTW ETLSKRYGNNVSNWKTPAMALTFRANNFFGVPQAAAEETRHQAEYQNRGTENDMIVFSPT TSDRPVLAWDVVAPGQSGF IAPDGTVDKHYEDQLKMYENFGRKSLWLTKQDVEAHKESQE VLHVQR

$>Q 3 \mathrm{~J} 2 \mathrm{P} 2$

MRPI PALALTFSLVAMPALAQDARQIERMIEGRHGLMTLMAHELGKLGGMAKEETPYDAE VAGKAASNLSALASVISPELFPKGSAVGEAEDSEALPAIWEKPDDFAQKISGMEEAAAKM QAAAGTDLASLQGAMRDLGAACGSCHETYR 
$>$ P26982

MKKTTLAMSALALSLGLALSPLSATAAETSSSAMTAQQMPSLAPMLEKVMPSVVS INVEG STTVNTPRMPRNFQQFFGDDSPFCQDGSPFQNSPFCQGGGNGGNGGQQQKFMALGSGVI I DAAKGYVVTNNHVVDNASVI KVQLSDGRKFDAKVVGKDPRSDIALIQIQNPKNLTAIKLA DSDALRVGDYTVAIGNPFGLGETVTSGIVSALGRSGLNVENYENFIQTDAAINRGNSGGA LVNLNGELIGINTA I LAPDGGNIGIGFA I PSNMVKNLTSQMVEYGQVKRGELGIMGTELN SELAKAMKVDAQRGAFVSQVMPNSSAAKAGIKAGDVITSLNGKPISSFAALRAQVGTMPV GSKISLGLLREGKAITVNLELQQSSQSQVDSSTIFSGIEGAEMSNKGQDKGVVVSSVKAN SPAAQ IGLKKGDVI I GANQQPVKNIAELRKILDSKPSVLALNIQRGDSS I YLLMQ $>$ P44984

MKLLKLTLISTALFSTAALAQAQQSVNVYSYDSFTSEWGAGPKVKQDFEKAHPQCAINFT PFESVGVLLNRVRLEGKKTKADIVLGLDNFFLEQAEKTGIFAPNNVDLTQLDLPTKWANK TFLPFDFGNYAFVYDKTKLQNPPKSLKELVERQDLSVIYQDPRTSSVGRGLLVWMNAVYP ADKIQSAWKELDKHTVTVGKGWSDTYGAFLKGEADLVLSYSTSPLYHQLFEKKDNYAATD FAEGHITQVELAARVANHPNQCADDFMAFLISPTAQKHIVTANIMLPVIQGEIEPHFDAL KVQQKTQTS INPMVNTEQLKNWISTWQTTLTK

$>Q 9 L 7 P 2$

MQTPKLIRPTLLSMAILSSMAWATGASAALVPPKGYDAPIEKMKTGDHNFSCEAI PKPYT DKLVFRSKYEGSDKARATLNAVSEEAFRDATKDITTLERGVSKVVMQYMRDGRPEQLDCA LNMMTTWAKADALESREFNHTGKSMRKWALGSMSSAYLRLKFSESHPLANRQQDAKI IET WFSKLADQVVSDWSNLPLEKINNHSYWAAWSVMATAVATNRQDLFDWAVKEYKVAANQVD KDGFLPNEMKRRQRALSYHNYALPPLAMIASFAQANGVDLRPENNGALKRLGDRVLAGVK DPS IFAEHNGEKQDMTDLKKDPKFAWLEPYCSLYTCSPDVLEEKHEKQPFKTFRLGGDLT KVYDPTHEKGDKGDNDGS

$>Q 51705$

MESKQEKGLSRRALLGATAGGAAVAGAFGGRLALGPAALGLGTAGVATVAGSGAALAASG DGSVAPGQLDDYYGFWSSGQSGEMRILGI PSMRELMRVPVFNRCSATGWGQTNESVRIHE RTMSERTKKFLAANGKRIHDNGDLHHVHMSFTEGKYDGRFLFMNDKANTRVARVRCDVMK CDAILEI PNAKGIHGLRPQKWPRSNYVFCNGEDETPLVNDGTNMEDVANYVNVFTAVDAD KWEVAWQVLVSGNLDNCDADYEGKWAFSTSYNSEKGMTLPEMTAAEMDHIVVFNIAEIEK AIAAGDYQELNGVKVVDGRKEASSLFTRY I PIANNPHGCNMAPDKKHLCVAGKLSPTATV LDVTRFDAVFYENADPRSAVVAEPELGLGPLHTAFDGRGNAYTSLFLDSQVVKWNIEDAI RAYAGEKVDP I KDKLDVHYQPGHLKTVMGETLDATNDWLVCLSKFSKDRFLNVGPLKPEN DQLIDISGDKMVLVHDGPTFAEPHDA IAVHPS ILSDI KSVWDRNDPMWAETRAQAEADGV DIDNWTEEVIRDGNKVRVYMSSVAPSFSIESFTVKEGDEVTVIVTNLDEIDDLTHGFTMG NYGVAMEIGPQMTSSVTFVAANPGVYWYYCQWFCHALHMEMRGRMLVEPK

$>$ P29898

MKRILTLTVAALALGTPALAYDGTNCKAPGNCWEPKPDYPAKVEGSKYDPQHDPAELSKQ GESLAVMDARNEWRVWNMKKTGKFEYDVKKIDGYDETKAP

$>$ P07102

MKAILIPFLSLLIPLTPQSAFAQSEPELKLESVVIVSRHGVRAPTKATQLMQDVTPDAWP TWPVKLGWLTPRGGELIAYLGHYQRQRLVADGLLAKKGCPQSGQVAI IADVDERTRKTGE AFAAGLAPDCAITVHTQADTSSPDPLFNPLKTGVCQLDNANVTDA I LSRAGGS IADFTGH RQTAFRELERVLNFPQSNLCLKREKQDESCSLTQALPSELKVSADNVSLTGAVSLASMLT EIFLLQQAQGMPEPGWGRITDSHQWNTLLSLHNAQFYLLQRTPEVARSRATPLLDLIKTA LTPHPPQKQAYGVTLPTSVLFIAGHDTNLANLGGALELNWTLPGQPDNTPPGGELVFERW RRLSDNSQWIQVSLVFQTLQQMRDKTPLSLNTPPGEVKLTLAGCEERNAQGMCSLAGFTQ IVNEARIPACSL

$>$ POABE7

MRKSLLAILAVSSLVFSSASFAADLEDNMETLNDNLKVIEKADNAAQVKDALTKMRAAAL DAQKATPPKLEDKSPDSPEMKDFRHGFDILVGQIDDALKLANEGKVKEAQAAAEQLKTTR NAYHQKYR

$>$ P19843

MFKAQATFSRYSAAVSLLLLFSGAAQAAPQSITTLPLQPDGENRWRLPAGEYQGQFTIEQ PMQLRCEPGAVIQSQGQGSSLLISAPDVLVEGCTLYEWGSDLTAMDSAVF ILPAAERAQI SNNRMRGPGFGVFVDGTRDVQVI GNEIDGDAGVRSQDRGNGIHLFAVSGARVLHNHVRNA RDGIYIDTSNGNHLEGNVIEDVRYGVHYMFANENSLIDNVTRRTRTGYALMQSRKLTVTG NRSEQDQNYGILMNY ITYSTITGNFVSDVQRGDTGGDSMI SGGEGKALF I YNSLFNTIEN NHFEKSSLGIHLTAGSEDNRISGNAFVGNQQQVKYVASRTQEWSVDGRGNYWSDYLGWDR 
NNDGLGDIAYEPNDNVDRLLWLYPQVRLLMNSPS IEVLRWVQRAFPVIKSPGVQDSHPLM KLPTEKLLTEKQEPTS

$>$ P0A 855

MKQALRVAFGFLILWASVLHAEVRIVIDSGVDSGRPIGVVPFQWAGPGAAPEDIGGIVAA DLRNSGKFNPLDRARLPQQPGSAQEVQPAAWSALGIDAVVVGQVTPNPDGSYNVAYQLVD TGGAPGTVLAQNSYKVNKQWLRYAGHTASDEVFEKLTGI KGAFRTRIAYVVQTNGGQFPY ELRVSDYDGYNQFVVHRSPQPLMSPAWS PDGSKLAYVTFESGRSALVIQTLANGAVRQVA SFPRHNGAPAFSPDGSKLAFALSKTGSLNLYVMDLASGQIRQVTDGRSNNTEPTWFPDSQ NLAFTSDQAGRPQVYKVNINGGAPQRITWEGSQNQDADVSSDGKFMVMVSSNGGQQHIAK QDLATGGVQVLSSTFLDETPSLAPNGTMVIYSSSQGMGSVLNLVSTDGRFKARLPATDGQ VKFPAWSPYL

$>Q 07982$

MTNKISSSDNLSNAVSATDDNASRTPNLTRRALVGGGVGLAAAGALASGLQAATLPAGAS QVPTTPAGRPMPYAIRPMPEDRRFGYAIVGLGKYALNQILPGFAGCQHSRIEALVSGNAE KAKIVAAEYGVDPRKIYDYSNFDKIAKDPKIDAVYI I LPNSLHAEFAIRAFKAGKHVMCE KPMATSVADCQRMIDAAKAANKKLMIGYRCHYDPMNRAAVKLIRENQLGKLGMVTTDNSD VMDQNDPAQQWRLRRELAGGGSLMDIGIYGLNGTRYLLGEEPIEVRAYTYSDPNDERFVE VEDRI IWQMRFRSGALSHGASSYSTTTTSRFSVQGDKAVLLMDPATGYYQNLISVQTPGH ANQSMMPQF IMPANNQF SAQLDHLAEAVINNKPVRS PGEEGMQDVRLIQA IYEAARTGRP VNTDWGYVRQGGY

$>$ P18035

MSLTKSLLFTLLLSAAAVQASTRDEIERLWNPQGMATQPAQPAAGTSARTAKPAPRWFRL SNGRQVNLADWKVVLFMQGHCPYCHQFDPVLKQLAQQYGFSVFSYTLDGQGDTAFPEALP VPPDVMQTFFPNI PVATPTTFLVNVNTLEALPLLQGATDAAGFMARVDTVLQMYGGKKGA $\mathrm{K}$

$>$ P18471

MKRRLWLLMLFLFAGHVPAASADSACEGRFVNPITDICWSCIFPLSLGS I KVSQGKVPDT ANPSMPIQICPAPPPLFRRIGLAIGYWEPMALTDVTRSPGCMVNLGFSLPAFGKTAQGTA KKDEKQVNGAFYHVHWYKYPLTYWLNI ITSLGCLEGGDMDIAYLSEIDPTWTDSSLTTIL NPEAVIFANP IAQGACAADA IASAFNMPLDVLFWCAGSQGSMYPFNGWVSNESSPLQSSL LVSERMAFKLHRQGMIMETIGKNNAVCNEYPSP I LPKERWRYQMVNMYPDSGQCHPFGRS VTRWETGKNP PNTKKNFGYLMWRKRNCVFL

$>$ POAAA9

MKRNTKIALVMMALSAMAMGSTSAFAHGGHGMWQQNAAPLTSEQQTAWQKIHNDFYAQSS ALQQQLVTKRYEYNALLAANPPDSSKINAVAKEMENLRQSLDELRVKRDIAMAEAGIPRG AGMGMGYGGCGGGGHMGMGHW

$>$ P12374

MESRTSRRTFVKGLAAAGVLGGLGLWRSPSWAASGSPALSVLSGTEFDLSIGEMPVNITG RRRTAMA INGGLPGPLLRWKEGDTVTLRVRNRLDAATS IHWHGI I LPPNMDGVPGLSFAG IEPGGVYVYQFKVQQNGTYWYHSHSGFQEQVGVYGPLVIEAKEPEPFKYDSEHVVMLTDW TDEDPVSLMRTLKKQSDYYNFHKRTVGDFVNDVADKGWAATVADRKMWAEMKMNPTDLAD VSGATYTYLLNGQAPNMNWTGLFRPGEKLRLRF INGSAMTYFDIRI PGLKMTVVASDGQF VNPVEVDELRIAVAETFDVIVEPTAEAYTVFAQSMDRTGYARGTLAVREGLVAQVPPLDP RPLVTMDDMGMGGMDHGSMDGMSGMDSGADDGMQTMSSMGGDSMPAMDHSKMSTMQGMDH GAMSGMDHGAMGGMVMQSHPASENDNPLVDMQAMSPTAKLNDPGLGLRNNGRKVLTYADL KSTFEDPDGREPSRTIELHLTGHMEKFAWSFDGIKFADAQPLILKYGERVRIVLVNDTMM THP IHLHGMWSDLEDEDGNFRVRKHTIDMPPGSKRSYRVTADALGRWAYHCHLLYHMEMG MFREVRVEE

$>$ P10488

MYDRWFSQQELQVLPFADEDEQRNQTWLELVGEAQQLMANAARQMSRGRLRWQPAGWSSW SRIHRHAEFLTRLNEMHAAEPQMREQTGVTPEMIDF ITRAFAESKLAIWARYLNAEELAF TRQHYFDRLMEWPALVADLHRACREKRDRPPRKVSSWRSAAWRCSSLTRVKMRRRSRISL CHAQVPALMKGTWMTSEVLSWLQQAIGVMMRQAQGPRE

$>$ P12376

MLLNRTSFVTLFAAGMLVSALAQAHPKLVSSTPAEGSEGAAPAKIELHFSENLVTQFSGA KLVMTAMPGMEHS PMAVKAAVSGGGDPKTMVITPAS PLTAGTYKVDWRAVSSDTHPITGS VTFKVK

$>$ POAEE1

MRNLVKYVGIGLLVMGLAACDDKDTNATAQGSVAESNATGNPVNLLDGKLSFSLPADMTD 
QSGKLGTQANNMHVWSDATGQKAVIVIMGDDPKEDLAVLAKRLEDQQRSRDPQLQVVTNK AIELKGHKMQQLDS I ISAKGQTAYSSVI LGNVGNQLLTMQITLPADDQQKAQTTAENI IN TLVIQ

$>$ Q9X4Y1

MKTHRLNMTASLLIGISAFAVQAFASEPTVVPEQPPFPAQGKITYVSRDSILEFKALREY REPEWVTEKFVKAGKLPPVAERLPKEPMVFKAGNMPDGMGVYGDVMRHVIGGRPEGWNYS AGQTQGWGGIDIGMFECLTRTAPLFQVEADDMEPLPNLAKSWDWSEDGRKLTMHLIEGAK WSDGDPFDADDVMFYWEDNVLDSSVSPLNGATPETFGEGTTLKKIDQYTVEWTFKEAFPR QHLFAMAYGTFCPGPSHILKTKHPKYAGTTYNEYKNGFPAEYMNLPVMGAWVPVAYRPDD I IVLRRNPYYWKVDEAGNQLPYLNELHYKLSTWADRDVQA IAGSGDI SNLEQPENFVESL KRAANESAPARLAFGPRVIGYNMHMNFSGNGWGDPDERAKAVRELNRNLDFRKAVTMAVD RKKLGEALVKGPFTAIYPGGLSSGTSFYDRNSTIYYPHDLEGAKVLLEKVGLKDTDGNGF VNFPAGKLGGRDVEIVLLVNSDYSTDRNLAEGMVGQMEKLGLRVVLNALDGKQRDAANYA GRFDWMIHRNTAEFASVVQNTPQLAPTGPRTSWHHRAPEGGEVDVMPHEQELVDIVNKFI ASNDNDERTELMKQYQKVATTNVDTVGLTEYPGAL I INKRF SN I PPGAP I FMFNWAEDT I IRERVFVAADKQGDYELYPEQLPGKPGESGPIN $>Q 50925$

MRIGEWMRGLLLCAGLMMCGVVHADISTVPDETYDALKLDRGKATPKETYEALVKRYKDP AHGAGKGTMGDYWEPIAIS IYMDPNTFYKPPVSPKEVAERKDCVECHSDETPVWVRAWKR STHANLDKIRNLKSDDPLYYKKGKLEEVENNLRSMGKLGEKETLKEVGCIDCHVDVNKKD KADHTKDIRMPTADTCGTCHLREFAERESERDTMVWPNGQWPAGRP SHALDYTANIETTV WAAMPQREVAEGCTMCHTNQNKCDNCHTRHEFSAAESRKPEACATCHSGVDHNNWEAYTM SKHGKLAEMNRDKWNWEVRLKDAFSKGGQNAPTCAACHMEYEGEYTHNITRKTRWANYPF VPGIAENITSDWSEARLDSWVLTCTQCHSERFARSYLDLMDKGTLEGLAKYQEANAIVHK MYEDGTLTGQKTNRPNPPEPEKPGFGIFTQLFWSKGNNPASLELKVLEMAENNLAKMHVG LAHVNPGGWTYTEGWGPMNRAYVEIQDEYTKMQELSALQARVNKLEGKQTSLLDLKGTGE KISLGGLGGGMLLAGALALIGWRKRKQTRA

$>$ P2 6648

MSLSRRQFIQASGIALCAGAVPLKASAAGQQQPLPVPPLLESRRGQPLFMTVQRAHWSFT PGTRASVWGINGRYLGPTIRVWKGDDVKLIYSNRLTENVSMTVAGLQVPGPLMGGPARMM SPNADWAPVLP IRQNAATLWYHANTPNRTAQQVYNGLAGMWLVEDEVSKSLP I PNHYGVD DFPVI IQDKRLDNFGTPEYNEPGSGGFVGDTLLVNGVQSPYVEVSRGWVRLRLLNASNSR RYQLQMNDGRPLHVISGDQGFLPAPVSVKQLSLAPGERREILVDMSNGDEVS ITCGEAAS IVDRIRGFFEPSSILVSTLVLTLRPTGLLPLVTDSLPMRLLPTEIMAGSP IRSRDISLGD DPGINGQLWDVNR IDVTAQQGTWERWTVRADEPQAFHIEGVMFQIRNVNGAMPFPEDRGW KDTVWVDGQVELLVYFGQPSWAHF PFYFNSQTLEMADRGS IGQLLVNPVP

$>$ POADA1

MMNFNNVFRWHLPFLFLVLLTFRAAAADTLLILGDSLSAGYRMSASAAWPALLNDKWQSK TSVVNAS I SGDTSQQGLARLPALLKQHQPRWVLVELGGNDGLRGFQPQQTEQTLRQILQD VKAANAEPLLMQIRLPANYGRRYNEAFSA IYPKLAKEFDVPLLPFFMEEVYLKPQWMQDD GIHPNRDAQPFIADWMAKQLQPLVNHDS

$>Q 01537$

MSEQFRLTRRSMLAGAAVAGALAPVVTSVAHAEGGGIKTNSAATAANIATLERVKVELVK PPFVHAHTQKAEGEPKVVEFKMTIQEKKIVVDDKGTEVHAMTFDGSVPGPMMIVHQDDYV ELTLVNPDTNELQHNIDFHSATGALGGGALTVVNPGDTAVLRFKATKAGVFVYHCAPAGM VPWHVTSGMNGA IMVLPRDGLKDHKGHELVYDKVYYVGEQDFYVPKDENGKFKKYESAGE AYPDVLEAMKTLTPTHVVFNGAVGALTGDNALQAKVGDRVLILHSQANRDTRPHLIGGHG DYVWATGKFANP PELDQETWF I PGGAAGAAYYTFQQPGIYAYVNHNLIEAFELGAAGHFK VTGDWNDDLMTAVVSPTSG

$>$ P2 4037

MKKTLMASAVGAVIAFGTHGAMAAAPADWSSVAATDVTLFYPGVSPVEWITKGTEHGGAR ALKKGETCAGCHSEEASDMGEKMASGKKLEPSPIAGKAPF INAKVQAANDGENLYLRFTW KQPAASGAAPMDADNPVKIAYMLEGGSKVELAEAGGCWGSCHGDARTMPGAADTKTKYVK DGSLANGVYYDLNQWRSGENKAFDGYVATERVMEGGQALVDAQGKLDGDTWTVVFTRKFA GGEGDVTLAPGNLYNFGFAIHDDSATGRYHHVSLGYSLGIDAQGDITAAKQ

$>$ P19147

MQPAVSLIAGAVLSALLCSSAIAAETSANADGLTDRAARGNLVEPGGARRLAGDQTTALK ASLSDKTAKNVILLIGDGMGDSEITAARNYAEGAGGYFKGIDALPLTGQYTHYSLDRKTH KPDYVTDSAASATAWATGVKTYNGALGVDVNGKDQPTLLEIAKAAGKATGNVSTAELQDA 
TPAALVSHVISRKCYGPEETSEKCAANALENGGRGSITEQLLKTRADVTLGGGAKSFNQL AKSGEWQGKSLKDQAAAQGYQWVSNADELQAVTLANQQKPLLGLFADGNMPVRWLGPKAS YHGNLDKPAVTCENNPARTAATPTLAAMTEKAIALLKDNPNGFFLQVEGAS IDKQDHAAN PCGQIGETVDLDEAVQKALAFARADGNTLVIVTADHAHASQIVSADAKAPGLTQKLTTKD GAPMTLSYGNSEEESQGHTGTQLRVAAYGPHAANVVGLTDQTDLFFTMRDAMGIK

$>$ P39325

MWKRLLIVSAVSAAMSSMALAAPLTVGFSQVGSESGWRAAETNVAKSEAEKRGITLKIAD GQQKQENQIKAVRSFVAQGVDA I FIAPVVATGWEPVLKEAKDAEI PVFLLDRS IDVKDKS LYMTTVTADNILEGKLIGDWLVKEVNGKPCNVVELQGTVGASVAIDRKKGFAEAIKNAPN IKIIRSQSGDFTRSKGKEVMESFIKAENNGKNICMVYAHNDDMVIGAIQAIKEAGLKPGK DILTGS IDGVPDIYKAMMDGEANASVELTPNMAGPAFDALEKYKKDGTMPEKLTLTKSTL YLPDTAKEELEKKKNMGY

$>$ Q 8VUS8

MNALVGCTTSFDPGWEVDAFGAVSNLCQPMEADLYGCADPCWWPAQVADTLNTYPNWSAG ADDVMQDWRKLQSVFPETKGSS

$>068897$

MKSALKTFVPGALALLLLFPVAAQAKEVETKTKLANVVILATGGTIAGAGASAANSATYQ AAKVGIEQLIAGVPELSQIANVRGEQVMQIASES INNENLLQLGRRVAELADS KDVDGIV ITHGTDTLEETAYFLNLVEKTDKPI IVVGSMRPGTAMSADGMLNLYNAVAVAGSKDARGK GVLVTMNDEIQSGRDVSKMINI KTEAFKSPWGPLGMVVEGKSYWFRLPAKRHTMDSEFDI KTIKSLPDVEIAYGYGNVSDTAVKALAQAGAKAI I HAGTGNGSVSSKVVPALQELRKQGV QI IRSSHVNAGGFVLRNAEQPDDKYDWVVAHDLNPQKARILAMVALTKTQDSKELQRMFW $\mathrm{EY}$

$>$ P33225

MNNNDLFQASRRRFLAQLGGLTVAGMLGPSLLTPRRATAAQAATDAVISKEGILTGSHWG AIRATVKDGRFVAAKPFELDKYPSKMIAGLPDHVHNAARIRYPMVRVDWLRKRHLSDTSQ RGDNRFVRVSWDEALDMFYEELERVQKTHGPSALLTASGWQSTGMFHNASGMLAKAIALH GNSVGTGGDYSTGAAQVILPRVVGSMEVYEQQTSWPLVLQNSKTIVLWGSDLLKNQQANW WCPDHDVYEYYAQLKAKVAAGEIEVISIDPVVTSTHEYLGREHVKHIAVNPQTDVPLQLA LAHTLYSENLYDKNFLANYCVGFEQFLPYLLGEKDGQPKDAAWAEKLTGIDAETIRGLAR QMAANRTQI IAGWCVQRMQHGEQWAWMI VVLAAMLGQIGLPGGGFGFGWHYNGAGTPGRK GVILSGFSGSTSI PPVHDNSDYKGYSSTIPIARF IDAI LEPGKVINWNGKSVKLPPLKMC IFAGTNPFHRHQQINR I IEGLRKLETVIAIDNQWTSTCRFADIVLPATTQFERNDLDQYG NHSNRGI IAMKQVVPPQFEARNDFDI FRELCRRFNREEAFTEGLDEMGWLKRIWQEGVQQ GKGRGVHLPAFDDFWNNKEYVEFDHPQMFVRHQAFREDPDLEPLGTPSGLIEIYSKTIAD MNYDDCQGHPMWFEKIERSHGGPGSQKYPLHLQSVHPDFRLHSQLCESETLRQQYTVAGK EPVF INPQDASARGIRNGDVVRVFNARGQVLAGAVVSDRYAPGVARIHEGAWYDPDKGGE PGALCKYGNPNVLTIDIGTSQLAQATSAHTTLVEIEKYNGTVEQVTAFNGPVEMVAQCEY VPASQVKS

$>$ P39186

MKPSRSWASLLAVCAVLLAALAMQA IFFPAPARAQGLVDAMRGPTAIANEPRAPLLYPTE NKDIRRTRNYTMQPPTI PHKIDGYQLDKDFNRCMFCHARTRTEETQAI PVSITHYMDRDN NVLADVSPRRYFCTQCHVPQADTKPLIGNNFVDVDTILKRRPGAKGAAK

$>$ P23827

MKTILPAVLFAAFATTSAWAAESVQPLEKIAPYPQAEKGMKRQVIQLTPQEDESTLKVEL LIGQTLEVDCNLHRLGGKLENKTLEGWGYDYYVFDKVSSPVSTMMACPDGKKEKKFVTAY LGDAGMLRYNSKLPIVVYTPDNVDVKYRVWKAEEKIDNAVVR

$>$ POAE 85

MRIVTAAVMASTLAVSSLSHAAEVGSGDNWHPGEELTQRSTQSHMFDGISLTEHQRQQMR DLMQQARHEQPPVNVSELETMHRLVTAENFDENAVRAQAEKMANEQIARQVEMAKVRNQM YRLLTPEQQAVLNEKHQQRMEQLRDVTQWQKSSSLKLLSSSNSRSQ

$>$ P00099

MKPYALLSLLATGTLLAQGAWAEDPEVLFKNKGCVACHAIDTKMVGPAYKDVAAKFAGQA GAEAELAQRI KNGSQGVWGPI PMP PNAVSDDEAQTLAKWV

$>$ P2 7175

MSTTSRPGLWALITAAVFALCGAILTVGGAWVAAIGGPLYYVILGLALLATAFLSFRRNP AALYLFAVVVFGTVIWELTVVGLDIWALI PRSDIVI I LGIWLLLPFVSRQIGGTRTTVLP LAGAVGVAVLALFASLFTDPHDISGDLPTQIANASPADPDNVPASEWHAYGRTQAGDRWS PLNQINASNVSNLKVAWHIHTKDMMNSNDPGEATNEATP IEFNNTLYMCSLHQKLFAVDG 
ATGNVKWVYDPKLQINPGFQHLTCRGVSFHETPANATDSDGNPAPTDCAKRI ILPVNDGR LVEVDADTGKTCSGFGNNGEIDLRVPNQPYTTPGQYEPTSP PVITDKLI IANSAITDNGS VKQASGATQAFDVYTGKRVWVFDASNPDPNQLPDDSHPVFHPNSPNSWIVSSYDRNLNLV YIPMGVGTPDQWGGDRTKDSERFAPGIVALNADTGKLAWFYQTVHHDLWDMDVPSQPSLV DVTQKDGTLVPAIYAPTKTGDIFVLDRRTGKEIVPAPETPVPQGAAPGDHTSPTQPMSQL TLRPKNPLNDSDIWGGTIFDQMFCS IYFHTLRYEGPFTPPSLKGSLIFPGDLGMFEWGGL AVDPQRQVAFANPISLPFVSQLVPRGPGNPLWPEKDAKGTGGETGLQHNYGI PYAVNLHP FLDPVLLPFGI KMPCRTPPWGYVAGIDLKTNKVVWQHRNGTLRDSMYGSSLPI PLPPIKI GVPSLGGPLSTAGNLGFLTASMDYYIRAYNLTTGKVLWQDRLPAGAQATPITYAINGKQY IVTYAGGHNSFPTRMGDDI IAYALPDQK

$>$ P25718

MKLAACFLTLLPGFAVAASWTSPGFPAFSEQGTGTFVSHAQLPKGTRPLTLNFDQQCWQP ADA I KLNQMLSLQPCSNTPPQWRLFRDGEYTLQIDTRSGTPTLMIS IQNAAEPVASLVRE CPKWDGLPLTVDVSATFPEGAAVRDYYSQQIAIVKNGQ IMLQPAATSNGLLLLERAETDT SAPFDWHNATVYFVLTDRFENGDPSNDQSYGRHKDGMAE IGTFHGGDLRGLTNKLDYLQQ LGVNALWISAPFEQIHGWVGGGTKGDFPHYAYHGYYTQDWTNLDANMGNEADLRTLVDSA HQRGIRILFDVVMNHTGYATLADMQEYQFGALYLSGDEVKKSLGERWSDWKPAAGQTWHS FNDY INFSDKTGWDKWWGKNWIRTDIGDYDNPGFDDLTMSLAFLPDIKTESTTASGLPVF YKNKMDTHAKAIDGYTPRDYLTHWLSQWVRDYGIDGFRVDTAKHVELPAWQQLKTEASAA LREWKKANPDKALDDKPFWMTGEAWGHGVMQSDYYRHGFDAMINFDYQEQAAKAVDCLAQ MDTTWQQMAEKLQGFNVLSYLSSHDTRLFREGGDKAAELLLLAPGAVQIFYGDESSRPFG PTGSDPLQGTRSDMNWQDVSGKSAASVAHWQKISQFRARHPAIGAGKQTTLLLKQGYGFV REHGDDKVLVVWAGQQ 